\title{
ON THE INTERACTIONS BETWEEN
}

CLOUDS, radiation, tURBULENCE

\section{ANDVEGETATION}

IN THE ATMOSPHERIC BOUNDARY LAYER 


\section{On the interactions between clouds, radiation, turbulence and vegetation in the atmospheric boundary layer}




\section{Thesis committee}

\section{Promotor:}

Prof. Dr J. Vilà-Guerau de Arellano

Professor of Meteorology

Wageningen University and Research

\section{Other members:}

Prof. Dr N.P.R Anten, Wageningen University and Research Prof. Dr H.J.J. Jonker, Delft University of Technology Dr A.A. Nuijens, Delft University of Technology

Prof. Dr A. Verhoef, University of Reading, United Kingdom.

This research was conducted under the auspices of the Socio-Economic and Natural Sciences of the Environment (SENSE) Graduate School. 


\title{
On the interactions between clouds, radiation, turbulence and vegetation in the atmospheric boundary layer
}

\author{
Xabier Pedruzo Bagazgoitia
}

Thesis

submitted in fulfilment of the requirements for the degree of doctor at Wageningen University by the authority of the Rector Magnificus

Prof. Dr A.P.J. Mol, in the presence of the Thesis Committee appointed by the Academic Board to be defended in public on Friday 6 December 2019 at 11 a.m. in the Aula. 
Xabier Pedruzo Bagazgoitia

On the interactions between clouds, radiation, turbulence and vegetation in the atmospheric boundary layer.

200 pages.

PhD thesis, Wageningen University, Wageningen, NL (2019)

With references, with summary in English

DOI https://doi.org/10.18174/502754

ISBN 978-94-6395-160-9 
"You can know the name of a bird in all the languages of the world, but when you're finished, you'll know absolutely nothing whatever about the bird. So let's look at the bird and see what it's doing: that's what counts."

Richard Feynman 



\section{Contents}

Page

Contents vii

1 General Introduction 1

2 Shallow cumulus representation and its interaction with radiation and surface at the convection gray zone

3 Direct and diffuse radiation in the shallow cumulus-vegetation system: enhanced and decreased evapotranspiration regimes

4 The diurnal stratocumulus-to-cumulus transition over land

5 Emergent features on cloud convection by coupling 3D radiation and surface on Large Eddy Simulations

6 General Conclusions and Outlook

Summary/Resumen/Laburpena

Supplementary material

References

Acknowledgements

About the author and Publications

Graduate school certificate 



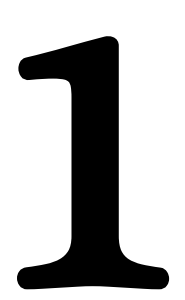

General Introduction 


\subsection{Motivation}

Vegetation and low clouds, studied in this thesis, are tightly coupled to the dynamics of the atmospheric boundary layer. This layer is defined as the lower part of the atmosphere where the conditions from the free troposphere, about the lowest $16 \mathrm{~km}$ of the atmosphere, connect with the conditions at the surface. As such, the boundary layer comprises the atmospheric region in which all the inhabitants of the Earth live in. Whether it is warm, cold, dry, moist, misty, clear, cloudy or sunny is conditioned by the continuously changing processes in the boundary layer. Within the boundary layer over land, the vegetation at the surface regulates what and in what quantities is transferred from the surface to the atmosphere, and vice versa. In turn, low clouds or boundary-layer clouds, i.e. those in which surface conditions are necessary for its appearance, affect the boundary layer through several processes. In particular, these clouds influence both the dynamics of the boundary layer and the radiation traveling through it, eventually impacting the Earth's surface. Thus, understanding how low clouds, vegetation and boundary layer interact requires an integrated interpretation, where a unified approach to the processes studied is critical. In fact, the motivations for our interdisciplinary study belong to different disciplines nowadays, as we will show next.

Firstly, and in the current context of a climate change, clouds and vegetation are two of the main actors in our climate. Clouds consist of the random but coherent collection of thousands of small and large drops. Due to the dependence of clouds on very small scale processes, they are currently one of the largest uncertainties in climate projections (Boucher et al., 2013). Plants, absorbing water from the soil and emitting part of it to the atmosphere through photosynthesis, are part of the global water cycle while they also affect local weather conditions. Furthermore, clouds and vegetation play an active role in the climate itself as they modify the temperature and moisture present at the surface and in the atmosphere. Under certain conditions, these modifications to the water cycle are key in the development of extreme weather events such as deep convection and thunderstorms. Other extreme events, e.g. heatwaves and droughts, are expected to occur more often and are particularly sensitive to the vegetation state and the available water at the surface (Boucher et al., 2013; Miralles et al., 2014). In turn, the $\mathrm{CO}_{2}$ exchanged between vegetation and atmosphere plays a very relevant role in the carbon cycle of the earth. In fact, the amount of $\mathrm{CO}_{2}$ absorbed by vegetation worldwide, known as the $\mathrm{CO}_{2}$ terrestrial sink, is largely uncertain and shows very large interannual variability (Keenan et al., 2012; Boucher et al., 2013). Thus, understanding how clouds and vegetation interact on the small scales increases the knowledge on the coupling between the global water and carbon cycles, and enables us to better interpret their evolution in a changing climate.

Secondly, human-induced perturbations on land use are happening worldwide. In the majority of the cases, this means the deforestation of vegetated areas for its use as agri- 
cultural fields, urban or industrial purposes. In other cases, land use is modified for the plain use of the resources themselves, such as deforestation for timber production. With such changes happening over increasingly larger areas, one could wonder whether such a modification at the surface has the potential to impact local weather. Furthermore, the potential of weather to feed back on surface conditions, for instance through precipitation, suggests to study the bi-directional effects of land use and atmosphere. Thus, a better knowledge on the cloud-surface relation appears to be very timely and necessary to assess how man-made surface changes modify the atmosphere, and vice versa.

Thirdly, in the last years more and more of the energy we employ comes from renewable sources. In particular, solar and wind energy are becoming increasingly common and popular because of their non-pollutant character, worldwide availability and lowering production costs. Gaining knowledge on the local relation between clouds and surface is key in promoting the growth and improvement of this field: finding the best location and conditions for energy farms, adequately forecasting energy generation peaks and drops or providing the extreme conditions that the solar panels or wind mills will undergo.

Inspired by these motivation elements, our work aims to integrate the multiple scientific fields needed to understand the interactions between vegetation, clouds, and atmosphere. The behavior of vegetation is a subject of study on itself, with biology being the main field aiming to understand and describe it. In turn, clouds, being part of the weather, belong to the meteorology or atmospheric science; but also to the field of hydrology, as clouds manifest the transport of water and determine the precipitation and, thus, the available water at the surface. There is currently plenty of knowledge with very detailed theories on the basis of each individual discipline. However, the interactions among them and their potential to affect each other's development have received less attention. For that purpose we combine knowledge and methodologies from the fields of biology, meteorology and hydrology to describe the processes in the land-atmosphere-cloud system in a coupled and cross-disciplinary way. We do so by considering their explicit effects, i.e. based on first principles, at the small scale were they take place.

The aim of this thesis is to understand, describe, and quantify the interactions, taking place along meters and within seconds, between the vegetated surface and the explicitly resolved atmospheric boundary layer topped by shallow cumulus, stratocumulus clouds and cloudless conditions, and the transitions among them.

The remaining part of the introduction is organized as follows: the phenomena and processes investigated in this thesis are introduced in Section 1.2. There, we describe the principles of clear and cloudy boundary layers, the active vegetation at the surface and the role of radiation, as well as the main modeling tools we employ to study such phenomena. Afterwards in Section 1.3 we present a summary of past research in the topic concerning both observations as well as modeling, with emphasis on the studies using Large Eddy 
Simulation. In Section 1.4 we provide the outline of this thesis and we briefly describe the content of each chapter.

\subsection{The atmospheric boundary layer: the turbulent scene where the dynamics of surface, radiation and clouds meet}

\subsubsection{The clear and turbulent boundary layer}

The boundary layer is the interface that lays between the surface of the earth and the free troposphere (Stull, 1988; Garratt, 1992). Above the boundary layer, thermodynamic conditions are independent of the surface, its roughness and its fluxes. The boundary layer and its properties, however, vary depending on the surface features and the time of the day. In our case, we study the boundary layer over land and during the daytime, usually called the convective boundary layer (CBL). During daytime, the CBL is the buffer between the quasi-laminar flow of the free troposphere and the rigid surface and is, consequently, of turbulent nature. This means that properties such as temperature, moisture or wind fluctuate along meters and within seconds due to the chaotic movements of the flow. The boundary layer follows the diurnal cycle of solar radiation and is shallow at sunrise, grows during the morning and afternoon and collapses after sunset. The solar heating of the surface causes air density differences that trigger unstable conditions in the boundary layer. As a result, convective turbulence is formed in the CBL. Such turbulent transport forms a well-mixed layer from its lower part, i.e. the surface, to the top of the CBL. This means that pressure-independent temperature, i.e. potential temperature, moisture or specific humidity and many other properties, such as $\mathrm{CO}_{2}$ mixing ratio, are very similar from the bottom to the top of the layer. For illustrative purposes, we will now focus only on the moisture distribution and evolution in the CBL. The CBL has usually a moist lower boundary, due to water bodies and vegetation at the surface, and a dry upper boundary. Contributing to maintain a well-mixed layer with such different properties at the boundaries, turbulence transports moisture from the lower to the higher levels. This turbulent transport is clearly visible through the updrafts. The updrafts, visible on the top-left panel of Figure 1.1, carry air that, being warmed up and moistened at the surface, is lighter than the air around and thus accelerates upwards in flame-like shapes. The top-left panel in Figure 1.1 shows the moist (blue) surface and the dry (red) air above the CBL, and how the updrafts, also called thermals, transport moisture to higher altitudes. The grey arrows in the figure, indicating the direction of the flow, show the air rising within the updrafts. The strongest updrafts reach as high as the CBL top and sometimes overshoot it, incorporating part of the air originally above the boundary 


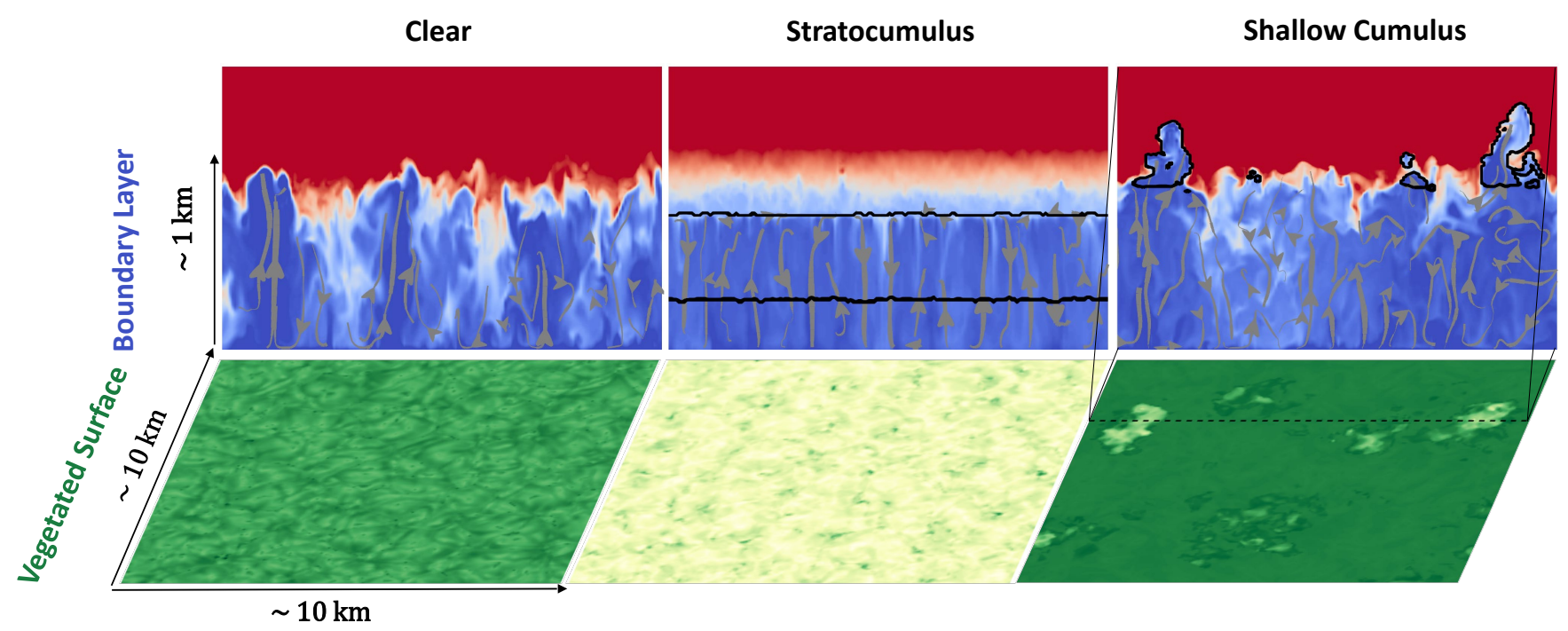

Figure 1.1: On the top, vertical cross-sections of the atmospheric boundary layer under three different situations obtained from simulations using the Dutch Atmospheric Large Eddy Simulation (see Section 1.2.5). On the top left, a clear sky boundary layer; on the top center a boundary layer topped by stratocumulus clouds; and on the top right, a shallow cumulustopped boundary layer. The blue color indicates moist air, and the red color dry air. Grey lines indicate the direction of the air flow. Both the stratocumulus deck as well as the shallow cumulus clouds are indicated by black contours. On the lower part the vegetation activity or photosynthesis is shown as calculated by the coupled land-surface model under the corresponding boundary layer. Yellow and green indicate low and high photosynthesis, respectively. Note that the green color scale has been stretched on the clear-sky surface (bottom left) compared to the shallow cumulus surface (bottom right) to show the wave-like structures in the clear-sky case due to non-radiative environmental effects. At the bottom right, the black dashed line across the surface indicates the location of the cross-section displayed above.

layer in the CBL. One could rightfully argue that such CBL is not well mixed. However, if we average along any horizontal direction over a long enough distance, or over long enough time, the heterogeneous and unpredictable location of individual updrafts balances and we observe a well-mixed "averaged" layer.

\subsubsection{The cloud-topped boundary layers}

When the temperature and water vapor at certain height reach condensation conditions, clouds form. In this thesis we deal with two types of boundary-layer clouds: shallow cumulus clouds (Duynkerke, 1998), characterized by their vertical development, and stratocumulus clouds (Duynkerke, 1998; Wood, 2012), with an indicative horizontal layering. The stratocumulus are considered part of the boundary layer because pressure-independent properties like liquid potential temperature and humidity are conserved and do not change significantly from the surface to the cloud top. This is visible at the top-center panel in 
Figure 1.1, showing a very similar blue intensity from surface to cloud top. In contrast to the clear sky CBL that was well mixed due to surface warming, the stratocumulus-topped boundary layer is well mixed due to the longwave cooling at the top. The larger emissivity of liquid water compared to water vapor combined with the typically higher temperature and larger moisture levels within the boundary layer explain the cooling at the cloud top, as emitted longwave radiation is larger than the received longwave radiation. Thus, air parcels, instead of being heated at the surface and rise as in the clear CBL, they cool at the cloud top and, being denser than the air around, sink down to the surface. This cooling creates a very sharp temperature gradient at the cloud top, a feature that is usually also present in the moisture profile. The top-center panel in Figure 1.1 shows the well-mixed moist air from the surface to the top of the cloud layer, and the drier air above. It also shows that stratocumulus, contoured by the black line, are very homogeneous, i.e a very large cloud deck, in its horizontal direction. Thus, stratocumulus clouds drive the flow within the boundary layer when they are present. The grey arrows indicate the circulation of air from the cloud top down to the surface. Provided enough supply of moisture and in absence of other physical factors, stratocumulus clouds continuously cool the boundary layer and, consequently, thicken by lowering the cloud base and lifting the cloud top. Key in our motivation to study the stratocumulus in this thesis is that they block a large share of the solar radiation reaching the surface. They do so quite homogeneously, as shown by the pale yellow colors at the surface.

In contrast, the shallow cumulus are a consequence of powerful and buoyant thermals in the CBL rather than the drivers of the flow. If the thermals are moist and warm enough, they will rise above the lifting condensation level. This level is the height at which the parcel cools down, due to ascent-related pressure decrease, to the point where the contained water vapor condensates. The condensation of water releases additional heat that further reinforces the buoyancy and rise of the thermal and, thus, the growth of the cloud. The top-right panel in Figure 1.1 shows how certain updrafts rising from the surface reach the lifting condensation level, create a cloud, indicated by black contours, and continue growing. Thus, we conclude that the shallow cumulus clouds are rooted in the surface and, following the parallelism, resemble an actual plant: although only the upper part is visible, its origin and structure extend far below their apparent base. This is also visible by the grey arrows indicating the movement of air from the surface through the updrafts up to the cloud. The shallow cumulus are heterogeneously distributed in space and, in contrast to the homogeneous stratocumulus, do not drive the flow in the boundary layer. Instead, the shallow cumulus are a consequence of the flow. Yet once they are formed they impact the boundary layer. Dynamically they transport moisture and other boundary-layer properties higher up through the ventilation of air, i.e. carrying moist and warm air from the subcloud to the cloud layer. In other words, cloud ventilation promotes the dryness of the subcloud layer. This ventilation is partly compensated by the subsiding air around cloud shells. But more important in this thesis is the fact that 
these clouds reflect part of the incoming solar radiation. Given their chaotic behaviour as part of the turbulent flow in the CBL, they introduce perturbations in the radiation field at the surface by shadowing some regions, due to direct blocking of the sunlight, and further illuminating others by lateral reflections. These clouds not only modulate the amount of light at the surface, but also the ratio of direct and diffuse radiation (Mercado et al., 2009). As we will see next, this is very relevant for the vegetation.

\subsubsection{The roots of the boundary layer over land: the vegetation}

At the lower boundary of the CBL lies the vegetated surface. The amount of energy available at the surface is the result of the balance between the total radiation arriving and being emitted or reflected. Most of the radiative energy surplus at the surface is distributed into heat transferred back to the atmosphere, called sensible heat flux, heat stored into lower layers of the surface, called ground heat flux, and heat used to evaporate liquid water to the atmosphere, called latent heat flux. Part of the latent heat flux consists of the water vapor released by plants during photosynthesis. Equating all these terms leads to the so-called surface energy balance (Katul et al., 2012; Moene and Van Dam, 2014). The plants, through the closing and opening of the stomata in their leaves, control the photosynthesis rates by incorporating atmospheric $\mathrm{CO}_{2}$ to the leaves and releasing water vapor that adds onto the latent heat flux. Plants optimize $\mathrm{CO}_{2}$ absorption and minimize water loss, regulating their stomata in a matter of minutes. Since the photosynthesis, and thus stomatal opening, depends on factors such as amount of light, wind at the surface, soil moisture, temperature, humidity and $\mathrm{CO}_{2}$ concentrations in the air, vegetation regulates the surface energy balance and is strongly coupled to the boundary layer: the heat and vapor released to the atmosphere depends on the activity of vegetation, but the vegetation activity also depends on atmospheric factors. The bottom panels in Figure 1.1 show the modeled photosynthesis rates at the surface, with green and pale yellow showing vegetation with high and low photosynthesis rates, respectively. The bottom-left panel shows how even at identical radiation conditions under clear skies, other factors such as near-surface turbulence have the potential to create wave-like patterns in the photosynthesis values. Due to the tight coupling between photosynthesis and evaporation, vegetation thereby also controls the latent and sensible heat fluxes. Although in this thesis we take all the environmental factors into account to calculate the vegetation responses, we focus mainly on shortwave radiation due to its large cloud-driven variability.

Besides the atmospheric factors, vegetation also shows different photosynthesis rates and reacts differently to environmental conditions depending on the plant type. Similarly, atmospheric conditions vary widely across the globe. For that reason it is interesting to investigate the vegetation coupling to the boundary layer and clouds in different regions of the world, with different ecosystems and climates. In Figure 1.2 we show the three 


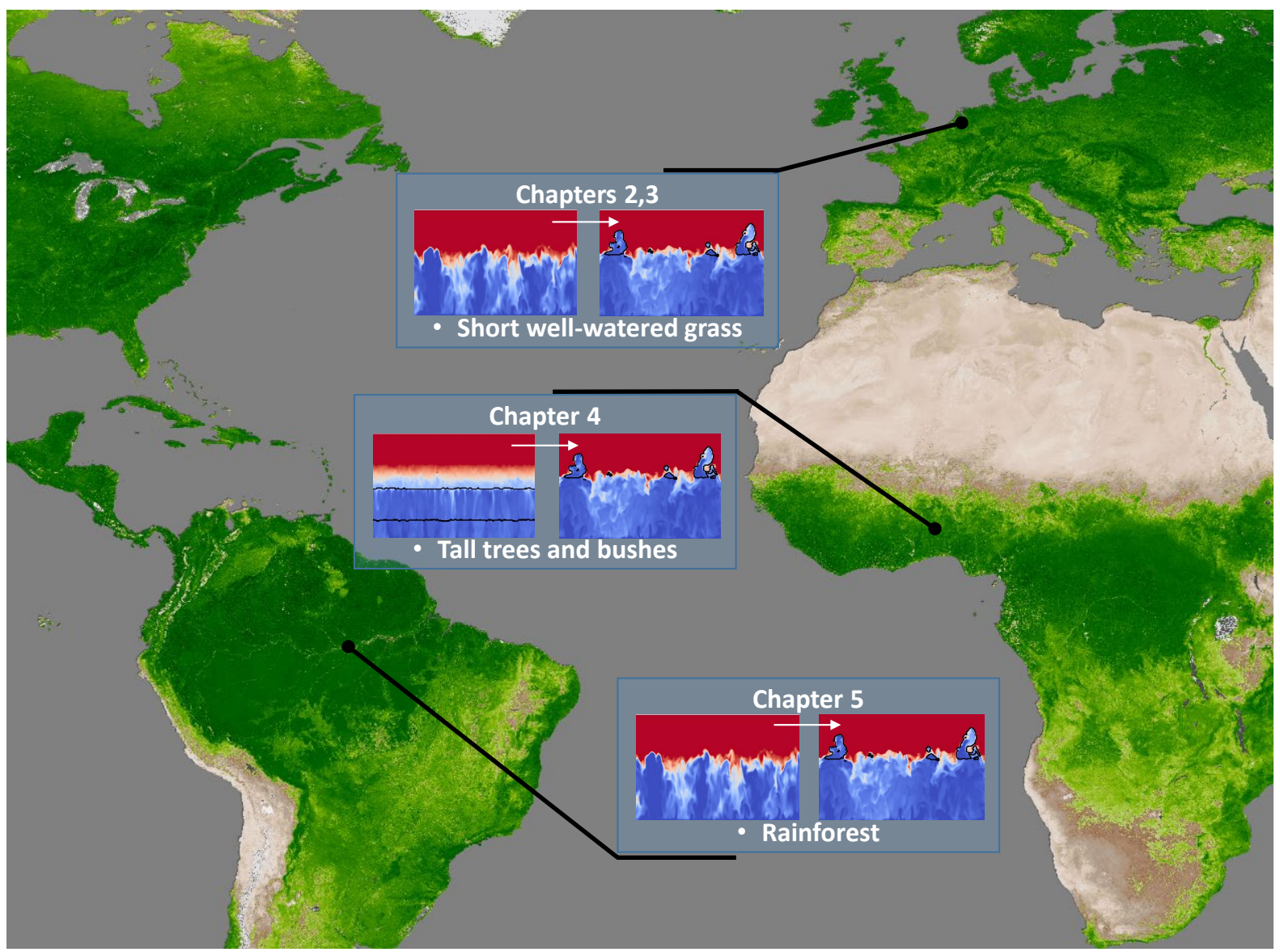

Figure 1.2: Overview of the locations, ecosystems and boundary-layer transitions studied in this thesis. Chapters 2 and 3 study the transition from a clear to a shallow cumulus-topped boundary layer and are based on moist and cool mid-latitude conditions over well-watered grass in The Netherlands. Chapter 4 deals with the stratocumulus to cumulus transition under the very warm and moist conditions over tall trees and bushes in Benin, southern West Africa. Chapter 5 treats the clear to shallow cumulus-topped boundary-layer transition on a moist and warm climate over the Amazonian rainforest in Brazil.

locations in the world where we base our studies. The first location is in the mid-latitudes, that is The Netherlands, where the dominant vegetation is well-watered grass and the climate is moist and relatively cool due to predominant westerly winds coming from the ocean. The remaining two regions studied are located in the tropics: one in Benin in southern West Africa, where the vegetation consists of high trees and middle-sized bushes and the climate is very moist and warm and conditioned by the seasonal monsoon coming from the Gulf of Guinea. The final study site is located in the Amazon, with an extensive high-tree rainforest and a moist but less warm climate compared to Benin due to the trade winds coming from the cooler Atlantic ocean. 


\subsubsection{Radiation as part of the boundary layer - surface coupling}

As we just showed, the vegetation is sensitive to many environmental variables, among which we focus on radiation. The reason is that radiation shows very rapid fluctuations, i.e. seconds to minutes, due to the presence of clouds. The radiation relevant for our studies is commonly divided in two parts: shortwave radiation (SW), the sun being its only source; and longwave radiation (LW), emitted by any object on the surface of the Earth and its atmosphere, including clouds. As discussed in Section 1.2.2, LW is critical in the maintenance and dissipation of stratocumulus and is mostly governed by temperature, which shows relatively slow variations, i.e. minutes to hours. Consequently, the largest perturbations in the timescales of our interest are caused by the rapidly varying and spatially heterogeneous shortwave radiation.

SW drives the diurnal cycle, the boundary-layer variations and, to a large extent, the plant photosynthesis along the day. Due to solar radiation, the coupling between clouds and surface energy fluxes intensifies: under the presence of shallow cumulus, radiation heterogeneities at the surface impact the updrafts on which clouds are rooted. For situations with the sun overhead the result is a weakening of the updraft that lead to the formation of the cloud, as the cloud casts its shadow on the surface on which the cloud and the updraft are rooted. We illustrate this coupling by showing the lower vegetation photosynthesis below clouds in the bottom-right panel in Figure 1.1. In the case of stratocumulus, absorption of SW radiation weakens the cloud top longwave cooling, thinning the cloud layer and increasing the surface fluxes. Since the amount of light is decreased horizontally homogeneous and in large amounts by stratocumulus, photosynthesis at the surface shows homogeneous and low values. This is visible in the bottom-center panel of Figure 1.1. Although not visible in the figure, a relevant process deserves our attention: in addition to modifying SW at the surface, clouds alter the direct-diffuse ratio of SW that is very relevant for vegetation. Direct radiation, coming directly from the sun, does not penetrate deep in the canopy and saturates the photosynthesis of the leaves at the canopy top. Diffuse radiation results from scattering and reflection of sunlight not only by clouds but also aerosols, molecules in the air and leaves themselves. It penetrates further to the canopy as it comes from all possible directions. As a result, the vegetation canopy is known to do more photosynthesis under diffuse than direct radiation everything else equal (Kanniah et al., 2012). This, according to the lower panel of Figure 1.1, leads to increased photosynthesis under very thin clouds, as visible from the darker spots at the edges of large clouds or under small clouds. Due to the coupled $\mathrm{CO}_{2}$ uptake and water vapor release by photosynthesis, a similar increase in latent heat flux under thin clouds is theoretically predicted. Such a property, not present at leaf level but appearing when we upscale the explicit processes, is referred to as an emergent property. This inevitably impacts the $\mathrm{CO}_{2}$ budget and the latent heat flux and, thus, the surface energy balance. We study this in more detail in Chapter 3. 


\subsubsection{Simulating the boundary layer - vegetation interactions with LES}

The graphics shown in Figure 1.1 are a visualization of simulations done with a numerical technique called Large Eddy Simulation (LES). It is also our main tool for the study and analysis of the boundary layer and its interaction with the surface. LES are a class of numerical models that simulate, using first principles at the resolvable scales, the behavior of the turbulent flow in the boundary layer. LES resolve the time evolution in a three-dimensional field of the approximated and filtered version of the equations that assume the conservation of mass, energy or heat, momentum (known as Navier-Stokes equation) and other scalar such as specific moisture or $\mathrm{CO}_{2}$ mixing ratio (Moeng, 1998). The dynamic components of LES models have been thoroughly tested against observations and are frequently used as virtual laboratories that allow controlled and systematic experiments that otherwise would be impossible in the real atmosphere. The main advantage of LES compared to other atmospheric models is that LES explicitly resolve the atmospheric turbulent motions and enable us, as we did in Figure 1.1, to simulate and quantify features such as individual turbulent updrafts and clouds. A novelty in this thesis is that we couple the LES to a land-surface model that mimics the physiological responses of vegetation to environmental conditions including near-surface air motions, soil moisture, $\mathrm{CO}_{2}$ concentration, temperature, humidity in the air and available direct and diffuse shortwave radiation. This vegetation submodel simulates in a mechanistic manner the opening and closing of stomata depending on the environmental conditions. These conditions control the stomatal aperture and subsequent photosynthesis, through which $\mathrm{CO}_{2}$ and water vapor are exchanged between the vegetation and the atmosphere. By simulating the effects on the stomatal aperture of leaves, it is radiation that creates the patches with low photosynthesis under shallow cumulus clouds (see bottom-right of Figure 1.1), and it is the turbulent air motions that cause the wave-like shapes under clear skies (bottom-left panel of Figure 1.1).

Mesoscale models, used typically for forecasting or accounting for larger scales and domains, simulate the effects of turbulence and cloud dynamics through parameterizations instead of resolving them explicitly (Stensrud, 2011; Powers et al., 2017). Parameterizations are computationally less intense approximations of the resolved processes in LES, such as turbulence and cloud dynamics, that seek to reproduce their main effects at the scales the model is simulating. Thus, they attempt to gain computational efficiency at the cost of detail and realism on the dynamic processes involved at the small spatiotemporal scales in the order of meters and seconds. In this thesis we systematically analyze whether the state-of-the-art parameterizations in these mesoscale models are able to simulate the shallow-cumulus-topped boundary layer and its interaction with radiation and surface in Chapter 2.

As we mentioned before, LES models resolve with high realism the flow dynamics in the atmosphere. This contrasts with the radiative and surface processes, imperatively param- 
eterized due to their properties varying on the sub-meter scales with high complexity and, consequently, computational cost. The surface-vegetation scheme employed and further developed in this thesis consists of a land-surface model with a biological-mechanistic representation of vegetation, where the stomata regulate their aperture depending on the environmental factors already mentioned. Similarly, it is unfeasible with current computational power to simulate the path and interactions of every photon along its travel through the boundary layer, its clouds, and the vegetation. Most LES models make use of one-dimensional radiative transfer schemes, allowing radiation to travel only upwards or downwards and neglecting any horizontal transfer of light. Thus, our methodology lies within the state-of-the-art capacities. Currently, however, efforts are made towards using the full 3-dimensional radiative transfer in LES. This involves the development of a scheme allowing light to travel in the three spatial dimensions. Under these conditions real features like dynamic tilting of the cloud shades or lateral reflections of light by clouds become feasible. This new treatment of radiation inevitably affects the surface and, potentially, the boundary layer development. We study the impact of this novel approach to radiative transfer on vegetation, clouds and boundary-layer dynamics in Chapter 5.

Summarizing, in this thesis we design and perform Large Eddy Simulation experiments where we couple the dynamics of the boundary layer, the perturbed radiation by clouds, and the vegetated surface. Here we study properties of the three boundary layer types, i.e. cloudless, and topped with shallow cumulus or stratocumulus. We put special emphasis on how one type of boundary layer evolves in another as part of the diurnal cycle, and what is the role played by the vegetation in this transitions. We study the transition from clear to shallow cumulus in Chapters 2, 3 and 5, and the transition from a stratocumulus to shallow cumulus-topped boundary layer in Chapter 4. The boundary layer transitions are indicated in Figure 1.2 with the corresponding geographical location of each chapter.

\subsection{Background and state of the art on cloud - vegetation interactions research}

The study of vegetation activity and its link to evapotranspiration regulating the surface energy balance and the subsequent coupling with the boundary layer development has only been studied as an integrated system in the last three decades. As a consequence, most of previous literature on the topic deals with either vegetation responses to atmospheric forcings, or cloud dependencies on surface moisture supply without considering the explicit role of vegetation. This lack of cohesion in scientific literature, particularly recurrent for observational studies, shows the need for more first-principle based integrating research as the one performed along this thesis. 
One of the first studies realizing that the atmosphere influences the evaporated water at the surface and, thus, the surface energy balance was Priestley and Taylor (1972). Although they proposed empirical relations between evaporation and the available energy at the surface, they did not explore the physical processes that drive the relation. This task was first tackled by de Bruin (1983). In his work, he proposed to couple an already existing simplified land-surface model to a mixed-layer model representing the atmospheric boundary layer (ABL) under convective conditions, intending to explain and explore the atmospheric processes involved in the partition of the available energy at the surface as proposed by Priestley and Taylor (1972) on a diurnal scale. Jacobs and De Bruin (1992) further extended this framework by coupling the surface fluxes to a mixed-layer model of the atmosphere and showed the potential feedbacks arising. This was later extended to the effects of atmospheric $\mathrm{CO}_{2}$ on vegetation (Jacobs and de Bruin, 1997), which plays a role in the energy partitioning at the surface (Katul et al., 2012).

\subsubsection{Observing the cloud - vegetation interactions}

The observations in later years further reinforced the idea of a coupled land-atmosphere system with vegetation playing an active role. Within this system, the interactions between clouds and active vegetation received the attention by Freedman et al. (2001). Based on observations of an oak forests and boundary-layer clouds above, they showed that the transpiration by active vegetation conditioned the ABL height as well as the lifting condensation level of clouds along the year. Analyzing a 13 year observational dataset Zhang and Klein (2013) further reinforced the idea of surface fluxes affecting cloud properties: they found that surface fluxes influenced the thickness of shallow cumulus clouds on daily scales. Showing the reverse impact, i.e. the effect of clouds on vegetation, Freedman et al. (2001) found indications of larger and more efficient uptake of $\mathrm{CO}_{2}$ by the forest on days when cumulus clouds were present. The idea that this was due to the enhancement of diffuse radiation by clouds was explored, among other, by Min and Wang (2008). Using data gathered on a mid-latitude hardwood forest, they found clouds to be the most relevant climatic factor for vegetation activity. In particular, they reported clouds to increase the radiation use efficiency of carbon uptake by plants, in addition to moderate cloud covers yielding maximum vegetation $\mathrm{CO}_{2}$ uptake. They argued that the clouds impacted radiation at the surface by not only diminishing the total amount of shortwave radiation, but also by increasing the diffuse to direct ratio due to the cloud disturbances on radiation. A broad range of similar results confirming the enhanced plant activity due to diffuse radiation, i.e. the diffuse fertilization effect, is gathered in Kanniah et al. (2012). These hypotheses were recently reinforced through satellite retrievals by Cheng et al. (2016), who quantified the regional radiative effects of clouds through their optical depth. They found regionally enhanced light-use efficiency by vegetation for low optical depths, although overall clouds did not lead to increased photosynthesis given the 
larger effect in total radiation attenuation

\subsubsection{Modeling the cloud - vegetation interactions}

\subsubsection{The surface-boundary layer exchange}

The modeling of vegetation-boundary layer interactions started, as stated before, with the pioneering study by de Bruin (1983). A relevant feature on these modeling studies is whether the biophysical processes and their couplings are explicitly modeled or parameterized. The radiation and responses of vegetation to atmospheric forcings and possible feedbacks are, due to the high complexity and small scale of the processes involved, always parameterized. Thus, we classify the modeling studies as explicit or parameterized by focusing on whether the turbulence and cloud dynamics in the boundary layer are explicitly resolved or not. Among the latter, the effect by the partitioning between sensible and latent heat flux on the boundary layer at sub-daily scales has been extensively studied. One of the most representative studies is the one by Ek and Mahrt (1994) further developed by Ek and Holtslag (2004), where they used the relative humidity tendency as a proxy for cloud formation using a coupled land-surface model and a mixed-layer framework. A more comprehensive study on the effects and feedbacks between the boundary layer and the surface evaporation was presented by van Heerwaarden et al. (2010). This study was extended by van Stratum et al. (2014), who incorporated effects of convective clouds and explicitly presented the dynamic impact and feedbacks of such clouds in the subcloud layer and, potentially, at the surface.

\subsubsection{Vegetation as regulator of the cloud-surface coupling}

Only recently the explicit link between surface fluxes and vegetation including the carbon cycle attracted the attention of researchers. At global scale and coarse resolutions, i.e. $\sim 250 \mathrm{~km}$ Cox et al. (2000) performed the first climate sensitivity tests incorporating the impact of the carbon cycle on the surface fluxes and meteorology, focusing on their global inter-decadal effects. The research by Mercado et al. (2009) further motivated the need to link the global carbon budget to meteorology, with special emphasis on the directdiffuse partitioning of radiation. Using a combination of observations and global modeling they found indications that increases in diffuse radiation due to increased cloud cover or aerosols had a significant impact on the land carbon sink worldwide over the last century. More recently and at a regional and monthly scale, Boussetta et al. (2013) implemented a modified version of the plant model A-gs employed in this thesis and proposed by Jacobs (1994) and Ronda et al. (2001) in the land-surface model part of the three-dimensional European Center for Medium-Range Weather Forecast (ECMWF) atmospheric model. Imposing numerical weather forecast resolutions, i.e. $\sim 50 \mathrm{~km}$, Boussetta et al. (2013) 
found a reduced errors not only in the estimation of atmospheric $\mathrm{CO}_{2}$ budget and its variability, but also in evapotranspiration estimations. A similar coupling between the A-gs model by Jacobs (1994) and, in this case, a mixed-layer model representing the diurnal evolution of the convective boundary layer lead Vilà-Guerau de Arellano et al. (2012) to suggest that the future enhancement of atmospheric $\mathrm{CO}_{2}$ will cause a decrease in cumulus clouds in the boundary layer. The above mentioned studies crudely represent the canopy structure and their effects. Thus, the growing interest in properly simulating the vegetation responses requires a better representation of the in-canopy processes. Similar to clouds, the vegetated canopy strongly affects the partitioning of direct and diffuse radiation. The transfer of radiation within the canopy is commonly represented as a one-dimensional transfer in a horizontally homogeneous canopy, with Baldocchi et al. (1985) presenting a complete formulation and validation of such a model framework in an oak forest. In parallel to recent attempts to include three-dimensional features in cloudradiation interactions, current work is directed towards simulating a much more realistic three-dimensional radiation transfer inside the canopy, as proposed by Kobayashi et al. (2012).

\subsubsection{Simulating turbulence and clouds explicitly to study the coupling: LES}

Regardless of the coupling between $\mathrm{CO}_{2}$ and atmospheric dynamics within the boundary layer, the parameterized representations of clouds and turbulence in three-dimensional mesoscale and climate models lead to errors in the radiation fluxes and, consequently, the surface fluxes driving the boundary layer on a diurnal scale. Examples of this are the work by Knippertz et al. (2011) and Hannak et al. (2017). They showed how global climate models, with spatial resolution in the order of $100 \mathrm{~km}$, fail to adequately represent low clouds in southern West Africa. This is especially apparent during the night and early morning, leading to substantial biases in the regional radiation budget and development of the boundary layer during the day. A similar problem was found and tackled by Jimenez et al. (2016b), in this case for North America and on a smaller scale of $10 \mathrm{~km}$. By parameterizing the radiative effects of clouds smaller than the model resolution, Jimenez et al. (2016b) reduced significantly the biases in surface irradiance across the whole USA. Unfortunately they did not study how such biases, or reductions of it, impacted the boundary layer properties and cloud formation.

\section{Coupling the surface and the boundary layer}

The use of Large Eddy Simulations techniques set the path for the explicit modeling of boundary-layer turbulent flows on very fine spatio-temporal scales as small as meters and seconds. On the use of LES as a tool to investigate cloud-atmosphere-surface interactions, 
the first step was given by Sommeria (1976) by introducing in LES the moist thermodynamics allowing cloud formation, with a relevant contribution thereafter by Cuijpers and Duynkerke (1993). On the other end, the first study resolving the turbulent motions of the clear boundary layer with a coupled land-surface model including $\mathrm{CO}_{2}$ effects was Albertson et al. (2001). Following previous work, Patton et al. (2005) improved the coupling between soil and the atmosphere and documented land-heterogeneities to have the ability to generate circulations within the boundary layer, with a strength depending on the scale of the heterogeneities at the surface. Many studies followed, aiming to further develop the theory on the role played by static heterogeneities, i.e. heterogeneities due to the intrinsic nature of the surface, such as the ones by Huang and Margulis (2010) and van Heerwaarden et al. (2014). The former work underlined the relevance of atmosphere-surface feedbacks, showing discrepancies of up to $18 \%$ in surface sensible heat flux if neglected. The latter developed general scaling laws to explain the relation between heterogeneity size, the scale of induced secondary circulations and the consequent entrainment in the CBL.

\section{Cloud - topped boundary layers: introducing the radiation effects}

Meanwhile, Schumann et al. (2002) performed the first LES study on the impact by cloud shading on boundary-layer properties. Cloud shading can be viewed as a dynamic heterogeneities, as cloud shadows introduce transitory spatial inhomogeneities at the surface affecting the surface fluxes. Another example of dynamic heterogeneity is, for example, the soil moisture variation due to precipitation (Rieck et al., 2014). Although a simplified study in some aspects, Schumann et al. (2002) provided the first insights on the radiative effects of clouds in the boundary layer. They found cloud shading to reduce the turbulence kinetic energy (TKE) as well as the dominant length scales within the boundary layer. They also reported the solar angle and, thus, the inclination of cloud shades to have little impact. This issue regained attention in recent years with the appearance of several related studies. Lohou and Patton (2014) studied a shallow cumulus case on LES coupled to a land-surface model, quantifying shade-driven average and local increases of about $3 \%$ and $30 \%$ respectively in the evaporative fraction, as well as larger surface flux variability. Yet they found these differences not to impact entrainment rates at the boundary layer top. Almost simultaneously, Vilà-Guerau de Arellano et al. (2014) presented another shallow cumulus case with the vegetation A-gs submodel (Jacobs, 1994) incorporated to the land-surface model. Their work showed a decrease in slab average liquid water path when shading was considered. The result was explained by the reduced slab average surface fluxes. They further quantified rapid surface flux and carbon uptake fluctuations due to cloud shadings. Such rapid surface flux oscillations due to cloud shading were measured with a 1 minute resolution using scintillometry techniques by van Kesteren et al. (2013). Recently Kivalov and Fitzjarrald $(2018,2019)$ performed a rigorous classification of such 
observed cloud-driven rapid fluctuations of radiation at the surface and the surface flux responses. Interestingly, Vilà-Guerau de Arellano et al. (2014) also found vegetation functional types characterized by different photosynthesis cycles, i.e. C3 and C4, to provide distinct surface flux responses. These fluxes were found to feed back to the atmosphere causing different boundary layer heights as well as cloud bases depending on the plant type. Horn et al. (2015) extended on this work, although without coupling to $\mathrm{CO}_{2}$. They reported a decreased TKE with cloud shading, in agreement with Schumann et al. (2002), and more interestingly, a reduction in boundary-layer length scales due to the localized shading of the clouds. As a consequence, the coupling to the surface modified cloud spatial variability.

The most recent studies include a study by Gronemeier et al. (2016) who further explored the potential of tilted shading to induce secondary circulations. Contrary to Horn et al. (2015), who described opposed thermal circulations and weaker updrafts due to shading, Gronemeier et al. (2016) found reinforcing secondary circulations leading to stronger updrafts triggered by cloud shading, with a magnitude varying with the zenith angle. They furthermore identified increasing zenith angles to favour larger and longer-lived clouds. Using for the first time a three-dimensional radiative transfer scheme in LES, and a simplified land-surface model, Jakub and Mayer (2017) showed the creation of cloud streets purely driven by cloud-induced dynamic heterogeneities at the surface. Most of the mentioned studies focused on idealized windless conditions with an immediate surface response to reveal the effects of dynamic heterogeneities. A weak wind was found to enhance the shading effects in the case researched by Horn et al. (2015), similar to the enhanced cloud street reported by Jakub and Mayer (2017) when effects of wind and solar angle coincided. However, Sikma et al. (2018) found wind or a lag in vegetation response to environmental conditions to weaken the coupling between the surface and the boundary layer with a small impact due to compensating effects. Thus, the processes leading the cloud-vegetation coupling under more realistic conditions, such as wind, lag in vegetation response or $3 \mathrm{D}$ radiation still remain not fully understood.

As explained in the studies mentioned above, most of the cloud-land investigations so far were based on case studies with cumulus clouds. However, stratocumulus clouds are also present over land worldwide (Eastman and Warren, 2014), attracting so far less attention from the scientific community. To tackle this knowledge gap, a European-African partnership with the participation of more than 16 universities and research centers carried out the Dynamics-aerosol-cloud Interactions in West Africa (DACCIWA) project. This project included an intensive 2-month meteorological measurement campaign in southern West Africa, in which I participated. The stratocumulus clouds have been subject of numerous LES studies since the seminal work by Deardorff (1980), but almost always under marine conditions. The impact of stratocumulus over land, however, is not negligible as shown in Figure 1.1, where photosynthesis and, consequently, surface fluxes decrease largely. The only LES studies on stratocumulus over land to our knowledge are the ones 
by Mechem et al. (2010) and Ghonima et al. (2016). The first of these studies simulated a post-frontal stratocumulus case with prescribed surface fluxes throughout the simulation, thus not allowing for any coupling to the surface. More complete is the study by Ghonima et al. (2016) who quantified, using a mixed-layer model complemented by LES, the factors leading to stratocumulus thinning both over sea and over land near the USA Eastern coast, where stratocumulus are advected from the Pacific ocean. They further studied the effects of different Bowen ratios, i.e. the ratio between sensible and latent heat flux, in the stratocumulus thinning and found dry surfaces characterized by large (low) sensible (latent) heat fluxes to dissipate the cloud layer soon after sunrise, while moist surfaces would actually thicken the cloud layer. However, no study so far has explored the stratocumulus-land interactions with clouds originated over land or with a more realistic land-surface model. Such surface model would account for non-linearities in the surface flux partitioning and, thus, varying Bowen ratio. In this context, we perform in Chapter 4 a LES experiment on the stratocumulus to cumulus transition over land with a landsurface model including vegetation related processes, and characterize such transition and the driving physical processes. To design and evaluate the study and validate it we made use of the extensive dataset gathered during the DACCIWA field campaign.

Summarizing, in this thesis we address current questions in the field of cloud-surface interactions employing a LES model coupled to a land-surface model mimicking the vegetation response to environmental variations in the order of seconds. Taking as a reference systematic LES simulations, we assess and quantify the ability of current mesoscale models in simulating shallow convective clouds and their dynamic and radiative impacts in Chapter 2. We further explore the response of vegetation to cloud-induced variations in radiation and its direct-diffuse ratio in Chapter 3. We extend the current knowledge on stratocumulus to cumulus transition over land, and the role played by vegetation, by fully characterizing and analyzing the physical drivers of such transitions in the southern West African climate (see Figure 1.2) in Chapter 4. Finally, Chapter 5 explores the forthcoming development of three-dimensional radiative transfer schemes in LES simulations by analyzing and quantifying the impact of 3D radiation on cloud-vegetation interactions and boundary layer properties on an Amazonian shallow cumulus case.

\subsection{Outline of this thesis}

The most relevant processes studied in this thesis are schematically depicted in Figure 1.3. In this figure we illustrate the processes and situations studied in each of the remaining chapters of this thesis. Our research strategy is based on decomposing the overarching aim of this thesis (see box in Section 1.1) in building blocks represented by questions that we address in individual chapters. To answer the individual questions we systematically design Large Eddy Simulation experiments to break down the complexity of the processes 

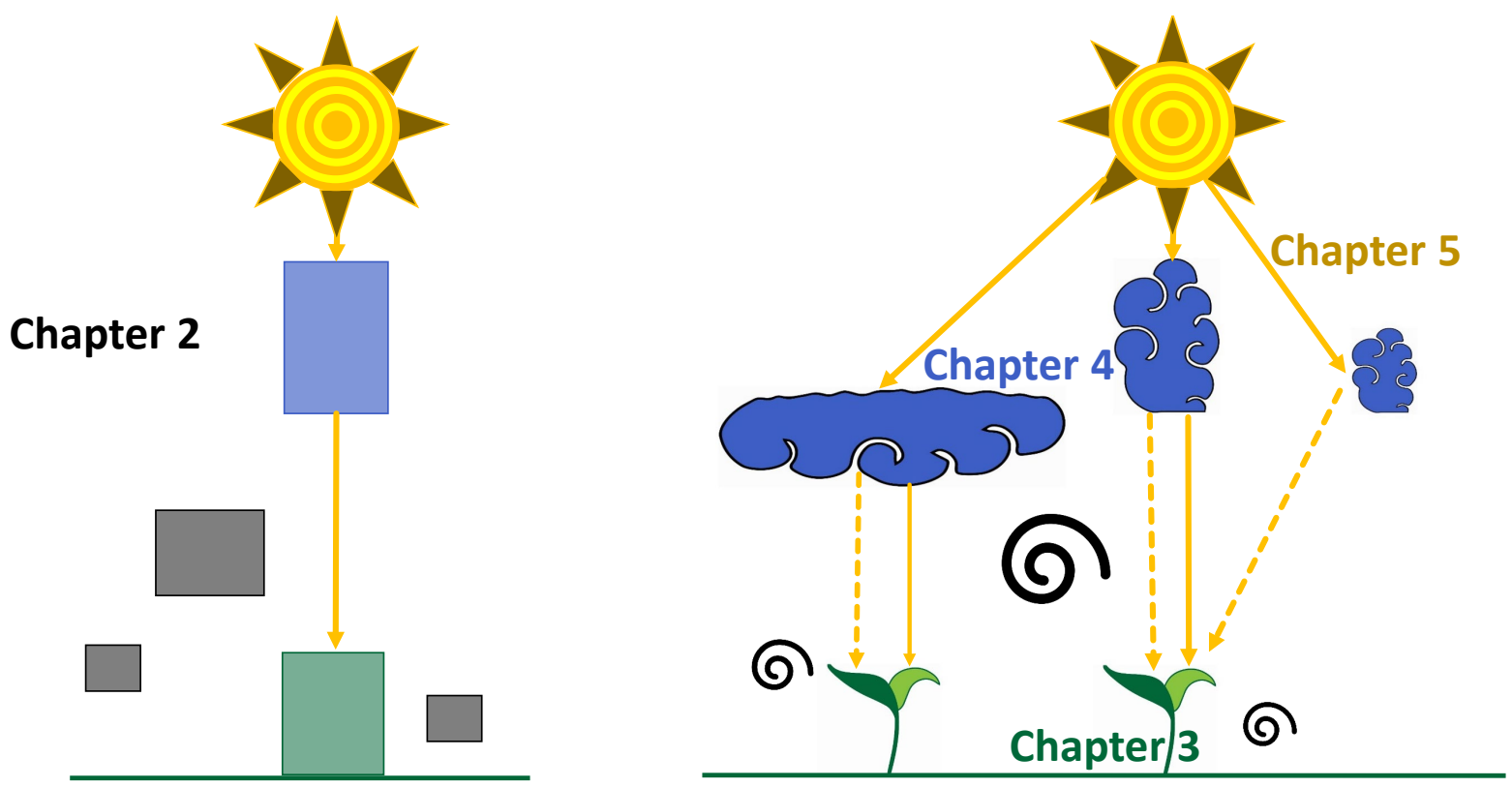

Figure 1.3: Conceptual pictogram of the processes and couplings considered in this thesis and the corresponding chapters. Chapter 2 studies the ability of parameterizations in mesoscale models to represent shallow cumulus onset and its dynamic and radiative effects at the surface. Chapter 3 researches the impact of direct and diffuse radiation and their cloud-induced variations on the vegetation and its feedback to the boundary layer and clouds. Chapter 4 deals with the transition from stratocumulus to cumulus-topped boundary layer over land and the role of the vegetated surface in it. Chapter 5 investigates the effects of three-dimensional radiation on a shallow cumulus topped boundary layer with a coupled vegetation model at the surface.

studied and to quantify them. The following four research questions are tackled along Chapters 2,3,4, and 5 .

Are current parameterized calculations of shallow cumulus in weather forecasting models adequate to represent how clouds interact with the surface and the boundary layer? Can we define a research strategy to diagnose the misrepresentations?

Most of the work presented in this thesis is carried out by Large Eddy Simulations of the boundary layer dynamics and its interaction with radiation and the vegetation at the surface. However, one may wonder whether such explicitness and accuracy to represent the cloud dynamics and its interactions is truly needed. To answer this question, in Chapter 2 we explore whether current mesoscale models adequately represent cloud processes and their impact on the boundary layer and the surface. To that end we use a state-of-the-art mesoscale model with parameterized turbulence and the most recently developed parameterizations for shallow convection. We compare the results to two explicit simulations by different LES models. The idealized case study is based on a day with 
shallow cumulus clouds over homogeneous grassland in the mid-latitudes. The radiative and thermodynamic effects of cloud representations on the surface, subcloud and cloud layers are studied. We further analyze the impact of these clouds on radiation and at the surface, focusing on the spatial distribution of clouds, cloud thickness and the related radiation at the surface. We conclude that current parameterizations in mesoscale models are not able to properly represent the onset, vertical development and horizontal spread of shallow convection nor their dynamic and radiative effects.

What is the effect of cloud-driven radiative perturbations, including the direct and diffuse partitioning, on the vegetation responses? Do these surface responses feed back to the boundary layer and clouds?

We further quantify the cloud-vegetation interactions on a shallow cumulus day in the midlatitudes using the LES technique in Chapter 3. In this chapter we put special emphasis on the role played by clouds and vegetation in the direct and diffuse partitioning of shortwave radiation. By implementing a leaf-to-canopy upscaling that includes sunlit and shaded leaves as well as different penetration rates for direct and diffuse light, we analyse the response of vegetation and surface fluxes to cloud-driven perturbations in radiation and its direct-diffuse ratio. We further quantify the main processes within the plantmechanistic model that lead the cloud-induced variations in carbon uptake by vegetation. Finally, we explore whether the different vegetation response to clouds are strong enough to feed back to boundary layer properties and, consequently, to clouds. Results show that the direct-diffuse partitioning produces new features in vegetation response and, thus, surface flux regulation. However, these modifications at the surface are not strong enough o feedback to the boundary layer.

What are the radiative and dynamic characteristics of a stratocumulus to cumulus transition over land? What is the role played by local physical processes, including the vegetation responses?

The transition from clear sky to shallow cumulus studied in Chapters 2 and 3 is only one of the boundary layer transitions where vegetation and clouds interact. Another very frequently studied transition is the stratocumulus to cumulus transition. Yet almost all previous studies deal with such transitions in marine conditions, leaving the transition over land remarkably unexplored so far. This transition is typically misrepresented by mesoscale and climate models, with strong implications in the radiation budget during the day. Recognizing the larger magnitude and variability of surface fluxes when over land, we perform in Chapter 4 an idealized LES simulation of the stratocumulus to cumulus transition over tropical vegetation in southern West Africa. Inspired by observations from the DACCIWA campaign in Benin we design a LES case and characterize the transition effects on turbulence and thermodynamics, surface and radiation. We further identify the physical processes, including the surface fluxes regulated by vegetation, relevant for the thinning of the stratocumulus cloud deck and subsequent break up. We do this for a 
windless case as well as for two different wind conditions representative of the observed wind during the experimental campaign. We finally explore how variables used by larger scale models behave along the transition for all the experiments. The simulated transition shows a evolution from an initial regime typical of nighttime marine stratocumulus to a cloud-topped convective boundary layer. We find that the surface is key in the transition, with the necessary contribution of shortwave radiation.

What are the differences between using one-dimensional radiation compared to a more realistic three-dimensional radiation on a shallow cumulus-topped boundary layer with active vegetation? Do the surface, boundary layer and cloud properties change?

Almost all research in literature, as well as the previously mentioned chapters in this thesis, make use of one-dimensional radiative transfer schemes. This is a clear limitation as radiation is not allowed to travel in any horizontal direction and poses strong consequences in the response of the vegetated surface to cloud-induced radiation perturbations. Features such as tilting of cloud shades or light reflections by lateral faces of clouds are neglected in one-dimensional radiative schemes. Using an innovative and relatively fast three-dimensional radiative transfer solver coupled to LES we explore the limitations of using the common one-dimensional radiation in Chapter 5. In this case we base the surface and atmospheric conditions of our numerical experiments on the Amazonian rainforest. The purpose of this chapter is, thus, to reveal the implications and limitations of using a one-dimensional radiative scheme on a day with shallow cumulus, where the cloud shading and light reflection may be of relevance for the evolution of the vegetation and surface fluxes, boundary layer and clouds. We first examine the new features appearing at the surface when using a three-dimensional radiation scheme. Afterwards, we study the differences in turbulence and thermodynamic properties within the boundary layer, and the effects they have on cloud spatial characteristics and dynamics due to the distinct response of the vegetation at the surface. Results show a wider range of direct and diffuse radiation combinations at surface, reflected in the surface fluxes. Furthermore, the shadetilt appearing in three-dimensional experiments allows the development of more buoyant updrafts leading to more active and larger clouds.

Finally, Chapter 6 completes the thesis with a summary of the main conclusions based on our findings and an outlook including recommendations for future work on how to continue the research. 


\section{2}

\section{Shallow cumulus representation and its interaction with radiation and surface at the convection gray zone}

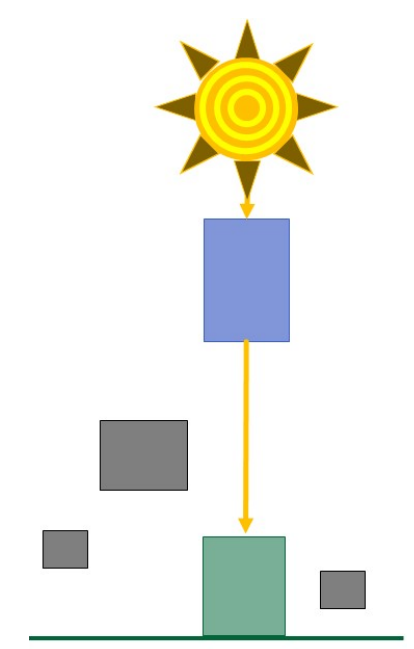

This chapter is published as:

Pedruzo-Bagazgoitia, X., Jiménez, P. A., Dudhia, J., and Vilà-Guerau de Arellano, J. (2019a). Shallow cumulus representation and its interaction with radiation and surface at the convection gray zone. Monthly Weather Review, 147(7):2467-2483 


\section{Abstract}

This study presents a systematic analysis of convective parameterizations performance with interactive radiation, microphysics and surface on an idealized day with shallow convection. To this end, we analyze a suite of mesoscale numerical experiments, i.e. with parameterized turbulence. In the first set, two different convection schemes represent shallow convection at a $9 \mathrm{~km}$ resolution. These experiments are then compared with model results omitting convective parameterizations at $9 \mathrm{~km}$ and $3 \mathrm{~km}$ horizontal resolution (gray zone). Relevant in our approach is to compare the results against two simulations by different Large Eddy Simulation (LES) models. Results show that the mesoscale experiments, including the $3 \mathrm{~km}$ resolution, are unable to adequately represent the timing, intensity, height and extension of the shallow cumulus field. The main differences with LES experiments are: a too late onset, too high cloud base and a too early transport of moisture too high, overestimating the second cloud layer. Related to this, both convective parameterizations produce warm and dry biases of up to $2 \mathrm{~K}$ and $2 \mathrm{~g} / \mathrm{Kg}$, respectively, in the cloud layer. This misrepresentation of the cloud dynamics leads to overestimated shortwave radiation variability, both spacewise and timewise. Domain averaged shortwave radiation at surface, however, compares satisfactorily with LES. The shortwave direct and diffuse partition is misrepresented by the convective parameterizations with an underestimation (overestimation) of diffuse (direct) radiation both locally and, by a relative $40 \%$ $(10 \%)$, of the domain average. 


\subsection{Introduction}

Shallow convective clouds disturb non-linearly the coupling of several processes within the atmosphere (Arakawa, 2004). The most relevant processes are the coupling between dynamics and radiation through light absorption, reflection and scattering by cloud droplets (Liou, 2002), the interaction between dynamic and microphysical processes by moisture and latent heat transport through the atmosphere (Grabowski, 2014); and the surfaceatmosphere coupling by altering the radiation budget at the surface (Trenberth et al., 2009) and subsequently creating dynamic heterogeneities (Gronemeier et al., 2016; Jakub and Mayer, 2017).

Due to current limitations on simulating explicitly all the relevant scales and processes, convective parameterizations are used to represent the cloud dynamics. Similarly, the representations of radiation and surface processes need to be described in a parametric form. Convective parameterizations are developed as stand-alone representations and are rarely tested in combination with other interactive processes. The works of Lenderink et al. (2004) and Couvreux et al. (2105) are representative studies of convective parameterizations on daily scales. However, none of them take into account possible radiative effects of the parameterized clouds on the atmosphere nor at the surface. By prescribing radiative tendencies and surface fluxes, instead, they omit potential interactions and feedbacks relevant to the convection representation. Similarly, Guichard et al. (2004) did consider the effects of convection on radiation, but not the dynamic heterogeneities created at the surface.

Focusing first on turbulence-resolving Large Eddy Simulation (LES) in the scale of 50 $\mathrm{m}$, previous studies have shown the relevance of the interaction between shallow cumulus $(\mathrm{ShCu})$ and surface turbulent fluxes on diurnal scales: Horn et al. (2015) discussed how the explicit representation of cloud shading creates a larger population of shorter-lived and smaller clouds. In a series of systematic experiments with different assumptions on the surface representation, Sikma et al. (2018) found that spatially homogeneous noninteractive surface fluxes yield a cloud cover decrease of between 5 and $10 \%$ during the period of strongest convection. The coupling also influences the transport of moisture. It is reduced by more than $50 \%$ if cloud shading is neglected, and by up to $41 \%$ if the shading-related radiative effects are not treated locally (Sikma and Vilà-Guerau de Arellano, 2019). Jakub and Mayer (2017) and Klinger et al. (2017) showed that cloud fields may have different morphology if the three-dimensional shortwave and longwave radiative effects, respectively, of clouds are included. All this evidence shows the need to include cloud-induced surface heterogeneities to investigate the impact on shallow convective parameterization studies.

Regarding models in which turbulence and moist convection are parameterized, i.e. mesoscale models, Jimenez et al. (2016a) studied the radiative effects of parameterized 
shallow convection at $9 \mathrm{~km}$ horizontal resolution using the mesoscale Weather and Research Forecasting model - Solar (WRF-Solar) (Skamarock et al., 2008; Jimenez et al., 2016b). They used two mass-flux type shallow convective parameterizations, namely those of Deng et al. (2003, 2014) and Grell and Freitas (2014). They found almost no seasonal bias from observations when the radiative effects at surface of both deep and shallow convection were taken into account for. Yet they did not study the cloud dynamics in the convective scheme.

Our aim is to extend on the previous studies to investigate the performance of convective parameterizations on a $\mathrm{ShCu}$ case with coupled radiation, turbulence and surface. To this end, we simulate a representative $\mathrm{ShCu}$ day with explicit coupling of the processes , i.e. LES, together with a process interaction represented by parameterized processes, i.e. mesoscale models. We pay special attention to key processes in the radiation-surface-cloud interactions. These are: first, the onset time, formation, intensity and spatial characteristics of the cloud population; and second, the spatio-temporal variability of shortwave radiation, including direct and diffuse partitioning. This is key to plant transpiration and, consequently, to surface flux regulation.

The originality of our research strategy relies on a unique chain of numerical experiments of a representative $\mathrm{ShCu}$ field including coupled surface and radiative transfer schemes. By combining LES and mesoscale simulations we also investigate the performance of our experiments at (a) sub-grid and (b) terra incognita (Wyngaard, 2004; Ching et al., 2014). To support our analysis we make use of two LES models: DALES (Heus et al., 2010) and WRF-Solar in LES mode (hereafter, WRF-LES). The use of two different LES models ensures the consistency of our reference experiment. Our case is based on a typical latesummer day in The Netherlands over homogeneous grasslands, with an initially clear sky and shallow convection developing before noon (Vilà-Guerau de Arellano et al., 2014; Pedruzo-Bagazgoitia et al., 2017). Keeping our experiments idealized and identical in initial and boundary conditions enables us to optimally control their performance.

Section 2.2 describes the models and parameterizations as well as the design of the experiments. The main results of our work are displayed in Section 2.3, with DALES results present only in Section 2.3.1. Our findings are discussed and placed in the context of literature in Section 2.4, where we also underline the relevance of our approach for related fields. The final comments and a summary of our findings are given in Section 2.5. 


\subsection{Methods}

\subsubsection{Simulating and representing}

We perform two sorts of experiments in this study: experiments explicitly resolving most of the turbulent and cloud motions, i.e., LES experiments; and experiments with coarser resolution and parameterized turbulence, hereafter called mesoscale experiments. Schalkwijk et al. (2015) showed that mesoscale simulations do not resolve any of the (turbulent) motions below the timescale of hours and, thus, rely solely on the parameterizations. Meanwhile, LES resolves most of the turbulence down to few minutes (Schalkwijk et al., 2015)[see Fig. 8 therein]. Terminologically, we will refer to LES explicit experiments as simulations given that they explicitly resolve most of the motions and turbulent eddies within the domain following first principles. On the other hand, we will use terms representation or modeling when addressing results of mesoscale experiments.

\subsubsection{WRF-Solar and the convective parameterizations}

All the mesoscale experiments are performed using the mesoscale model WRF-Solar. It is based on the WRF model (Skamarock et al. (2008)) with additional developments aimed at providing improved shortwave radiation-related information at surface (Jimenez et al., 2016b). Given the wide range of schemes and options in WRF-Solar, we will here focus briefly on the settings chosen for the present study. We use the Mellor-YamadaNakanishi-Niino (MYNN) scheme (Nakanishi and Niino, 2006) to parameterize boundary layer turbulence in the mesoscale experiments. The microphysics scheme used is (Thompson et al., 2008), which considers cloud and rain drops, graupel, ice and snow in addition to water vapor. WRF-Solar computes the cloud fraction using a sum of liquid water and ice mixing ratios and dependent on the relative humidity at each gridbox (Xu and Randall, 1996; Hong et al., 1998). Information on radiative transfer and land-surface model schemes is shown in Sect. 2.2.4.

Despite many schemes existing for deep convection, the parameterizations aiming at shallow convection are scarce. In our study we make use of two convective parameterizations: the one by Deng et al. (2014) was selected due to its original design addressed for shallow convection explicitly; the scheme by Grell and Freitas (2014) was selected because, although originally a deep convection scheme, there have been modifications to allow for shallow convection. In both parameterizations a smooth transition from shallow to deep convection is allowed.

The WRF parameterization for shallow convection by Deng et al. $(2003,2014)$ is a mass flux based parameterization also accounting for neutrally buoyant clouds (NBC). The triggering of the cloud is obtained by combining the explicitly resolved turbulent kinetic 
energy (TKE) and sub-grid turbulence obtained from the boundary layer scheme (MYNN, Nakanishi and Niino (2006)). The closure of this convection scheme transitions smoothly from a TKE closure on shallow convection, understood as updrafts up to $4 \mathrm{~km}$, to a convective available potential energy closure in deeper convection similar to Kain and Fritsch (1990). In contrast to other convective parameterizations, it uses prognostic equations for sub-grid cloud fraction, water and ice mixing ratios as variables that are passed on to the radiation scheme. It provides a correction for cloud fraction dependent on the relative humidity of the gridbox cloud-free fraction. The reasoning is that computed cloud fraction is usually only linked to the size of the cloudy updraft, neglecting the radiative effect of NBCs detrained from the updrafts. The source term in the prognostic equations for sub-grid cloud fraction, water and ice mixing ratios is a function of the rate of detrainment from the updrafts. The NBCs can dissipate through several physical processes, including evaporation at cloud edge due to horizontal turbulent mixing, vertical diffusion, precipitation, ice settling, and cloud-top entrainment instability. For a more in-depth description the reader is referred to Deng et al. (2003).

The other convective scheme used in this study is a mass-flux scale-aware stochastic convective parameterization (Grell and Freitas, 2014). It is the latest version of a parameterization originally developed in Grell (1993) and extended by Grell and Devenyi (2002) with new modifications in the originating conditions of updrafts and downdrafts, trigger functions, inclusion of tracer transport and the possibility to allow for aerosol interactions. It diagnoses sub-grid cloud ice and water content separately, but not sub-grid cloud fraction. Thus, cloud fraction is computed following ( $\mathrm{Xu}$ and Randall, 1996; Hong et al., 1998) with the additional sub-grid cloud and ice water content. The inclusion of Arakawa's approach (Arakawa et al., 2011) in the scheme ensures that the parameterization assumptions hold on the limits where the gridbox is fully cloudy or fully clear. This parameterizations has been successful in representing a smooth transition of convection along scales at horizontal resolutions ranging from 5 to $20 \mathrm{~km}$ (Freitas et al., 2017), or from 3 to $50 \mathrm{~km}$ (Fowler et al., 2016). For a further insight in the parameterization, the reader is referred to Grell and Devenyi (2002) and Grell and Freitas (2014).

\subsubsection{Explicit LES}

We perform the explicit experiments with two LES models, WRF-LES and DALES. The Dutch Atmospheric Large Eddy Simulation (DALES, Heus et al. (2010); Ouwersloot et al. (2016)) has its foundations in the work by Nieuwstadt and Brost (1986). The version used in this study is DALES 4.1 with improvements in the mechanistic vegetation sub-model within the land-surface scheme allowing for sensitivity to direct and diffuse shortwave radiation partition (Pedruzo-Bagazgoitia et al., 2017).

WRF-LES relies on the explicit mode of the Weather and Research Forecast model using 
the Smagorinsky closure (Smagorinsky, 1963) as sub-grid scheme. The advantage of using the WRF model both for explicit as well as parameterized numerical experiments is that it allows us to discard discrepancies due to different model architectures.

The DALES and WRF-LES experiments present two main differences: the land-surface model and the microphysics scheme. The former is described in Sect.2.22.2.4. On the latter, DALES uses an all-or-nothing microphysics scheme with $q_{l}=q_{\text {tot }}-q_{\text {sat }}$ if $q_{\text {tot }}>q_{\text {sat }}$ and $q_{l}=0$ otherwise where $q_{l}$ is the liquid water mixing ratio, $q_{t o t}$ the total water mixing ratio and $q_{\text {sat }}$ the saturation mixing ratio. DALES assumes a cloud fraction of 1 if $q_{l}>0$ and 0 otherwise at each gridbox. This scheme combination has been successfully used in previous studies (Siebesma et al., 2003; Vilà-Guerau de Arellano et al., 2014). WRF-LES uses the same new Thompson scheme and cloud fraction calculation method as WRFSolar.

\subsubsection{The coupled surface and radiative transfer schemes}

All simulations make use of the RRTMG radiation scheme (Iacono et al., 2008). This scheme calculates the 1-dimensional radiative fluxes for both longwave and shortwave, including direct and diffuse components, at every vertical level. The fluxes are calculated based on the local profiles of temperature, moisture and the standard profiles of the following compounds up to roughly 20km: carbon dioxide, ozone, methane, nitrous oxide, oxygen, nitrogen and the halocarbons. WRF and DALES present slightly different standard profiles, thus showing some disagreements in the radiation at the same height.

All the experiments in WRF-LES use the unified Noah land-surface model (Niu et al., 2011) with four soil layers. The experiment in DALES uses an interactive land-surface model responding to changing atmospheric conditions (van Heerwaarden et al., 2010) and includes the vegetation mechanistic model by Jacobs and de Bruin (1997) with further development on the vegetation sensitivity to direct and diffuse radiation (PedruzoBagazgoitia et al., 2017). Differences between surface schemes motivated different soil conditions in WRF-Solar or WRF-LES and DALES to obtain similar domain average surface fluxes. Differences between surface schemes motivated different soil conditions in WRF and DALES to obtain similar domain average surface fluxes on a clear day. The moisture and temperature of the four soil layers for each experiment are shown in Table 1 .

\subsubsection{Numerical setup and experiments}

The case under study is inspired on an adaptation by Vilà-Guerau de Arellano et al. (2014) of late September observations in The Netherlands (Casso-Torralba et al., 2008). It shows an initially clear sky with shallow convection onset before noon and driven by 
the moisture and buoyancy at the surface. Highest cloud tops reach up to $4000 \mathrm{~m}$. Our idealized study prescribes no large scale forcings nor horizontal wind, and all simulations use bi-periodic boundary conditions.

All numerical experiments are performed between 7 and 17 UTC without spin-up time. Regarding the latter, we prioritized the simultaneous start of all experiments at the expense of the experiment-dependent spin-up time. The timestepping of each experiment is shown in Table 2.1.

We perform 4 mesoscale experiments, the first three differing in the convective parameterization used: no parameterization is used in NOPAR $\_9$, the convective parameterization by (Deng et al., 2003) is used in DENG_9, and the GF_9 experiment uses the Grell-Freitas parameterization (Grell and Freitas, 2014). The motivation for the NOPAR_9 experiment is twofold: to assess if explicit convection at $9 \mathrm{~km}$ horizontal resolution provides realistic results, and to discriminate between the effects of the boundary layer parameterization and the convective schemes. The grid-spacing of these three mesoscale experiments is of $9 \mathrm{~km}$ in the horizontal, with 50 vertical levels distributed along $20 \mathrm{~km}$ following typical mesoscale operational settings: the lowest level is 20 meters thick, while at $5000 \mathrm{~m}$ high it is of about $400 \mathrm{~m}$. The domain size for these simulations is of $1800 \mathrm{x} 1800 \mathrm{~km}^{2}$. The last experiment, NOPAR_3, is identical to the NOPAR_9 experiment but with a 3 times finer grid-spacing in the horizontal, i.e., $3 \mathrm{~km}$. The domain size is of $600 \mathrm{x} 600 \mathrm{~km}^{2}$. This experiment aims at showing whether the resolved convection at $3 \mathrm{~km}$ is enough to better represent the shallow convection compared to the parameterized DENG9, GF9 and the explicit NOPAR_9 experiments.

The WRF-LES and DALES experiments use a horizontal and vertical grid-spacing of 50 meters and 12 meters, respectively, with a domain of $24 \times 24 \mathrm{~km}^{2}$. WRF-LES gradually stretches the vertical level thickness as it moves away from the surface, with a thickness

Table 2.1: Overview and relevant settings of the numerical experiments in the study.

\begin{tabular}{|c|c|c|c|c|c|c|c|}
\hline Experiment & Model & $\begin{array}{c}\text { Domain } \\
(\mathrm{X} \times \mathrm{Y} \times \mathrm{Z})\left(\mathrm{km}^{3}\right)\end{array}$ & $\begin{array}{l}\text { Gridpoint } \\
\text { numbers }\end{array}$ & $\begin{array}{l}\text { Time } \\
\text { step (s) }\end{array}$ & $\begin{array}{c}\text { Convective } \\
\text { parameterization }\end{array}$ & $\begin{array}{c}\text { Soil moisture } \\
\left(\mathrm{m}^{3} \mathrm{~m}^{-3}\right)\end{array}$ & $\begin{array}{c}\text { Soil temperature } \\
\text { (top to bottom) }(\mathrm{K})\end{array}$ \\
\hline DALES & DALES & $24 \times 24 \times 5.5$ & $480 \times 480 \times 456$ & $\begin{array}{c}\text { Adaptive } \\
\text { following } \mathrm{CFL}^{1}\end{array}$ & explicit & 0.385 & $282,282.5,283,284$ \\
\hline WRF-LES & $\begin{array}{l}\text { WRF-Solar } \\
\text { (LES mode) }\end{array}$ & $24 \times 24 \times 20$ & $480 \times 480 \times 200$ & 0.3 & explicit & 0.340 & $288,288.5,289,290$ \\
\hline WRF-LES_meso9 & $\begin{array}{l}\text { WRF-Solar } \\
\text { (LES mode) }\end{array}$ & $27 \times 27 \times 20$ & $3 \times 3 \times 50$ & 0.3 & explicit & 0.340 & $288,288.5,289,290$ \\
\hline NOPAR $\_3$ & WRF-Solar & $600 \times 600 \times 20$ & $200 \times 200 \times 50$ & 15 & explicit & 0.340 & $288,288.5,289,290$ \\
\hline NOPAR_9 & WRF-Solar & $1800 \times 1800 \times 20$ & $200 \times 200 \times 50$ & 50 & explicit & 0.340 & $288,288.5,289,290$ \\
\hline DENG_9 & WRF-Solar & $1800 \times 1800 \times 20$ & $200 \times 200 \times 50$ & 50 & Deng et al. (2003) & 0.340 & $288,288.5,289,290$ \\
\hline GF_9 & WRF-Solar & $1800 \times 1800 \times 20$ & $200 \times 200 \times 50$ & 50 & $\begin{array}{c}\text { Grell and } \\
\text { Freitas (2014) }\end{array}$ & 0.340 & $288,288.5,289,290$ \\
\hline
\end{tabular}

${ }^{1}$ Courant-Friedrichs-Lewy criterion: $\mathrm{CFL}=\max \left(\left|\frac{u_{i} \Delta t}{\Delta x_{i}}\right|\right)=1$ with $\Delta t$ the timestep in seconds and $u_{i}$ and $\Delta x_{i}$ the wind speed and grid-spacing in meters and meters per second, respectively, in the direction $i$ with $i=x, y, z$. 


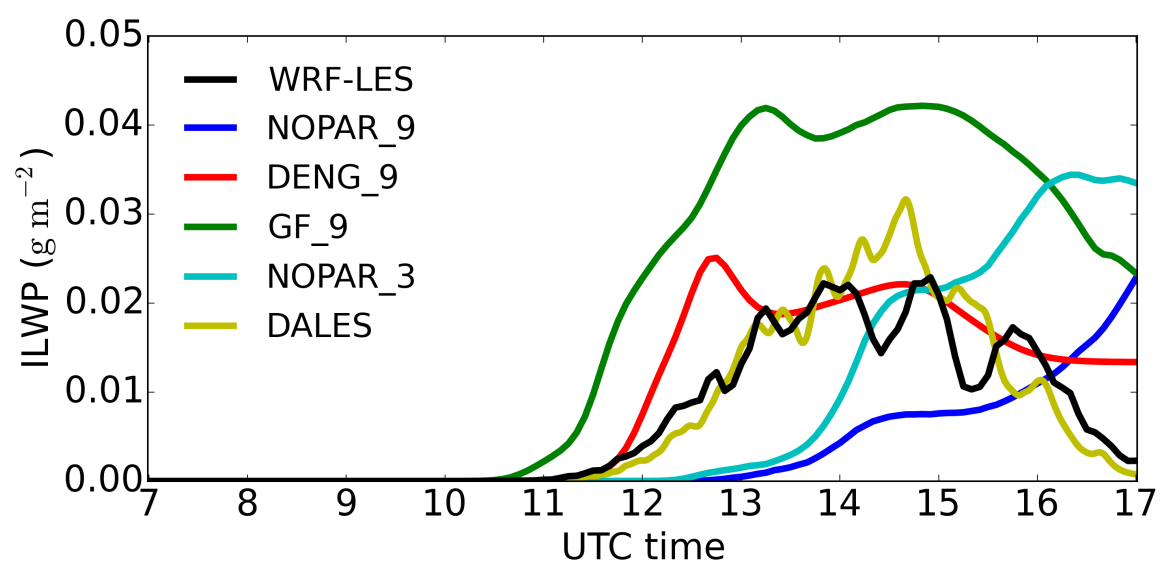

Figure 2.1: Temporal evolution of the domain averaged ice-liquid water path.

of about $60 \mathrm{~m}$ at the maximum cloud top height at around $5000 \mathrm{~m}$. The output of WRFLES is averaged horizontally over $9 \mathrm{~km}$ and vertically in WRF-LES_meso9 to match the mesoscale experiments' grid resolution. The horizontal averaging included a doublecounting of about $15 \%$ of the gridpoints to obtain a domain of $27 \times 27 \mathrm{~km}^{2}$. Thus the latter is not an experiment per se, but a re-griding of an explicit experiment to determine the role of filtering small scales and to mimic the mesoscale resolution. We acknowledge the limited domain in WRF-LES_meso9 and assume that, although not statistically robust, a comparison with resolution-equivalent mesoscale simulations is insightful. We show DALES and WRF-LES experiments for the first part of the study to show the robustness of the case and the good agreement between different LES models. We keep only WRFLES for the remaining part of the study to keep a as similar as possible model architecture between LES and mesoscale simulations. A brief overview on the experiments' settings and schemes is shown in Table 2.1.

\section{$2.3 \quad$ Results}

The development of ice-liquid water path (ILWP) over the whole domain is shown in Fig. 2.1. Both WRF-LES and DALES coincide on the onset of clouds happening at around 11 UTC, with a linear growth until 14 UTC and a further decrease starting after 15 UTC. We define the shallow convection period as the time between 11 and 15 UTC. Between 13 and 15 UTC the ILWP stabilizes around $0.022 \mathrm{~g} \mathrm{~m}^{-2}$ with discrepancies between the two explicit LES simulations due to the chaotic behavior of shallow clouds and to the different microphysics schemes.

A lack of convective parameterizations delays the onset of clouds both at 9 and $3 \mathrm{~km}$ resolutions. Furthermore, none of these 2 simulations predict a stabilization and decrease 


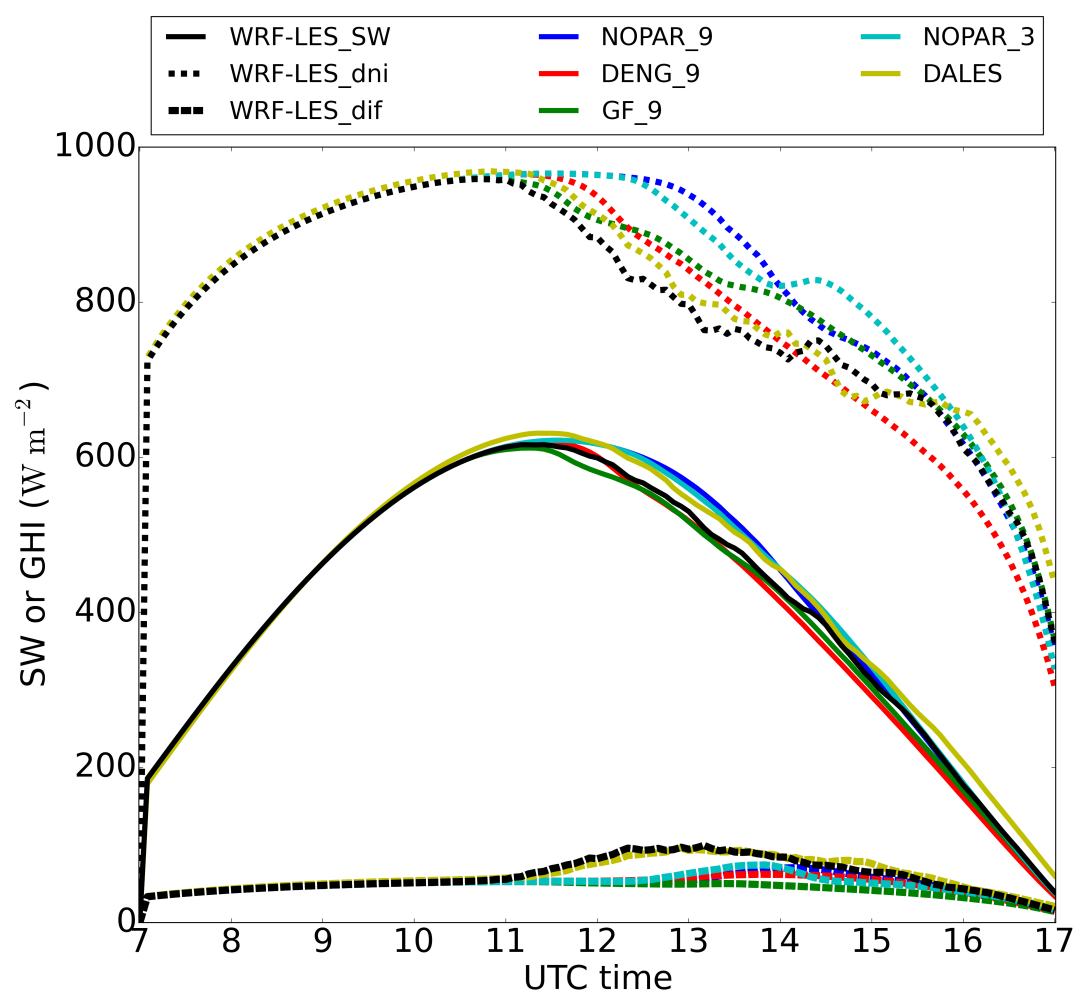

Figure 2.2: Downwards global horizontal (full), direct normal (dotted) and diffuse (dashed) irradiance at the surface for the three WRF mesoscale experiments, WRF-LES and DALES.

of ILWP. Instead, they show a delayed growth and a further increase even after 15 UTC. The DENG_9 experiment shows an improvement in cloud onset time, and on the overall evolution of ILWP during the day, with a stabilization of ILWP at 13 UTC and a (too little) decrease after 15 UTC. However, it predicts a too fast growth of ILWP between 12 and 13 UTC. GF_9 predicts a too early onset of the first clouds by about 30 mins, and, as with DENG_9, a too fast growth of ILWP during the early convection time reaching too high ILWP of up to $0.04 \mathrm{~g} \mathrm{~m}^{-2}$.

The domain averaged shortwave radiation (SW) at the surface (or global horizontal irradiance) and the normal direct and horizontal diffuse components are shown in Fig. 2.2. The first effects of clouds on global radiation appear at around 11:30 UTC according to WRF-LES and DALES. The small differences between WRF-LES and DALES before this time are due to discrepancies in domain top heights and in the profiles of chemical compounds to which the radiation scheme RRTMG is sensitive, as mentioned in Section 2.2 .

GF_9 shows a decrease already by 11 UTC while DENG gives a better timing for the first effect of clouds, consistent with Fig. 2.1. Afterwards, diffuse radiation peaks at 100 $\mathrm{W} \mathrm{m}^{-2}$ at around 13 UTC while direct and global irradiance decrease due to cloud shading. 


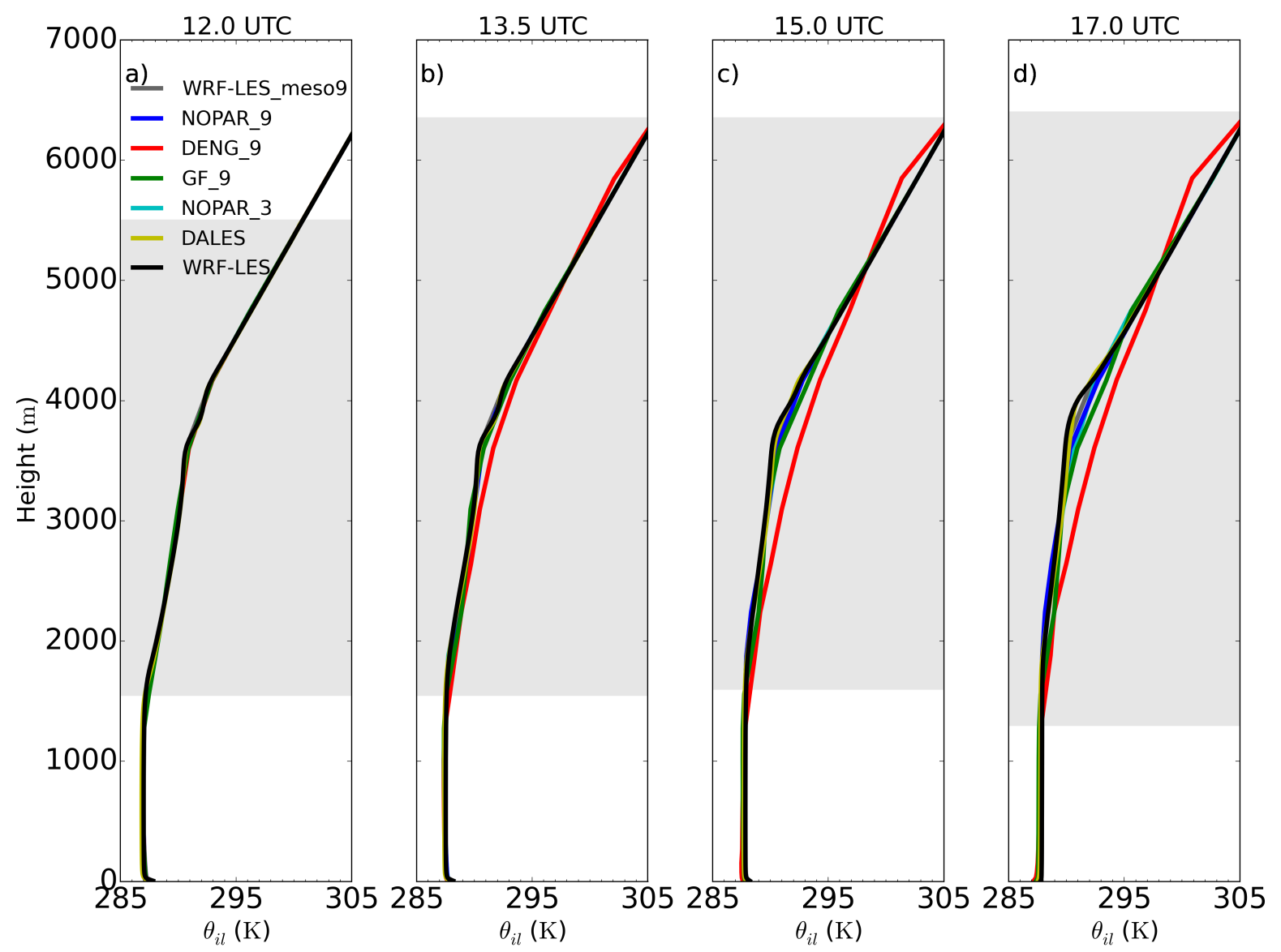

Figure 2.3: Vertical profiles of domain average ice-liquid water potential temperature $\theta_{i l}$ for the WRF mesoscale experiments, WRF-LES, DALES and WRF-LES_meso9 at four times along the numerical experiment. The gray shades show the cloudy region in at least one of the experiments.

After 13 UTC the agreement between WRF experiments in global radiation is better, with underestimations of up to $8 \%$, until the end of the shallow convection period. Due to the large ILWP overestimation by GF_9 shown in Fig. 2.1, this experiment underestimates the global radiation at the surface during the shallow convection period, specially in the 11-12 UTC period. There are important differences in the direct and diffuse contributions: only DALES and WRF-LES experiments predict a significant contribution of diffuse radiation at the surface $\left(\approx 100 \mathrm{~W} \mathrm{~m}^{-2}\right)$. The reason for this may be the large nonlinearities between cloud depth and diffuse radiation, specially for very shallow clouds that cannot be resolved at a mesoscale resolution. 


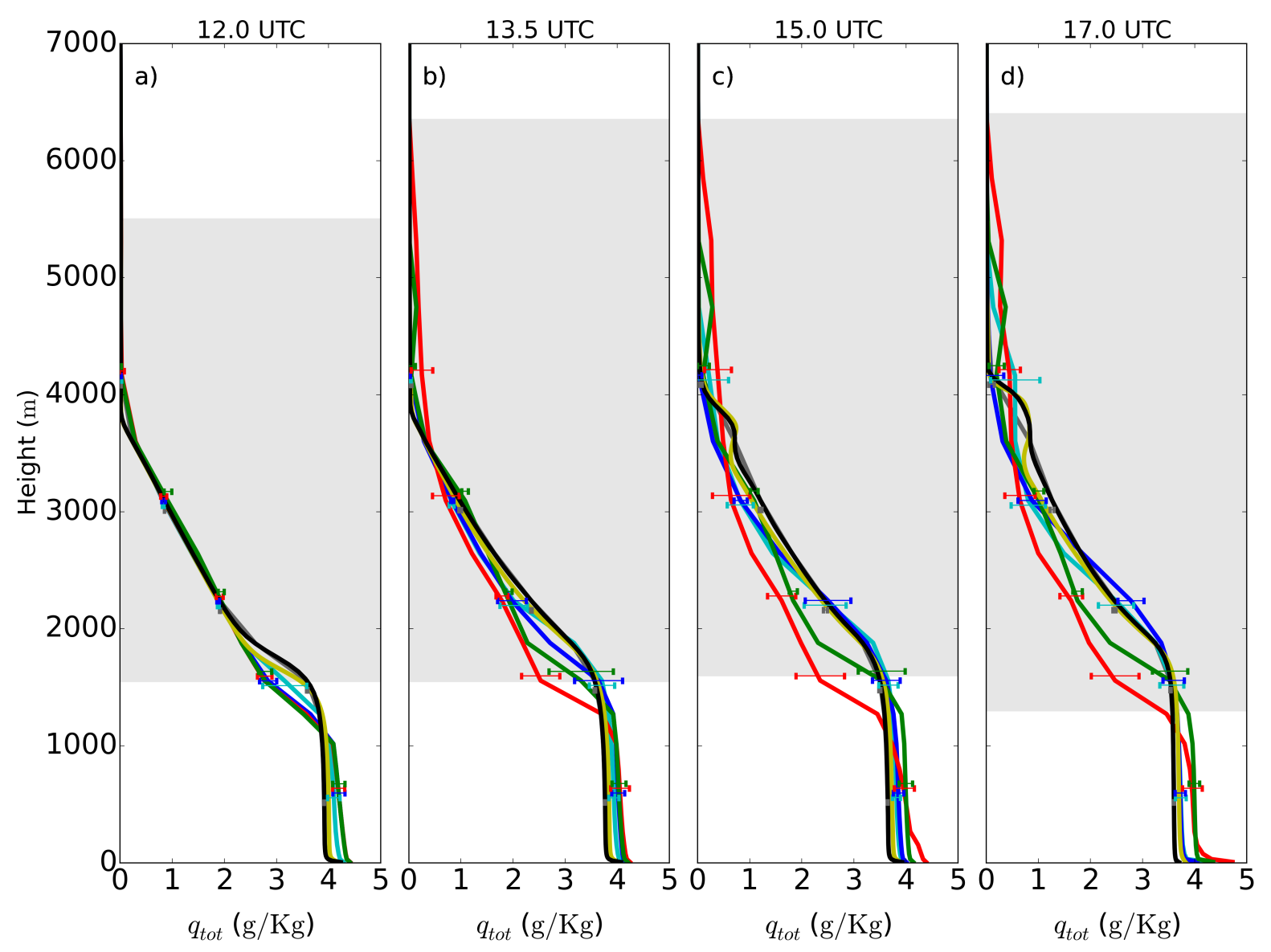

Figure 2.4: As in Fig. 2.3 but for total water mixing ratio $q_{t o t}$. Horizontal bars show the spatial standard deviation at selected heights for each experiment.

\subsubsection{Thermodynamic characterization}

Closely connected with the amount of available energy at the surface, we further explore the evolution of the atmospheric boundary layer (ABL) and cloud layer by showing the ice-liquid water potential temperature, $\theta_{i l}$, and total specific humidity, $q_{t o t}$, in Figures 2.3 and 2.4, respectively. The horizontal spatial variability within the domain is negligible in Fig. 2.3 and given by error bars at selected heights in Fig. 2.4. The results by DALES and WRF-LES experiments are consistent at all heights, with small discrepancies in boundary layer height and mixed layer values for $\theta_{i l}$ and $q_{t o t}$ of less than $0.2 \mathrm{~K}$ and $0.1 \mathrm{~g} \mathrm{Kg}^{-1}$ respectively, due to differences in surface fluxes during the morning. The $\theta_{i l}$ profiles of mesoscale experiments agree in the subcloud layer during the entire numerical experiment, and only until the cloud onset on layers above (not shown). There is a large spread among experiments, larger than within-experiment horizontal variability, in the moisture profiles below the cloud layer, indicating too little mixing in the boundary layer. This is also found in $\theta_{i l}$, although is less visible in Fig. 2.3. This finding is reinforced due to Bowen ratios of all WRF experiments differing by less than $5 \%$ (not 
shown). Such lack of mixing in the ABL is common in the MYNN scheme and leads to the underestimation of entrainment. Before cloud onset all mesoscale simulations underestimate (overestimate) Turbulent Kinetic Energy (TKE) in the upper (lower) half of the boundary layer by as much as $50 \%$ (not shown). NOPAR 3 improves the TKE representation within the ABL since the largest scales of TKE are now partly resolved (not shown). This improvement leads to a reduced entrainment underestimation on the mesoscale experiments, and consequently a reduction of the dry bias in the lower part of the cloud layer (not shown).

The atmosphere between 2000 and $5000 \mathrm{~m}$ shows a small but gradual cooling and moistening along the day in all experiments except for DENG_9 (Figs. 2.3 and 2.4). The moistening is a consequence of the rising moist updrafts. As a consequence of the observed underestimation in the boundary layer mixing, a drier layer above $1500 \mathrm{~m}$ and beyond intra-experiment variability is formed in the mesoscale experiments, being less severe in NOPAR_3. The cooling is driven by the longwave divergence caused by the water vapor gradient at that height (Fig. 2.4). The discrepancies in the magnitude of the cooling are partially explained by the effects of resolution (gray line in Fig. 2.3d at $3900 \mathrm{~m}$ ). Our reasoning is that at higher vertical resolution there is a sharper gradient in water vapor which, in turn, creates a more localized cooling than that of the mesoscale experiments with a smoothed gradient due to reduced vertical levels. The reduced cooling in GF_9 is due to higher moisture content at higher elevations, thus weakening the sharp initial gradient and leading to a lower impact of longwave flux divergence. The DENG_9 experiment particularly overestimates the ice-liquid water potential temperature between 2000 and $5000 \mathrm{~m}$ after $13 \mathrm{UTC}$, showing a $2 \mathrm{~K}$ warm bias at $3500 \mathrm{~m}$ by the end of the shallow convection period (15 UTC). This is partly due to the significant presence of moisture as high as $6000 \mathrm{~m}$ already at 13:30 UTC (Fig. 2.4b). Such high moisture content favours the early appearance of high clouds (Fig. 2.5a), which in turn causes the cooling by longwave divergence to happen at higher altitudes (almost $6000 \mathrm{~m}$ ). Moisture profiles of such a shape were already schematically described by Lenderink et al. (2004) as typical of schemes overestimating mass flux. These features can also be related to an underestimation on the entrainment inside the updrafts by DENG_9, as it will be shown in Fig. 2.7. Related to this, DENG_9 showed too large cloud updraft velocities after 12 UTC (not shown), which we hypothesize to be due to non-hydrostatic "pumping" (Deng et al., 2014). It is worth mentioning the accumulation of moisture after 15 UTC, clearer for explicit simulations at around $3900 \mathrm{~m}$ (Fig. 2.4c,d), due to the moist and buoyant updrafts from the surface reaching the more stable layer above this height (Fig. 2.3). This moisture accumulation is key in the growth of a second cloud layer at around that height.

Results of the cloud fraction vertical profile averaged over the whole domain are presented at Fig. 2.5 at three stages during the shallow convection period. They corroborate the DENG scheme leading to the formation of two cloud layers. Moreover, and comparing with 

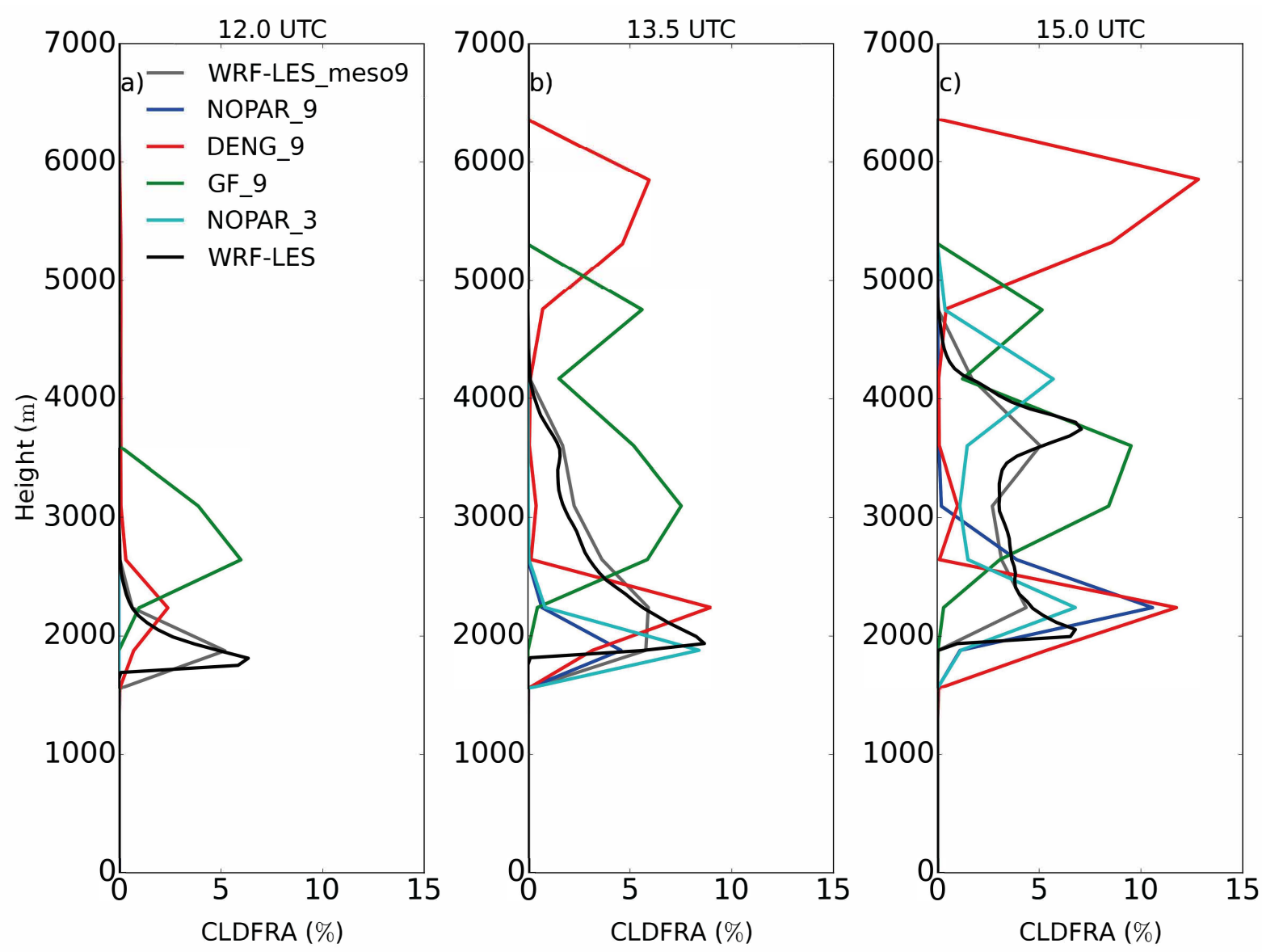

Figure 2.5: Vertical profiles of domain average cloud fraction at three times during the numerical experiment.

WRF-LES, we find that simulations at $9 \mathrm{~km}$ horizontal resolution are unable to represent the vertical growth of the cloud layer, regardless of whether clouds are parameterized or only resolved at mesoscale grid resolution. In particular, DENG_9 and GF_9 overestimate the superior cloud layer height and its growth rate (see Fig. 2.5b,c above $4000 \mathrm{~m}$ ), while NOPAR_9 under-represents and delays the vertical development of clouds. All mesoscale experiments show too much horizontal variability in cloud fraction, suggesting a non-homogeneously distributed cloud fraction along the domain (not shown). The delay in cloud onset in NOPAR_9 is explained by the need of complete saturation in a 9x9 km2 gridbox. We also observe that a fine vertical resolution is necessary to obtain a realistic time variability of cloud fraction. While in the LES experiments the cloud fraction varies in magnitude along the day (between $5 \%$ and $15 \%$ at $4000 \mathrm{~m}$ in from 15 to 16 UTC, not shown), the experiments with mesoscale resolution, including WRF-LES_meso9, are characterized by a much more constant cloud fraction vertical profile. Refining the horizontal resolution to $3 \mathrm{~km}$ without convection scheme (NOPAR_3) improves the cloud fraction profiles significantly, as it does capture the magnitude, although not the depth, of the shallow convection at 1330 UTC (Fig. 2.5b) and the two cloud layers at 15 UTC. Yet 

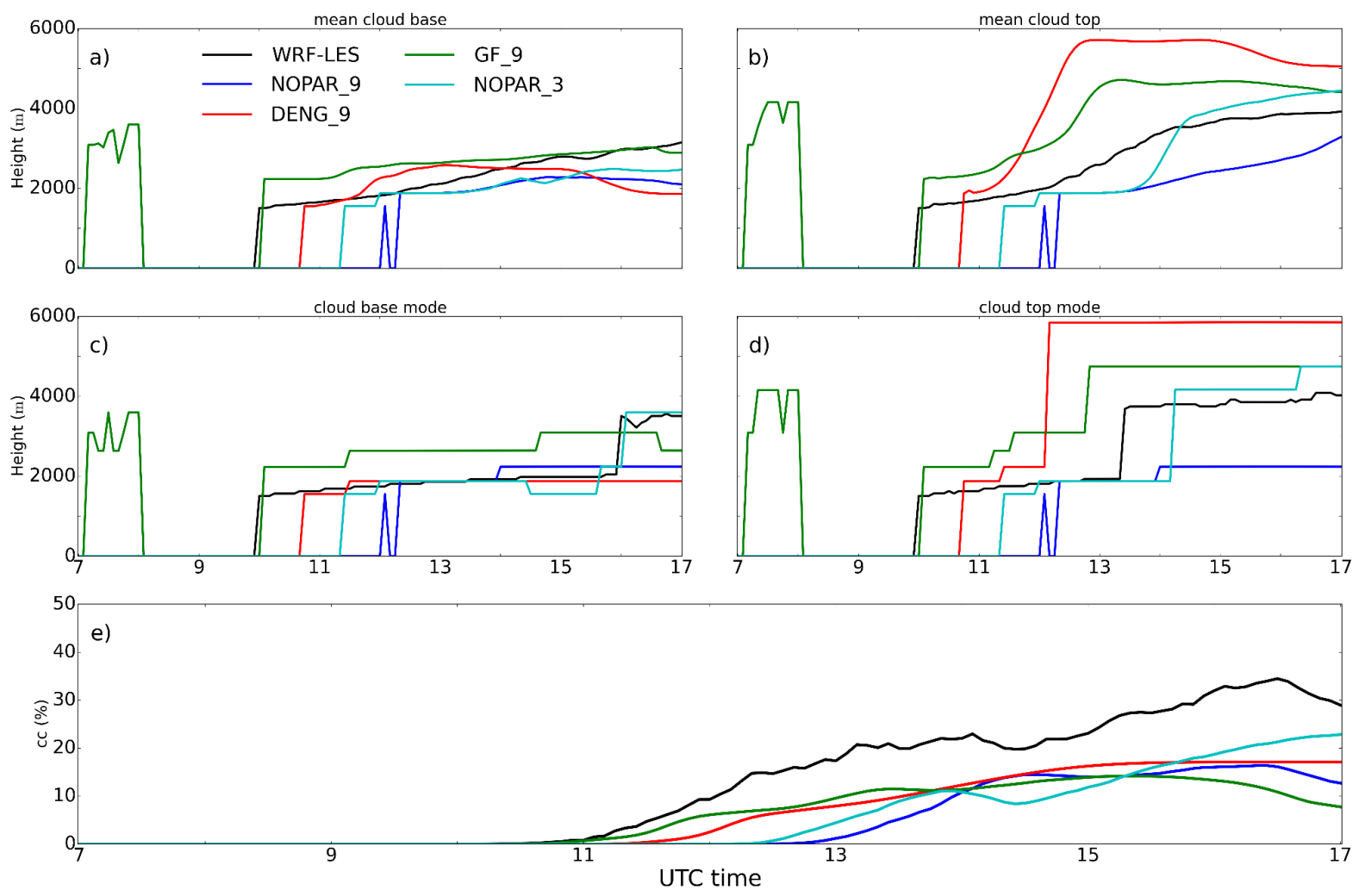

Figure 2.6: Temporal series of mean cloud base (a) and top (b), most frequent cloud base (c) and top (d) and cloud cover (e). The latter was calculated as the domain average of the column-integrated cloud fraction assuming maximum overlap.

the overestimation in the horizontal variability of cloud fraction by NOPAR 9 remains in NOPAR_3 (not shown).

\subsubsection{Cloud field evolution}

Focusing further on the cloud characteristics, a time series of mean and most frequent cloud base and cloud top is shown in Fig. 2.6. The first noticeable feature is the spurious clouds created by GF_9 in the first moments of the experiment. This, however, is not relevant in the development of the numerical experiment as it happens in a very reduced area (Fig. 2.6e). The sporadic presence of precipitation after 12 UTC may occasionally distort the cloud base calculations.

According to both of our LES experiments, first clouds appear at 10 UTC at around 1700 meters high. The base of most of the clouds follows the steady growth of the boundary layer along the day until about 16 UTC, when we observe a jump in the height of the most common cloud base up to about $3500 \mathrm{~m}$ (Fig. 2.6c). This height represents the second cloud layer previously visible in Fig. 2.5c. However, we deduce from the ascending mean 
cloud base (Fig.2.6a) that after 13 UTC there is already a significant number of clouds starting above 2000 meters high. We infer from the cloud top statistical mode that clouds remain very shallow until around 13:30 UTC. At that point, the more buoyant surface layer combined with the more moist environment due to previous updrafts enhances the growth of clouds up to $4000 \mathrm{~m}$. The continued longwave cooling and the increased moisture at those heights increases the persistence of clouds at $4000 \mathrm{~m}$, as suggested by the rising mean cloud top and the jump in cloud top mode between 13 and 14:30.

The mesoscale experiment GF_9 represents correctly the onset of the first clouds, although, as pointed out in Fig. 2.5a, it overestimates their height by at least $500 \mathrm{~m}$. This height overestimation is due to the convective parameterization, as NOPAR $\_9$ and DENG_9 give a better approximation to the initial cloud base and top heights. These two, however, miss the timing of the onset by almost one hour. Figures $2.6 \mathrm{~b}$ and $2.6 \mathrm{~d}$ agree with the performance of DENG_9 shown in Figs. 2.4 and 2.5: that an initial overestimation in moisture transport leads to an early and too high cloud layer which remains for the rest of the experiment at almost $6000 \mathrm{~m}$. Figure 2.6e shows the clear delay on onset and an underestimation of cloud cover (column-summed cloud fraction) by mesoscale simulations compared to the explicit experiment, missing almost $50 \%$ of the domain cloud fraction during the shallow convection period.

\subsubsection{Cloud and radiative spatial representation}

In order to study the dynamic evolution of clouds and their impact on shortwave radiation, we show in Fig. 2.7 the time evolution of ice-water content and downwards shortwave global radiation profiles for a representative column in each numerical experiment. We show a $9 \times 9 \mathrm{~km}^{2}$ domain averaged column for the WRF-LES and NOPAR_3 experiment for an area-equivalent comparison. The WRF-LES_meso9 experiment shows a growing cloud layer, starting after 10 UTC, with a rising cloud top which stabilizes at $5000 \mathrm{~m}$ after 13 UTC. The increased density of the cloud from 13 to 15 UTC between the 2000 to 4000 meters altitude represents the second cloud layer. This increased density reduces the global shortwave radiation at the surface to $400 \mathrm{~W} \mathrm{~m}^{-2}(60 \%$ of the value above the clouds) at 14 UTC. The spurious variations of cloud base after 13 UTC are due to precipitating water that evaporates before reaching the surface.

The figure corroborates the inability of all the mesoscale experiments to reproduce the cloud cycle gradually and as continuum. The NOPAR_9 experiment shows short lived, sporadic and relatively shallow but very dense clouds. Their effect in shortwave radiation is much more intense and sudden, to such an extent that the clouds at 15 UTC prevent almost any shortwave radiation reaching the surface. These very dense clouds are not very frequent in the domain, however, and as a consequence the average surface shortwave radiation is overestimated after 11 UTC (Fig. 2.2). The use of a convective 

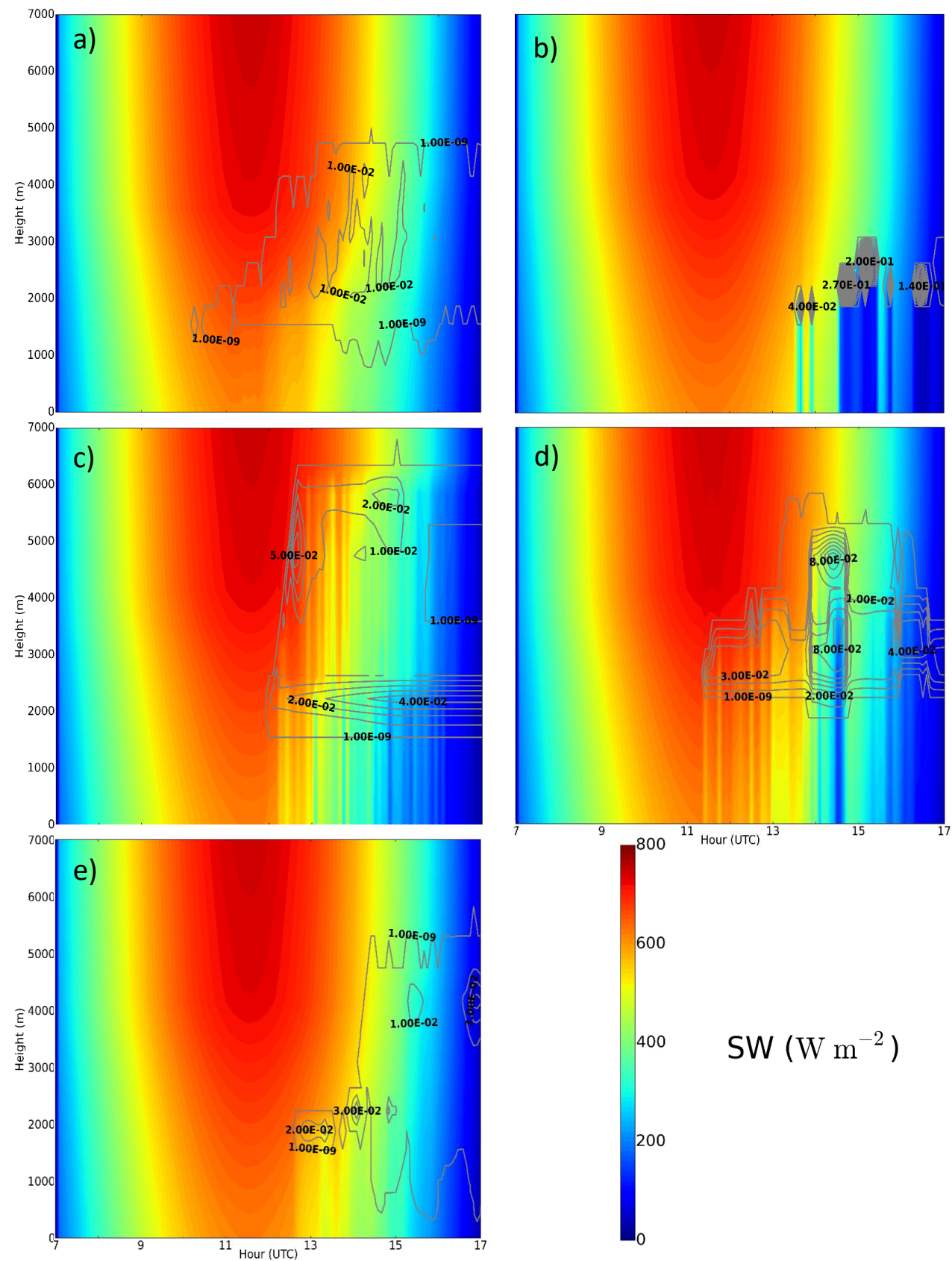

Figure 2.7: Time series of vertical profiles of selected representative gridpoints (or corresponding 9x9 $\mathrm{km}^{2}$ area) in WRF-LES_meso9 (a), NOPAR_9 (b), DENG_9 (c), GF_9 (d) and NOPAR_3 (e). Global downwards shortwave radiation is shown in shaded colours, and ice-liquid water mixing ratio in gray contour lines.

parameterization in the mesoscale experiments improves these results. The DENG_9 experiment shows less sudden and extreme variations in ice-water cloud content as well as on shortwave radiation compared to the NOPAR_9 experiment. However, it shows too high SW until 12 UTC due to the delay in cloud onset. The presence of the second cloud 
layer only 30 mins after the onset of first clouds conditions the shortwave radiation below $6000 \mathrm{~m}$ during the shallow convective period. This fast growth suggest, as stated before, an underestimation of the entrainment in the convective updrafts by the convective scheme. Such a dense second layer (up to $5 \mathrm{~g} \mathrm{~kg}^{-1}$ ), combined with a growing low layer, implies a too high variability on the surface shortwave radiation. The continued growth of the low layer after 1430 UTC explains the underestimation of shortwave radiation at the surface of as much as $150 \mathrm{~W} \mathrm{~m}^{-2}$ for this column, a feature also visible on the full domain average in Fig. 2.2. As shown in Fig 2.6 the timing of the first disturbances on SW is better captured by the GF_9 experiment. The rapid growth of the average water content in clouds (up to $8 \mathrm{~g} \mathrm{~kg}^{-1}$ in both layers), in turn, explains an overestimation of SW fluctuations during the shallow convective period similar to that of DENG_9. In this case, the larger extension (Fig. 2.5) and density (not shown) of the clouds between 14 and 15 UTC lead to overestimations of the SW reduction, also found in the domain average (Fig. 2.2). Finally, we show that a refined horizontal resolution of $3 \mathrm{~km}$ improves the shown results without the need of a convective parameterization. Fig. 2.7e shows a delayed onset of clouds and a growth up to $5000 \mathrm{~m}$ almost 2 hours after the onset of the first cloud, contrasting the immediate onset and growth by DENG_9 in Fig. 2.7c. Furthermore, values for ice-water cloud content, although overestimated, are closer to the ones suggested by WRF-LES_meso9 of the order of $0.01 \mathrm{~g} \mathrm{~kg}^{-1}$. Contour lines below 1500 $\mathrm{m}$ are due to precipitating water.

Our analysis turns now to study the impact of explicit and parameterized convection in the horizontal distributions. In Figs. 2.8 and 2.9 we show the ice-liquid water path over the domain for each experiment, and the instantaneous normalized shortwave radiation along the indicated dashed white lines, with horizontal direct and diffuse components in salmon-orange and yellow respectively. In addition, the histogram inset in the bottom left corner of each subfigure shows the distribution of ILWP. We selected 11:50 UTC as the plotting time given the compromise needed between a developed shallow cumulus field and a minimum impact of previous clouds on the experiment thermodynamics. We however show NOPAR_9 at 13:50 UTC as this experiment shows a clear delay in the onset of clouds (Fig.2.6e). Figure 2.8 shows WRF-LES and WRF-LES_meso9 experiments, while the three mesoscale experiments NOPAR_9, DENG_9 and GF_9 are contained in Fig. 2.9 .

The field in the explicit experiment WRF-LES shows a cloud cover of $8.14 \%$, and very high variability of ILWP among different columns with localized maxima of $350 \mathrm{~g} \mathrm{~m}^{-2}$. The shortwave radiation varies accordingly below the clouds, showing relevant differences in the partition of direct and diffuse SW. Under clouds with low ILWP the global SW reduction is limited, and the contributions of diffuse radiation is larger than that of direct radiation. In contrast, the global SW at the surface is purely diffuse and significantly reduced for denser clouds columns starting at values of ILWP around $10 \mathrm{~g} \mathrm{~m}^{-2}$. The ILWP histogram shows a gradual decrease towards higher values typical from shallow cumulus fields (Vogelmann 


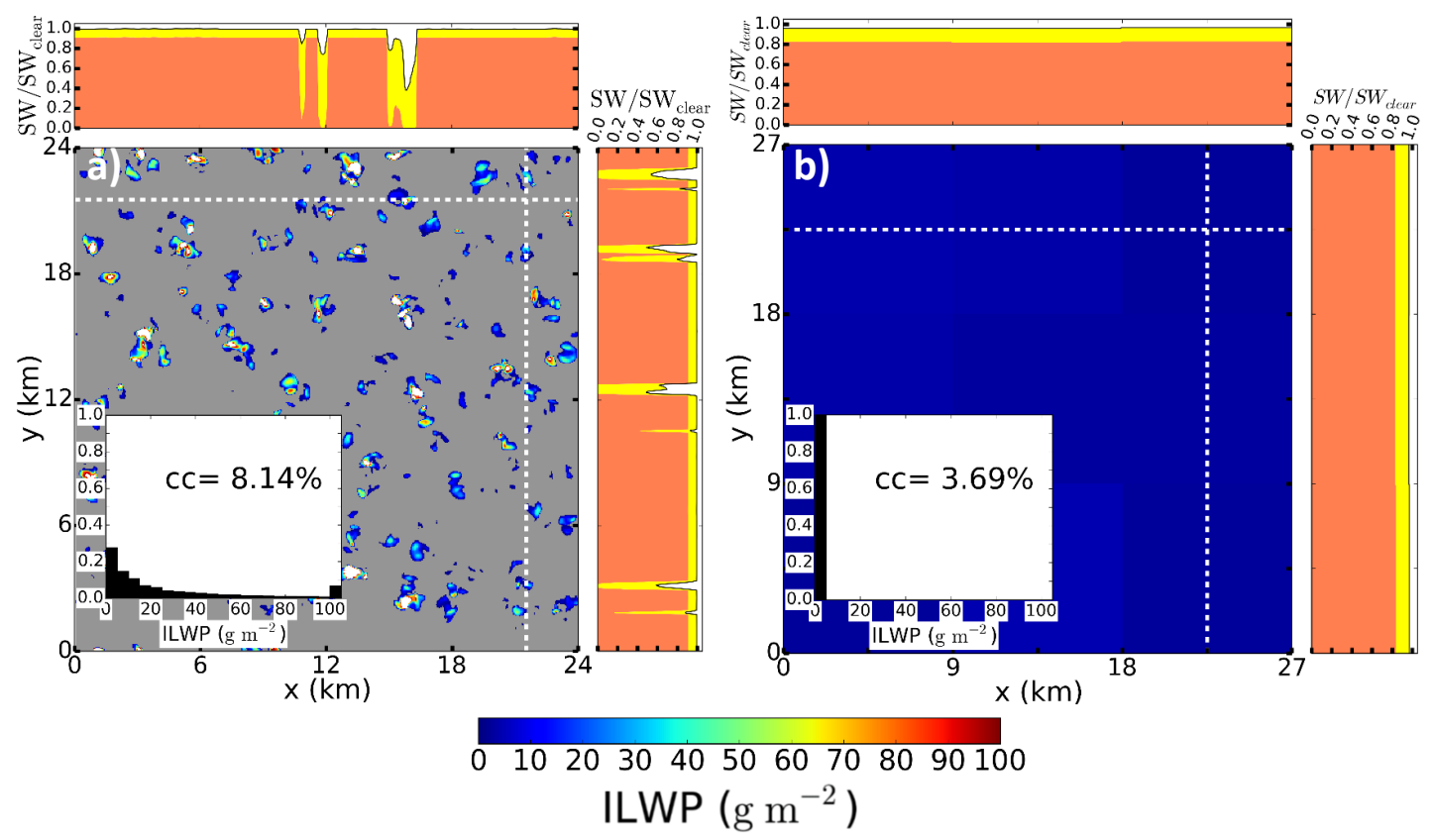

Figure 2.8: Instantaneous ILWP for WRF-LES (a) and WRF-LES_meso9 (b) at 11:50 UTC, where columns with ILWP $=0$ are displayed in gray. The horizontal and vertical side plots show the global (black line) shortwave radiation at the surface and the contributions of direct (salmon-orange) and diffuse (yellow) shortwave radiation. The inset at the bottom left of each subfigure gives the cloud cover (cc) and ILWP histogram at the shown time. Note that the rightmost bin includes all values above $100 \mathrm{~g} \mathrm{~m}^{-2}$, including any value above $110 \mathrm{~g} \mathrm{~m}^{-2}$ too.

et al., 2012). Note that the rightmost bin represents all columns with ILWP above 100 $\mathrm{g} \mathrm{m}^{-2}$ and that is not negligible as it accounts for nearly $8 \%$ of the total. Much of the heterogeneity in ILWP and SW is lost when adapting the results to a coarser mesoscale resolution in WRF-LES_meso9 showed in Fig. 2.8b. The mesoscale-averaged columns show a much more homogeneous field with low ILWP values between 0 and $5 \mathrm{~g} \mathrm{~m}^{-2}$ and a $3.69 \%$ cloud cover. Similarly, the strong local fluctuations of shortwave radiation, as well as the shift in direct and diffuse partition, are filtered out. The exponential-like decrease for larger ILWP present in the histogram in Fig. 2.8a shifts to a unique range of ILWP below $5 \mathrm{~g} \mathrm{~m}^{-2}$.

NOPAR_9 shows too high cloud cover, about 7.35\%, with highly heterogeneous values. The global shortwave radiation and its direct and diffuse components vary as in WRFLES, but at a much larger spatial scale $(9 \mathrm{~km})$ and thus overestimating the little spatial variability shown in WRF-LES_meso9. Furthermore, only under much deeper clouds direct radiation disappears. The DENG experiment shows a lower cloud cover to that of WRF-LES_meso9, as also shown in Fig. 2.6e. Yet we still find too many large ILWP values, as only $40 \%$ of the columns contain ILWP within the shallowest $0-5 \mathrm{~g} \mathrm{~m}^{-2}$ bin. As a consequence, we find highly varying global SW with too strong minima. The ratio of 


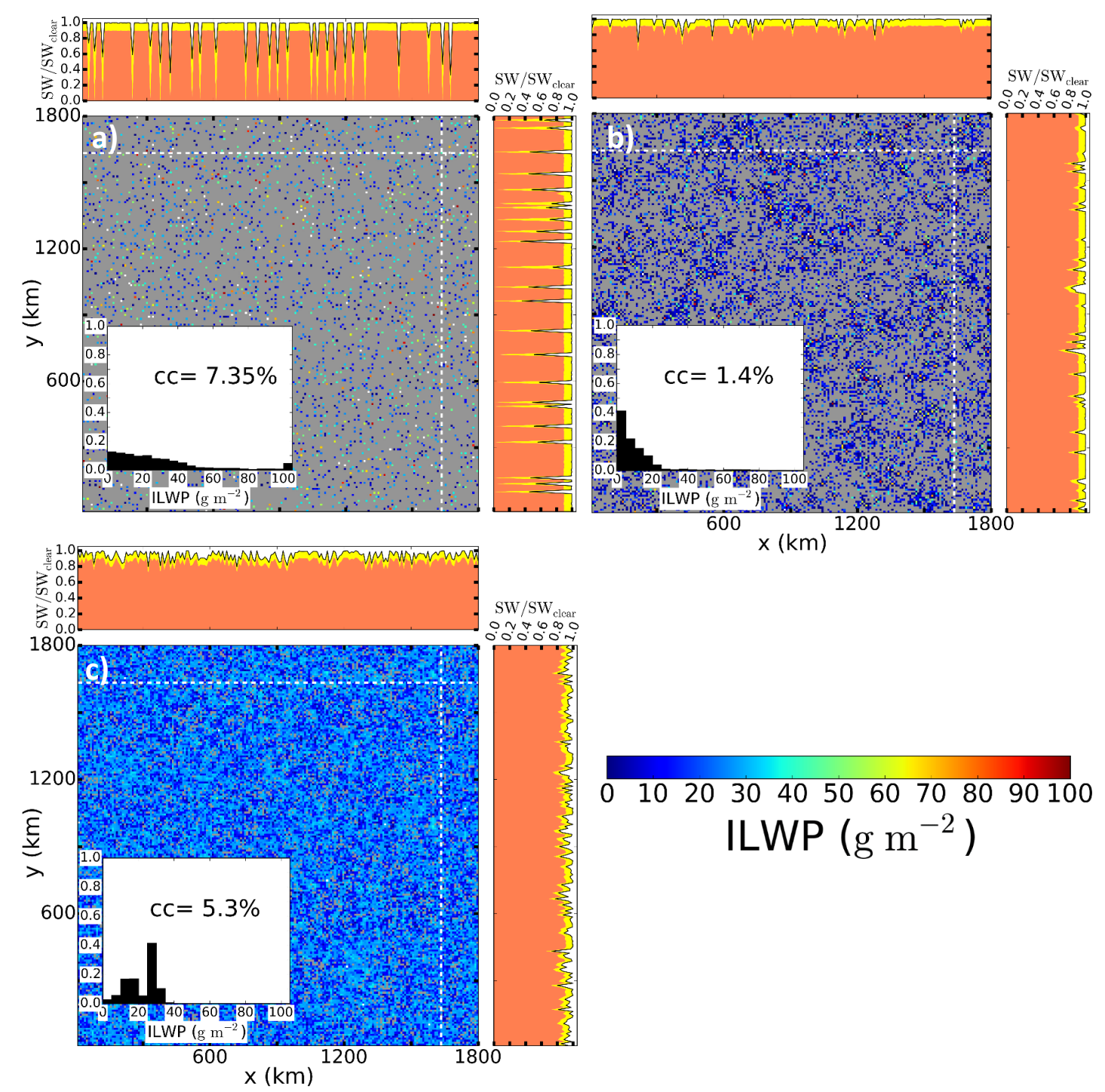

Figure 2.9: As in Fig. 2.8 for NOPAR_9 (a), DENG_9 (b) and GF_9 (c) experiments at 11:50 UTC, except for NOPAR_9 at 13:50 UTC.

direct and diffuse radiation does not change much along the domain, with an underestimation of diffuse radiation compared to Fig. 2.8b. The domain averaged underestimation is visible in Fig 2.2. GF_9 shows an overestimation of the cloud cover compared to WRF-LES_meso9. The spread of ILWP values in GF_9 is further increased, thus also overestimating the SW reduction due to clouds. GF_9 predicts on average more ice-water content than WRF-LES_meso9 and DENG_9, with more than 90\% of the cloudy gridboxes above the expected ILWP range of $0-5 \mathrm{~g} \mathrm{~m}^{-2}$ and a peak at $25-35 \mathrm{~g} \mathrm{~m}^{-2}$ bins. A plausible explanation for this may be the original purpose of the Grell-Freitas parameterization: the deep convection. Thus, the parameterization tends to more frequently generate larger and deeper column clouds than expected from WRF-LES_meso9, as it will be confirmed 


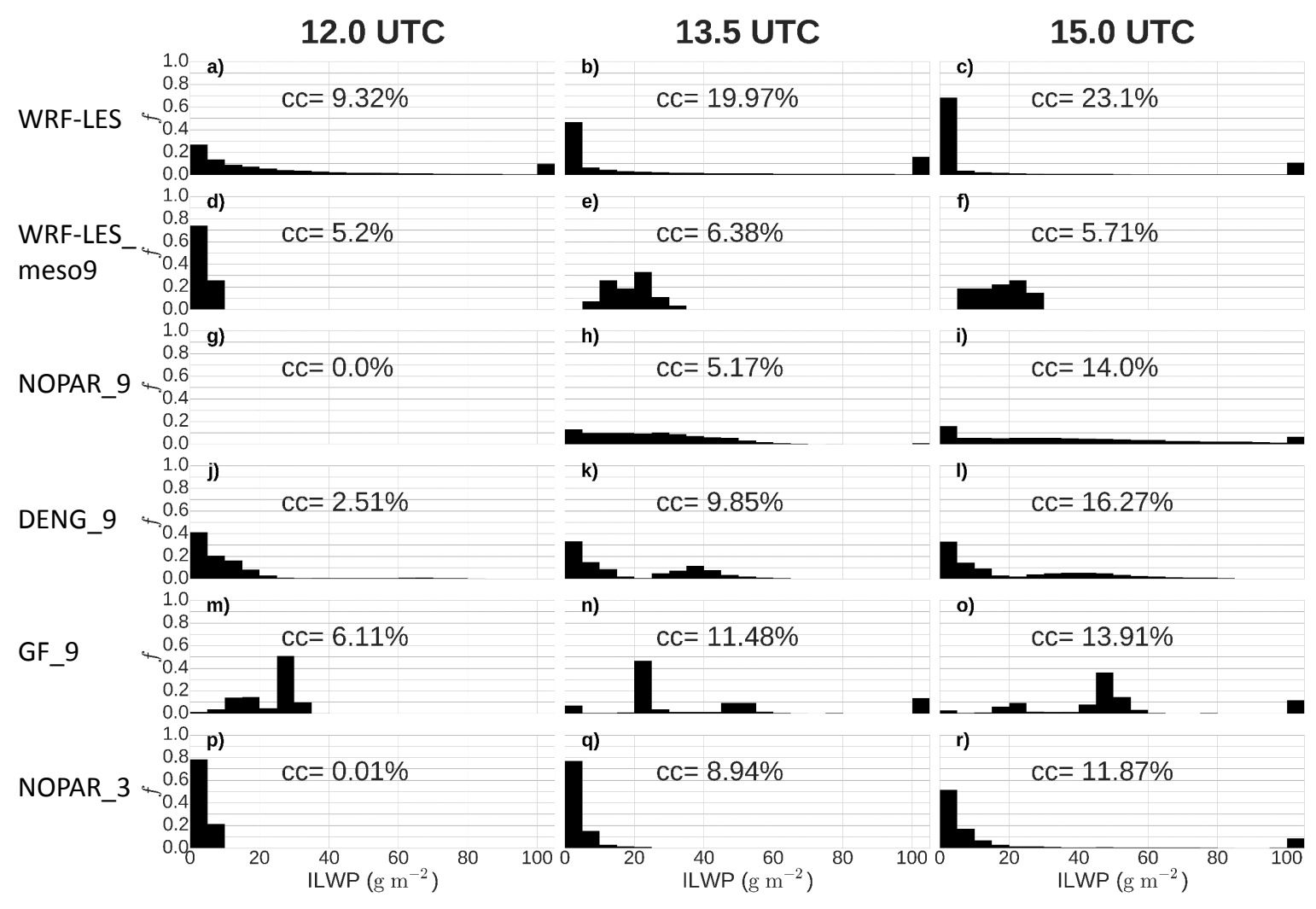

Figure 2.10: Cloud cover (cc) and histograms of ice-liquid water path frequency, $f$, for WRFLES (first row), WRF-LES_meso9 (second row), NOPAR_9 (third row), DENG_9 (fourth row), GF_9 (fifth row) and NOPAR_3 (sixth row) over a 15 minute interval centered in three selected times during the shallow convection: 12 UTC (left column), 1330 UTC (center) and 15 UTC (right).

in Fig. 2.10.

We show in Fig. 2.10 the distribution of ice-liquid water paths for our numerical experiments. WRF-LES shows less frequent columns for increasing ILWP, with more columns falling in the most shallow regime $\left(0-10 \mathrm{~g} \mathrm{~m}^{2}\right)$ as the experiment evolves (Figs. 2.10a,b,c). After removing the resolution effects, we observe most of the clouds to be very shallow (ILWP $<10 \mathrm{~g} \mathrm{~m}^{-2}$ ) at 12 UTC (Fig. 2.10d), and that starting at 1330 the cloud field stabilizes with most of the clouds falling between the 5 and $30 \mathrm{~g} \mathrm{~m}^{2}$ bins (Figs. $2.10 \mathrm{e}, \mathrm{f})$.

None of the parameterized experiments are able to represent adequately such a ILWP distribution. NOPAR_9, as first indicated in Fig. 2.9, shows a delayed cloud cover with too fast growth, and a very wide ILWP spectrum (Figs. 2.10h,i). DENG_9, although underestimating by a relative $50 \%$ the cloud cover at 12 UTC, shows an acceptable spectra with $60 \%$ of the cloudy columns falling within the expected 0-10 $\mathrm{g} \mathrm{m}^{2}$ bins. The evolution of the spectra in later hours is, however, very limited and far from the observed in WRF-LES_meso9. In fact, Figs. 2.10k,l suggest a bimodal distribution with maxima 
in the $0-5$ and $35-40 \mathrm{~g} \mathrm{~m}^{2}$ ranges. As mentioned in Fig. 2.9, GF_9 experiment shows here its preference for deeper convection. We find GF_9 to overestimate the most common ILWP values by 12 UTC already and to shift towards deeper values later on. Its bimodal distribution at 1330 and 15 UTC shows a too rigid preference by this scheme for either shallow convection, with ILWP between 20 and $30 \mathrm{~g} \mathrm{~m}^{-2}$, and the deeper mode with ILWP ranging from 40 to $60 \mathrm{~g} \mathrm{~m}^{-2}$. Finally, we find that the horizontal resolution refinement in NOPAR_3 is not enough to obtain a more similar spectra. Indeed, NOPAR_3 still shows too high cloud cover after a delayed onset, and a preference for too shallow clouds, showing that a $3 \mathrm{~km}$ horizontal resolution is not enough to account for most of the relevant shallow clouds.

\subsection{Discussion}

Lenderink et al. (2004) performed a thorough intercomparison of single column models for a shallow cumulus case, focusing on the turbulence, convection and condensation parameterizations. However, they prescribed both surface fluxes and prescribed radiative tendencies in the atmosphere. Brown et al. (2002) used radiative forcings from another model and prescribed surface fluxes for their turbulence-resolving LES intercomparison on shallow cumulus. Similarly, Siebesma et al. (2003) prescribed surface fluxes for their equilibrium shallow cumulus comparison in LES, and even neglected any cloud radiative effect in the radiation tendencies. In the comparison of explicit precipitating cumulus over sea by van Zanten et al. (2011) a net radiative forcing was prescribed throughout the atmosphere. By omitting the responses of the surface and the radiation to changes in the (parameterized for Lenderink et al. (2004), explicit for the rest) clouds, these studies miss the impact of potential interactions, such as surface dynamic heterogeneities by thick and thin cloud shading or dynamic effects of clouds, and the amplification or dampening of errors induced. Our approach with integrated and interacting cloud, radiation and surface schemes allows for a more realistic and integrated analysis. In fact, the excessive transport of moisture too high in the early phases of the shallow convection time (e.g. see Fig. 2.4b) impacts the development of the entire simulation by altering the radiation budget and thermodynamic profiles and, consequently, the growth of clouds. In particular, the experiment DENG_9 leads to warm and dry biases within the cloud layer of up to 2 $\mathrm{K}$ and more than $1 \mathrm{~g} \mathrm{~kg}^{-1}$ (about $25 \%$ of total humidity).

The possibility to solve explicitly the coupling between the radiation perturbations and the surface enables us to demonstrate the inability of the mesoscale experiments to reproduce either the spatial heterogeneities at the surface for global, direct and diffuse shortwave radiation (Figs. 2.8 and 2.9) or the domain average values (Fig. 2.2). The disagreement lies on an underestimation (overestimation) of diffuse (direct) shortwave radiation at surface by as much as $50 \%(10 \%)$ during most of the shallow convection period. Focused 
on the radiation effects, Jimenez et al. (2016b) showed that using one of the schemes studied here (Deng et al., 2003) lead to almost no bias for surface SW in the summer months. Our work, considering a more integrated approach, shows that an improvement on average surface SW does not guarantee a realistic representation of the boundary layer and cloud processes, raising the possibility of mutually canceling errors or compensating effects.

The need by convective parameterizations to account for shallow convection in everrefining resolution has been mentioned as one of the main challenges among the Numerical Weather Prediction models (Hong and Dudhia, 2012). Thus, the spatial characterization, and the sensitivity of current regional models to horizontal resolution is explored in our study in Section 2.3.3. As already pointed out in previous studies (Dudhia, 2014), the biases due to the convective parameterizations used in our study affect the thermodynamical state of the atmosphere and condition the further development of the experiments. This is improved when using no parameterization (NOPAR), at the expense of having a clearly non-realistic cloud cover (Fig. 2.6) and characteristics (Fig. 2.10). Refining the horizontal resolution to $3 \mathrm{~km}$ without convective scheme improves the representation for cloud fraction (Fig. 2.5) and cloud cover development (Fig. 2.7e), partly due to better mixing within the subcloud layer. Yet the average ILWP (Fig. 2.1) as well as its distribution (Fig. 2.10) shows large discrepancies with respect to a fully explicit LES experiment, by underestimating the presence of deeper clouds. In consequence, our study indicates that parameterizations are still needed for shallow convection as long as horizontal resolutions do not reach below $3 \mathrm{~km}$.

Finally, the results shown in this study are not only relevant for the regional numerical weather forecasting community, as the use of such a coupled model provides interesting outcome to several communities. In fact, the errors in shortwave radiation at the surface and, particularly, the misrepresentation of direct and diffuse radiation ratios by convective parameterizations, as well as their different spatial distributions are of critical relevance for solar energy forecast (Pedro and Coimbra, 2012). Direct and diffuse radiation partition is also of importance for the growing number of land-surface models sensitive to it, such as the DALES model used here or the land-surface model used by the Integrated Forecasting System by ECMWF (Boussetta et al., 2013). An adequate representation of direct and diffuse ratios is also necessary for accurate estimations on Gross Primary Productivity by land-surface models at all timescales (Alton et al., 2007; Cheng et al., 2015), and $\mathrm{CO}_{2}$ concentration and carbon cycle estimations (Mercado et al., 2009). In particular, the presence of diffuse radiation under certain conditions has been broadly linked to increased vegetation activity (Kanniah et al., 2012). Likewise, modeled isoprene biogenic emissions, known to be sensitive to direct-diffuse ratios (Guenther, 2013; Laffineur et al., 2013), may benefit from a more realistic representation such as the one shown in our explicit numerical experiments. This is also applicable to calculations of other chemical compound estimations (Madronich, 1987). 


\subsection{Conclusions}

Our research presents an integrated study on the effects exerted on cloud and boundary layer dynamics, radiation and surface by the performance of shallow convection parameterizations. Our novel methodology with coupled schemes allows to analyze the effects of convective parameterizations on the thermodynamics, radiation and surface of the simulation, as opposed to previous studies with prescribed surface or radiative fluxes. By keeping two different explicit LES model simulations (DALES and WRF-LES) as reference, we examine how convective schemes in WRF-Solar represent an idealized mid-latitude shallow convective summer day. We perform three simulations at the typical mesoscale operational resolution, i.e. $9 \mathrm{~km}$ in horizontal and 50 vertical levels up to $20 \mathrm{~km}$ : with no parameterized convection, and with two convective parameterizations by Deng et al. (2014) and Grell and Freitas (2014), respectively. An extra experiment at an intermediate horizontal resolution of $3 \mathrm{~km}$ is carried out without any convective scheme to explore the convection representation within the gray zone.

Coinciding vertical profiles of state variables and fluxes, time evolution and spatial distributions of clouds by both LES ensure the robustness of the case. We here summarize the findings, based on one representative but idealized case of shallow cumulus over the Netherlands, addressing different fields of interest:

- Interesting for land-surface model and solar energy harvesting, the domain average global horizontal irradiance at the surface is improved when using the convective parameterizations. However, the spatial (horizontal and vertical) variability and the direct and diffuse partition are not properly represented: they show too much variability and too little (much) diffuse (direct) radiation. See Figs. 2.2, 2.7, 2.8 and 2.9 .

- Related to the atmosphere thermodynamic structure, the parameterizations worsen the temperature and moisture profiles within the mixed boundary layer and cloud layer above: the cooling is too small in the cloud layer; there is too much drying, and too much moisture transported too high. See Figs. 2.3 and 2.4.

- On cloud characteristics, the timing of onset is improved by parameterizations. However, they predict too high cloud bases, too dense clouds and a too early and sudden creation of a too high second cloud layer. There is too much horizontal variability within the domain: The parameterizations tend to describe a cloud field with too deep clouds and with a too large domain averaged cloud cover. See Figs. $2.1,2.5,2.6,2.9$ and 2.10 .

The results of our study show that state of the art parameterizations for shallow convection are still not able to reproduce the essential characteristics that clouds exert on the surface-atmosphere system. Although they may predict variables such as domain av- 
erage shortwave radiation correctly, a broader analysis is needed to assess whether the schemes show the right values for the right reasons. Intermediate horizontal resolutions of $3 \mathrm{~km}$ proved to be not enough to reproduce the cloud features and effects by the fully explicit LES, as the smallest yet relevant scales of the shallow convection are not explicitly resolved. 


\section{Acknowledgments}

The first author would like to acknowledge the economic support by RAL and MMM departments for the stay at NCAR. This study was supported by the grant from the NWO ALW Open Programme (824.15.013). Computing resources (ark:/85065/d7wd3xhc) were provided by the Climate Simulation Laboratory at NCAR's Computational and Information Systems Laboratory, sponsored by the National Science Foundation and other agencies. 


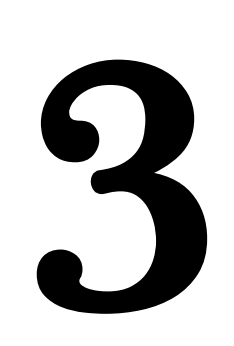

\section{Direct and diffuse radiation in the shallow cumulus-vegetation system: enhanced and decreased evapotranspiration regimes}

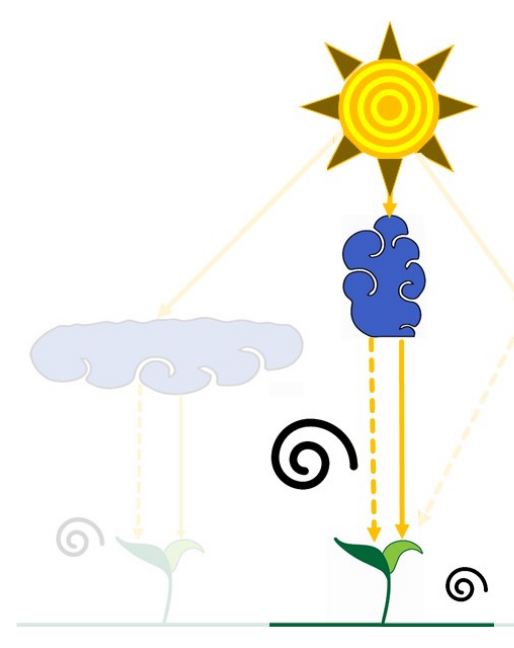

This chapter is published as:

Pedruzo-Bagazgoitia, X., Ouwersloot, H. G., Sikma, M., van Heerwaarden, C. C., Jacobs, C. M. J., and Vilà-Guerau de Arellano, J. (2017). Direct and diffuse radiation in the shallow cumulus-vegetation system: Enhanced and decreased evapotranspiration regimes. Journal of Hydrometeorology, 18(6):1731-1748 


\section{Abstract}

Guided by a holistic approach we investigate the combined effects of direct and diffuse radiation on the atmospheric boundary-layer dynamics over vegetated land on a daily scale. We design three numerical experiments aimed at disentangling the role of diffuse and direct radiation below shallow cumulus at the surface and on boundary-layer dynamics. We use a Large Eddy Simulation (LES) model coupled to a land-surface model, including a mechanistically immediate response of plants to radiation, temperature and water vapor deficit changes. We explicitly account for the partitioning in direct and diffuse radiation created by clouds and further inside the canopy. LES results are conditionally averaged as function of the cloud optical depth. Our findings show larger photosynthesis under thin clouds than under clear sky, due to an increase in diffuse radiation and a slight decrease in direct radiation. The reduced canopy resistance is the main driver for the enhanced carbon uptake by vegetation, while the carbon gradient and aerodynamic effects at the surface are secondary. Due to the coupling of $\mathrm{CO}_{2}$ and water vapor exchange through plant stomata, evapotranspiration is also enhanced under thin clouds, albeit to a lesser extent. This effect of diffuse radiation increases the water-use efficiency and evaporative fraction under clouds. The dynamic perturbations of the surface fluxes by clouds do not affect general boundary-layer or cloud characteristics due to the limited time and space where these perturbations occur. We conclude that an accurate radiation partitioning calculation is necessary to obtain reliable estimations on local surface processes. 


\subsection{Introduction}

The Atmospheric Boundary-Layer (ABL) dynamics are strongly influenced by the surfaceatmosphere exchange of heat and moisture, which, over vegetated land, is conditioned by $\mathrm{CO}_{2}$ concentration. Variations in atmospheric conditions due to turbulent motions locally modify surface fluxes at scales within a few hundreds of meters in the horizontal (Huang and Margulis, 2010), and modify the spatial average latent and sensible heat fluxes at regional scales (between 10 and $50 \mathrm{~km}$ ). These interactions are representative of the so called land-atmosphere feedbacks. As an illustrative example of the role of dynamics on such a large-scale coupling, de Bruin et al. (1989) already found that warm and dry entrained air was critical for sustaining high evaporation rates at the surface. van Heerwaarden et al. (2009) presented an overview on the factors within the ABL affecting evapotranspiration. The heterogeneities of the surface characteristics, or static heterogeneities, add to the complexity of land-atmosphere interactions by varying air temperature (Baldocchi and Ma, 2013) and the surface fluxes at a scale of several kilometers (Avissar and Schmidt, 1998; Esau and Lyons, 2002). At a regional scale, such surface heterogeneities have the potential to trigger secondary circulations (Garcia-Carreras et al., 2010).

A key factor that affects plant activity is the partitioning of radiation into direct and diffuse components, which depends on the transfer of radiation through the atmosphere, influenced by clouds and aerosols and through the canopy itself. Canopies convert direct to diffuse radiation by the scattering of light with leaves (Goudriaan, 1977; Norman, 1979). Aerosols and clouds are known to decrease total radiation and increase the diffuse fraction through light scattering. Barbaro et al. (2014) showed the relevant impact of aerosolradiation interactions on the boundary layer, but did not consider the effect that diffuse radiation would exert on the vegetated canopy. Diffuse radiation is known to increase the carbon (from here on representing only carbon from $\mathrm{CO}_{2}$ ) assimilation of vegetated canopies (Kanniah et al., 2012) due to a more homogeneous spread of radiation in the canopy, thus reducing the saturation of leaves at the top of the canopy and increasing the available radiation at the bottom and shaded parts of the canopy. Min and Wang (2008) found increased $\mathrm{CO}_{2}$ uptake by plants for conditions with atmospheric transmittance index below 1, which favor the scattering of radiation and promote diffuse radiation. An increase in carbon uptake over forests and under shallow cumulus clouds has been reported by several studies (Betts et al., 1999; Freedman et al., 2001; Oliphant et al., 2011) in spite of a reduction of global radiation. Since the vegetation-atmosphere system couples water and carbon cycles through photosynthesis, evapotranspiration and sensible heat flux exchanges are also affected by diffuse radiation (Wang et al., 2008; Oliveira et al., 2011).

Similar to aerosols, clouds also influence the partitioning between direct and diffuse radiation. However, the optical thickness of clouds can be larger than that for aerosols. Thick 
clouds absorb most direct radiation (Min, 2005), and little diffuse radiation reaches the surface. Below optically thin clouds the reduction in direct radiation can be significant but limited, and diffuse radiation is greatly enhanced (Cheng et al., 2016). We here follow the convention by (Min, 2005) where they defined thin clouds as the ones with cloud optical depth $\tau<8$, and thick clouds for larger $\tau$. The motivation was that clouds with $\tau>8$ did not allow any direct radiation go through. Similarly, Cheng et al. (2016) set the threshold at $\tau=7$. As we found in our study, however, such thresholds vary during the day, thus making the definition of thin and thick clouds quite arbitrary.

Convective clouds impose some additional effects on surface-atmosphere interactions compared to aerosols. Their onset, development and characteristics depend on surface conditions (Golaz et al., 2001; Garcia-Carreras et al., 2011; Chlond et al., 2014). They are known to vent air from the boundary layer to higher layers reducing the momentum, moisture and the subcloud-layer growth (Betts, 1973; Neggers et al., 2006), thereby altering the surface fluxes. More important to our study are the dynamic heterogeneities created at the surface by (inhomogeneous) cloud shading. As a result, clouds disrupt the surface energy balance by modifying surface temperature and specific humidity, creating inhomogeneities that vary the turbulent mixing near the surface. Lohou and Patton (2014) found a higher evaporative fraction on shaded surface because the latent heat flux $(L E)$ decreased less compared to the sensible heat flux $(S H)$. The shade-induced energy reduction at the surface promotes the narrowing of space between updrafts and, subsequently, the reduction of inter-cloud distance (Horn et al., 2015). Moreover, Gronemeier et al. (2016) found that cloud shading is able to generate secondary circulations, although its strength and significance depend on the solar zenith angles.

Most of these studies were idealized numerical experiments designed to disentangle the complexity of the cloud-surface interactions, and assumed free convective conditions with an instantaneous surface response. None of them, however, considered explicitly the impact of direct and diffuse radiation on active vegetation at the surface. This possibility poses interesting questions, such as whether the local impact of radiation partitioning by clouds on vegetation has a domain averaged effect, given the distinct responses for thin and thick clouds. Or, in addition, whether the direct-diffuse partitioning of radiation near the surface ultimately influences the characteristics of boundary-layer clouds themselves.

To our knowledge, no systematic study on the impact of shortwave direct and diffuse radiation, created both in clouds and within the canopy, on the boundary layer dynamics has been performed. Current developments in cloud observation (Schwartz et al., 2017) allow extensive studies on individual clouds, variability of cloud optical depth and solar irradiance within clouds and its effect on vegetation carbon uptake (Cheng et al., 2016). These advancements call for a similar procedure in explicit numerical simulations, where the impact of varying cloud optical depths at the surface are considered by performing systematic numerical experiments. The LES technique, with horizontal resolution in the 
order of 50 meters, has the capability to explicitly simulate shallow clouds and its spatial variability in cloud thickness (Vilà-Guerau de Arellano et al., 2014; Horn et al., 2015). A novel aspect of our LES study is the coupling to a land-surface model aware of both direct and diffuse radiation, leading to an integrated approach where diffuse radiation is explicitly treated both at the clouds and inside the canopy. The land-surface model uses a plant physiological scheme, including a mechanistic model for stomatal aperture, to account for very fast fluctuations (in the order of seconds) in diffuse and direct radiation and, thus, subsequent modifications on the carbon and water vapor exchanged between the vegetation and the atmosphere (Jacobs and de Bruin, 1997; Ronda et al., 2001). We perform systematic simulations where we use a radiative scheme to explicitly account for the direct and diffuse radiation generated by clouds, coupled to a canopy scheme where direct and diffuse radiation are treated explicitly. Such a high detail of $\mathrm{CO}_{2}$ processes at the surface also allows us to further investigate the relation between clouds and the carbon cycle (Vilà-Guerau de Arellano et al., 2012) at daily scales. With the high spatial resolution of our numerical experiments we are able to quantify the subgrid variability of larger scale models, e.g. Integrated Forecasting System (IFS), with similar mechanistic formulations for photosynthesis and stomatal conductance (Boussetta et al., 2013). The domain, in the order of $24 \times 24 \mathrm{~km}^{2}$ is representative of a typical grid box for regional carbon-climate models.

Aiming to shed light on the issues posed above, the research questions of this study are the following:

- Does direct and diffuse radiation modulation by shallow cumulus affect evapotranspiration and $\mathrm{CO}_{2}$ assimilation locally? And over the whole domain? How does this impact the partitioning of sensible and latent heat flux?

- Under these non-stationary surface conditions, do the direct and diffuse radiation alterations from clouds feed back into boundary-layer dynamics? Do cloud characteristics depend on it too?

The remaining part of this study is structured as follows: Section 3.2 describes the methodology and tools used for this study, including the implementation of a canopy radiative transfer scheme accounting for direct and diffuse radiation, as well as the research strategy. Section 3.3 contains the main findings and points of discussion of our analysis. Finally, the concluding summary of the research and recommendations are provided in Sect. 3.4. 


\subsection{Methods and numerical experiments}

\subsubsection{Explicit simulation of the coupling: DALES}

The Dutch Atmospheric Large Eddy Simulation (DALES) is under continuous development since the 80s (Nieuwstadt and Brost, 1986; Heus et al., 2010; Böing et al., 2012; Ouwersloot et al., 2016). It incorporates an interactive land-surface scheme responding to atmospheric processes described in Jacobs and de Bruin (1997), van Heerwaarden et al. (2010) and Vilà-Guerau de Arellano et al. (2015). Within the land-surface scheme, we use the plant physiological model A-gs (Jacobs and de Bruin, 1997) to simulate the behavior of vegetation and assuming an instantaneous response of vegetation to atmospheric and radiation forcings. The version used in this study is DALES 4.1 with additional upgrades concerning the radiation transfer within the canopy (Sect. 3.2.3.2) and conditional sampling at the surface (Sect. 3.2.4). We present here the most relevant parts of the land-surface model, that is, the contribution of vegetation to latent heat flux, the sensible heat flux and the carbon assimilation rate by vegetation.

The contribution of vegetation to total latent heat flux is given by:

$$
L E_{v e g}=c_{v e g} \frac{\rho L_{v}}{r_{a}+r_{v e g}}\left(q_{s a t}\left(T_{s}\right)-q\right)
$$

where $c_{v e g}$ is the vegetation cover fraction, $\rho$ stands for the air density and $L_{v}$ is the specific latent heat constant for evaporation. The specific and saturated specific humidity is given by $q$ and $q_{\text {sat }}$ respectively, and the surface temperature by $T_{s}$. The vegetation canopy resistance $r_{v e g}$, giving the capacity of stomata (upscaled at canopy level) to exchange water vapor with the environment, is obtained by $r_{v e g}=\frac{1}{g_{c}}$, where $g_{c}$ represents the stomatal conductance at canopy level. Further information on its calculation is given in Sect. 3.2.3.2 and in the supplementary material of this thesis. Since the vegetation cover is $90 \%$, leaving only $10 \%$ for bare soil, $L E_{v e g}$ is the main contributing term to the total latent heat flux, $L E$.

Finally, the sensible heat flux is calculated by:

$$
S H=\frac{\rho c_{p}}{r_{a}}\left(\theta_{s}-\theta_{\text {air }}\right)
$$

where $c_{p}$ is the specific heat capacity of air at constant pressure, $\theta_{s}$ is the potential temperature of the surface, and $\theta_{\text {air }}$ the potential temperature of the first level above the surface.

$A_{n}$, or the carbon assimilated through photosynthesis by the vegetation-canopy per sec- 
ond, is related to the vegetation resistance by:

$$
A_{n}=\frac{C_{s}-C_{i}}{r_{a}+r_{v e g_{\mathrm{CO}_{2}}}}
$$

where $C_{s}$ and $C_{i}$ are the external and internal $\mathrm{CO}_{2}$ concentrations respectively, and $r_{a}$ and $r_{v e g_{\mathrm{CO}_{2}}}$ the aerodynamic and vegetation canopy resistance for $\mathrm{CO}_{2}$ exchange, respectively, where $r_{v e g_{\mathrm{CO}_{2}}}=1.6 r_{v e g}$. The factor of 1.6 accounts for the different molecular diffusivity of water vapor and $\mathrm{CO}_{2}$ in the air (Jacobs and de Bruin, 1997).

For a detailed description on the treatment of direct and diffuse radiation in the canopy resistance calculations, the reader is referred to the supplemental material.

\subsubsection{Conceptual analysis: CLASS}

To support the analysis of the results in DALES, in Sect. 3.3.1.2 we make use of the Chemistry Land-surface Atmosphere Soil Slab model (CLASS) (Vilà-Guerau de Arellano et al., 2015). This box-model is based on the mixed layer equations to obtain the temporal evolution of the boundary layer and surface processes. The land-surface model in CLASS (van Heerwaarden et al., 2010) is similar to that of DALES and the schemes described in Sects. 3.2.3.1 and 3.2.3.2 are also present and used in CLASS. With this box model we analyse the response of vegetation to temporal changes in cloud optical depth and radiation in a more controlled environment than in DALES, where explicit turbulent makes interpretation difficult. By using CLASS, it is easier to fix the surface conditions and untangle the effects of different factors affecting carbon assimilation. We prescribe the onset, duration and disappearance of a cloud in CLASS by prescribing the cloud optical depth for each timestep.

\subsubsection{Direct and diffuse radiation partitioning}

In order to account for shortwave direct and diffuse radiation we first divide the atmosphere in two layers, separated at a height of $500 \mathrm{hPa}$. Above this level we consider a non-polluted standard Rayleigh atmosphere (Barbaro et al., 2014). We assume Rayleigh scattering to be the dominant scattering process due to the molecules present at these altitudes. Since our goal is to understand the explicit effect of direct and diffuse radiation generated by shallow cumulus, we assume all radiation at the top of the domain to be direct unless stated otherwise in the experiment. By doing this, we isolate the added contribution of cloud-generated diffuse radiation at the top of the canopy. We prescribe a maximum value of $S W_{d i r}^{T o D}=1000 \mathrm{~W} \mathrm{~m}^{-2}$ (unless stated otherwise) as a top boundary condition in the domain, which is a representative value for the downwards shortwave radiation at the height of $500 \mathrm{hPa}$ (Barbaro, 2015). To confirm the validity of the results, we design an additional experiment (AER, Sect. 3.2.5) with a combination of direct and 
diffuse radiation at the top of the domain. We explicitly simulate the shortwave radiative transfer between the $500 \mathrm{hPa}$ level and the surface. Concerning longwave radiation, only the upward and downward components at the surface are calculated, according to:

$$
\begin{gathered}
L W^{\uparrow}=\epsilon_{s} \sigma \theta_{s}^{4} \\
L W^{\downarrow}=\epsilon_{a i r} \sigma \theta_{\text {air }}^{4}
\end{gathered}
$$

where the emissivities for surface and air are $\epsilon_{s}=1$ and $\epsilon_{\text {air }}=0.8$ respectively, and $\sigma$ is Boltzmann's constant. This is necessary to obtain realistic radiation and surface balances at the surface. Thus, we do not account for longwave radiation from clouds or different gas concentrations, as we focus on the radiative transfer of shortwave radiation and do not expect significant differences in the longwave components between experiments.

\subsubsection{Radiative transfer in clouds: Delta Eddington approximation}

The Eddington method for shortwave radiative transfer was originally proposed by Shettle and Weinman (1970), and later approximated and further developed including a delta function by Joseph et al. (1976). This method has been already expanded for aerosols and successfully tested in DALES by Barbaro et al. (2014).

The delta-Eddington calculates the transfer of radiation with the following governing equations:

$$
\begin{gathered}
S W_{d i f}^{\uparrow}=I_{0}\left(\tau^{\prime}\right)-\frac{2}{3} I_{1}\left(\tau^{\prime}\right) \\
S W_{d i f}^{\downarrow}=I_{0}\left(\tau^{\prime}\right)+\frac{2}{3} I_{1}\left(\tau^{\prime}\right) \\
S W_{d i r}^{\downarrow}=\mu F_{0} e^{-\tau^{\prime} / \mu}
\end{gathered}
$$

where $\tau^{\prime}$ refers to the optical (cloud) depth after applying the delta-Eddington approximation that assumes most of the scattering to be forward. $S W$ stands for (upwards, $\uparrow$, or downwards, $\downarrow$, and direct, dir, or diffuse, dif) shortwave radiation, $\mu=\cos \theta$ where $\theta$ is the zenith angle and $F_{0}$ is the solar radiative flux perpendicular to the incidence direction at the top of the domain. The Eddington assumption supposes that we can decompose the total diffuse radiation $I_{d i f}$ as a superposition of two functions $I_{0}$ and $I_{1}$, such that they fulfill $I_{d i f}(\tau)=I_{0}(\tau)+\mu I_{1}(\tau)$ (Shettle and Weinman, 1970). The shape of $I_{0}$ and $I_{1}$ for different cases is given in Shettle and Weinman (1970). It must be noted that the delta-Eddington method is sensitive to the solar angle, as shown in Fig. 3.1. Here we assume the shadow of a cloud to be always right below the cloud, although we account for the solar angle dependences in the Delta-Eddington calculations and in the radiation intensity at the top of the domain. This is a reasonable approximation according to Schumann et al. (2002), who found shadow-asymmetries due to non-zero solar zenith angle to be irrelevant for turbulent motions in the boundary layer. We fix the effective radius 
of droplets to be $r_{\text {eff }}=0.01 \mathrm{~mm}$, a typical droplet size for shallow cumulus (Baker and Latham, 1979). This is relevant for obtaining the cloud optical depth based on the liquid water content, where we follow Stephens (1984). We performed a sensitivity analysis on the impact of the droplet effective radius on shortwave radiation and found little impact within the range typical for cumulus clouds (Lu et al., 2013) (results not shown).

For a complete explanation and derivation of the Delta-Eddington method, the reader is referred to Shettle and Weinman (1970) for the original Eddington method, to Joseph et al. (1976) for the delta-Eddington approximation and to Heus et al. (2010) and Barbaro et al. (2014) for its application.

\subsubsection{Radiative transfer in canopy: Gaussian method}

The limitation of single-big leaf models, like applied by Vilà-Guerau de Arellano et al. (2014), to account for canopy radiative transfer is tackled by explicitly calculating radiation profiles for direct and diffuse radiation. This is especially relevant for the current work, where both diffuse and direct radiation components are accurately calculated at the top of the canopy. To this end, we employ the scheme used by Jacobs and de Bruin (1997) and inspired from the radiative transfer approximations used in Goudriaan (1977) and adapted by Spitters (1986). A detailed description of the canopy radiative transfer and canopy upscaling is given in the supplemental material. In short, the main characteristics of the scheme are the following:

- Diffuse radiation has a (constant) extinction coefficient inside the canopy that is smaller (for most of the day) than that of the (solar angle-dependent) direct radiation.

- The direct radiation that is not transmitted nor absorbed is scattered by leaves or the ground and converted into diffuse radiation inside the canopy.

- Leaves are distributed spherically in the canopy, and affect the radiation penetration accordingly.

Total stomatal conductance is obtained at three levels inside the canopy after calculating the radiation profiles and the amount of shaded and sunlit leaves per level. For the calculations of stomatal conductance at leaf level we use the A-gs model described by Jacobs et al. (1996) and Jacobs and de Bruin (1997). Bulk stomatal conductance of $\mathrm{CO}_{2}$ for the canopy $g_{c}$ is obtained through the Gaussian integration method described in Goudriaan (1986) and implemented in the IFS model by the European Centre for Medium-Range Weather Forecasts, ECMWF (Boussetta et al., 2013). As in other studies (Jacobs and de Bruin, 1997; Boussetta et al., 2013), we assume that radiation transfer is the most relevant factor throughout the canopy. Thus, we use bulk values for other dynamic variables such as leaf temperature, vapor pressure deficit or $\mathrm{CO}_{2}$ mixing ratio, 
and keep them invariable throughout the canopy. Multilayer canopy models that have to resolve the surface energy balance and all dynamic variables at each canopy level are computationally more expensive. As a result, our multilayer approach is faster, while still considering radiation variations within the canopy.

\subsubsection{Conditional averaging}

The alteration in radiation by clouds affects the surface locally (Horn et al., 2015). It is therefore convenient to apply a two dimensional conditional averaging of surface properties dependent on cloud properties above. We classify the surface response according to cloud optical depth in bins of gradually increasing width: bins of 0.5 width between values of cloud optical depth $\tau=0$ and $\tau=5$, and in bins of 1.0 between values of $\tau=5$ and $\tau=10$ to obtain more information on the properties at the surface for cloud optical depths typical of shallow cumulus clouds (McFarlane and Grabowski, 2007; Slawinska et al., 2008). Three more bins are defined for thicker clouds: two bins with a width of 5 between $10<\tau \leq 15$ and $15<\tau \leq 20$ and a bin for $\tau>20$.

Since we are also interested in the properties of the clouds during the experiment, we make further use of a three-dimensional conditional average over the grid, similar to that used in other studies (Siebesma et al., 2003; Ouwersloot et al., 2013; Sikma and Ouwersloot, 2015). In this case, we mainly focus on the conditional averages for clouds $\left(q_{l}>0\right)$ and for the buoyant part of clouds or cloud cores $\left(q_{l}>0\right.$ and $\left.\theta_{v}>\overline{\theta_{v}}\right)$, where the overbar stands for domain average properties at each vertical level).

\subsubsection{Research strategy}

We reproduce a representative warm and initially clear early autumn day of September in The Netherlands developing a convective boundary layer. Surface and upper observations are initially obtained from observations at the Cabauw Experimental Site for Atmospheric Research (CESAR). The case has been adapted to allow the onset of active shallow cumulus (Vilà-Guerau de Arellano et al., 2014). First shallow cumulus develop between 10 and 11 UTC, after which a maximum cloud cover of $\sim 20 \%$ is reached between 13 and 15 UTC. The numerical experiments simulate 10 hours, from 7 UTC to 17 UTC (9 to 19 Local Time), with an initially well-mixed boundary layer of around 120 meters and with no prescribed horizontal wind. We define the boundary-layer height as the height at which the gradient in potential temperature $\theta$ equals $50 \%$ of the maximum vertical gradient (Ouwersloot et al., 2011). We make use of an all-or-nothing microphysics scheme, assuming condensation of all specific humidity above local saturation point and none if saturation is not reached. The surface consists of a homogeneous grassland of Leaf Area Index $\mathrm{LAI}=2$ with radiative and dynamic properties typical of short grass, and a vegeta- 
tion cover of $90 \%$. The domain is $24 \times 24 \times 5.4 \mathrm{~km}^{3}$ with a gridbox size of $50 \times 50 \times 12 \mathrm{~m}^{3}$. Such a vertical resolution ensures the explicit resolution of clouds and its dynamics.

We design three experiments: REF (reference or control), DIR (direct) and DIF (diffuse). The REF experiment calculates the amount of direct and diffuse radiation below the clouds using the Delta Eddington approximation (Sect. 3.2.3.1) and sets the response of the surface to radiation through the canopy scheme as described in Sect. 3.2.3.2 and with more detail in the supplemental material. The experiment DIR uses the same radiation below the cloud, but treats all radiation reaching the canopy as direct radiation. In turn, the DIF experiment considers all radiation reaching the canopy to be diffuse as long as there is a cloud above. With this three experiments we explore the sensitivity of the surface and boundary layer to the direct radiation and diffuse radiation ratio below clouds. Two additional experiments are carried out to put the aforementioned numerical experiments in perspective. The first one (AER) mimics the effect of downwards diffuse radiation due to Rayleigh scattering above the domain by converting $7 \%$ of the direct radiation, $S W_{d i r}^{T o D}$, to diffuse radiation, $S W_{d i f}^{T o D}$, at the top of the domain (Barbaro et al., 2014). This radiative effect at the surface is similar to that of a boundary layer with clouds and a significant presence of light-scattering aerosols (Yu et al., 2002; Barbaro et al., 2014). The second experiment, LAI5, is identical to the reference experiment but with a Leaf Area Index of 5. The goal of this experiment is to reveal the sensitivity of the surface-boundary layer system to a change in vegetation density, which impacts the penetration of direct and diffuse radiation in the canopy and, thus, the surface fluxes.

\subsection{Results and discussion}

\subsubsection{Impact of shallow cumulus at surface}

\subsubsection{Impact on surface fluxes}

Figure 3.1b shows the exponential decay in shortwave direct radiation, as stated in Eq. (3.8). There is a solar-angle dependent maximum in diffuse radiation for $\tau$ between $\tau=1$ and $\tau=8$ (Fig. $3.1 \mathrm{c}$ ). Increasing cloud thickness converts more direct to diffuse radiation, while at the same time reduces the overall radiation going through the cloud (Fig. $3.1 \mathrm{a}$ ). This increase in diffuse fraction is of critical importance for the feedbacks and interactions in our numerical experiments, since the cloud optical depth of the shallow cumulus created during the day ranges around those values. In fact, McFarlane and Grabowski (2007) observed that in the tropics the optical depth of the most common shallow cumulus ranges between $\tau=5$ and 10, a finding also supported through numerical experiments by Slawinska et al. (2008). To our knowledge,such a study on typical optical thickness of shallow cumulus has not been carried out for mid-latitudes, where our study focuses. The 


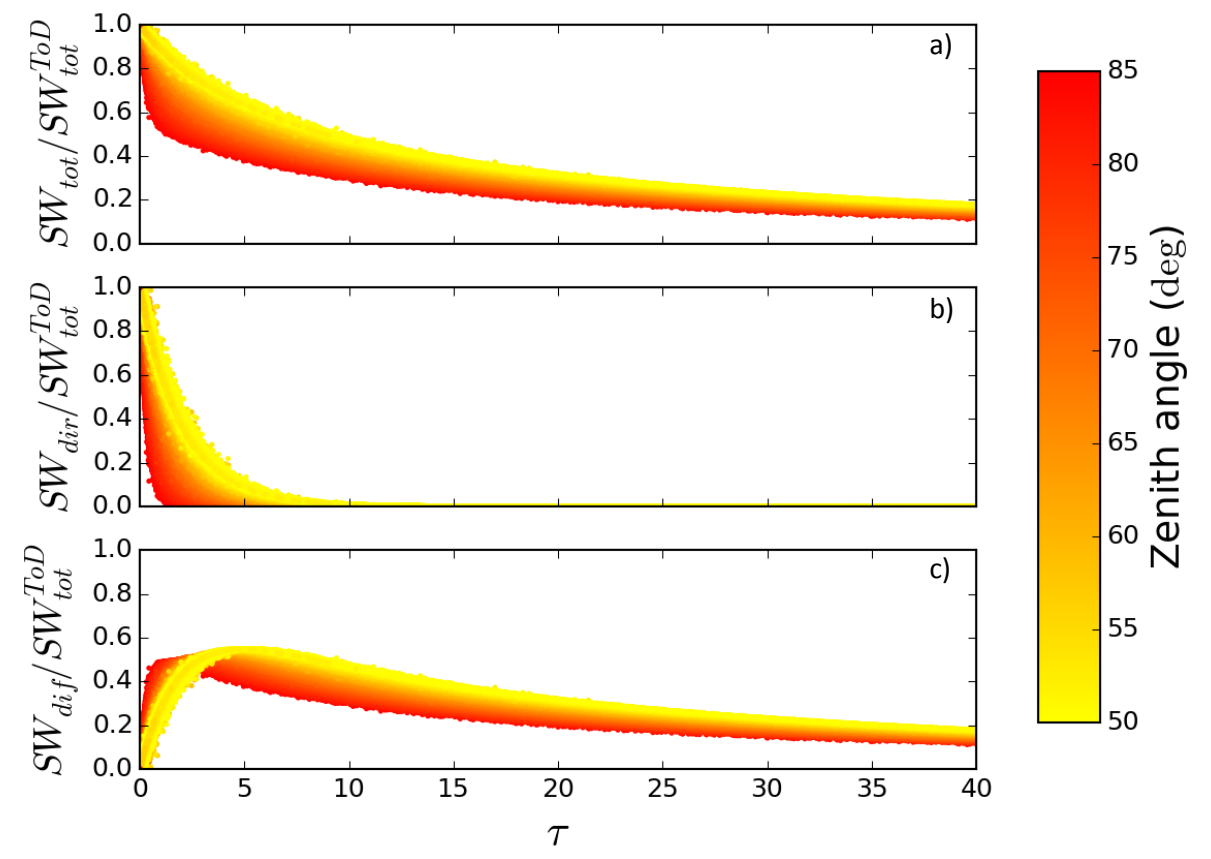

Figure 3.1: Ratio of total (a), direct (b) and diffuse (c) radiation at the surface to the total radiation at the top of the domain, $S W_{t o p}^{T o D}$, as a function of cloud optical depth, $\tau$, as obtained from the REF experiment. The gradual color shows the dependency on solar zenith angle, ranging from $\theta=50^{\circ}$ (yellow) at its zenith for the experiment day to $\theta=85^{\circ}$ (red).

results shown in Fig. 3.1 are consistent with the satellite measurements by Cheng et al. (2016), supporting the use of a broadband radiation scheme like the Delta-Eddington for our purposes.

The perturbation of radiation by clouds has an immediate effect at the surface turbulent fluxes. The presence of any cloud locally reduces the total radiation at surface: from around $15 \%$ for very thin clouds $(\tau<3)$, to almost $85 \%$ for very deep clouds $(\tau \sim 40)$ (Fig. 3.1 a). In our study we focus on the effect of shallow cumulus. Thus, we pay special attention to clouds with $\tau<8$. Fig. 3.2 depicts the evolution of a single cloud in the REF experiment, and shows how the surface responds according to the cloud thickness. Figures $3.2 \mathrm{a}$ and $\mathrm{b}$ illustrate the relation between the cloud optical depth and shortwave radiation, both direct and diffuse. For very thin clouds with $\tau<3$ (see the area at $x=400, y=750$ $\mathrm{m}$ in the first column in Fig. 3.2), the decrease in direct radiation $S W_{d i r}$ is to some extent compensated by the increase in diffuse radiation $S W_{d i f}$. In addition to the known higher penetrative capacity of diffuse radiation inside the canopy (Gu et al., 2003; Knohl and Baldocchi, 2008; Urban et al., 2012), more diffuse radiation is created inside the canopy by scattering of leaves (Goudriaan, 1977; Baldocchi et al., 1985). The more homogeneous spread of diffuse radiation in the vertical increases the stomatal conductance and decreases the vegetation canopy resistance for water vapor, $r_{v e g}$, as long as the total radiation at surface does not decrease dramatically (Fig. 3.2c). This decrease in vegetation resistance 

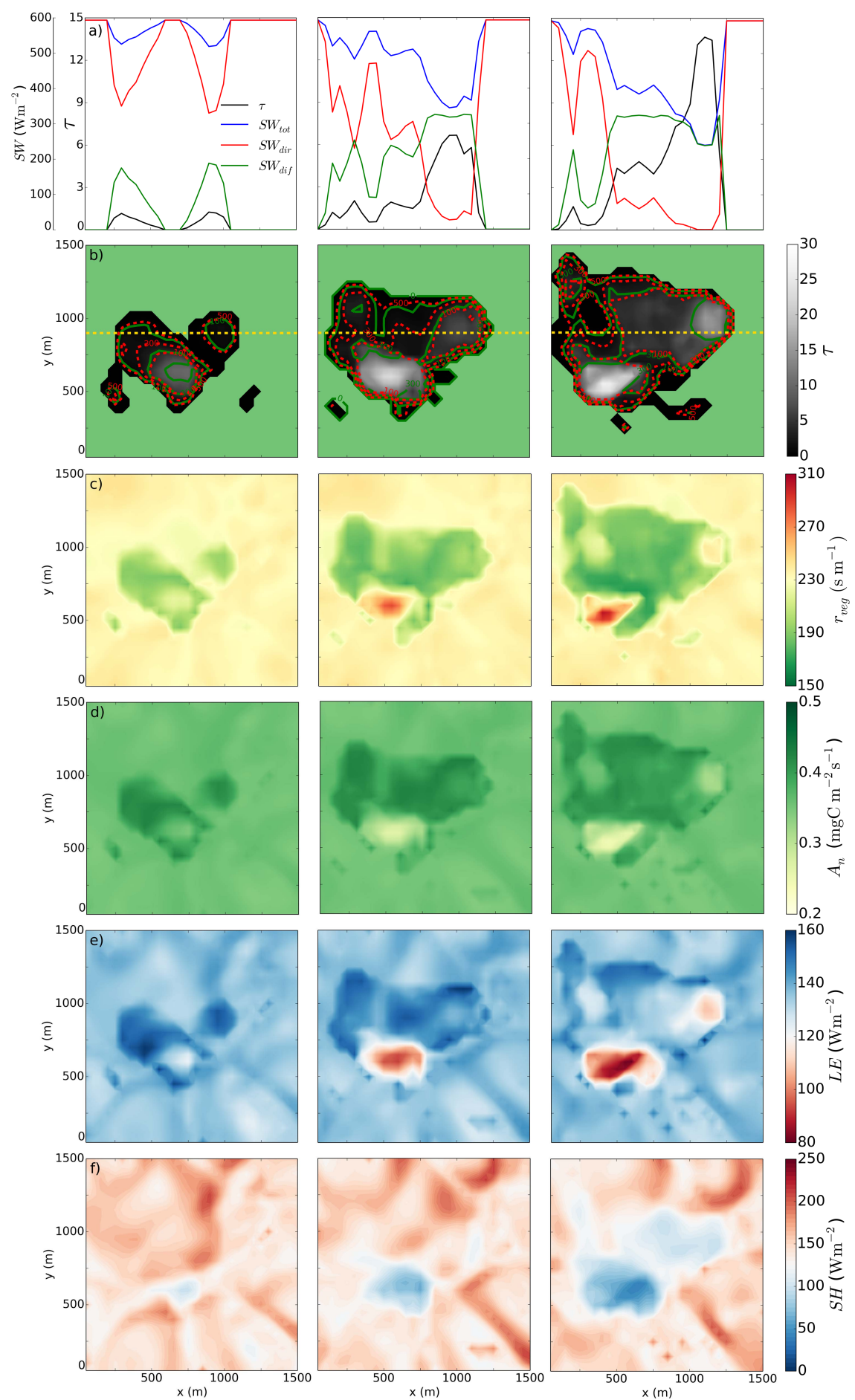

Figure 3.2: (a) Cloud optical depth, $\tau$ (black), shortwave direct, $S W_{d i r}$ (red), diffuse, $S W_{d i f}$ (green), and total radiation, $S W_{\text {tot }}$ (blue), along the cross-section (horizontal yellow dashed line) displayed in b. (b) Instantaneous horizontal cross-sections of cloud optical depth $\tau$ (shades) with shortwave direct and diffuse radiation $S W_{d i r}$ and $S W_{d i f}$ in dashed red and full green lines, respectively. Clear sky has been plotted in green. (c) Vegetation canopy resistance, $r_{v e g}$. (d) Net carbon assimilation, $A_{n}$. (e) Latent heat flux, LE. (f) Sensible heat flux, SH. These snapshots are instantaneous cross-sections of a cloud generated in experiment REF. The first column depicts the situation at 11:57 UTC, with next columns advancing 2 minutes compared to the previous one. 
due to diffuse radiation is a scale-dependent property. While it is not found at leaf level (Brodersen et al., 2008), this increase is present when we upscale from the leaf to canopy level.

Although the total radiation at the surface is locally reduced compared to cloudless conditions, the higher efficiency of diffuse radiation in the canopy explains the growth in $A_{n}$, visible as darker green patches in Fig. 3.2d. We make a more detailed analysis on the factors driving $A_{n}$ variations in Fig. 3.6. Linked to $A_{n}$, latent heat flux increases under thin clouds: while plants open their stomata to take up $\mathrm{CO}_{2}$ releasing water vapor becomes unavoidable. Thus, water and carbon fluxes are related through the stomatal opening.

In the conditions in Fig. 3.2, $r_{\text {veg }}$ is on average larger than $r_{a}$. However, spatial variations on $r_{v e g}$ are as large as $150 \mathrm{~s} \mathrm{~m}^{-1}$ within the surface shaded by the sampled cloud, while $r_{a}$ has a more limited variability of around $40 \mathrm{~s} \mathrm{~m}^{-1}$, and not corresponding exactly to the cloud shading, since surface horizontal wind and updraft locations largely determine $r_{a}$ (Sikma et al., 2018). Thus, the decrease in $r_{v e g}$ due to diffuse radiation drives $L E$ variations. However, $L E$ shows weaker enhancement under the thin parts of the cloud than $A_{n}$. This is due to the fact that the vapor pressure deficit (VPD) is also reduced in the shaded colder surface, thus decreasing the last factor in Eq. (3.1). Although the stomata opening is also indirectly dependent on VPD, its sensitivity is much weaker, thus not being of much importance for $r_{v e g}$ or $r_{v e g_{\mathrm{CO}_{2}}}$. Sensible heat flux, depicted in Fig. 3.2f, shows a decrease for all cloud thicknesses. This is due to the fact that sensible heat flux is driven by the surface temperature (Eq. (3.2)), which is governed by total radiation and, thus, decreases for any cloud. The fact that $S H$ depends on $r_{a}$, and not on $r_{v e g}$, explains the less clear patterns in Fig. $3.2 \mathrm{f}$ compared to, e.g. $L E$, for which the effect of cloud shading is clearly discernible. However $A_{n}$ and $L E$ actually decrease when the cloud optical depth grows further (see the area at $x=400, y=500$ at the third column in Fig. 3.2). Under such conditions, direct radiations almost vanishes and the diffuse radiation is insufficient to maintain the clear-sky high $A_{n}$ and $L E$.

To quantify the changes at the surface along the day, we show the temporal variation in vegetation carbon uptake and surface fluxes under clear skies and for a few cloud optical depth bins in Fig. 3.3. For thick clouds $(\tau>20)$, both latent and sensible heat fluxes are reduced on average compared to clear sky conditions (Fig. 3.3b, c), as also found by Lohou and Patton (2014) and Horn et al. (2015). A similar effect is found in net carbon assimilation by vegetation. Relevant for our research is that instantaneously responding vegetation under very thin clouds $(\tau<3)$ has a stronger carbon uptake, by as much as $9 \%$, compared to that of clear sky conditions until 16:30 UTC (Fig. 3.3a). The photosynthesis rate is enhanced due to the large amount of diffuse radiation (Fig. 3.1c). Even though total radiation is reduced under any cloud, the higher absorption efficiency of diffuse radiation by canopies overcomes the total reduction in light intensity 


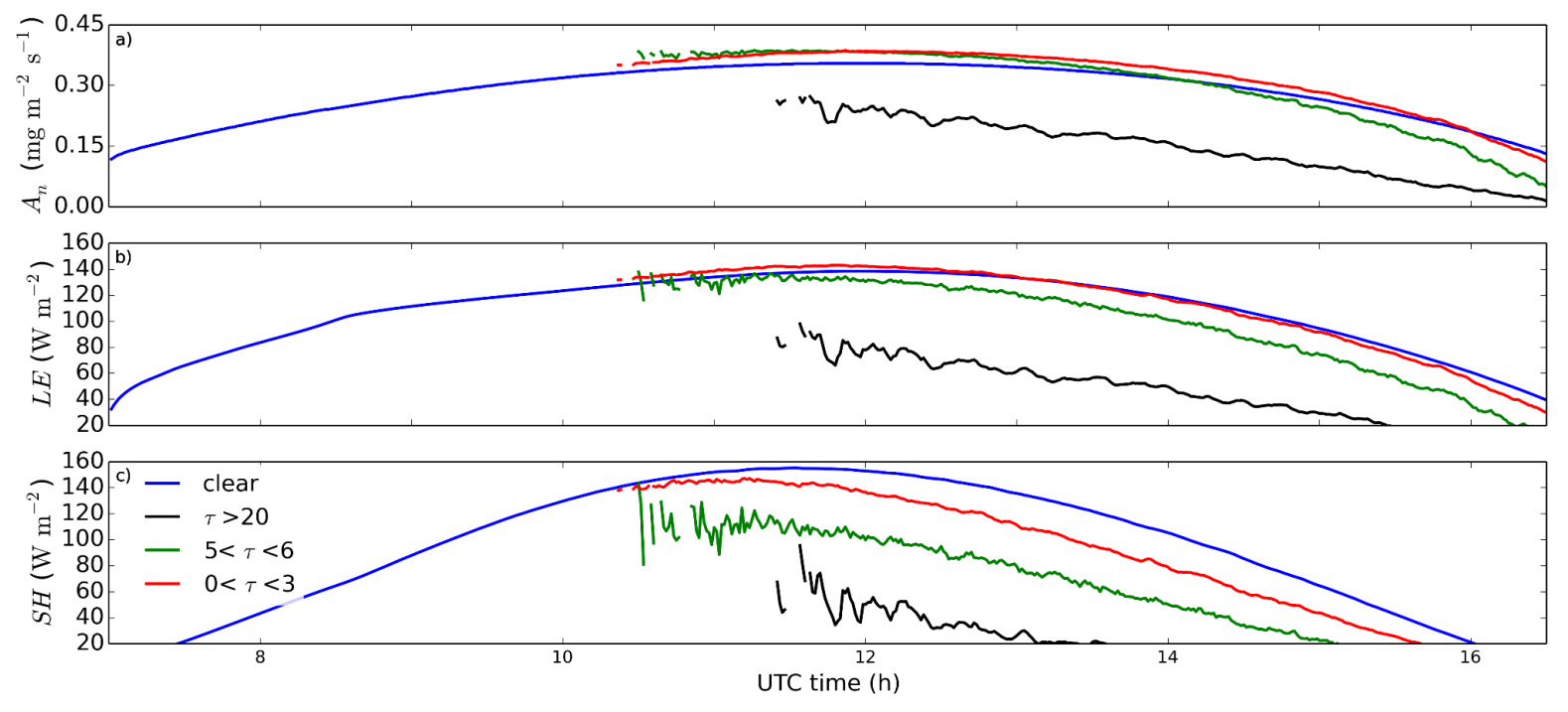

Figure 3.3: Time series of net carbon assimilation, $A_{n}$ (a), latent heat flux, $L E$, (b) and sensible heat flux, $S H$, (c) classified according to 4 conditional averages: clear sky (blue), and three cloud optical depth ranges: $\tau<3.0$ (red), $5<\tau<6$ (green) and $20<\tau$ (black). The onset of shallow cumulus clouds occurs at 10:37 UTC.

and decreases the average vegetation resistance by as much as $11 \%$ (not shown) at around 12 UTC. Although $r_{a}$ increases (by about $4 \%$ at 12 UTC, not shown), the larger reduction in vegetation resistance drives the increased activity of vegetation under very thin clouds. Consequently, we also find a latent heat flux that is marginally larger than under clear sky, provided $\tau<3$, from 10:30 to 13:30 UTC. This difference between clear sky and very thin clouds is smaller than for $A_{n}$ and reaches maximum values of only $4 \%$ (see interval between 10:30 and 12:00 UTC at Fig. 3.3b) due to the fact that the vapor pressure deficit (VPD) is also reduced in the shaded colder surface. Since the sensible heat flux depends only on factors independent of radiation partitioning (air-surface temperature gradient and $r_{a}$ ), it decreases under thin clouds too, as observed in Fig. 3.2. In other words, the reduction in surface energy by thin clouds is not found in $L E$, so the sensible heat flux must decrease. Values for intermediate cloud optical depth, with $\tau$ between 5 and 6 , show similar latent heat flux compared to that under clear skies between 10:30 and 11:30 UTC, and lower afterwards.

To further quantify the dependencies of surface fluxes and net carbon uptake on cloud thickness, Fig. 3.4 shows one-hour averaged carbon uptake, latent heat and sensible heat fluxes as a function of cloud optical depth between 11 and 12 UTC for the experiments REF and DIR and normalized by clear sky values. We first define a few relevant concepts. We call enhanced regime to the range of cloud optical depths at which we observe enhanced surface exchange. Threshold $\tau$ is defined for each flux as that cloud optical depth for which the flux $\left(L E\right.$ or $\left.A_{n}\right)$ equals its clear sky value. The maximum $\tau$ is the cloud optical depth at which we observe maximum values of surface exchange. 

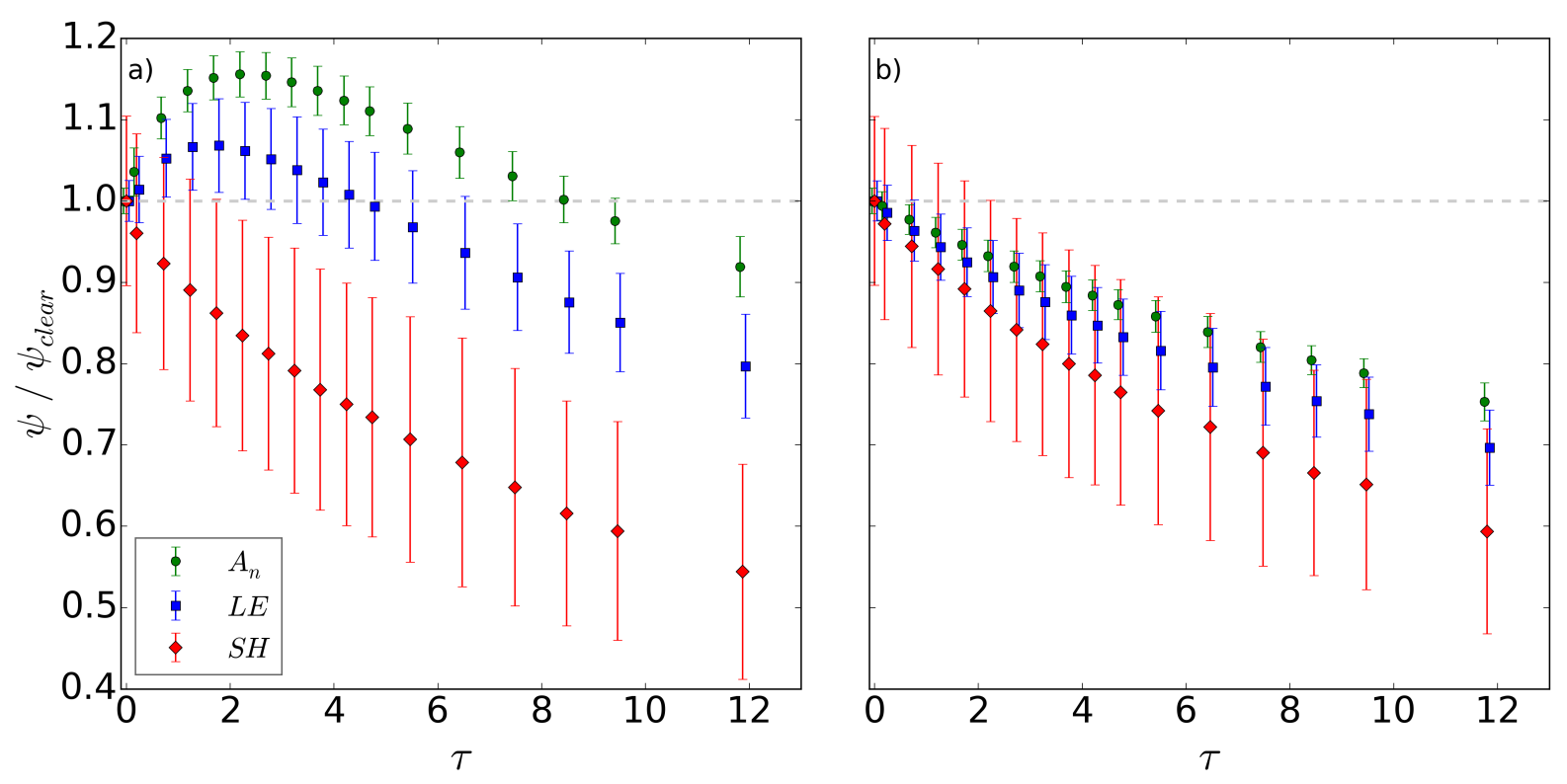

Figure 3.4: (a): mean net carbon assimilation, $A_{n}$ (green), latent heat flux, $L E$ (blue), and sensible heat flux, $S H$ (red), averaged and binned according to cloud optical depth, $\tau$, above between 11 and 12 UTC and normalized over clear sky values for the REF experiment. (b) As in a, but for the DIR experiment. The vertical bar lengths give two times the standard deviation from the mean.

The enhanced regimes show that the character of diffuse radiation is able to compensate in $A_{n}$ and $L E$ for the overall reduction in radiation at the surface for thin clouds. Figure $3.4 \mathrm{~b}$ shows that this enhancing effect is not present when all radiation is direct. This confirms the finding that thin clouds enhance carbon uptake and evapotranspiration by providing a favorable combination of direct and diffuse radiation. Vegetation net carbon assimilation at REF experiment shows larger values (as much as 18\%) than under clear sky for cloud optical depth below 9 . At $\tau \simeq 9$ we find the threshold $\tau$ for $A_{n}$. For latent heat flux the enhanced regime is smaller, and its threshold $\tau$ is around between 4 and 5 . The maximum latent heat flux is about $9 \%$ larger than under clear sky. Consistent with what was shown in Fig. 3.3, the sensible heat flux decreases monotonically with increasing cloud thickness for both cases: as less total radiation reaches the surface, surface temperature decreases and accordingly does the sensible heat flux. Interestingly, the sensible heat flux shows systematic slightly lower values (less than 5\%) for the REF case compared to DIR under any cloud due to the increase in $L E$. This small increase in $S H$ for the DIR case is interpreted as follows: the additional direct radiation present in DIR instead of the diffuse radiation in REF is not absorbed by the canopy since it does not penetrate that deep in the canopy. Instead, it is directed to light-saturated leaves, decreasing the latent heat flux and increasing the surface temperature and, thus, the sensible heat flux (see Eq. (3.2)) compared to the REF experiment. 

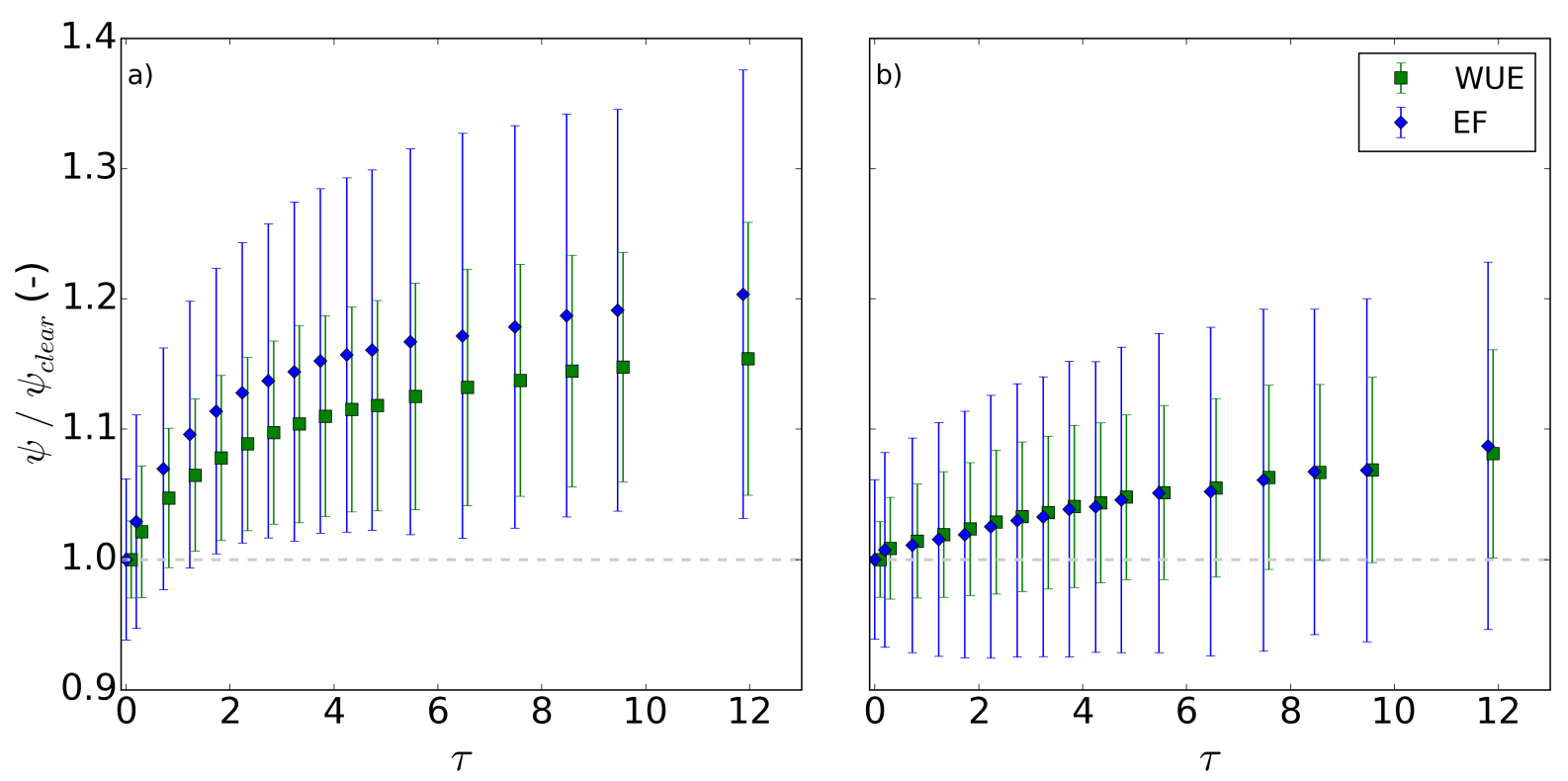

Figure 3.5: As in Fig. 3.4 but for evaporative fraction, $E F$ (blue), and water use efficiency, $W U E$ (green), for (a) REF and (b) DIR experiments.

In Fig. 3.4a an interesting effect is observed: at this time of the day, thin and thick clouds enhance and decrease plant activity, respectively, thus acting in opposing directions regarding surface responses. This means that the presence of thin and thick clouds may lead to similar domain-averaged carbon uptake or surface fluxes at situations where the spatial variability within the domain clearly differs. Note that the thresholds and maximum $\tau$ values vary during the day since the solar angle and surface conditions are not constant during the daytime, thus affecting this compensation effect.

For a quantification on the relative changes in Fig. 3.4 we show the evaporative fraction, $E F=\frac{L E}{L E+S H}$, and the water use efficiency, $W U E=\frac{A_{n}}{L E}$, of experiments REF and DIR in Fig. 3.5. Regardless of whether radiation has both diffuse and direct components or only direct, both $W U E$ and $E F$ increase under the presence of clouds. A (linear) increase of $E F$ is explained by the more pronounced decline of $S H$ than that of $L E$ when cloud thickness increases regardless of the character of light (Fig. 3.4a,b). The contribution of diffuse radiation shifts this shape to a more logarithmic-like curve, especially increasing $E F$ for the enhanced regime (Fig. 3.5a). Since the threshold $\tau$ for $L E$ lies around $\tau=5$, the curve above that cloud thickness in Fig. 3.5a shows a relative increase similar to that of the DIR experiment. Overall, EF is at least $10 \%$ larger for the REF compared to the DIR case under any cloud thickness. This suggests that, considering the entire domain, the $E F$ is positively affected not only by the reduction of total radiation by clouds, as found by Lohou and Patton (2014), but also by the presence of diffuse radiation. The $W U E$ behaves similarly: even for the DIR experiment there is a (linear) growth for increasing $\tau$ due to reduction of radiation at surface (Fig. 3.5b), but the presence of diffuse radiation 
amplifies the rise in $W U E$ for the enhanced regime $0<\tau<8$.

\subsubsection{Impact on carbon assimilation budget}

In order to disentangle how components in the plant physiological model contribute to the overall increase of carbon assimilation by plants, we perform an off-line study using the mixed-layer model CLASS. The conditions for the experiment are set as similar as possible to those of our LES study. In addition, we prescribe the onset, thickening and disappearance of a very thin cloud $(\tau=1)$.

The plant physiological model in CLASS is identical to the one used in DALES and described in the supplemental material, and calculates the net carbon uptake at canopy level according to Eq. (3.3). A budget analysis enables us to understand the contribution of each component of $A_{n}$ to the cloud-driven change in radiation at the surface around noon for a cloud thickness within the optimum region shown in Fig. 3.4. It must be noted that we do not intend to characterize the causes and consequences that drive the $A_{n}$ changes, as the feedbacks and interrelations between plant parameters and variables make such a description quite complex. Instead, with this analysis we aim to depict qualitatively the reaction of the main components in the plant-atmosphere system to fast changes in radiation over time.

The numerator in Eq. (3.3) represents the $\mathrm{CO}_{2}$ gradient between the plant and its surrounding environment. The aerodynamic resistance governs the efficiency of transport between leaf and the surrounding atmosphere. This depends on horizontal wind speed (and convective velocity $w_{*}$ in case of no wind), and is modulated for different stability regimes. $r_{v e g_{\mathrm{CO}_{2}}}$ gives the capacity of plant stomata (upscaled at canopy level) to exchange $\mathrm{CO}_{2}$ with the environment. Since we are interested in the contribution of each factor to the variation in $A_{n}$, we differentiate Eq. (3.3) to obtain a budget with three contributions:

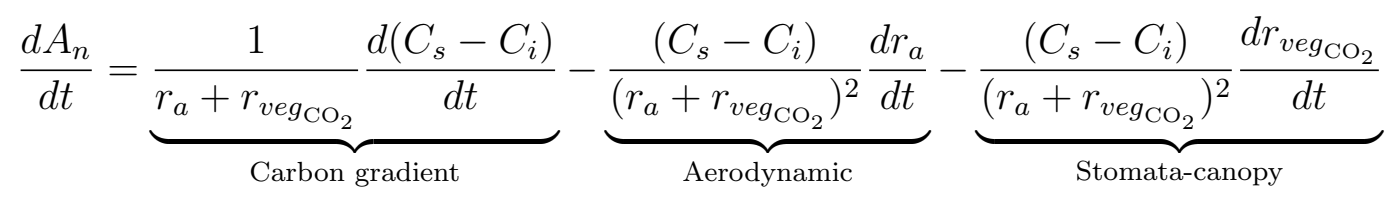

We name the terms following the derivative in time: plant-atmosphere carbon gradient, aerodynamic and stomata or canopy resistance terms. The first term on the right hand side of Eq. (3.9) represents the change in carbon assimilation due to a variation in the $\mathrm{CO}_{2}$ gradient between the atmosphere and inside the leaf. An increase (decrease) over time in such gradient implies an increase (decrease) in carbon assimilation. The second term on the right hand side accounts for the effect of atmospheric dynamics on the carbon assimilation. Being so, an increase (decrease) in aerodynamic resistance over 

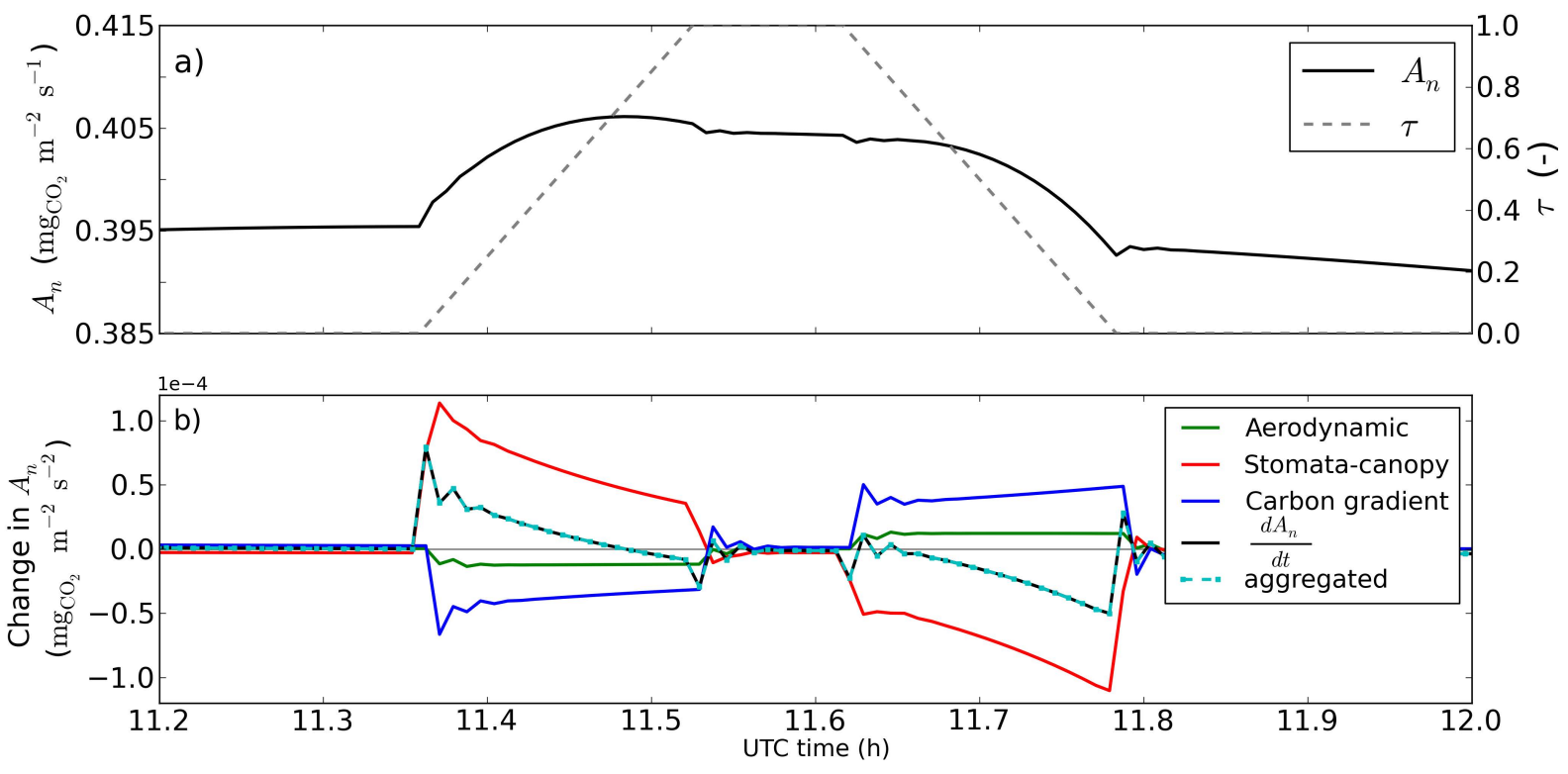

Figure 3.6: (a) Net carbon assimilation calculated with CLASS (full line) and prescribed cloud optical depth (dashed line). (b) Time derivative of $A_{n}$ (black line), aggregate (dashed blue line) and each of the components in the RHS of Eq. (3.9): carbon gradient (dark blue), aerodynamic (green), and stomata-canopy (red) components as obtained from the CLASS model. The conditions are constrained by the LES results and correspond to average values at 12:00 in experiment REF.

time, possibly related to a decrease in wind speed or higher atmospheric stability, would decrease (increase) the carbon uptake. The third term on the right hand side shows the relation between the vegetation resistance to $\mathrm{CO}_{2}$ transport and $A_{n}$. A positive (negative) change in $r_{v e g_{\mathrm{CO}_{2}}}$ due to a closing (opening) of stomata in the canopy would decrease (increase) the carbon assimilated by plants.

Figure 3.6a shows an enhancement in $A_{n}$ as the cloud optical depth increases, as expected from the optimum range in Fig. 3.4. The stomatal component is the only term contributing to this enhancement of carbon assimilation when the cloud appears. Since the increase in diffuse radiation compensates for the reduction in total radiation, the vegetation resistance is decreased, lowering the resistance to $\mathrm{CO}_{2}$ exchange. The colder surface by reduced total radiation decreases the VPD. This decrease correlates with increasing $C_{i}$ (Jacobs and de Bruin, 1997), thus weakening the $\mathrm{CO}_{2}$ gradient. Thus, the temperature-driven reduction on the $\mathrm{CO}_{2}$ gradient decreases carbon assimilation or, after adding all components in Eq. (3.9), decreases the carbon assimilation enhancement driven by the stomata component. Similarly, although at smaller scale, the dynamic component hampers the enhanced carbon assimilation. This is due to a decreased total radiation at surface, which cools the surface and consequently leads to weaker buoyancy flux and related convective motions and, thus, increases aerodynamic resistance, $r_{a}$. The symmetric 
decrease in cloud optical depth after 11.6 UTC shows an analogous impact to that explained for the increasing optical depth, with each factor contributing with opposite sign. For thicker clouds, the temperature-driven negative effects $\left(\mathrm{CO}_{2}\right.$ gradient and dynamic components in Eq. (3.9)) strengthen, while the positive impact of the stomatal component is reduced due to lower total radiation, and it changes sign under thicker clouds. Thus, under thick enough clouds we find a reduction in carbon assimilation.

In the additional DALES experiments, an increase in LAI (from 2 to 5) increases the absorbed radiation by the canopy. The increase is mostly due to the larger number of leaves keeping most of the light inside the canopy. The amount of direct light converted to diffuse light inside the canopy increases with increasing LAI (from $25 \%$ to $40 \%$ of available direct radiation for $L A I=5$ ), but the direct light converted to diffuse light is not the driving factor for the increased absorbed radiation. In particular, the diffuse radiation causes the latent heat and $A_{n}$ to rise, while it lowers the sensible heat flux compared to the REF experiment (Fig. 3.3). However, the differences between clear skies and the cloud thickness bins (Fig. 3.3 for REF experiment) remain fairly similar. The dependence of surface response to cloud thickness between 11:00 and 12:00 UTC is very similar to that of Figs. 3.4a and 3.5a, with slightly lower $E F$ and larger $W U E$, but still above clear sky values.

When accounting for the fraction of diffuse radiation (7\% of the total radiation) at the top of the boundary layer due to Rayleigh scattering above (AER experiment), we still find an enhanced regime with higher $L E$ and $A_{n}$ under the (thin) clouds than under cloudless sky. Because there is already some diffuse radiation in clear sky conditions, the threshold and maximum $\tau$ are, however, lower than in Fig. 3.4a. Thus, the relative differences between clouds and clear sky are less pronounced (about 1\% lower increase in $E F$ and very similar $W U E$, not shown).

\subsubsection{Impact of surface on boundary layer and shallow cumulus}

In Sect. 3.3.1.1 it was already shown that the partition between direct and diffuse radiation plays a critical role on the surface response. Therefore, it is interesting to investigate whether these changes at the surface feedback to the boundary-layer dynamics and subsequently to cloud characteristics, such as their formation and vertical development. Focusing on the whole domain, Horn et al. (2015) found significant changes in domainaveraged sensible and latent heat flux when the cloud shading was considered. Yet they did not quantify the separate contributions of direct and diffuse radiation. Although small spatial variations in surface fluxes are observed starting at the time first clouds develop (11 UTC), we find that the domain-averaged $S H$ and $L E$ do not change significantly between experiments, as we account for the cloud shading in all our experiments. Domain averaged vegetation carbon uptake shows larger differences, although still below $5 \%$ 


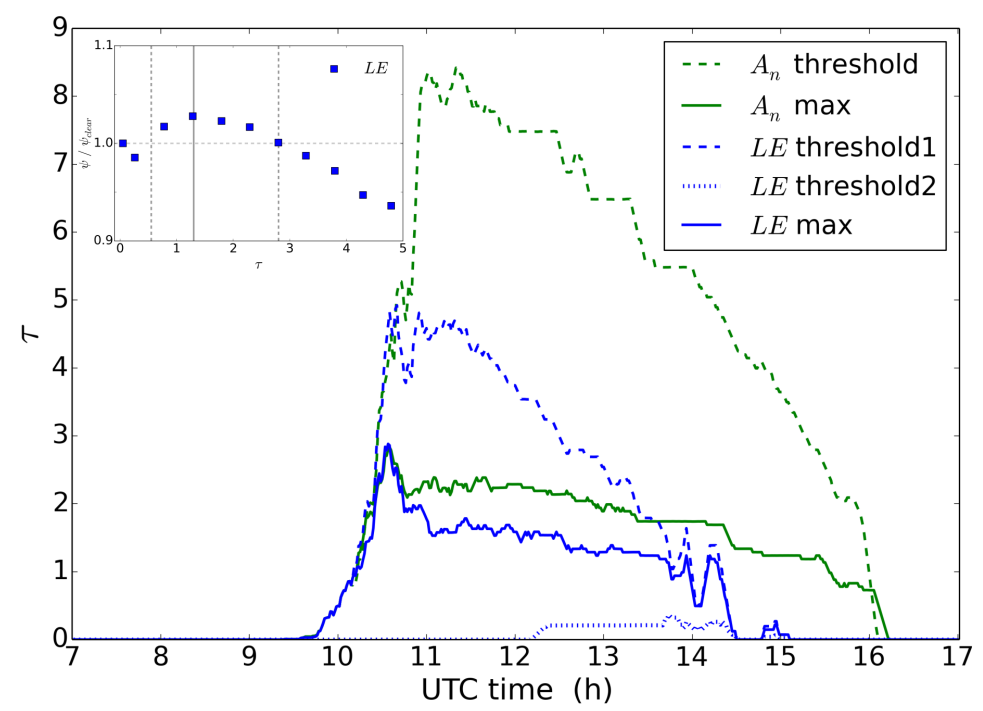

Figure 3.7: On the top-left corner subfigure: same as in Fig. 3.4a but instantaneous, at 13 UTC and only for latent heat flux, $L E$. Vertical dashed grey lines give the two thresholds present at that time, and vertical grey full line the $\tau$ at which maximum $L E$ is found. On the main figure: cloud optical depth at which values for latent heat flux, $L E$, (blue) and vegetation carbon uptake, $A_{n}$, (green) are highest (full line) and equal to those under clear sky (dashed) in the REF experiment. The dotted line shows the additional threshold $\tau$ that appears for $L E$ under very thin clouds in the afternoon (see subfigure for $0<\tau<1$ ).

compared to the REF experiment (not shown).

The reason for smaller differences in evapotranspiration than in $A_{n}$ is the same as observed in Fig. 3.3: thin clouds decrease vegetation resistance while cooling the surface. This cooling reduces the VPD, counterbalancing the increase in $L E$ by stomatal opening. Thus, the enhancement compared to clear sky latent heat flux shown in Fig. 3.4 is, at its maximum, of around $10 \%$. This is in contrast to the maximum of $20 \%$ found for the vegetation carbon assimilation. Due to the same reason, the enhanced regime is lower for latent heat than for carbon uptake, as already seen for the 11-12 UTC average in Fig. 3.4 .

The main reason for the little difference in domain averaged surface fluxes lies on the limited enhancement of atmosphere-vegetation exchange (less than $10 \%$ and $20 \%$ at their maxima for $L E$ and $A_{n}$, respectively) in the REF experiment. In addition, the threshold $\tau$ 's are dynamic and vary during the day according to solar angle, amount of radiation and surface conditions such as temperature, $\mathrm{CO}_{2}$ concentrations and vapor pressure deficit. Adding the temporal evolution of these thresholds, shown in Fig. 3.7, to the limited enhancement of $A_{n}$ and $L E$ limits the duration of the enhanced effect for latent heat and, to a lesser degree, for carbon uptake. Whereas the enhanced regime for $A_{n}$ is present before 10 UTC (when first clouds arise) and until after 16 UTC, the regime for latent heat 


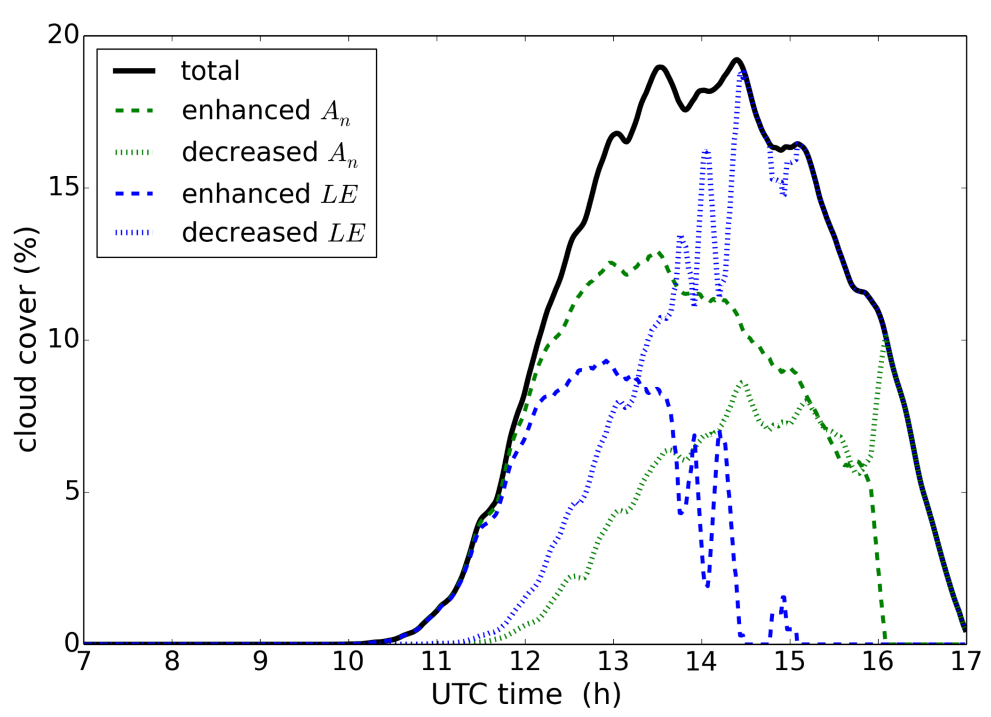

Figure 3.8: Total cloud cover (black), and clouded area depending on whether carbon assimilation, $A_{n}$, (green) and latent heat flux, $L E$, (blue) are enhanced (dashed) or decreased (dotted) compared to clear sky values in the REF experiment. Note that the addition of enhanced and decreased areas equals the total cloud cover except for rounding errors.

only lasts between 10 UTC and 14:30 UTC. Moreover, in the afternoon the latent heat under clouds characterized by values $\tau<1$ is lower compared to clear sky, thus introducing also a lower threshold $\tau$ and narrowing the enhancement regime, (see subfigure in Fig. 3.7). In addition to the limited effect on time and scale, the enhanced latent heat flux and carbon assimilation are also limited in space. The low thresholds $\tau$ and maximum values for latent heat flux shown in Fig. 3.7 imply that less of the domain is below clouds with an optical thickness within the enhanced regime. The cloud cover corresponding to the enhanced $L E$ regime is much lower than for the carbon uptake, $A_{n}$, after 11:30 UTC (Fig. 3.8). In fact, after 14:30 UTC there is barely any cloud under which enhanced latent heat is found. As for $A_{n}$, we find, at its maximum, only $13 \%$ of the whole domain to show enhanced $A_{n}$ (Fig. 3.8). The compensating effect between thin and thick clouds also limits strong impacts on the atmosphere, as we observe large sub-areas of the clouded domain, especially in the afternoon and for $L E$, to decrease the atmosphere-vegetation exchange (Fig. 3.8). Thus, the effect of enhanced evapotranspiration and carbon uptake due to an optimal combination of diffuse and direct radiation is limited on scale (by limited maximum values), time (by a limited lifetime of the enhanced regime) and on space (by a low corresponding cloud cover).

Given the very similar response of the surface as a whole to cloud-driven changes in radiation, the boundary-layer height evolves almost identically for the three experiments. The boundary-layer top starts rising from an initial height of 120 meters at sunrise, around 8:15 UTC. The appearance of clouds, at around 10 UTC in our experiments, decelerates the mixed-layer growth by venting air containing high moisture and momentum within 
the mixed layer to higher levels (Neggers et al., 2006; van Stratum et al., 2014). The maximum boundary-layer height, similar for three experiments, reaches around 1950 meters at 16:30 UTC (not shown). In addition, vertical profiles of potential temperature and specific humidity at the subcloud layer are almost identical between the three experiments. Domain averaged heat and humidity fluxes along the boundary layer show small differences between experiments and are similar to previous studies (Brown et al., 2002; Siebesma et al., 2003). These findings support the results by Patton et al. (2005), who found little impact of short-scale, i.e. $2 \mathrm{~km}$, surface heterogeneities on vertical flux profiles. In all experiments, first very thin convective clouds appear at around 10 UTC. As the day evolves, cloud cover increases with shallow clouds growing larger both in horizontal and vertical scales (not shown). In the afternoon, the number of clouds decreases, although the large extension of the remaining ones keeps the cloud cover fairly similar. The cloud cover oscillates around a maximum value of almost $20 \%$ in the afternoon for the three experiments (Fig. 3.8 for REF), and we find a similar maximum cloud area fraction (the maximum cloud cover in a single vertical level) and maximum cloud core area fraction for the different experiments, thus suggesting that the strength of updrafts and mass flux within the clouds is not affected by changes at the surface.

The three-dimensional conditional averaging method for clouds presented in Sect. 3.2.4 allows us to carry out a much more detailed and precise analysis on the characteristics of clouds. In Fig. 3.9a we show the water content of cloud and cloud cores between 14 and 14:20 UTC in the three experiments REF, DIR, DIF, when the cloud cover is maximum. All experiments show a larger content on cloud cores than in clouds, as already documented in literature (Siebesma et al., 2003; Zhao and Austin, 2005). The direct or diffuse character of light does not affect the properties of clouds, as shown in Fig. 3.9a. The differences between experiments in $q_{l}$ are in the order of the standard deviations within each experiment: below $4000 \mathrm{~m}$ differences between experiments reach maximum values of $0.15 \mathrm{~g} \mathrm{~kg}^{-1}$, while the standard deviation within each experiment is almost double the relative differences between experiments. In Fig. 3.9b we observe that the mass flux vertical profile by cloud cores, i.e. the additional vertical velocity induced by the presence of clouds (Heus and Jonker, 2008; Ouwersloot et al., 2013), is similar between these experiments. It shows a maximum at around 2000 meters, which coincides with the maximum cloud core area at the same time (not shown). The small difference in the DIF experiment is due to the limitation of the domain extension, and can disappear or even change sign depending on the averaging time. All three experiments show a similar evolution of the maximum mass flux during the day (not shown).

The differences in treatment of radiation between the experiments allow us to give an indication for future parameterizations: while the partitioning of diffuse and direct components has some impact at the surface, locally affecting the surface fluxes and vegetation performance, it does not noticeably impact the dynamics of the boundary layer and the formation of low convective clouds. 

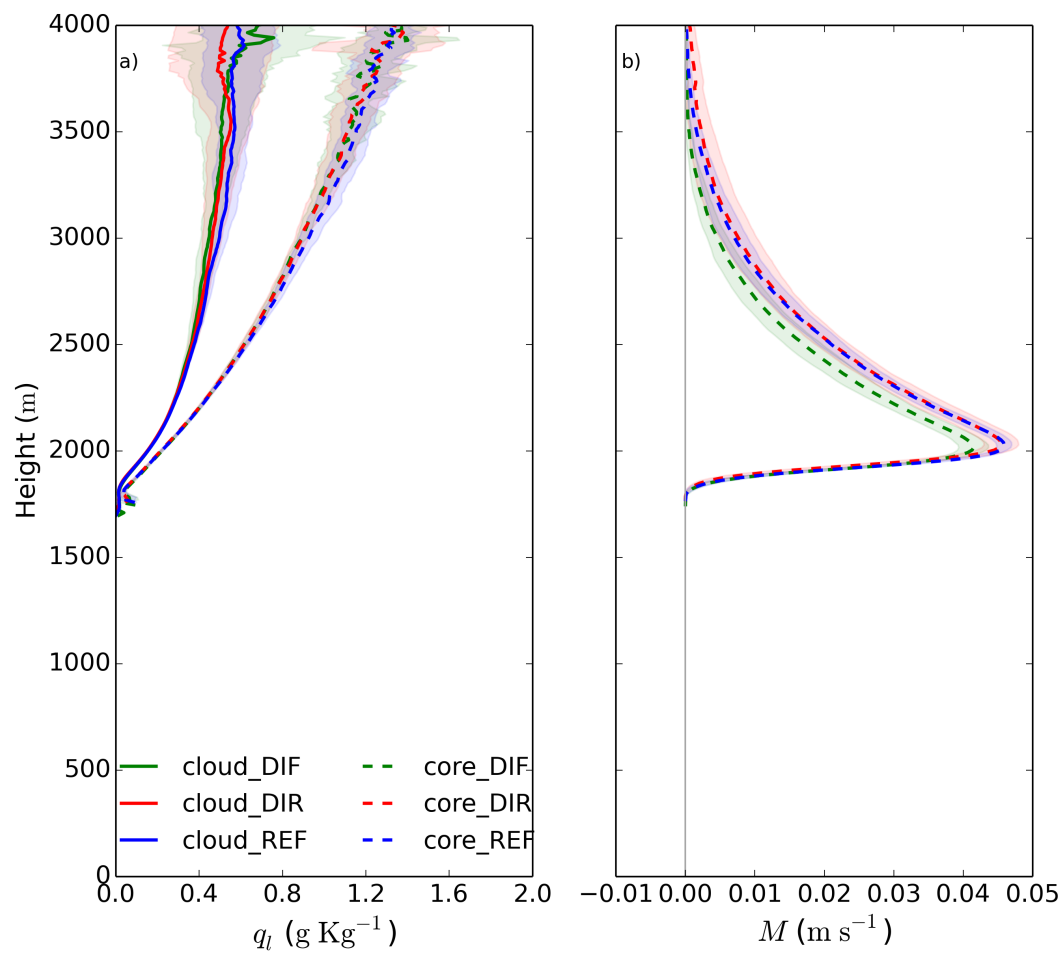

Figure 3.9: Conditional average on clouds (full) and cloud cores (dashed) of liquid water mixing ratio (left) and mass flux (right) for the REF (blue), DIR (red) and DIF (green) experiments. The profiles have been averaged between 14:00 and 14:20 UTC. Standard deviation for the temporal average is given in shades.

The additional LAI5 experiment yields a lower boundary layer, cloud height and slightly larger water content on clouds (not shown) than for lower LAI (REF experiment). These differences are driven by the larger transpiration due to a denser canopy. Although domain-averaged surface fluxes are very similar between AER and REF, all clouds after 13:00 UTC reduce the latent heat flux compared to clear sky in AER. This is due to the very narrow enhanced regime for $L E$ in AER. We find a slightly lower ABL height and very similar cloud cover and cloud characteristics for this additional experiment.

\subsection{Summarized conclusions and recommenda- tions}

By coupling radiation, surface and turbulence processes in the Dutch Atmospheric Large Eddy Simulation (DALES), we study the effects of direct and diffuse radiation in a diurnal boundary layer over vegetation. We pay special attention to how the direct and 
diffuse partitioning perturbed by clouds is further disturbed in the canopy modifying photosynthesis, plant evapotranspiration and sensible heat flux. We design three idealized experiments to clarify the role of diffuse and direct radiation at the vegetated canopy, boundary layer and clouds under free convective conditions (no mean horizontal wind). To disentangle the effects of cloud thickness on direct and diffuse radiation, we apply a conditional averaging at the surface dependent on the cloud optical depth above.

By calculating the radiative transfer in clouds with the delta-Eddington radiative scheme we find a decrease in total radiation at the surface under all cloud thickness. The fraction of diffuse radiation, however, increases for thin clouds and peaks at values of cloud optical depth between 1 and 8, typical values for shallow cumulus clouds. The sensitivity of radiation to cloud optical depth leads to different partitions of direct and diffuse radiation at the canopy top, which has an impact on the transfer of radiation inside the canopy, as diffuse radiation has larger penetrative capacity. In consequence, vegetation resistance is reduced under very thin clouds (as much as $11 \%$ at 12UTC), thus allowing for a larger exchange of water vapor (latent heat flux) and $\mathrm{CO}_{2}$ (vegetation carbon assimilation). We find a compensating effect between thin and thick clouds over the domain: thin clouds enhance the latent heat flux and photosynthesis, whereas thick clouds decrease them. Diffuse radiation from clouds is also responsible for the photosynthesis-driven increase in water use efficiency and evaporative fraction compared to the case when all radiation is direct.

To support the DALES results, we perform an off-line analysis using an atmospheric mixed-layer model to isolate and break up the complexity on the response of vegetation to prescribed cloud shading. We find changes in stomatal opening to lead the increased carbon uptake under very thin clouds. The $\mathrm{CO}_{2}$ gradient between the leaf and its environment, and the aerodynamic resistance are of secondary relevance. Yet they effectively reduce the enhancement of carbon uptake under very thin clouds.

As for the impact of dynamic surface heterogeneities on the boundary-layer structures, diffuse radiation does not play a relevant role in our experiments: similar domain averaged latent and sensible heat fluxes, boundary-layer heights and cloud characteristics are found regardless of the character of radiation under clouds. Slightly larger differences are found for domain-averaged carbon uptake, although below $5 \%$. The reduced difference on domain-averaged responses to diffuse radiation has several reasons. At its maximum, the latent heat flux and carbon assimilation enhancement is $10 \%$ and $20 \%$ for thin clouds. In addition, the regime for cloud thickness under which we observe an enhanced exchange is restricted and short-lived. As a consequence, we observe a smaller area over which the enhanced evapotranspiration and carbon uptake are present (a maximum of $8 \%$ and $13 \%$ of the whole surface, respectively). In addition, the area under which latent heat flux and carbon uptake are reduced is comparable or larger than the enhanced area, especially in the afternoon, thus compensating the effect of thin clouds by thick clouds. All factors 
combined limit the sensitivity of domain-averaged boundary-layer and cloud dynamics to direct and diffuse radiation.

We extend our study by performing sensitivity analysis to various canopy conditions (LAI), and atmospheric conditions (background diffuse radiation). It is found that an increase in LAI, in our study from 2 to 5 , raises evapotranspiration thus lowering boundary layer and cloud base height, and slightly increasing the water content on clouds. However, the increase in LAI does not change the sensitivity of the canopy to cloud optical properties. In the experiment accounting for background diffuse radiation, the enhanced activity under thin clouds is dampened, but the main features of the reference experiment remain.

Our findings invite to be investigated under a wider range of meteorological and plant conditions. The response of vegetation is very likely to change under water-stress conditions or different temperature or water content in the atmosphere. A more realistic behavior of plants would include a gradual response of stomata to changing light conditions, which may affect the surface fluxes. Further research should include the variations in longwave radiation due to clouds, as they may lie in the order of the enhanced and decreased surface fluxes due to shading. In addition, a study of the shaded surface, paying attention to local vertical motions, could provide a more complete understanding on the local effects of diffuse radiation in the boundary layer. Numerical experiments with background wind or a more realistic (non-instantaneous) light response of vegetation would contribute to the understanding on the role of diffuse radiation. An assessment of the sensitivity of our results to the leaf angle distribution in the plant physiological model would be of interest for a better understanding of the role of the canopy in the system. Furthermore, the low cloud cover characteristic of shallow cumulus situations (maximum of $20 \%$ and restricted to less than 3 hours in this study) limits the impact of cloud-dependent surface fluxes on the boundary layer. Thus, different results are possible on more sensitive cases, such as a boundary layer topped by stratocumulus. The optical depths of stratocumulus are usually constrained to $\tau<20$ and do not reach locally as high values as those for certain shallow cumulus, while the cloud cover can be much larger than in our study. Although in stratocumulus clouds the updrafts are not the trigger for clouds, they do play a role in the evolution of the cloud layer and its break up. Thus, diffuse and direct radiation partition may be relevant in the evolution of a stratocumulus-topped boundary layer. Finally, our results, located in mid-latitudes, may differ from those closer to the equator with smaller zenith angles and, thus, larger amounts of diffuse radiation for thin clouds, as well as a wider range of cloud optical depths with significant diffuse radiation. 


\section{Acknowledgments}

The authors acknowledge the valuable comments and suggestions by three anonymous reviewers. The numerical simulations were performed with the supercomputer facilities at SURFsara and sponsored by the project NWO Physical Science Division SH-312-15. This study was supported by the grant from the Netherlands Organization for Scientific Research : NWO ALW Open Programme (2105/08597/ALW). The main conclusions of this study were presented at an international conference thanks to the partial funding by Sticthing LEB Foundation. 



\section{The diurnal stratocumulus-to- cumulus transition over land}

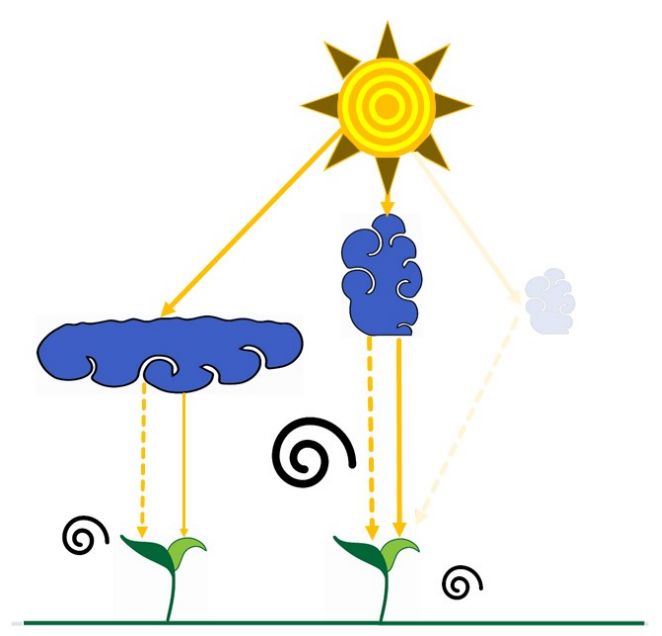

This chapter is conditionally accepted for publication and available at:

Pedruzo-Bagazgoitia, X., de Roode, S. R., Adler, B., Babić, K., Dione, C., Kalthoff, N., Lohou, F., Lothon, M., and Vilà-Guerau de Arellano, J. (2019b). The diurnal stratocumulus-to-cumulus transition over land. Atmospheric Chemistry and Physics Discussions, 2019:1-30 


\section{Abstract}

The misrepresentation of the diurnal cycle of boundary-layer clouds by large scale models strongly impacts the modeled regional energy balance in southern West Africa. In particular, recognizing the processes involved in the maintenance and transition of the nighttime stratocumulus to diurnal shallow cumulus over land remains a challenge. This is due to the fact that over vegetation, surface fluxes exhibit a much larger magnitude and variability than on the more researched marine stratocumulus transitions. An improved understanding of the interactions between surface and atmosphere is thus necessary to improve its representation. To this end, the DACCIWA measurement campaign gathered a unique dataset of observations of the frequent stratocumulus to cumulus transition in southern West Africa. Inspired and constrained by these observations, we perform a series of numerical experiments using Large Eddy Simulation. The experiments include interactive radiation and surface schemes where we explicitly resolve, quantify and describe the physical processes driving such transition. Focusing on the local processes, we quantify the transition in terms of dynamics, radiation, cloud properties, surface processes and the evolution of dynamically relevant layers such as subcloud layer, cloud layer and inversion layer. We further quantify the processes driving the stratocumulus thinning and the subsequent transition initiation by using a liquid water path budget. Finally, we study the impact of mean wind and wind shear at cloud top through two additional numerical experiments. We find that the sequence starts with a nighttime well-mixed layer from surface to cloud top, in terms of temperature and humidity, and transitions to a prototypical convective boundary layer by the afternoon. We identify radiative cooling as the largest factor for the maintenance leading to a net thickening of the cloud layer of about $18 \mathrm{~g} \mathrm{~m}^{-2} \mathrm{~h}^{-1}$ before sunrise. Four hours after sunrise, the cloud layer decouples from the surface through a growing negative buoyancy flux at cloud base. After sunrise, the increasing impact of entrainment leads to a progressive thinning of the cloud layer. While the effect of wind on the stratocumulus layer during nighttime is limited, after sunrise we find shear at cloud top to have the largest impact: the local turbulence generated by shear enhances the boundary layer growth and entrainment aided by the increased surface fluxes. As a consequence wind shear at cloud top accelerates the breakup and transition by about 2 hours. The quantification of the transition and its driving factors presented here sets the path for an improved representation by larger scale models. 


\subsection{Introduction}

Stratocumulus (Sc) clouds play a critical role on the radiative balance of the planet given their high albedo (Hartmann et al., 1992; Chen et al., 2000) and extensive cover worldwide (Eastman and Warren, 2014; Eastman et al., 2014). These boundary-layer clouds are a common feature in the southern West Africa (SWA), and recur in the night and morning during the Monsoon season between May and September (van der Linden et al., 2015; Hill et al., 2018). Possible future changes in highly sensitive Sc forcings in SWA, such as anthropogenic regional aerosol increase (Boucher et al., 2013) or the global $\mathrm{CO}_{2}$ rise (Schneider et al., 2019), further motivate a better understanding of the boundary-layer cloud dynamics over land in SWA.

During the monsoon season the intertropical convergence zone shifts northward till $15^{\circ}$ $\mathrm{N}$, facilitating the extension of the maritime air masses inland. The arrival of the cooler, but not necessarily moister, mass of air more than a $100 \mathrm{~km}$ inland facilitates the onset of Sc clouds over land (Adler et al., 2019; Babić et al., 2019; Dione et al., 2019). The fact that this mass of air is characterized by cloudless conditions when over the sea reveals the importance of the land and other local factors on the cloud formation and maintenance (Adler et al., 2019; Babić et al., 2019; Lohou et al., 2019). Lohou et al. (2019) extended the previous work and summarized the four phases leading from cloud formation to dissipation. In addition, they described three observed scenarios for the breakup and dissipation of the Sc deck along the day. Such scenarios differed on the Sc coupling to surface and on the presence of convective clouds below the Sc.

The high albedo of low Sc clouds and its underestimation by most climate models lead to significant biases on the regional surface energy balance if the evolution and spatial structure of the cloud field is not correctly represented (Hannak et al., 2017). More specifically, the maintenance, dissipation or transition to other cloud forms of the Sc cloud layer after sunrise has strong implications in the regional energy balance (Knippertz et al., 2011; Hannak et al., 2017; Lohou et al., 2019). To improve our understanding and better quantify the effects of Sc clouds over land in a observation-scarce region, the Dynamics-aerosol-chemistry-cloud interactions in West Africa (DACCIWA) project deployed an extensive network of observations during June and July in 2016 comprising three fully instrumented supersites (Knippertz et al., 2015; Flamant et al., 2018; Kalthoff et al., 2018). The resultant dataset of high spatio-temporal observations of the cloud transition allows us to tackle two important questions. Firstly, it allows us to understand the transition (Lohou et al., 2019) and, using idealized numerical simulations, reproduce a characteristic stratocumulus to cumulus $(\mathrm{Sc}-\mathrm{Cu})$ transition with typical conditions of SWA. Secondly, we aim at identifying the physical processes and their complex interplay that leads to a breaking up of the Sc deck.

Here, we extend on the impacts of the land-atmosphere interactions on the Sc-Cu transi- 
tion and breakup. Previous studies have largely relied on high resolution explicit modeling, e.g. Large Eddy Simulation (LES), of marine Sc clouds. Over sea, surface fluxes are low and show little diurnal variation. Evaporation from the sea provides the necessary moisture to maintain the Sc layer, that is well-mixed down to the surface by the turbulence generated at the cloud top by radiative cooling (Wood, 2012). Transitions from Sc to shallow cumulus have also been studied through LES mostly in maritime conditions (Bretherton et al., 1999a; Sandu and Stevens, 2011; de Roode et al., 2016). Such transitions are typically investigated using a Lagrangian approach in which the trajectory of an air mass is followed as it is advected from the subtropics towards the equator. An increasing sea surface temperature and decreasing subsidence is usually imposed along the trajectory, leading to increasing latent heat fluxes and boundary layer height, respectively. The main mechanism for such transitions over sea is the increase in buoyancy along the cloud layer by higher latent heat flux, leading to larger entrainment velocities aided by the subsidence decrease, and the eventual dissipation of the Sc cloud layer. Over land, however, such transitions may have different drivers, given their differently partitioned surface fluxes as well as their larger magnitude and diurnal variability than over sea. Ghonima et al. (2016) performed a thorough idealized LES study on Sc-Cu transitions both over land and over sea. They based all their cases on vertical profiles of mid-latitude marine conditions and prescribed different Bowen ratios to regulate the surface fluxes over land. In contrast, atmospheric conditions in near-equatorial SWA are characterized by a moister and warmer atmosphere as well as stronger solar irradiation and, locally, larger evapotranspiration. These differences pose the question of whether the mechanisms and physical processes identified by Ghonima et al. (2016) remain relevant for SWA. Our study thus aims at filling the knowledge gap on turbulence resolving numerical experiments of $\mathrm{Sc}-\mathrm{Cu}$ transitions taking place over land and, specifically, in sub-tropical atmospheric conditions. We systematically focus on the following processes and the role played in the maintenance of the Sc and its transition to cumulus clouds: radiation, entrainment and the land surface fluxes. Radiation is the source for cloud maintenance during night and, as the day evolves, a factor for dissipation. Entrainment is known to affect the cloud layer by drying and warming it, rising it and weakening the thermal inversion. The land surface fluxes respond to variations in wind and radiation and affect the transport of heat and moisture to the cloud layer as well as the entrainment. In addition, we briefly study the evolution of metrics frequently used by parameterizations in larger scale models along the $\mathrm{Sc}-\mathrm{Cu}$ transition .

Finally, during the DACCIWA campaign a recurrent low level jet along the cloud layer was observed (Adler et al., 2019; Dione et al., 2019), raising an additional question on the effects of wind shear on Sc and its transition d(Lohou et al., 2019). Previous work on modeled sheared Sc over sea suggests that shear at cloud top lowers the water content of Sc by enhancing the entrainment rate (Wang et al., 2008, 2012). Mechem et al. (2010) presented a land Sc case and briefly studied the effects of shear. They similarly concluded 
that entrainment velocity increases, leading to a decrease in cloud liquid water content in presence of cloud-top wind shear. However, to the best of our knowledge, there is no work studying the effects of wind shear on stratocumulus to cumulus transitions. Thus, we additionally perform some sensitivity studies on the effect of wind and wind shear at cloud top on the Sc-Cu transition.

Our research seeks to answer these three research questions:

- How is a stratocumulus to cumulus transition over land characterized? What is the relevance of the local processes?

- How do the contributions of each physical process vary with time during the maintenance, thinning and transition of the cloud layer?

- How do metrics relevant for larger scale models quantify the transition?

\subsection{Methods}

\subsubsection{Dutch Atmospheric Large Eddy simulation (DALES)}

To explicitly resolve the $\mathrm{Sc}-\mathrm{Cu}$ transition we perform our numerical experiments using the Dutch Atmospheric Large Eddy Simulation (DALES) (Heus et al., 2010; Ouwersloot et al., 2016). LES models explicitly resolve most of the energy-containing turbulent motions in the boundary layer, including the stratocumulus and shallow cumulus cloud dynamics. Based on the initial work of Nieuwstadt and Brost (1986), DALES is a LES model that has been used individually or within a model intercomparison for a broad range of cases, from clear sky diurnal cycles (Pino et al., 2003) to boundary layers topped by stratocumulus (Blossey et al., 2013; van der Dussen et al., 2015) or cumulus (Siebesma et al., 2003; VilàGuerau de Arellano et al., 2014), including Sc-Cu transitions over sea (van der Dussen et al., 2013; de Roode et al., 2016). Here, we use the DALES 4.1 version. We describe below the relevant parameterizations for this study.

- An interactive land surface model with a mechanistic representation of plant behavior. It regulates the surface latent, and sensible heat fluxes, as well as the $\mathrm{CO}_{2}$ flux depending on environmental variables such as $\mathrm{CO}_{2}$ concentration, atmospheric vapor pressure deficit, temperature, soil moisture and surface wind (Jacobs and de Bruin, 1997; van Heerwaarden et al., 2010; Vilà-Guerau de Arellano et al., 2015). It is upgraded with a 2 big-leaf (sunlit and shaded leaves) scheme allowing for different penetration rates of direct and diffuse radiation along the canopy (Pedruzo-Bagazgoitia et al., 2017). The fact that surface fluxes are higher and more variable over land, responding to environmental variables and potentially altering 
the boundary layer and cloud structure, explains the need for an interactive scheme as the one presented here.

- The two-stream radiation scheme RRTMG (Iacono et al., 2008). It is used to provide longwave and direct and diffuse shortwave radiation at each gridbox dependent on liquid water and other chemical compounds.

- The microphysics scheme by Khairoutdinov and Kogan (2000), specifically designed for Sc clouds. It includes cloud-droplet sedimentation, found to be highly relevant in the representation of Sc clouds (Ackerman et al., 2004; Bretherton et al., 2007; Dearden et al., 2018).

\subsubsection{Observations}

We base our idealized study on observations taken during the field campaign of the DACCIWA project during the months of June and July 2016. We focus on the observations of 26th June 2016 at the Savé supersite. On this day a stratocumulus deck was observed during the night and morning above Savé, followed by a cloud base rise and breakup during the late morning and afternoon (Dione et al., 2019). We briefly describe below the methods and observations used to inspire our idealized study. For a fully detailed explanation of the observations and the dataset, the reader is referred to Kalthoff et al. (2018) and Bessardon et al. (2019), respectively.

- Radiosondes were performed with the MODEM radiosounding system. The temperature and relative humidity of the air were measured with a 1 second temporal resolution ( $\simeq 4-5 \mathrm{~m}$ in vertical resolution). The wind speed, direction and the pressure were determined based on the radiosonde GPS coordinates (Derrien et al., 2016).

- The cloud base height is measured by a continuously running ceilometer measuring backscatter profiles with a 1 minute resolution. From backscatter profiles a cloud base height is obtained using the manufacturer algorithm. The data is available at Handwerker et al. (2016).

- The cloud top height is measured by a dual-polarized cloud radar, which allows to distinguish between hydrometeors and other targets. The cloud top height is estimated from the 5 min averaged reflectivity profiles of hydrometeors applying a threshold of $-35 \mathrm{dBz}$ (Bauer-Pfundstein and Goersdorf, 2007). Therefore, reflectivities larger than $-35 \mathrm{dBz}$ are considered clouds. The data is available at Handwerker et al. (2016).

- The cloud cover is calculated as the percentage of cloud base height measurements below 1000 m (Adler et al., 2019; Zouzoua, 2019). The values are averaged over 19 

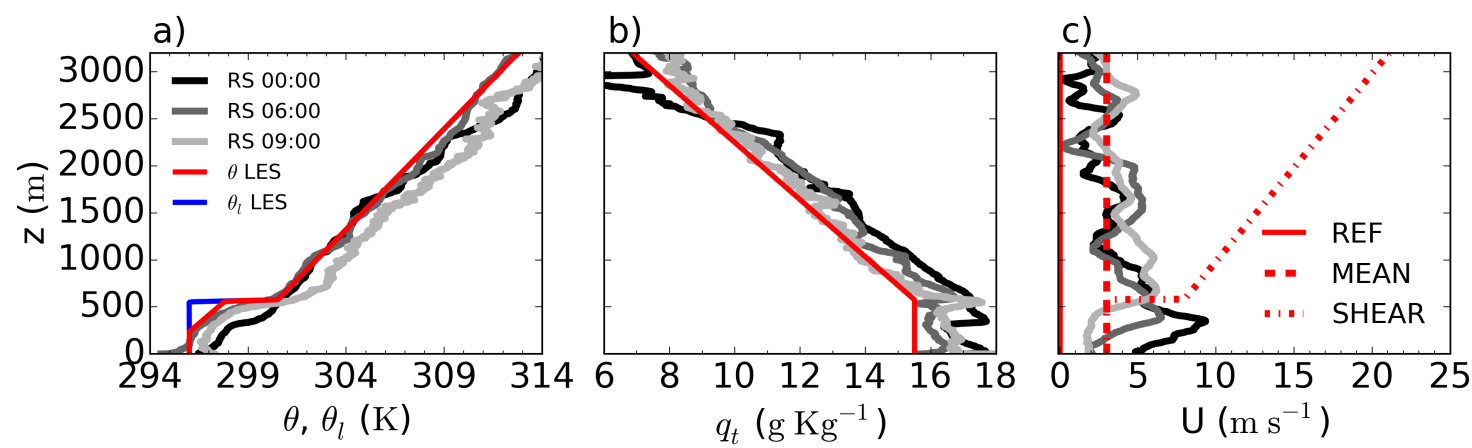

Figure 4.1: Vertical profiles of potential temperature (a), total specific humidity (b) and wind (c) as observed through radiosondes (at 00 00, 0600 and 0900 UTC from black to light grey) on 26th June 2016 and as prescribed in the idealized LES experiments (red and blue). The three experiments REF, MEAN and SHEAR differ only in the prescribed wind profiles.

days during the campaign to prevent too high variability by single point observations of individual days.

- The surface fluxes are obtained from an energy balance station deployed over a mix of grass and bushes. The 30-min turbulent fluxes are calculated from highfrequency (20 Hz sampling rate) measurements of wind speed and sonic temperature obtained by ultrasonic anemometer and humidity measurements which are based on the absorption of near-infrared radiation and obtained by the fast-response LI-COR sensor by applying the eddy-correlation method (Mauder et al., 2013). The data is available at Kohler et al. (2016).

- The two sets of turbulent kinetic energy measurements are calculated from the anemometer measurements of wind speed at $4 \mathrm{~m}$ and $7.8 \mathrm{~m}$ by two energy balance stations deployed over a mix of grass and bushes and over corn, respectively.

\subsubsection{Model settings and initial conditions}

Constrained by the surface and upper atmospheric observations, we design an academic case to be simulated through LES. Our aim is, by means of an idealized numerical experiment, to simulate a Sc-Cu transition, including the Sc breakup, during typical atmospheric conditions in SWA rather than the reproduction of an exact day occurred during the DACCIWA measurement campaign. In particular, we study the Sc-Cu transition of a coupled case as described by Lohou et al. (2019).

We design a $12 \times 12 \mathrm{~km}^{2}$ wide and $3.2 \mathrm{~km}$ high domain, with a gridbox size of $50 \times 50 \times 4 \mathrm{~m}^{3}$ resulting in 800 vertical levels. Such high vertical resolution is required in order to reduce the overestimation of mixing and entrainment typical of coarser LES simulations with Sc (Bretherton et al., 1999b; Stevens et al., 2005). Although at this vertical resolution 
processes such as evaporative cooling and cloud top mixing might still be overestimated (Stevens et al., 2005; Mellado, 2017), a much finer resolution, or a Direct Numerical Simulation approach, would not allow computationally for an integrated simulation of both cloud top and surface. As it will be shown later, both interfaces play a critical role in the development and transition studied here. We use periodic boundary conditions on the horizontal directions. We start the experiment at 330 UTC to allow for one hour of spin up of the Sc layer and end the experiment at 1830 UTC after sunset.

We prescribe a subsidence profile, following Bellon and Stevens (2012), of the shape $w_{\text {subs }}(z)=-w_{0}\left(1-e^{\frac{-z}{z w}}\right)$, with $w_{0}=5.3 \mathrm{~mm} \mathrm{~s}^{-1}$ and $z_{w}=300 \mathrm{~m}$. Such a profile translates to $w_{\text {subs }}=-4.51 \mathrm{~mm} \mathrm{~s}^{-1}$ at the initial cloud top height of $570 \mathrm{~m}$. Our choice for the subsidence profile is justified given the uncertainty and high temporal variability in subsidence profiles, as well as its large spread among regional simulations carried out with the Consortium for Small-Scale Modeling (COSMO) within the DACCIWA project or ERA-interim reanalysis. To limit the complexity of our idealized experiments and focus on the interaction of the surface and boundary-layer processes, we prescribe no advection of heat or moisture at any height. Adler et al. (2019) and Babić et al. (2019) found cold air advection necessary for the formation of the cloud layer. Yet its relevance decreased as sunrise approached, thus justifying our assumption during our time of interest.

For all the experiments we calculate the vertical profiles of the radiative fluxes every minute. In doing so, we quantify how radiative fluxes are perturbed by the liquid water related to cloud dynamics and how they interact with the surface. This is done to account for fast fluctuations of net radiation at cloud top and surface. The latter is relevant given its potential to alter surface fluxes and, thus, the evolution of the boundary layer and clouds (Vilà-Guerau de Arellano et al., 2014; Gronemeier et al., 2016; Sikma and Vilà-Guerau de Arellano, 2019). Based on aircraft observations during the DACCIWA campaign (Taylor et al., 2019), the cloud droplet number concentration is set to $300 \mathrm{~cm}^{-3}$ and remains constant throughout the experiment.

We show in Fig.4.1 the vertical profiles obtained through three radiosondes during the night and morning of $26^{\text {th }}$ June 2016. The radiosonde at 600 UTC, the closest to our initialization time, shows a strong increase in potential temperature of about $3 \mathrm{~K}$ at 570 meters high. Above, all radiosondes show similar temperature lapse rates of about $4.6 \mathrm{~K} \mathrm{~km}^{-1}$. Subtropical marine Sc clouds are frequently capped by a strong drying above cloud top (Duynkerke et al., 2004; Wood, 2012). Yet none of the radiosonde profiles show any strong drying above 570 meters. If any, they show a humidity increase above cloud layer. Such increase could be related to the previously questioned reliability of radiosonde measurements as they exit the cloud layer through their ascension (Lorenc et al., 1996; Mechem et al., 2010; Babić et al., 2019). Situations without a dry jump above Sc cloud top have been previously reported over land (Mechem et al., 2010) and are more typical of arctic climates (Morrison et al., 2012). 
The observations demonstrate that the idealized experiment's initial conditions lie within typical meteorological conditions in SWA. The initial idealized profiles prescribe a well mixed layer up to 570 meters with liquid-water potential temperature $\theta_{l}=296 \mathrm{~K}$ and specific humidity $q_{t}=15.5 \mathrm{~g} \mathrm{~kg}^{-1}$. Such thermodynamic conditions result in a domaincovering cloud layer from $226 \mathrm{~m}$ to $570 \mathrm{~m}$ high, topped by a jump of $4.5 \mathrm{~K}$ in temperature, but without a jump in specific humidity. Above $570 \mathrm{~m}$ the potential temperature and total moisture idealized profiles exhibit constant slopes of $4.67 \mathrm{~K} \mathrm{~km}^{-1}$ and $3.29 \mathrm{~g} \mathrm{~kg} \mathrm{k}^{-1} \mathrm{~km}^{-1}$ respectively. Given the spread in vertical profiles by radiosondes, we performed additional simulations exploring variations in the profiles of $0.5 \mathrm{~K}$ and $0.5 \mathrm{~g} \mathrm{~kg}^{-1}$. Results showed very similar development of the Sc-Cu transition.

Our reference experiment REF prescribes no wind at all heights. To study the effect of wind and wind shear, we perform two additional numerical experiments, MEAN and SHEAR, where we account for different idealized wind effects. This sensitivity analysis is motivated by the recurrent winds with the shape of low-level jet, such as those in Fig. 4.1c, that were frequently observed during the DACCIWA campaign (Kalthoff et al., 2018; Adler et al., 2019; Dione et al., 2019). Failed attempts to maintain a low level jet-like wind profile together with the Sc cloud layer in preliminary experiments suggest that the jet-like wind is the result of large scale dynamics, and, thus, beyond the scope of the present study on local factors. The large scale origin of the low level wind is also supported by more detailed observational analysis (Babić et al., 2019; Adler et al., 2019; Dione et al., 2019). Following our idealized approach, the initial wind speed and wind direction are inspired by the observations and adapted to better study how these effects influence the $\mathrm{Sc}-\mathrm{Cu}$ transition. In this case, the mean wind and the shear at the cloud top are considered.

We prescribe a constant horizontal wind of $3 \mathrm{~m} \mathrm{~s}^{-1}$ along the whole vertical profile in MEAN based on above cloud layer radiosonde observations (Fig.4.1c). Consistent with our idealized setting, we assume the wind to blow only along the $\mathrm{x}$-direction and without prescribed directional shifts with height. In SHEAR we add a jump of $5 \mathrm{~m} \mathrm{~s}^{-1}$ to the mean $3 \mathrm{~m} \mathrm{~s}^{-1}$ at cloud top to represent a wind shear of similar magnitude as the observed low level jet. The values prescribed here for the simplified effects of the low level jet are representative not only of the day studied here but also of the whole measurement campaign (Dione et al., 2019). The free troposphere wind shows a constant increase of $5 \mathrm{~m} \mathrm{~s}^{-1} \mathrm{~km}^{-1}$ in SHEAR. Our aim here is to maintain a shear contribution as the cloud layer rises. We prescribe geostrophic winds identical to the initial wind profiles, as the goal is to observe the impact of wind on the transition and not vice versa. In summary, differences between MEAN and REF serve in identifying the role played by a mean wind, which will mainly enhance the surface fluxes. MEAN and SHEAR differences show the impact of the local shear at cloud top. 


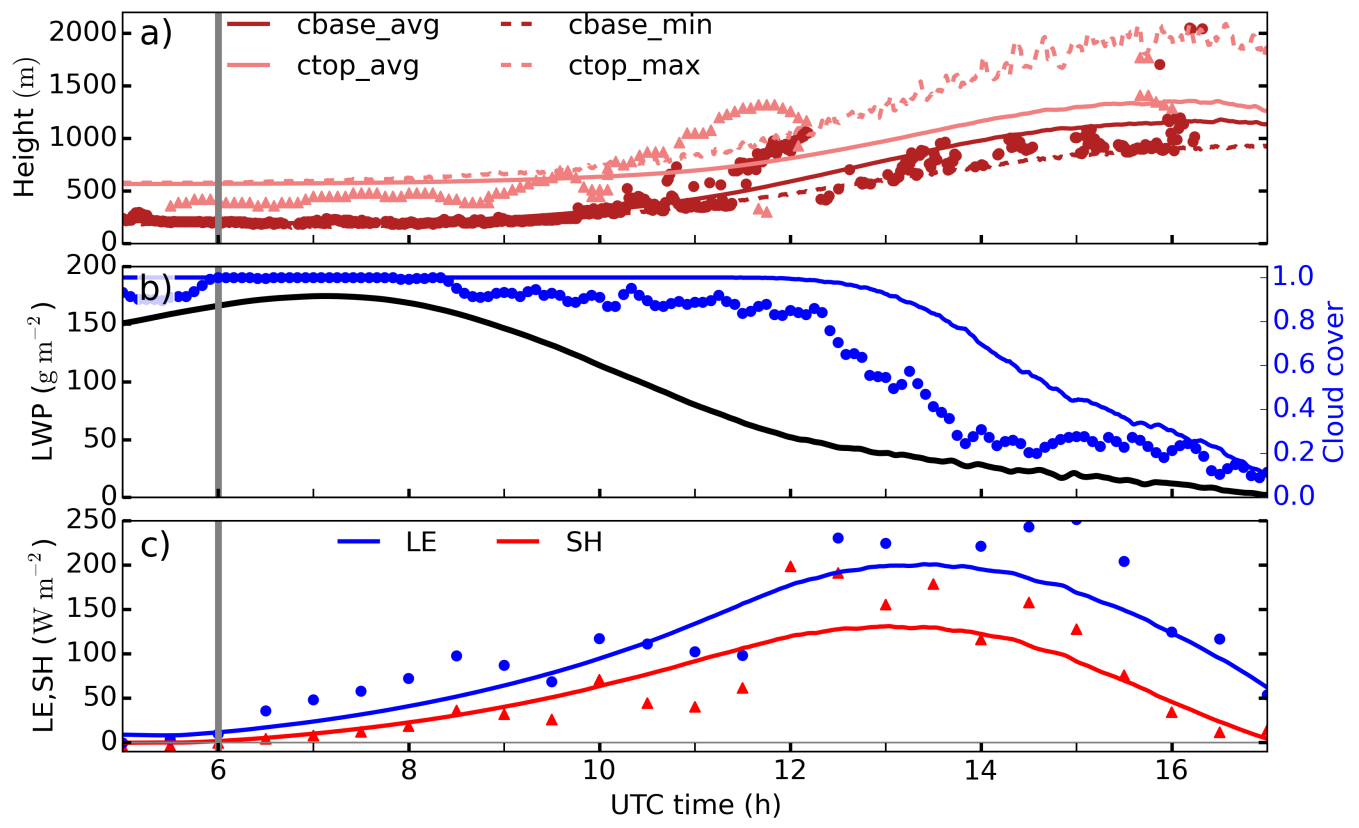

Figure 4.2: Time series of the domain average cloud base (cbase_avg in full dark red line) and cloud top (ctop_avg in full light red line), maximum cloud top (ctop_max in dashed light red line) and minimum cloud base (cbase_min in dashed dark red line) (a), liquid water path and cloud cover (b) and latent and sensible heat fluxes (c) in REF. Observed cloud base and cloud top heights on 26th June are represented by dark red circles and light red triangles, respectively, in (a). Observed cloud cover, averaged over 19 campaign days is shown in blue circles in (b). Observed latent and sensible heat fluxes are shown by blue circles and red triangles, respectively, in (c). The vertical grey lines indicate the sunrise time.

\subsection{Results}

\subsubsection{Evolution of the transition}

Figure 4.2 shows the diurnal evolution of cloud height, cover, and liquid water path (LWP) and their connection with surface turbulent fluxes in the REF simulation. It also includes the observations corresponding to the day by which our case is inspired. At Fig. 4.2a both cloud top and base remain approximately constant for the first hours. The LWP values are on the high end of domain average LWP for marine stratocumulus cloud (Wood, 2012) and coincide with observed ones during the DACCIWA campaign (Babic et al., 2019; Kalthoff et al., 2018). The initially constant cloud top height coincides with the boundary layer height. As a result, the boundary layer height evolution can be expressed using mixed layer theory by the relation that equates the entrainment velocity and the 
subsidence. It reads, assuming horizontally homogeneous conditions (Lilly, 1968):

$$
\frac{\partial h}{\partial t}=w_{e}+w_{\text {subs }}(h) \simeq 0
$$

with $h$ the boundary layer height defined as the height of minimum buoyancy flux, $w_{e}$ the entrainment velocity and $w_{\text {subs }}(h)$ the subsidence, depending on height as described in Sec.4.2.3, at $h$. For this experiment and before sunrise $w_{e} \simeq-w_{\text {subs }}(h)=0.45 \mathrm{~cm} \mathrm{~s}^{-1}$, which is in the same order of magnitude as previously reported nocturnal marine Sc cases (Stevens et al., 2003). Between 2 to 3 hours after sunrise (6 00 UTC) the cloud layer begins to rise and subsequently decreases its water content. Along this time the domain average cloud base cbase_avg follows the observed cloud base, and so do the surface fluxes with the observed ones. The onset of the convective phase, defined by Lohou et al. (2019) as the time when the sensible heat flux $S H>10 \mathrm{~W} \mathrm{~m}^{-2}$, takes place between 700 and 730 UTC according to observations and at 655 UTC in REF. The breakup in the cloud layer, defined as the time when cloud cover $(c c)$ is below 1, takes place at around 1130 UTC in our experiment and coincides with the observed sharp increase in surface fluxes of about $150 \mathrm{~W} \mathrm{~m}^{-2}$, i.e., a threefold increase compared to before-breakup values. This sudden change reveals that surface fluxes are radiation-driven. The good agreement in the surface flux partitioning as modeled and observed justifies the use of a land surface model sensitive to several environmental variables at surface (see Sec. 4.2.1). After 11 30 UTC observations show large variability in measured cloud base heights (Fig. 4.2a), suggesting either the presence of shallow clouds below the Sc cloud layer or the breakup of Sc layer (Lohou et al., 2019). After cloud break up, cc decreases quasi-linearly until the end of the simulation. The same pattern for cloud cover is shown by observations, although one hour in advance. Note, however, that the observations of $c c$ are averaged over 19 days timeseries selected due to a cloud onset before 400 UTC (Zouzoua, 2019). The variability between the days considered in the average also explains the $c c$ values below 1 before 600 UTC and after 830 UTC.

\subsubsection{Transition on turbulence and radiation}

The turbulent spatial structure explaining this transition from typical nocturnal stratocumulus to convective clouds is shown in Figure 4.3 through the buoyancy flux and temperature profiles. The initial stages of the LES experiment (Fig. 4.3a,b) present a well mixed and fully coupled layer from surface to cloud top. This layer is limited by a strong jump in liquid water potential temperature $\theta_{l}$ of about $4 \mathrm{~K}$ at 500 UTC (Fig. 4.3 a) at around $550 \mathrm{~m}$, and a very thin inversion layer. We quantify this layer through their lower and upper limits $z i^{-}$and $z i^{+}$, respectively. These heights are defined, following van der Dussen et al. (2016), as the heights above and below, respectively, the maximum

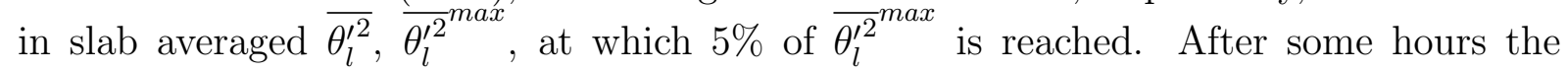
boundary layer evolves to a well mixed subcloud layer with a conditionally unstable cloud 


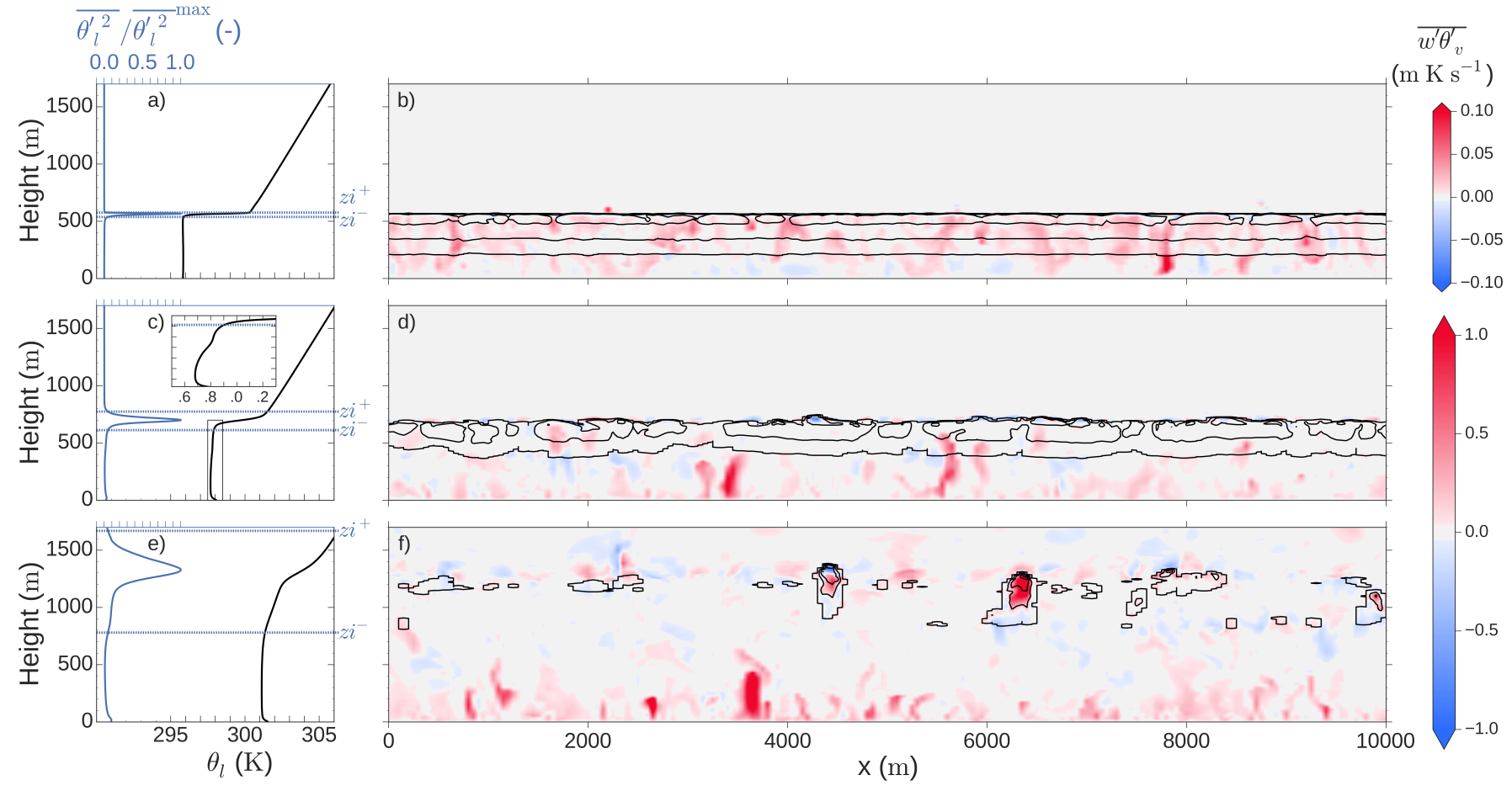

Figure 4.3: On the left, slab averaged vertical profile of liquid potential temperature $\theta_{l}$ (black) and $\overline{\theta_{l}^{\prime 2}}$ normalized over its maximum value (blue). The inversion layer upper $z i^{+}$ and lower $z i^{-}$limits are indicated in dashed blue horizontal lines. On the right, horizontal cross-section of buoyancy flux $\overline{w^{\prime} \theta_{v}^{\prime}}$ (in colours, red (blue) indicating upwards (downwards) movement of buoyantly positive (negative) air) and cloud liquid water (in black contour lines every $0.3 \mathrm{~g}_{\mathrm{w}} \mathrm{Kg}_{\mathrm{a}}^{-1}$ ). Top plot corresponds to $500 \mathrm{UTC}$, center to $1100 \mathrm{UTC}$ and bottom to 1430 UTC. The inset at c) is an expanded version of the rectangle in the same subfigure.

layer aloft at 1430 UTC and a very broad inversion layer. Such evolution of the inversion layer allows us to interpret the typically conditionally unstable region of the cloudy layer in convective conditions as an expanded analogue of the very sharp inversion layer in Sc clouds. Thus, to correctly represent the transition studied here it is necessary to treat the evolution as a transition where the inversion layer expands as the boundary layer grows. A more detailed evolution of the inversion layer is given in Fig. 4.5.

In the absence of mechanical production of turbulence, buoyancy is the only driving mechanism for turbulence. Figs $4.3 \mathrm{~b}, \mathrm{~d}$,f quantify the shift of buoyancy-driven turbulence generation from cloud top radiative cooling at 500 UTC to surface warming at 1430 UTC. Note the change in scale by a factor of 10 in $\overline{w^{\prime} \theta_{v}^{\prime}}$ between Fig $4.3 \mathrm{~b}$ and $4.3 \mathrm{~d}$. Such a difference in magnitude shows that the surface-driven turbulence after sunrise becomes stronger, about 10 times, than the one created by cloud-top cooling. In fact, the cloudtop cooling contribution to the buoyancy flux is in part diminished by a compensating condensational warming within the cloud layer. At 1100 UTC there is a critical moment in the transition: the cloud layer remains rather homogeneous, but the mixed layer is 

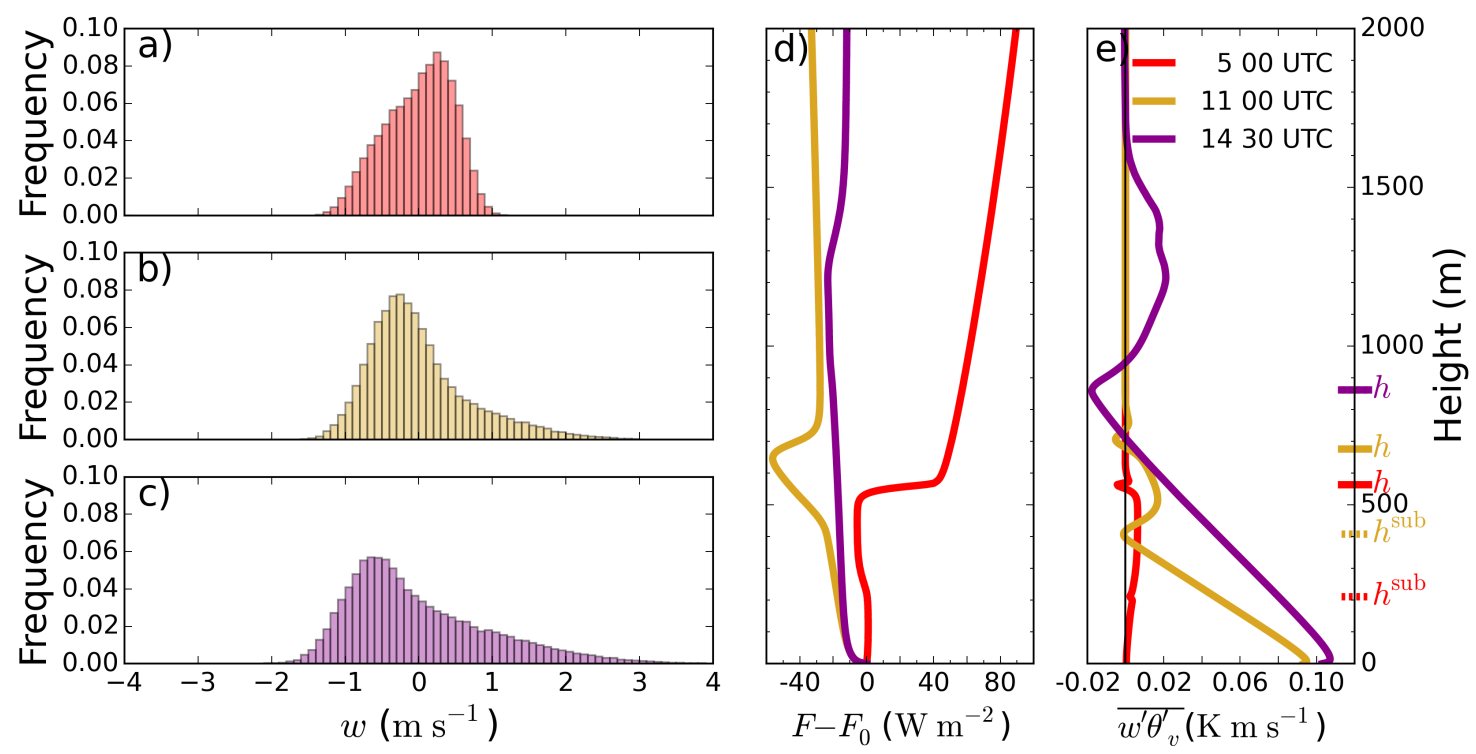

Figure 4.4: On the left, frequency distribution of vertical velocities at $\frac{h}{2}$ at 500 UTC (a), 1100 UTC (b) and 1430 UTC (c). On the center (d), vertical profile of slab net radiative flux normalized over the surface value at 500 UTC (red), 1100 UTC (dark yellow) and 1430 UTC (purple). On the right (e) and following the same color code, slab averaged buoyancy flux $\overline{w^{\prime} \theta_{v}^{\prime}}$. The subcloud layer height $h^{\text {sub }}$ and the boundary layer height $h$ are shown for each time at the right vertical axis in (e). At 1430 UTC both heights coincide.

now simultaneously driven both by surface warming and cloud top cooling. As it will be shown later (Figs. 4.4 and 4.6), the penetration of shortwave radiation through the cloud layer down to the surface is key in regulating both phenomena. The warming of the cloud layer leads to a decoupling of the cloud and subcloud layers. This is already visible at 11 $00 \mathrm{UTC}$ with a temperature difference between layers of about $0.2 \mathrm{~K}$ at $400 \mathrm{~m}$ high (see inset in Fig. 4.3c).

By resolving interactively the radiation transfer along the cloud layer and the surface response we gain insight on the dynamical transition, as shown in Figure 4.4. There, we observe how the vertical velocity distribution at the middle of the boundary layer starts from a situation with limited extreme velocities (between -1.3 and $1 \mathrm{~m} \mathrm{~s}^{-1}$ ) and a negative skewness of $S_{w}=-0.3$ at 500 UTC, where $S_{w}=\frac{\overline{w^{3}}}{\overline{w^{2} \frac{3}{2}}}$. This value for $S_{w}$ lies within the limits of typical marine Sc clouds (Ghate et al., 2014). It then evolves to a prototypical convective-boundary-layer (CBL) skewed distribution with a larger spread of vertical velocities at $1100 \mathrm{UTC}$ (between -1.5 and $2.7 \mathrm{~m} \mathrm{~s}^{-1}$ ) and $S_{w}=1.2$ at half of the boundary layer height, having skewness values typical of dry convective boundary layers (Lenschow et al., 2012) or situations with cumulus coupled to Sc clouds (de Roode and Duynkerke, 1996). Similar values for $S_{w}$ are found at 1430 UTC, with minimum and maximum vertical velocities between -1.8 and $3.5 \mathrm{~m} \mathrm{~s}^{-1}$, respectively. The transition from stratocumulus to prototypical convective conditions is reinforced by the evolution of 
the radiative profiles. Figure $4.4 \mathrm{~d}$ shows an initial net radiative divergence at the cloud top of $43 \mathrm{~W} \mathrm{~m}^{-2}$. The related cooling drives the mixed layer at $500 \mathrm{UTC}$. At this time the radiative cooling is stronger than the warming by entrainment as the mixed layer cools at a rate of about $0.1 \mathrm{~K} \mathrm{~h}^{-1}$ before sunrise (not shown). By $1100 \mathrm{UTC}$ there is a net radiative warming along the cloud layer (between 400 and $650 \mathrm{~m}$ high, see Fig. 4.3) due to the absorption of shortwave radiation within the cloud layer. Shortwave radiation locally warms up to $1.1 \mathrm{~K} \mathrm{~h}^{-1}$ the lower two thirds of the cloud layer due to the 44 $\mathrm{W} \mathrm{m}^{-2}$ of absorbed shortwave radiation along its travel through the cloud layer (not shown). The high cloud droplet number, $300 \mathrm{~cm}^{-3}$, is likely to influence positively such net warming.

This net radiative warming along the cloud layer reinforces the warming driven by entrainment of free tropospheric air. The combination of both processes is critical for the decoupling of the cloud and subcloud layers. As it will be shown later (Fig. 4.6), it also plays a role on the thinning of the Sc and the reduction of turbulence generation at cloud top. Figure $4.4 \mathrm{e}$ shows the profile of the buoyancy flux, closely linked to the role of radiation. The averaged buoyancy flux shows a similar transition starting from prototypical nocturnal Sc clouds at 500 UTC, with positive buoyancy along the whole layer up to $550 \mathrm{~m}$ and a local minimum at cloud base due to latent heat release (Bretherton and Wyant, 1997; Wood, 2012). We define the height of such minimum as the subcloud layer height $h^{\text {sub }}$. The definition of $h^{\text {sub }}$ is necessary to better quantify the decoupling of the stratocumulus layer from the surface, as it will be shown in Fig. 4.10. At cloud top Fig. $4.4 \mathrm{e}$ presents an absolute minimum at the boundary layer height $h$. It then evolves to profiles common in cumulus topped convective boundary layers (Siebesma et al., 2003) or decoupled Sc cloud layers (Wood, 2012) with linearly decreasing $\overline{w^{\prime} \theta_{v}^{\prime}}$ up to the cloud base, and buoyantly active convective clouds above $950 \mathrm{~m}$. Note that under such conditions the boundary layer height and the subcloud top height coincide and $h^{\mathrm{sub}}=h$. The buoyancy flux profile at 1100 UTC shows the decoupling of the cloud layer from the surface by the enhancement of the local minimum at $h^{\text {sub }}$ at $400 \mathrm{~m}$. This nearly negative value in the vicinity of the cloud base has already been described as an indication of decoupling and hampered transport of moisture (and heat, in our case) from the surface to the cloud layer (Turton and Nicholls, 1987; Stevens, 2000; Lewellen and Lewellen, 2002). This will be further explored in Fig. 4.10.

We show in the time series in Fig. 4.5 the evolution of the variables that better reflect the dynamics of the Sc-Cu transition: we show the inversion layer upper and lower limits $z i^{+}$ and $z i^{-}$, respectively, the subcloud top height $h^{\text {sub }}$ and boundary layer height $h$ shown in Fig. 4.4, the maximum cloud top and minimum cloud base heights as in Fig. 4.2, and we additionally calculate the Sc cloud base cbase_Sc and cloud top ctop_Sc. These are defined as the height of the lowest and highest vertical level, respectively, with a slab averaged 


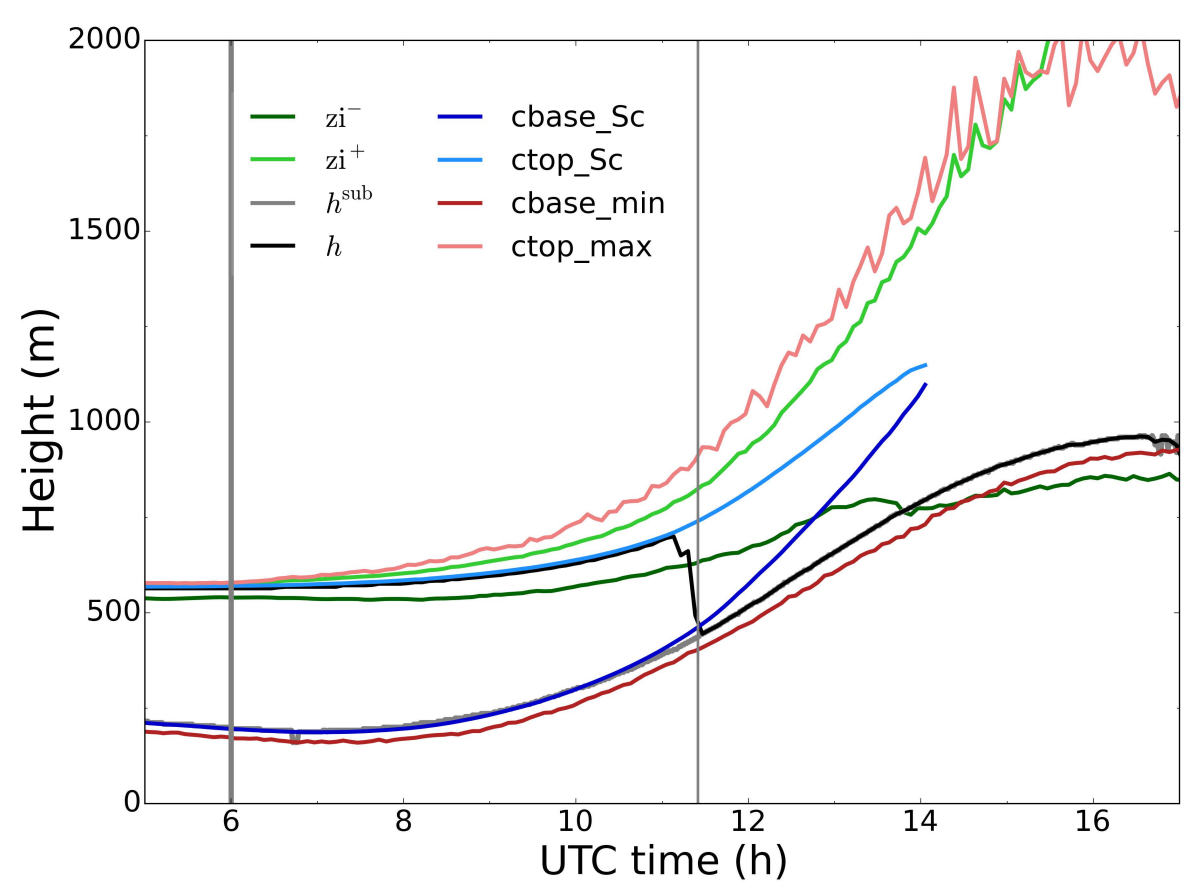

Figure 4.5: Time series of inversion layer top $z i^{+}$(light green) and bottom $z i^{-}$(dark green) heights, boundary layer height $h$ (black) and subcloud buoyancy minimum height $h^{\text {sub }}$ (grey), stratocumulus cloud base cbase_Sc (dark blue) and top ctop_Sc (light blue) heights, and minimum cloud base cbase_min (dark red) and maximum cloud top ctop_max (light red) heights. Sunrise time and cloud breakup time are indicated by the thick and thin grey lines, respectively.

cloud fraction higher than 40\%. After 1000 UTC the Sc cloud base rises faster than the minimum cloud base. This is analogous to the slower rise of Sc cloud top compared to the maximum cloud top. Due to a faster rise of Sc cloud base than Sc cloud top, there is a thinning of the Sc layer eventually dissipating at 1400 UTC. Lohou et al. (2019) observed a similar cloud thinning pattern based solely on observations. The cloud and subcloud layer dynamics divert from coupled Sc conditions, i.e. well mixed layer from surface to cloud top, several hours before, as it was shown in Figs. 4.3 and 4.4. Between 1100 and 1130 UTC, i.e. before the cloud breakup, $h$ shifts from the cloud top to the subcloud layer top represented by $h^{\text {sub }}$. The evolution of the inversion layer, indicated by $z i^{+}$and $z i^{-}$, reveals a broadening of the inversion layer from a very thin layer $(\sim 50 \mathrm{~m})$ across cloud top during the first few hours to a region thicker than $1 \mathrm{~km}$ in the afternoon due to the cumulus clouds.

\subsubsection{LWP budget before and during the transition}

After observing the transition in cloud characteristics and buoyancy regime, a question immediately arises: what is the relative contribution of the main physical processes driving 


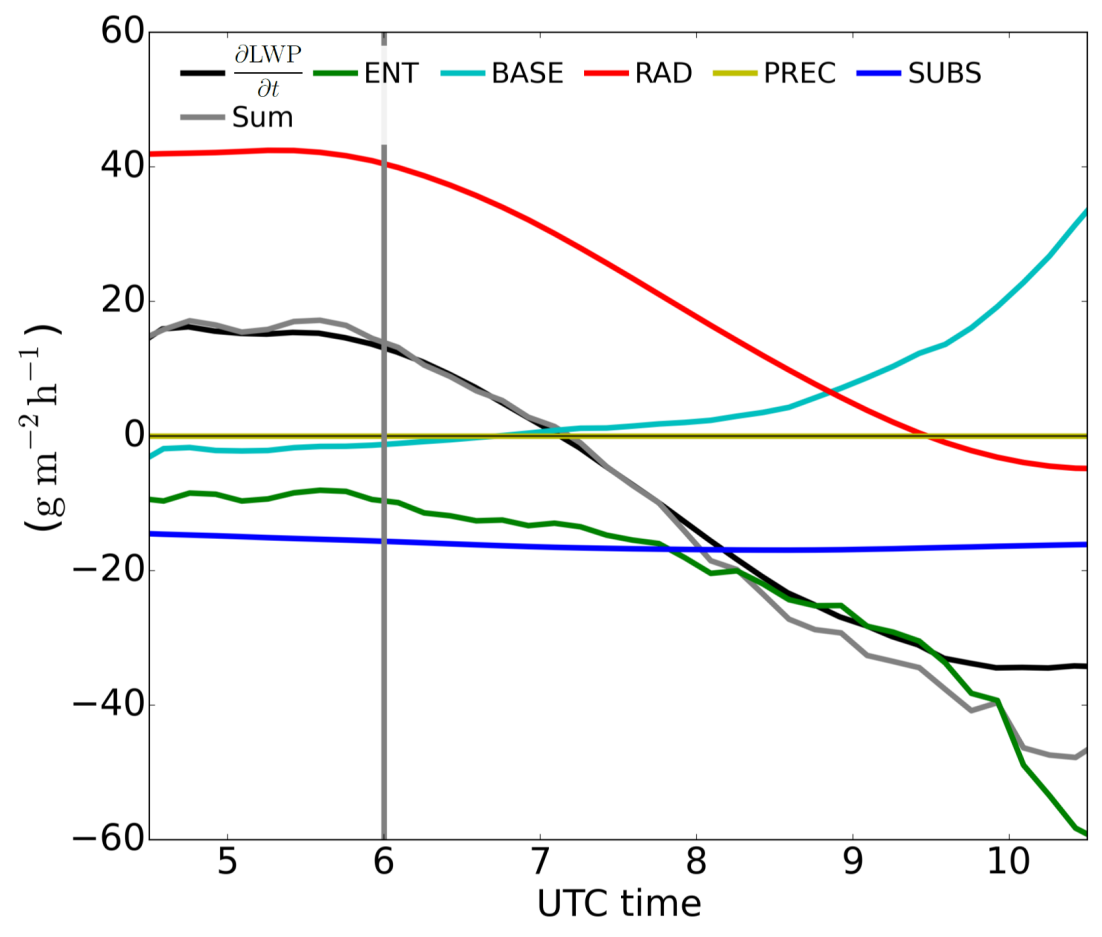

Figure 4.6: Time series of budget terms as defined in Eqs.4.2 and 4.3 with colors representing the terms as displayed in the legend, and the Sum grey line being the sum of all the terms on the RHS of Eq. 4.2. The vertical grey line indicates sunrise time.

this transition? To this end and relating to Fig. 4.2b, LWP is calculated and used as the metric to describe the state of the transition and calculate the budget derived by van der Dussen et al. (2014). The budget reads:

$$
\frac{\partial \mathrm{LWP}}{\partial t}=\mathrm{BASE}+\mathrm{ENT}+\mathrm{PREC}+\mathrm{RAD}+\mathrm{SUBS}
$$

with

$$
\begin{aligned}
\mathrm{BASE} & =\rho \eta\left(\overline{w^{\prime} q_{t}^{b}}-\Pi \gamma \overline{w^{\prime} \theta_{l}^{\prime}}\right) \\
\mathrm{ENT} & =\rho w_{e}\left(\eta \Delta q_{t}-\Pi \gamma \eta \Delta \theta_{l}-D \Gamma_{q_{l}}\right) \\
\mathrm{PREC} & =-\rho \delta P \\
\mathrm{RAD} & =\rho \eta \gamma \delta F_{r a d} \\
\mathrm{SUBS} & =-\rho D \Gamma_{q_{l}} w_{s}(h)
\end{aligned}
$$

with BASE representing the effect of turbulent fluxes at cloud base, ENT that of entrainment, PREC the effect of precipitation, RAD that of radiation, and SUBS the one due to subsidence. $\Delta q_{t}$ and $\Delta \theta_{l}$ are the jumps across the inversion layer for total water mixing ratio and liquid water potential temperature, respectively, defined as in van der Dussen et al. (2016): $\Delta \theta_{l}=\theta_{l}\left(z i^{+}\right)-\theta_{l}\left(z i^{-}\right)$and $\Delta q_{t}=q_{t}\left(z i^{+}\right)-q_{t}\left(z i^{-}\right) . \delta P$ and $\delta F_{\text {rad }}$ represent the difference in precipitation and net radiation, respectively, between the top of the inversion layer $z i^{+}$, assumed to be the same as Sc cloud top height in van der Dussen et al. 
(2016), and the Sc cloud base (van der Dussen et al., 2016). The rest of the variables in Eq. 4.3 are listed in Table 4.2 .

In short, this budget enables us to decompose the thinning or thickening of the cloud layer, quantified by a LWP tendency, and relate each contribution to the physical processes governing the stratocumulus clouds. To derive such budget van der Dussen et al. (2014) assumed the cloud layer to be horizontally homogeneous and vertically well mixed, implying a linear increase of the liquid water with height within the cloud layer following an adiabatic liquid water profile. The first hours of the simulation perfectly fit those conditions. However, after some hours the horizontal heterogeneities created in the Sc layer and the formation of convective clouds below (see Fig. 4.5) make these assumptions not to longer hold. Furthermore, the assumption of one well-mixed cloud layer breaks after 1000 UTC due to the warming by radiation and entrainment (Fig. 4.4). The distance between $z i^{+}$and ctop_Sc, assumed to be negligible by van der Dussen et al. (2014), increases with time up to $50 \mathrm{~m}$ at 1000 UTC. For this reason we focus our analysis on the first stage of the transition until 1000 UTC.

Before sunrise we observe in Fig 4.6 a net thickening of the cloud layer by almost 20 g $\mathrm{m}^{-2} \mathrm{~h}^{-1}$, i.e. a growth of about $15 \%$, driven solely by the longwave cooling at the cloud top (RAD term). During the entire experiment SUBS remains almost constant given the small variation of subsidence with height, showing a negative tendency of around $16 \mathrm{~g} \mathrm{~m}^{-2} \mathrm{~h}^{-1}$. The negative tendency by entrainment (ENT) is to a large extent initially due to the entrainment of warm air (second term in ENT in Eq. 4.3) since, as shown in Fig.4.1, the free tropospheric air has similar moisture content as the cloudy air. The thinning tendency of precipitation is small, accounting for up to $4 \mathrm{~g} \mathrm{~m}^{-2} \mathrm{~h}^{-1}$ when the cloud layer its thickest. The small contribution of PREC despite large LWP is explained by the microphysical characteristics of the region. The large CCN concentrations typical for SWA (300 $\mathrm{cm}^{-3}$ in our study) prevent any large effects of precipitation even in Sc with high liquid water content. Of similar magnitude is the effect by cloud base fluxes before sunrise: the turbulent transport of warm air (second term of BASE in Eq. 4.3) dominates over its moistening effect (first term of BASE in Eq. 4.3) at this time. Yet the negative net effect by BASE in LWP tendency is about ten orders of magnitude smaller than that of RAD.

After sunrise the warming effect of shortwave radiation increasingly offsets the longwave cooling at cloud top. This leads to a decreasing contribution of RAD to the thickening of the cloud layer. Due to this factor, the sign of LWP tendency changes at around 715 UTC. This is the time when the thinning leading to the eventual cloud breakup starts. Correlated to the shortwave radiation increase after sunrise, the surface-driven growth of the boundary layer leads to larger entrainment rates, thus increasing the warming of the cloud layer through the free-tropospheric engulfed air. An additional factor to the already mentioned warming explains the fast shift to more negative tendencies for the ENT term 
after 700 UTC: the increased drying through entrainment. This drying increases due to two factors enhancing $\Delta q_{t}$, from $-0.27 \mathrm{~g} \mathrm{~kg}^{-1}$ at $700 \mathrm{UTC}$ to $-1 \mathrm{~g} \mathrm{~kg}^{-1}$ at 1000 UTC: the moisture input in the boundary layer by the surface; and the growth of the boundary layer itself across a drier free troposphere. This larger moisture jump enhances the impact of entrainment by a) drying the cloud layer and b) enhancing the entrainment velocity as the difference in buoyancy between the cloud and free troposphere decreases. By the end of this period, at 1000 UTC, the positive contribution to LWP of cloud base fluxes (BASE) rises to up to $30 \mathrm{~g} \mathrm{~m}^{-2} \mathrm{~h}^{-1}$. This is explained by the increase of surface fluxes (Fig 4.2c) and surface buoyancy (Fig. 4.4e) as the available net radiation at surface grows. These changes lead to a larger contribution of the moistening ${\overline{w^{\prime} q_{t}^{\prime}}}^{b}$ term to BASE in Eq. 4.3, while the warming term including $\overline{w^{\prime} \theta_{l}^{\prime}}$ remains less variable for the first hours. Note that although the moisture flux increase at cloud base implies a growth of LWP in the budget, such growth may eventually lead to a dissipation of the cloud layer: increased surface moisture flux at surface and consequently, at cloud base, relates to enhanced buoyancy within the cloud layer, known to increase entrainment. Such accelerated entrainment leads to the warming of the upper cloud, and thus counteracts the mixing of the cloud layer necessary for the maintenance of the Sc.

Comparing the contributions in our case before sunrise to those of the first night in van der Dussen et al. (2016), we find a RAD term almost 30\% lower in our case. Given the similar LWP and $\theta_{l}$ jump above cloud top, we attribute the significant difference to the lack of a moisture jump here and thus, weaker cloud top radiative cooling. The BASE term reached values of about $60 \mathrm{~g} \mathrm{~m}^{-2} \mathrm{~h}^{-1}$ in van der Dussen et al. (2016), while we found very little contribution of such term during the morning due to the compensation of moistening and warming effect of turbulent fluxes. This large difference compared to a marine case shows the relevance of the land surface, as the moistening is limited here and counteracted by a larger warming through turbulent fluxes at cloud base compared to a marine case. The nighttime ENT term is in our case about two to three times smaller than in van der Dussen et al. (2016), explained by larger turbulence created by a stronger RAD in their study. All in all, the total tendency $\frac{d \mathrm{LWP}}{d t}$ is in the same order of magnitude for both cases although the drivers remain quite different. The increasing negative contribution to the LWP budget by entrainment at daytime is consistent with the Sc over land case by Ghonima et al. (2016). We find our case to fall between their cases with fixed Bowen ratios of $B o=0.1$ and $B o=1$, as we observe a nearly $B o=0$ during night growing up to 0.6 during the day in the current case, similar to the measured conditions (Fig. $4.2 \mathrm{c}$ ). This indicates the advantage of having a land surface model correctly partitioning the available net energy into surface and latent heat fluxes. The BASE term behaves in our case similar to their $B o=0.1$ case as it also shows a positive contribution to cloud thickening or LWP increase. 


\subsubsection{Effect of wind and wind shear in the transition}

\subsubsection{Nighttime effects}

We showed that the transition from stratocumulus to cumulus over land for typical SWA conditions can take place under windless conditions. Given the recurrent presence of wind and low level jet in the morning during the observational campaign, it is interesting to further investigate the effects that wind has on the transition. Thus, we extend the previous results considering the further effects that mean wind (MEAN) and additional wind shear at cloud top (SHEAR) have on the transition described. We include in Table 4.1 the timing and magnitude of the reference metrics for each experiment. Under cloudless conditions, the effect of shear at surface as well as at boundary layer top acts as a local source of Turbulence Kinetic Energy (TKE) (Conzemius and Fedorovich, 2006). In our case, such modifications in turbulence may affect the evolution of the cloud transition described in previous sections. First, we show in Fig. 4.7 the relative differences between the terms defined in Eq. 4.3 as part of the LWP budget. Following van der Dussen et al. (2016), we show the accumulated difference, starting at 430 UTC, on the LWP tendency due to each term between MEAN or SHEAR and the reference simulation REF. Taking the precipitation contribution PREC as an example, we calculate:

$$
\Delta \mathrm{LWP}^{\mathrm{PREC}}(t)=\int_{4 \text { 30UTC }}^{t} \operatorname{PREC}\left(t^{\prime}\right)-\operatorname{PREC}^{R E F}(t)^{\prime} d t^{\prime}
$$

and similarly for all the other terms present in Eq. 4.2.

The presence of a light mean wind $\left(3 \mathrm{~m} \mathrm{~s}^{-1}\right)$ on the entire domain has only minor effects on the first part of the transition: Figure 4.7 shows a slightly larger LWP for the MEAN experiment compared to REF. The larger LWP is driven by the increased contribution of the turbulent fluxes at cloud base (BASE). For both MEAN and SHEAR it shows a thickening contribution already before sunrise, whereas it was a net thinning contribution in REF experiment. The change in BASE is explained as follows: wind enhances latent heat flux as well as turbulent generation near surface, favoring the transport of moisture to the cloud layer. The enhanced turbulence generation near surface due to the wind, both in MEAN and SHEAR, is visible in the lower part of Fig. 4.8a. We show there the contributions by the buoyancy and shear terms, $B$ and $S$ respectively, to the TKE tendency budget and the good agreement on surface TKE between our experiments and the observations. Enhanced LWP in nighttime Sc by the presence of wind was also found by Kazil et al. (2016) and attributed to enhanced buoyancy production of TKE due to latent heat release in cloud updrafts. Such findings coincide with the enhanced buoyancy term $B$ for MEAN in Fig. 4.8a. Precipitation, acting as a negative feedback on LWP, attenuates the effect by BASE in the total tendency of LWP. The remaining terms show little variation between REF and MEAN. 

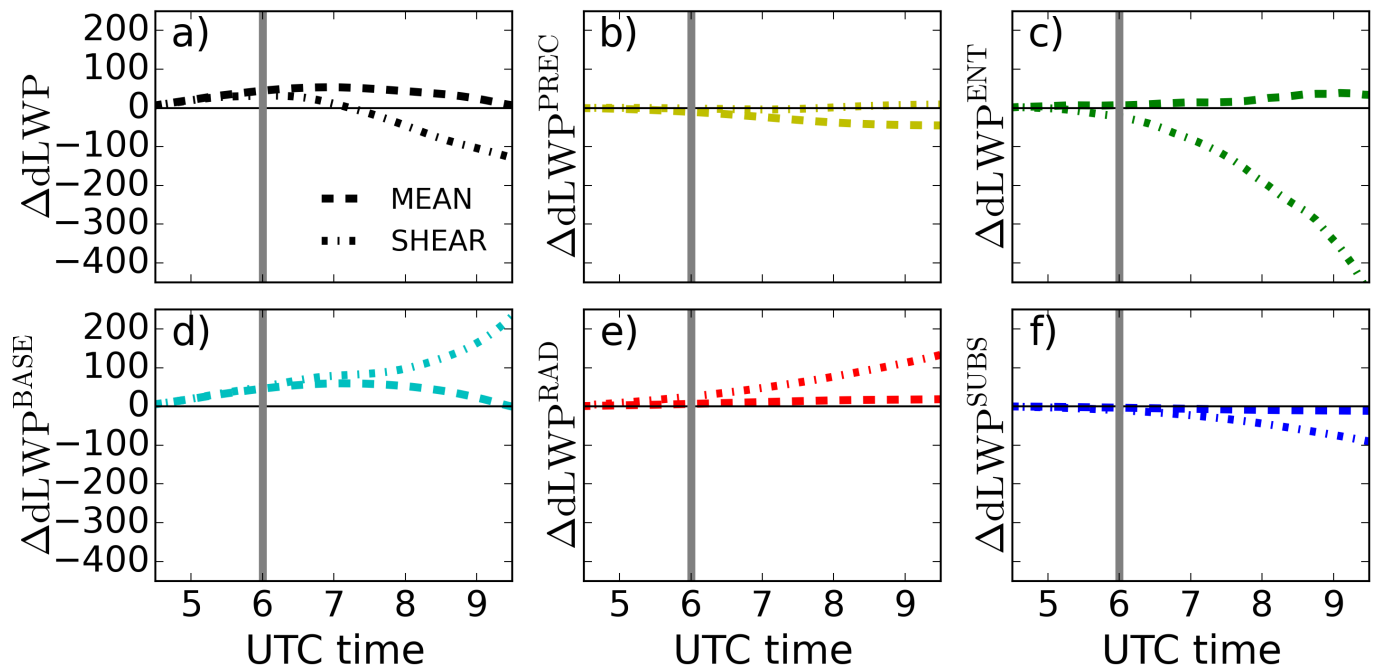

Figure 4.7: Time series of accumulated differences between MEAN and REF (dashed) and between SHEAR and REF(dotted-dashed) for each term defined in Eq. 4.2 and calculated following Eq.4.4.

Wind shear at the top of the cloud layer introduces larger changes: it is known to enhance TKE locally but with a total negative effect on cloud TKE due to reduced buoyancy production (Wang et al., 2012) and to enhance entrainment at cloud top (Mellado, 2017). Before sunrise, cloud layer LWP as well as cloud base and cloud top heights (Fig. 4.9d) show small differences between SHEAR and MEAN experiments. SHEAR shows systematic lower LWP (not shown) but a thicker Sc cloud layer, e.g. $\simeq 40 \mathrm{~m}$ thicker before sunrise, due to increased entrainment velocities. Similarly, we also find a turbulent and clear sublayer between the cloud top and the inversion layer top in SHEAR (Fig. 4.9a). These results agree with the findings by Wang et al. (2008) and McMichael et al. (2019), who studied cloud-top shear effects on marine Sc clouds. Such agreement reinforces the analogy between the night-time Sc cloud studied here before sunrise and the typical marine Sc, given the low values of the surface fluxes.

Although with similar tendencies in the LWP budget before sunrise, the sources for turbulence and, thus, mixing within the cloudy layer are different in MEAN and REF compared to SHEAR. As shown in Fig. 4.8a, SHEAR shows a much larger contribution by wind shear $S$ to the TKE tendency at cloud top, up to $1.5 \mathrm{~m}^{2} \mathrm{~s}^{-3}$ or more than five times the local buoyancy contribution $B$ within the cloud layer. SHEAR also exhibits a slightly lower contribution by buoyancy from cloud top to surface. The larger contribution by $S$ is a consequence of the varying wind speed in the cloud boundary, while the cause for lower $B$ throughout the whole layer lies in the weaker cooling at cloud top (not shown) due to the shear-induced broader inversion layer (Mellado, 2017): the inversion layer is more than $80 \mathrm{~m}$ thick before sunrise at SHEAR, while is about $40 \mathrm{~m}$ in REF and MEAN. The increase in the depth of this layer results in a decrease of the longwave cooling at cloud 

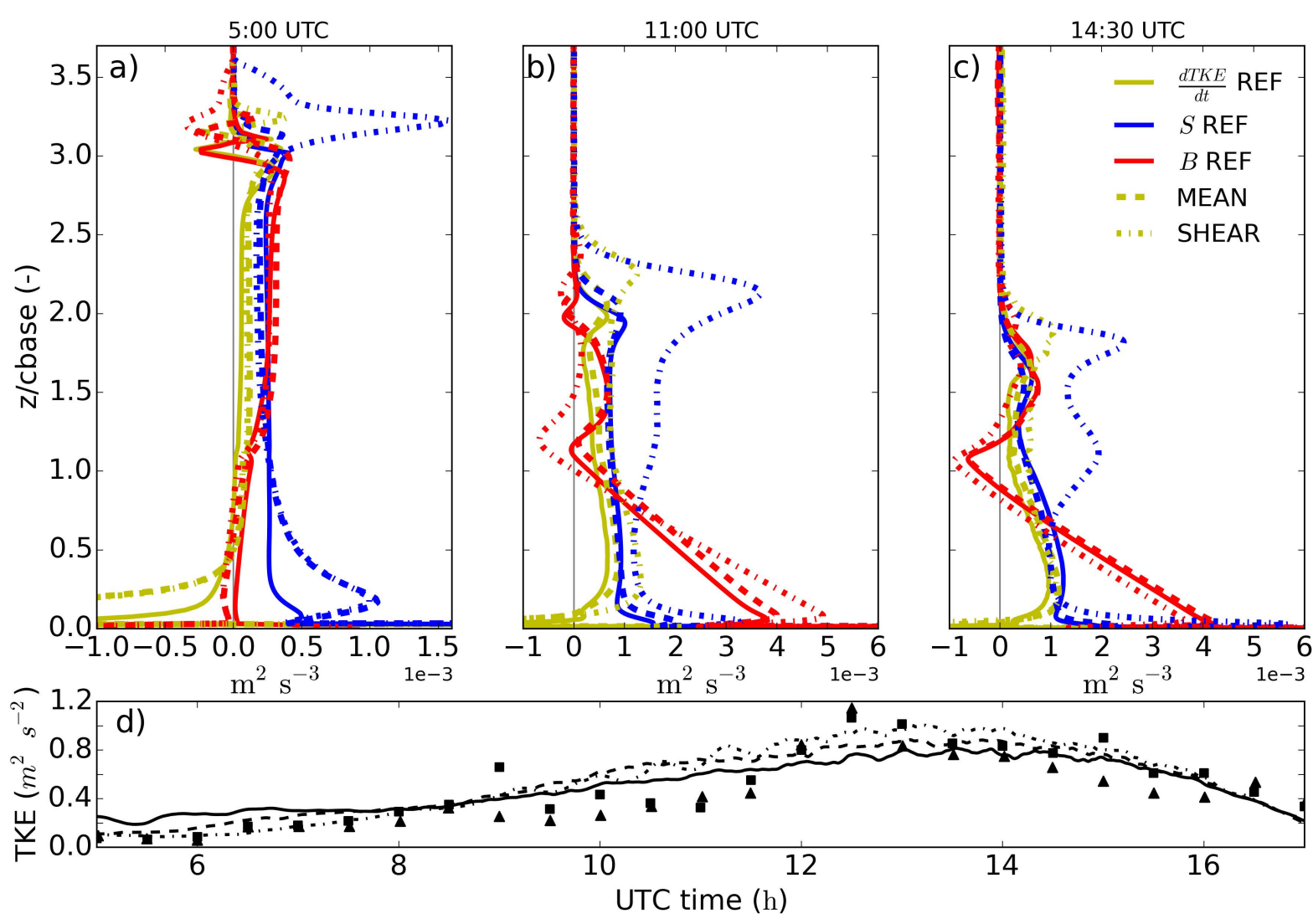

Figure 4.8: Slab averaged vertical profiles of 20-min averaged turbulent kinetic energy tendency (yellow), shear contribution (blue) and buoyancy contribution(red) for REF (full), MEAN (dashed) and SHEAR (dotted-dashed) at 500 (a), 1100 (b) and 1430 UTC(c). The height is normalized by cloud base height at the vertical axis. In (d) and following the same line settings, time series of the simulated turbulent kinetic energy at $10 \mathrm{~m}$ high and, in triangles and squares, as observed by two independent stations at the Savé supersite on $26^{\text {th }}$ June 2016.

top, from about $-6.1 \mathrm{~K} \mathrm{~h}^{-1}$ in MEAN or REF to $-4 \mathrm{~K} \mathrm{~h}^{-1}$ in SHEAR, as the gradients are smoothened and the time air is exposed to the cooling is decreased (Yamaguchi and Randall, 2008). Wang et al. (2008, 2012) also found weaker cooling at cloud top and a thicker inversion layer on sheared Sc.

Our findings thus suggest that while mean wind during the night has no major effects, nighttime cloud top shear hampers the cloud growth by reducing the cooling allowed at the cloud top. The little differences between MEAN and REF reinforce the idea that the turbulence generated by wind shear at surface in MEAN needs to be transported up to the top of the well-mixed layer to affect entrainment and the overall dynamics of the boundary layer. Yet the traveling turbulence is subject along its rise from surface to cloud top to the turbulent cascade, partly dissipating and having a reduced impact on the entrainment zone (Conzemius and Fedorovich, 2006). On the contrary, local shear at 

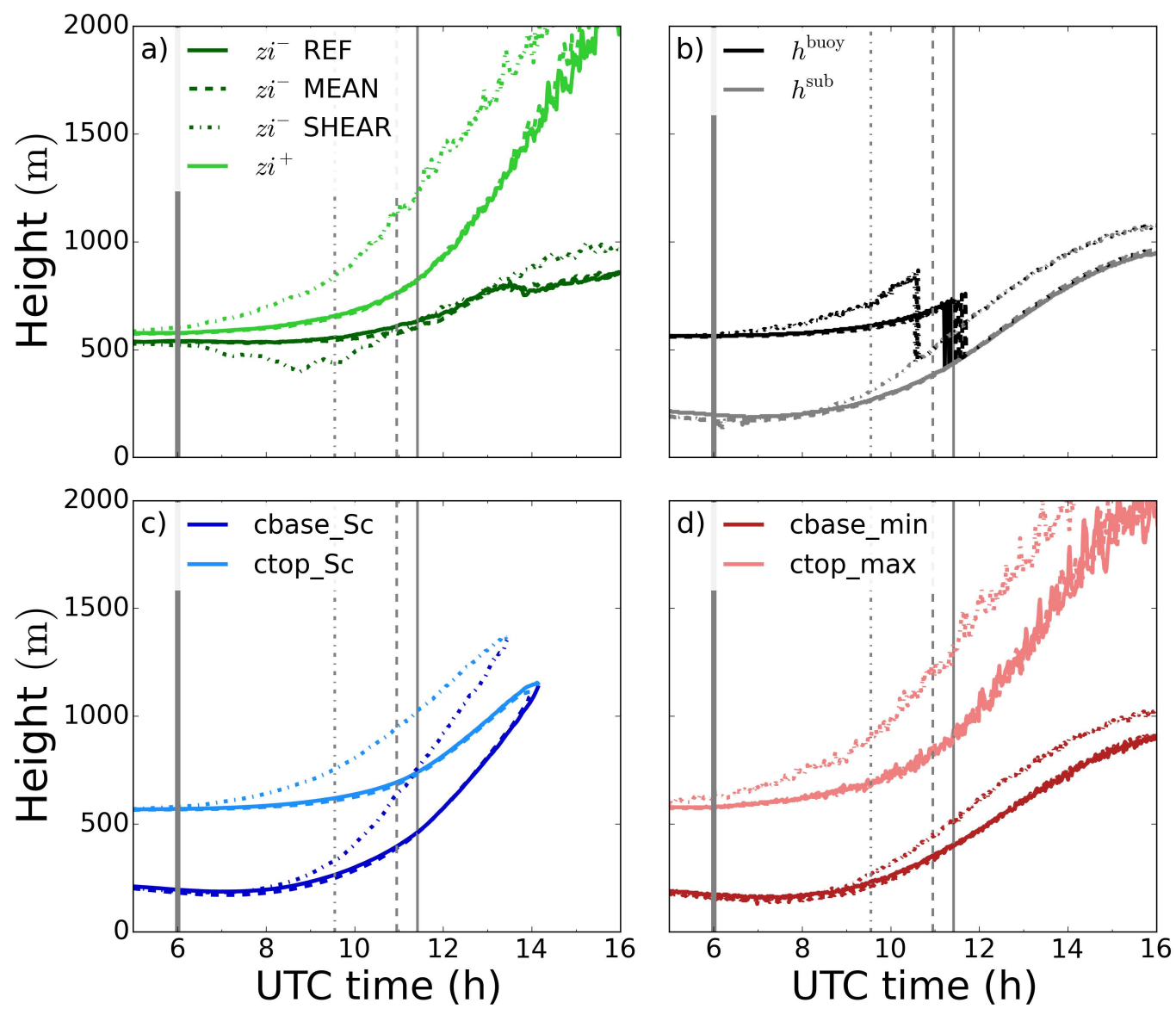

Figure 4.9: As in Fig. 4.5 for REF (full), MEAN (dashed) and SHEAR(dotted-dashed).

cloud top locally generates turbulence to immediately affect entrainment and boundary layer growth. Thus we find SHEAR to have larger effects in the Sc-Cu transition. We show in the coming section that the presence of shear at cloud top after sunrise promotes a faster breakup of the cloud layer.

\subsubsection{Daytime effects}

After sunrise the shear effects drive the cloud layer towards lower LWP due to enhanced entrainment of warm air (Fig. 4.7c). The enhanced entrainment velocity in SHEAR is more visible ( $h$ growth as proxy for $w_{e}$ in Fig. $4.9 \mathrm{~b}$ ) after the decoupling of the cloud layer and surface between 900 and 1000 UTC (see Table 4.1). We attribute the increase in $w_{e}$ not only to the presence of local shear at cloud top, but also to the positive feedback between surface fluxes and cloud thinning (Ghonima et al., 2016), further reinforced by wind shear in this case: the slightly lower LWP after sunrise in SHEAR enhances the turbulent fluxes both at surface (Fig. 4.8b,c) and cloud base (Fig. 4.7d). Larger daytime turbulence within the cloud layer leads locally to thinning of the inversion layer, allowing 
for a locally enhanced wind shear (Mellado, 2017) and, thus, further entrainment which will lead to a more negative rate for $\frac{d \mathrm{LWP}}{d t}$ and the subsequent increase of surface fluxes. Furthermore, the accelerated growth of the boundary layer in SHEAR leads to a larger moisture difference between the cloud layer and the air above, thus further reinforcing the negative effects of entrainment through additional drying in ENT (Fig. 4.7) in the tendency of cloud layer LWP.

On the other hand, radiative cooling (RAD) remains a positive contribution for $\frac{d \mathrm{LWP}}{d t}$ for longer time (see Fig. $4.7 \mathrm{~d}$ ). The reason is the thicker integration layer, caused by wind shear, over which RAD is evaluated. This layer ranges from cbase_Sc to inversion layer top $\left(z i^{+}\right)$for the budget in Eqs. 4.2,4.3. As assumed by van der Dussen et al. (2016), $z i^{+}$ and ctop_Sc agree quite well for thin inversion layers such as the one during night without shear in REF (see Fig. 4.5) and the choice is unimportant. The agreement worsens when shear is present, as the inversion layer thickens and ctop_Sc and $z i^{+}$show larger discrepancies (Fig. 4.9), as also shown by Wang et al. (2012). This thicker layer over which RAD is calculated explains the larger divergence in the net radiative flux between the cloud base and $z i^{-}$. Thus, a sensitive point for the discussion is the definition of the limits: one may wonder if the larger contribution to LWP gain of RAD in SHEAR may be an artifact of the boundaries selected for the budget in Eq.4.2. Using other limits at the top, such as ctop_Sc or cloud top, lead however to a worse closure of the budget.

The negative LWP tendency is hampered in SHEAR by the positive contribution of BASE (Fig. 4.7d). The increase in BASE is explained as part of the positive feedback stated above: given the lower LWP at sunrise more shortwave radiation reaches the surface, increasing the surface fluxes, specially, the latent heat flux. Thus we deduce that the initial lower LWP in SHEAR accelerates the further thinning and eventual breakup of the cloud layer due to two factors enhancing entrainment: the direct enhancement due to local shear at cloud top, and the indirect one due to larger surface fluxes and boundary layer growth. This is represented in the LWP budget by more negative and positive values for ENT and BASE, respectively.

Figure 4.9 completes the analysis of wind sensitivity after the decoupling of the cloud layer. In agreement with the previous explanation, MEAN evolves similarly to REF as the wind-driven increase in surface fluxes is negligible compared to the boundary layer dynamics. Larger entrainment velocities accelerate the growth of the boundary layer in

Table 4.1: Values and time of the main features in the stratocumulus to cumulus transition for the three experiments REF, MEAN and SHEAR.

\begin{tabular}{|c|c|c|c|c|c|c|c|}
\hline Experiment & $\begin{array}{c}\text { Max LWP } \\
\left(\mathrm{g} \mathrm{m}^{-2}\right)\end{array}$ & $\begin{array}{c}\text { Start of } \\
\text { convective phase }\end{array}$ & $\frac{d \text { LWP }}{d t}<0$ time & $\begin{array}{c}\text { Decoupling time } \\
\left(\overline{w^{\prime} \theta_{v}^{\prime}} \text { sub }<0\right)\end{array}$ & $\begin{array}{c}h=h^{\mathrm{sub}} \\
\text { time }\end{array}$ & $\begin{array}{l}\text { Breakup time } \\
\quad(c c<1)\end{array}$ & $\begin{array}{c}\text { CBL time } \\
\left(r_{\theta_{v}}<-0.15\right)\end{array}$ \\
\hline REF & 174.4 & 0655 & 0707 & 1105 & 1108 & 1125 & 1302 \\
\hline MEAN & 185.6 & 0651 & 0706 & 1114 & 1123 & 1058 & 1209 \\
\hline SHEAR & 173.9 & 0653 & 0644 & 0919 & 1028 & 0933 & 1043 \\
\hline
\end{tabular}



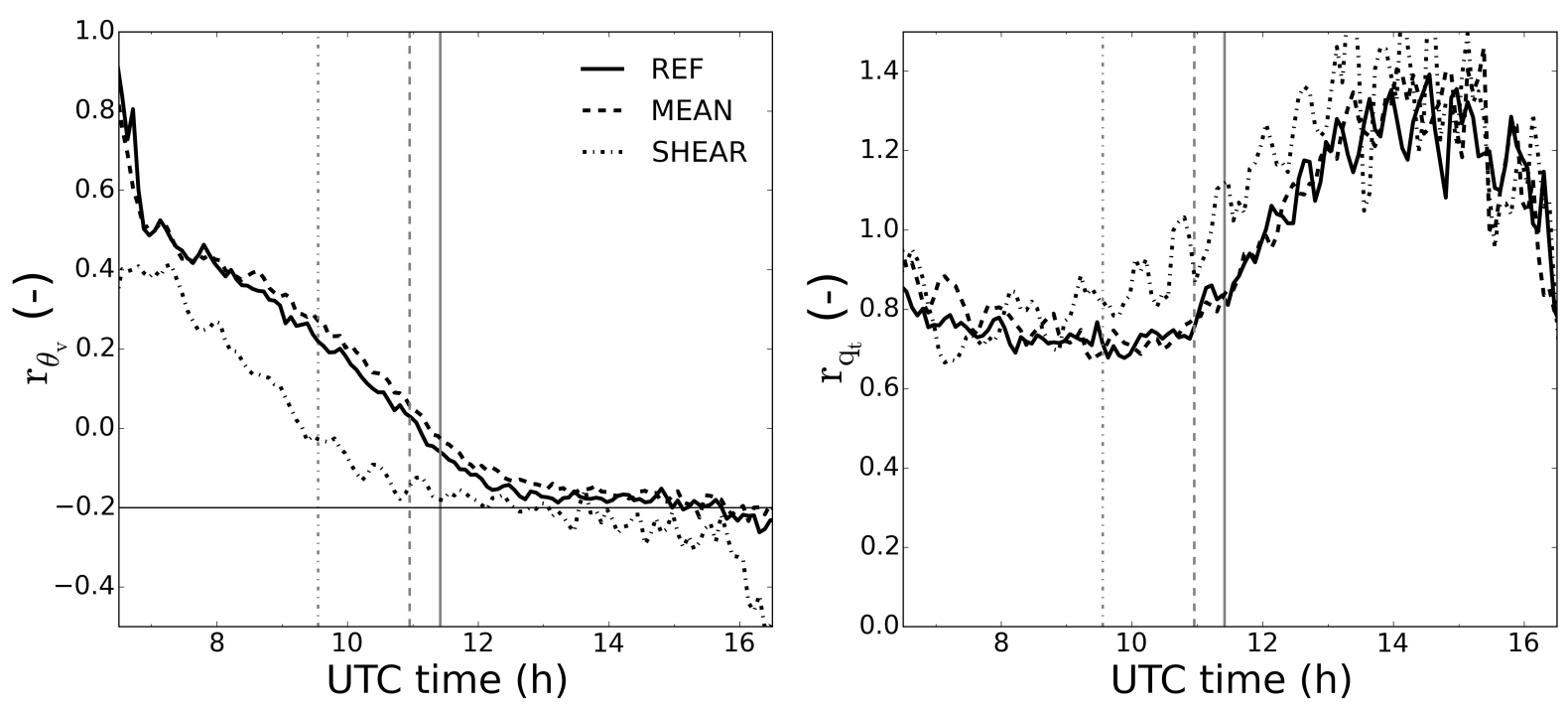

Figure 4.10: Time series of subcloud layer top to surface buoyancy flux (left) and total moisture flux (right) ratio for REF (full lines), MEAN (dashed) and SHEAR (dotted-dashed). Vertical lines represent cloud breakup for each experiment.

SHEAR, as well as the rise of cloud top and cloud base of both the total and Sc cloud layers (Figs. 4.9c,d). The faster growth of the boundary layer with shear at its top is a well documented feature (Conzemius and Fedorovich, 2006; Liu et al., 2018). The fasterrising cloud layer in SHEAR coincides with an earlier negative buoyancy flux minimum at cloud base and, thus, an earlier decoupling of the cloud layer by almost 2 hours (Table 4.1). Besides the shear effects the larger surface fluxes due to a lower LWP after sunrise also explain the faster growth of the subcloud layer buoyancy flux minimum. Similarly, the inversion layer grows faster in SHEAR due to both a decreasing $z i^{-}$and a growing $z i^{+}$. Following the accelerated processes in SHEAR, the breakup of the cloud layer takes place about 2 hours earlier than in REF.

\subsubsection{Representation by large scale metrics}

A transition from stratocumulus to shallow cumulus represented as a continuum and over a period spanning several hours, such as the one shown here, poses challenges to coarser resolution models in correctly representing cloud fraction, inversion layer height or thickness as well as buoyancy source(s). To quantify the transition and explain its possible drivers beyond 1000 UTC we calculate two metrics traditionally used in larger scale models: the ratio between subcloud layer top buoyancy flux, i.e. the buoyancy flux evaluated at $h^{\text {sub }}$, and surface buoyancy flux $r_{\theta_{v}}=\frac{\overline{w^{\prime} \theta_{v}^{\prime}}}{{\overline{w^{\prime} \theta_{v}^{\prime}}}^{s u b}}$ and its analogous for the total moisture flux $r_{q_{t}}=\frac{\overline{w^{\prime} q_{t}^{s u b}}}{\overline{w^{\prime} q_{t}^{\prime}}}$ in Fig. 4.10. $r_{\theta_{v}}$ is frequently used to parameterize entrainment velocities at boundary layer top, while $r_{q_{t}}$ provides information on the transport of moisture 
from surface to cloud and subcloud layer. These two metrics show the impact of the surface fluxes, in terms of buoyancy and moisture, on the boundary layer and capture the dynamics of it.

$r_{\theta_{v}}$ shows a linearly decreasing trend, showing the lowering transport of buoyancy to the cloud layer as the cloud-top driven circulation weakens, the surface buoyancy flux grows and so does the slope of the linearly decreasing vertical buoyancy flux. Around 1100 UTC the sign of $r_{\theta_{v}}$ reverses (see also nearly negative buoyancy flux minimum at $h^{\text {sub }}$ in Fig. 4.4d). This explicitly indicates the decoupling between the cloud layer and the surface. After 1200 UTC $r_{\theta_{v}}$ approaches the typical ratio of -0.2 for dry CBLs (Stull, 1988) until the decay of turbulence generation at surface by the end of the experiment. Similarly, $r_{q_{t}}$ presents slightly decreasing values from 0.8 to 0.7 after sunrise. This was also found in other studies of marine Sc by de Roode et al. (2016), mentioning that $r_{q_{t}}<1$ implies a net moistening of the subcloud layer. As shown in Fig. 4.6, the moistening and warming of the cloud layer by turbulent fluxes from the surface almost offsets each other in terms of LWP impact on for the first hours after sunrise. After the shift in $h$ and before the breakup at 1130 UTC we observe growing values for $r_{q_{t}}$ related to increasing latent heat flux at surface. After $1200 \mathrm{UTC}$ we find values higher than 1, indicating a net drying of the subcloud layer and consequent moistening of the cloud layer by surface evapotranspiration. MEAN shows little variation from REF, reinforcing the small effect of the mean wind in the transition. The only remarkable difference is a one hour delay in reaching values of $r_{\theta_{v}}$ near typical CBL of -0.2 , due to the fact under convective conditions that mean wind may hamper the turbulent updrafts from the surface to the boundary layer top, thus reducing the related entrainment (Liu et al., 2018). SHEAR shows a qualitatively similar pattern to REF after sunrise with an earlier shift on the sign of $r_{\theta_{v}}$ of about two hours. Afterwards, $r_{\theta_{v}}$ reaches values lower than -0.2 in SHEAR. This suggests that as found by Conzemius and Fedorovich (2006), the buoyancy entrainment flux is enhanced compared to clear CBLs.

\subsection{Conclusions}

Based on observations of the DACCIWA project in southern West Africa we designed a numerical experiment to reproduce the transition from nighttime stratocumulus to daytime cumulus clouds over land. Special emphasis is placed on the the stratocumulus deck breakup and the role of the surface and boundary-layer processes. This was done by means of a Large Eddy Simulation with an interactive radiation scheme and a plant-mechanistic land surface model, allowing for coupled responses of radiative profiles and surface fluxes to changes in the thermodynamic fields and surface conditions. Numerical experiments were evaluated against a complete set of observations.

We quantified the transition in terms of inversion layer height and thickness, cloud-top 
and cloud-base heights and boundary layer height. These metrics remain largely constant over time during the night and similar to typical marine stratocumulus clouds, and start diverting from these values a few hours after sunrise. The main drivers are the increased entrainment due the enhanced turbulence driven by the surface fluxes and, to a lesser extent, the shortwave radiative warming at cloud top. We further showed how temperature, vertical velocity distributions and buoyancy and radiative fluxes vary during the transition period. Notable features during the transitions are the decoupling of the cloud layer by 11 UTC supported by 1) two independent well mixed layers seen in the temperature profiles and 2) a negative subcloud buoyancy flux minimum. The radiative fluxes shift from exerting a net cooling effect to a warming within the cloud layer which, in addition to the warming by entrainment, leads to the mentioned decoupling.

We further described and quantified the varied physical processes that maintain and thin the stratocumulus cloud layer using the LWP budget (van der Dussen et al., 2016). The radiative term is the most dominant process contributing to LWP increase during nighttime, while its contribution decreases after sunrise and becomes a sink of LWP due to increasing shortwave radiation warming. Subsidence has a negative and fairly constant contribution to the budget during the whole transition. Precipitation and cloud base fluxes, the latter driven by the cloud top cooling circulation, have almost no effects during the night. As the day progresses, the moisture flux from the surface contributes increasingly to the growth of LWP. Entrainment has a negative and nearly constant contribution during night. After sunrise, the entrainment induced LWP thinning intensifies due to cloud layer rise and the increasing moisture difference between the cloud layer and the air above.

Lastly we investigated the effect of wind on the transition: two additional experiments were performed along with the windless reference experiment: one experiment with a mean wind of $3 \mathrm{~m} \mathrm{~s}^{-1}$ at all heights and another with an additional wind jump at cloud-top of $5 \mathrm{~m} \mathrm{~s}^{-1}$ and further increase of $5 \mathrm{~m} \mathrm{~s}^{-1} \mathrm{~km}^{-1}$ above. The geostrophic wind was assumed to be identical to the prescribed wind in each experiment. The aim was to represent the main features of a recurrent low level jet observed in the region during the nighttime and morning. We found the mean wind to have almost no impact on the transition. However, the shear at cloud top had larger effects. Before sunrise, the inversion layer was thicker and the TKE generation by shear higher at cloud top at the expense of lower generation by buoyancy. These features are typical of sheared marine Sc. After sunrise, shear accelerated cloud thinning, boundary layer growth and the transition to a convective boundary layer. This was due to the direct effect of shear on entrainment growth similar to clear convective boundary layers, but also to the enhanced surface fluxes as cloud layer thinned faster. The related enhanced entrainment contributed to a faster thinning of the cloud layer, leading to a breakup two hours earlier than the no-wind experiment.

We calculated widely-used relationships that characterize the prototypical clear and 
cloudy boundary layer to determine their ability in reproducing the transition. We find that the ratio between the subcloud layer entrainment and the surface turbulent buoyancy fluxes $r_{\theta_{v}}$ decreases linearly with time during the transition, starting from initial values of $r_{\theta_{v}}=1$ and reaching typical dry convective values of -0.2 about one hour after the Sc deck break up at about 1230 UTC. The analogous moisture ratio shows a slight decrease from 0.8 to 0.7 until the shift in buoyancy flux minimum. After the shift, $r_{q_{t}}$ increases reaching values above 1 , thus moistening the cloud layer. Mean wind leaves the transition representation by $r_{\theta_{v}}$ and $r_{q_{t}}$ unaffected, except for a 1 hour delay in reaching CBL values for $r_{\theta_{v}}$. In contrast, the presence of cloud top shear accelerates by 2 hours the evolution of both $r_{\theta_{v}}$ and $r_{q_{t}}$. Furthermore, $r_{\theta_{v}}$ reaches values more negative than -0.2 after breakup. These findings reveal the relevance of the land-atmosphere feedbacks on the stratocumulus thinning and cloud transition, and the impact of wind on it. 


\section{Acknowledgements}

The authors would like to thank the work of all people involved in the measurement campaign of DACCIWA, and in particular to all the people who contributed to the Save supersite. The first author acknowledges Maurin Zouzua for providing the cloud cover observations, Anna-Lena Deppenmeier for providing ERA-interim data and Shantonu Abe Chatterjee for the useful tips during the writing process. The research leading to these results has received funding from the European Union 7th Framework Programme (FP7/2007-2013) under Grant Agreement no. 603502 (EU project DACCIWA: Dynamicsaerosol-chemistry-cloud interactions in West Africa). The numerical simulations were performed with the supercomputer facilities at SURFsara and financially sponsored by the Netherlands Organisation for Scientific Research (NWO) Physical Science Division (project number SH-312-15). This study was supported by the grant from the NWOALW Open Programme (824.15.013).

The data obtained during the DACCIWA campaign, including the Savè supersite, is freely available on the SEDOO database (http://baobab.sedoo.fr/DACCIWA/) (Derrien et al., 2016; Handwerker et al., 2016; Kohler et al., 2016). The DALES code is freely available for download at https://github.com/dalesteam/dales. 


\section{A List of symbols in Chapter 4}

Table 4.2: List of symbols in Chapter 4

\begin{tabular}{|c|c|c|}
\hline Variable & Name & Units \\
\hline$B$ & Buoyancy term in TKE tendency equation & $\mathrm{m}^{2} \mathrm{~s}^{-3}$ \\
\hline Bo & Bowen ratio & $(-)$ \\
\hline$c c$ & Cloud cover & $(-)$ \\
\hline$c_{p}$ & dry air specific heat & $J \mathrm{~kg}_{\mathrm{a}} \mathrm{K}^{-1}$ \\
\hline cbase_Sc & Stratocumulus cloud base height & $\mathrm{m}$ \\
\hline ctop_Sc & Stratocumulus cloud top height & $\mathrm{m}$ \\
\hline$D$ & cloud layer depth & $\mathrm{m}$ \\
\hline$F$ & Net radiative flux & $\mathrm{W} \mathrm{m}^{-2}$ \\
\hline$F_{0}$ & Net radiative flux at surface & $\mathrm{W} \mathrm{m}^{-2}$ \\
\hline$g$ & gravitational acceleration & $\mathrm{m} \mathrm{s}^{-2}$ \\
\hline$h$ & Boundary layer height & $\mathrm{m}$ \\
\hline$h^{\mathrm{sub}}$ & Subcloud layer height & $\mathrm{m}$ \\
\hline$L E$ & Latent heat flux & $\mathrm{W} \mathrm{m}^{-2}$ \\
\hline LWP & Liquid Water Path & $\mathrm{g}_{\mathrm{w}} \mathrm{m}^{-2}$ \\
\hline$q_{s}$ & Saturation specific humidity & $\mathrm{g}_{\mathrm{w}} \mathrm{Kg}_{\mathrm{a}}^{-1}$ \\
\hline$q_{t}$ & Total specific humidity & $g_{w} K_{a}^{-1}$ \\
\hline$R d$ & dry air gas constant & $\mathrm{J} \mathrm{kg}_{\mathrm{a}} \mathrm{K}^{-1}$ \\
\hline$r_{\phi}$ & Subcloud to surface $\overline{w^{\prime} \phi^{\prime}}$ ratio & $(-)$ \\
\hline$S$ & Shear term in TKE tendency equation & $\mathrm{m}^{2} \mathrm{~s}^{-3}$ \\
\hline$S H$ & Sensible heat flux & $\mathrm{W} \mathrm{m}^{-2}$ \\
\hline$S_{w}$ & Skewness of vertical velocity $w$ & $(-)$ \\
\hline$T$ & Temperature & $K$ \\
\hline $\mathrm{U}$ & Horizontal windspeed & $\mathrm{m} \mathrm{s}^{-1}$ \\
\hline$w_{e}$ & Entrainment velocity & $\mathrm{m} \mathrm{s}^{-1}$ \\
\hline$w_{\text {subs }}$ & Subsidence & $\mathrm{m} \mathrm{s} \mathrm{s}^{-1}$ \\
\hline$\overline{w^{\prime} \phi^{\prime}}$ & Turbulent flux of $\Phi$ & $\mathrm{m} \mathrm{s}^{-1}[\Phi]$ \\
\hline$\overline{w^{\prime} \phi^{\prime}}$ & Turbulent flux of $\Phi$ at cloud base & $\mathrm{m} \mathrm{s}^{-1}[\Phi]$ \\
\hline$z i^{+}$ & Inversion layer top height & $\mathrm{m}$ \\
\hline$z i^{-}$ & Inversion layer bottom height & \\
\hline$\gamma$ & & $\mathrm{g}_{\mathrm{w}} \mathrm{Kg}_{\mathrm{a}}^{-1} \mathrm{~K}^{-1}$ \\
\hline$\Gamma_{q_{l}}$ & $g \eta\left(\frac{q_{s}}{R_{d} T}-\frac{\gamma}{c_{p}}\right)$ & $\mathrm{g}_{\mathrm{w}}$ \\
\hline$\delta F_{r a d}$ & $\begin{array}{l}\text { Difference in net radiation } \\
\text { between } z i^{+} \text {and cbase_Scu }\end{array}$ & $\mathrm{W} \mathrm{m}^{-2}$ \\
\hline$\delta P$ & $\begin{array}{l}\text { Difference in precipitation } \\
\text { between } z i^{+} \text {and cbase_Scu }\end{array}$ & $\mathrm{g}_{\mathrm{w}} \mathrm{g}_{\mathrm{a}}^{-1} \mathrm{~m} \mathrm{~s}^{-1}$ \\
\hline$\Delta q_{t}$ & $q_{t}$ jump along inversion layer & $\mathrm{g}_{\mathrm{w}} \mathrm{Kg}_{\mathrm{a}}^{-1}$ \\
\hline$\Delta \theta_{l}$ & $\theta_{l}$ jump along inversion layer & $\mathrm{K}$ \\
\hline$\eta$ & $\begin{array}{l}\text { Thermodynamic factor } \\
\text { (see van der Dussen et al. (2014)) }\end{array}$ & $(-)$ \\
\hline$\theta$ & Potential temperature & $\mathrm{K}$ \\
\hline$\theta_{l}$ & Liquid water potential temperature & $\mathrm{K}$ \\
\hline$\theta_{v}$ & Virtual potential temperature & $\mathrm{K}$ \\
\hline$\Pi$ & Exner function & $(-)$ \\
\hline$\rho$ & Air density & $\mathrm{Kg}_{\mathrm{a}} \mathrm{m}^{-3}$ \\
\hline
\end{tabular}





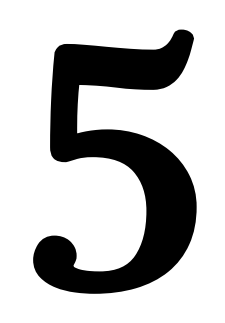

\section{Emergent features on cloud convection by coupling $3 \mathrm{D}$ radiation and surface on Large Eddy Simulations}

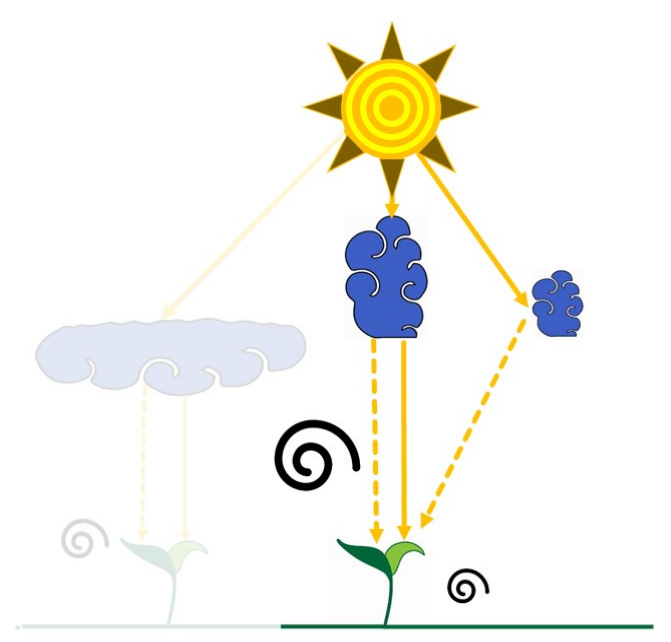

This chapter is in preparation to be submitted as:

Pedruzo-Bagazgoitia, X., Veerman, M., Jakub, F., van Heerwaarden, C. C., and Vilà-Guerau de Arellano, J. (In preparation). Emergent features on cloud convection by coupling $3 \mathrm{~d}$ radiation and surface on large eddy simulation 


\section{Abstract}

Current atmospheric models usually assume a one-dimensional direction for the radiation transfer, only allowing for upwards or downwards components of light. This is a relevant limitation, as horizontal traveling of radiation is constrained and observed features such as cloud-shade tilting are neglected. The present study analyzes the radiative and dynamical effects of using an innovative and relatively fast three-dimensional radiative scheme in the diurnal cycle of a shallow cumulus case over the Amazonian rainforest. To investigate the distinct effects of three-dimension radiation on the surface, boundary-layer properties and clouds, the radiative scheme is coupled to a Large Eddy Simulation and to a landsurface model that mimics the response of vegetation to shortwave radiation, including its direct and diffuse components, and other environmental factors such as variations in temperature and vapor pressure deficit. We systematically compare the results of the three-dimensional (3D) radiation experiment to an identical same simulation, but with the one-dimensional (1D) radiative scheme.

We find the 3D experiment to provide a wider range of values for direct and diffuse radiation compared to the $1 \mathrm{D}$ scheme, more limited in the combinations of direct and diffuse radiation at the surface. The 3D experiment differs from $1 \mathrm{D}$ after 13 Local Time (LT), i.e. 7 hours after sunrise and about 3 hours after cloud onset. After 13 LT the upper half of the sub cloud layer shows enhanced turbulence kinetic energy, almost $50 \%$ larger, and turbulent fluxes. These leads to a more moist cloud layer and further growth of turbulence within that layer during the afternoon. After 15 LT the tilting of the cloud shades adds to the differences between $1 \mathrm{D}$ and $3 \mathrm{D}$ experiments, with stronger updrafts within the subcloud layer and further enhanced turbulence in the cloud layer. The cloud size noticeable changes depending on the radiation scheme employed: while in 1D cloud across-size stabilizes at around $300 \mathrm{~m}$ at $13 \mathrm{LT}$, in $3 \mathrm{D}$ cloud size continues growing up to $500 \mathrm{~m}$. These wider clouds also show more buoyant updrafts (larger vertical velocities) that enhance the transport of moisture. This is coherent with the more intense updrafts found in the subcloud layer. These results demonstrate the limitations of $1 \mathrm{D}$ radiative schemes typically used in cloud-resolving models and their physical implications in the boundary layer and cloud dynamics. 


\subsection{Introduction}

The presence of boundary layer clouds and its interaction with radiation causes two main effects in the atmospheric boundary layer (ABL) during the day: in the atmosphere, the net effect of clouds on solar and thermal radiative fluxes modify the atmospheric heating rates and, thus, the air temperature and the related moisture capacity; at the surface, the shading and light reflections due to clouds create dynamic heterogeneities. The coupling of these rapid surface variations with the atmosphere, in the order of minutes and 100 meters, has the potential to alter the properties of the ABL and, consequently, clouds themselves.

The atmospheric heating rates due to radiation, part of the atmospheric radiative transfer problem, can be simulated with a very high accuracy using three dimensional radiative transfer models (Cahalan et al., 2005). Yet their intensive computational cost make such methods inviable for numerical experiments addressing the interactions of radiation with other processes, such as atmospheric dynamics or vegetation processes. To circumvent this demand, current efforts are directed towards providing a sufficiently fast and accurate approximation of the solution to the three-dimensional radiative transfer to be used on cloud resolving models such as Large Eddy Simulation (LES) (Jakub and Mayer, 2015).

The second effect, i.e. the response of surface to dynamic heterogeneities created by cloud-radiative interactions, is currently a matter of study in the atmospheric modeling community. The first study on this issue, with several limitations on cloud dynamic and land-surface model was the one by Schumann et al. (2002). Yet they were able to describe the updraft weakening or strengthening of updrafts due to the solar-angle dependant shade location, and reported shading to reduce the spatial scales in the ABL. Horn et al. (2015) simulated by means of LES a shallow cumulus day with typical Amazonian conditions and found the shading of clouds to reduce the size and lifetime of updrafts, i.e. the main mechanism for transport of heat and moisture to the clouds. Consequently, the size of clouds diminished, although the cloud cover remained unchanged. They also described the appearance of counterflows from shaded to sunny areas at the surface in the ABL due to the temperature gradients generated by the surface shading, weakening the cloud development. Gronemeier et al. (2016) found such circulations to strengthen the cloud development when solar angle was high. The previous studies, however, made use of a one-dimensional radiative transfer model, with the related strong limitations allowing radiation to travel only vertically (Horn et al., 2015) or along a tilted column (Gronemeier et al., 2016). Jakub and Mayer (2017) pioneered by accurately modeling for the first time a shallow cumulus case on LES combined with a relatively fast three dimensional radiative transfer model. They employed a 4-layer land-surface model providing surface fluxes based on net radiation, wind and soil properties. They found surface effects of cloud- 
radiation interactions to dominate over the impact of clouds on atmospheric heating rates. Furthermore, they showed formation of cloud streets without wind and as a consequence of the three dimensional projection of the incoming radiation at the surface. Under wind conditions, they found that depending on the relative direction of the wind with respect to the solar angle three-dimensional radiation could further reinforce the cloud street-wise patterns.

Modifications of incoming shortwave radiation have a direct impact on the vegetated surface. It is known that the available radiation and its ratio between direct and diffuse influences the photosynthesis by vegetation (Katul et al., 2012; Kanniah et al., 2012). The latter couples the surface fluxes and $\mathrm{CO}_{2}$ through the water and carbon exchange of leaves. Thus, the character and distribution of radiation at the surface will certainly alter the surface fluxes if vegetation responses are taken into account. For example, whether the cloud-surface processes described by Schumann et al. (2002); Horn et al. (2015); Gronemeier et al. (2016) will hold remains unclear. The case with three-dimensional radiation may bring numerical studies closer to observations (Zhang and Klein, 2013) compared to common one-dimensional simulations. In fact, it is particularly interesting given the large differences at the surface direct and diffuse radiation as reported by Veerman (2019). Min and Wang (2008) demonstrated that vegetation responds to clouds not only due to its radiative reduction and direct-diffuse ratio, but also due to the related colder temperature and therefore modifications in the vapor pressure deficit. Gu et al. (2002) further corroborated this by analyzing measurements at five different vegetation types. These changes in vegetation performance may feed back to the atmosphere. Demonstrating the further potential of vegetation to alter surface fluxes and cloud characteristics, Vilà-Guerau de Arellano et al. (2014) found reduced cloud cover but more active clouds over the more water-efficient C3 grass compared to C4 grass. Pedruzo-Bagazgoitia et al. (2017) showed that latent heat flux and carbon uptake is enhanced under thin clouds due to an optimal increase of diffuse radiation, but its occurrence was too low to feed back on ABL properties. These enhanced fluxes under thin clouds, however, disappear when wind is present (Sikma et al., 2018) or when using a three-dimensional radiation scheme (Veerman, 2019). Additionally, Veerman (2019) showed that using 3-D radiation in a mid-latitude case increases surface flux variability and their spatial scales, leading to thicker clouds and potentially deep convection.

Here, we aim to fill the knowledge gap and investigate the role of $3 \mathrm{D}$ versus $1 \mathrm{D}$ radiation and the related surface responses and whether these drive different ABL dynamics and, consequently, cloud properties. To that end we simulate a shallow cumulus case based on Amazonian dry season conditions. Our research gains relevance there given the strong footprint of vegetation in the region (REF). In addition, the Amazonian dry season is characterized by locally-generated deep convection (Zhuang et al., 2017) where vegetation plays a critical role (Wright et al., 2017). Thus, the results may shed light on the shallow to deep convection transition and how it is currently represented by models. 


\subsection{Methods}

We perform the numerical experiments using the version 4.2 of the Dutch Atmospheric Large Eddy Simulation (DALES) (Ouwersloot et al., 2016; Heus et al., 2010). This LES resolves most of the turbulent motions within the atmospheric boundary layer, including the explicit dynamics of clouds. Of relevance in our study is the radiation schemes used. To take full advantage of the radiative features, we employ an interactive and surface scheme sensitive to radiation as well as other environmental variables. Note that while the dynamics are explicit in our experiments, the vegetated surface response and radiation are parameterized to a certain degree by the land-surface model and radiative transfer scheme, respectively. We describe below the most relevant features of each physical process representation.

\subsubsection{Radiation schemes: $1 \mathrm{D}$ and 3D}

The two radiative schemes employed to resolve the radiative transfer of both shortwave and longwave radiation differ in several features. The main difference lies on the spatial dimensions in which radiation is allowed to travel: one dimension, for the Rapid Radiative Transfer Model for GCMs (RRTMG, Iacono et al. (2008)) and three dimensions, for TenStream (Jakub and Mayer, 2015). RRTMG is a relatively fast radiative transfer model dividing the shortwave and longwave spectra into 14 and 16 spectral bands, respectively, and computing all the bands at every radiation timestep. It only allows for the vertical transfer of radiation, thus hindering any radiation transfer in the horizontal directions. Such a scheme is typically referred as a two-stream (upwards and downwards) radiative model.

In turn, TenStream consists of 13 streams along which radiation is allowed to travel: 3 streams for direct radiation, i.e. downwards and sidewise in each horizontal direction, and 10 streams for diffuse radiation, i.e. 2 vertical, and 4 diagonal streams for each horizontal direction (Jakub and Mayer, 2015). It uses the same RRTMG to derive the optical properties such as optical depths, single scattering albedo and asymmetry parameter, thus minimizing the differences between schemes. Being a much more accurate radiative transfer scheme than two-stream models taking more sophisticated and computationally expensive Monte Carlo methods (Jakub and Mayer, 2015; Veerman, 2019) as reference, TenStream is computationally one order of magnitude more demanding than typical one-dimensional radiative transfer models (Jakub and Mayer, 2015). To reduce the computational time adaptive spectral integration is used, i.e. radiative bands are only updated once significant differences in their properties have taken place. All the spectral bands are simultaneously updated at a fixed coarser time resolution. 


\subsubsection{Interactive land-surface scheme}

We use the A-gs, i.e. Assimilation-stomatal conductivity, land-surface model incorporated in DALES 4.2 (Jacobs and de Bruin, 1997; van Heerwaarden et al., 2010; PedruzoBagazgoitia et al., 2017). This land-surface model mimics the behavior of vegetation based on plant physiology, regulating in this way the surface turbulent fluxes and coupling them to the plant $\mathrm{CO}_{2}$ uptake through photosynthesis. The activity of vegetation and thus, exchange rates between the surface and atmosphere is sensitive to several environmental variables known to affect photosynthesis rates, among which: the available soil moisture, the near surface-wind through the aerodynamic resistance, vapor pressure deficit, amount of direct and diffuse shortwave radiation, temperature and atmospheric $\mathrm{CO}_{2}$ concentration. Due to the dependence of surface fluxes on direct and diffuse shortwave radiation, the use of a one-dimensional or three-dimensional radiative scheme has vast consequences on the surface fluxes. These will be shown in Fig. 5.1.

\subsubsection{Experiments and numerical settings}

We base our study on a shallow cumulus case following typical conditions on the Amazonian region during its dry season. The initial conditions are based on a full month of observations obtained during the GoAmazon2014/2015 campaign, thus representing an aggregate case of typical dry Amazonian conditions. The dry season is a transitional period in the Amazon, where the vegetated surface may alter local conditions and locally trigger deep convection (Wright et al., 2017). A full characterization of the LES case, including an exhaustive set of observations for validation and an additional ECMWF high resolution simulation is presented by Vilà-Guerau de Arellano et al. (2019).

The initial potential temperature $\theta$ profile at 6 local time (LT) is characterized by three distinct layers: a stable lowermost layer with a warming of $6 \mathrm{~K}$ between the surface, at $297 \mathrm{~K}$ and the $500 \mathrm{~m}$ level, a more unstable layer between $500 \mathrm{~m}$ and $2000 \mathrm{~m}$ with a $2.45 \mathrm{~K} \mathrm{~km}^{-1}$ lapse rate, and an uppermost more stable layer above warming at an approximate rate of $5 \mathrm{~K} \mathrm{~km}^{-1}$. The specific moisture $q_{t}$ at the surface is of $16.6 \mathrm{~g}_{\mathrm{w}} \mathrm{kg}_{\mathrm{a}}^{-1}$ with constant $-2.96 \mathrm{~g}_{\mathrm{w}} \mathrm{kg}_{\mathrm{a}}^{-1} \mathrm{~km}^{-1}$ drying up to the top of the domain. In contrast with the case described in Vilà-Guerau de Arellano et al. (2019) our simulations are performed without horizontal wind. The motivation for this change is the aim of focusing on the pure effect of cloud radiative impacts both at the surface and on the ABL dynamics, while wind is known to homogenize the radiative-driven differences due to clouds and its effects (Horn et al., 2015; Sikma et al., 2018).

We employ a $21.6 \times 21.6 \times 5 \mathrm{~km}^{3}$ domain with a gridbox size of $100 \times 100 \times 20 \mathrm{~m}^{3}$, periodic boundaries in the horizontal directions and an adaptive time-stepping with a maximum of $20 \mathrm{~s}$. The only difference between the two numerical experiments is the radiation scheme used. Experiment 1D uses the one-dimensional RRTMG radiative scheme, while 
experiment 3D uses the TenStream scheme. In the latter, a minimum solar zenith angle of $7^{\circ}$ is observed at $1200 \mathrm{LT}$, and values above $20^{\circ}$, described by Jakub and Mayer (2017) as situations where the 3D effects of radiation may be larger, take place before $10: 45$ and after $13: 15 L T$. As explained in Sect. 5.2.1 radiation calls are carried differently depending on the scheme used: in 1D RRTMG is called every 30 seconds, while in 3D TenStream the changing bands are updated every 30 second while a total update of all bands is performed every 5 minutes. Such high temporal resolution is required in order to provide the surface with the immediate impact of the rapidly changing shallow clouds. For this same reason the land-surface model is called at every timestep. A simple all or nothing microphysical scheme is used in which $q_{l}=q_{t}-q_{s a t}$ if $q_{t}>q_{s a t}$ and $q_{l}=0$ otherwise, where $q_{l}$ is the liquid water mixing ratio, and $q_{\text {sat }}$ the specific humidity at saturation.

\subsubsection{Spatial autocorrelation function and length scales}

In Sect. 5.3.3 we characterize the average horizontal dimensions of clouds in both directions $x$ and $y$ by the length scales $\lambda_{x}$ and $\lambda_{y}$, respectively. Following the work by Horn et al. (2015), we define:

$$
\lambda_{x}=\int_{0}^{\rho^{L x}} \rho_{x}^{L}\left(L_{x}\right) d L_{x}
$$

where $L_{x}$ is the shift in the horizontal direction $x$ and $\rho^{L_{x}}$ the autocorrelation function in space along $x$ direction. The definition of $\lambda_{y}$ is analogous to Eq.5.1. $\rho^{L_{x}}$ expresses the probability of finding at a distance $L_{x}$ properties similar to the ones at the point of origin. Since our purpose is to study the cloud size, we take liquid water path (LWP) as the variable on which we apply the autocorrelation function.

\subsection{Results}

\subsubsection{Enhanced variability of surface responses}

The unidirectional character of light on a 1-D scheme limits the variability of radiation at the surface (Veerman, 2019), and the surface response. To quantify that, Figs. 5.1a,b show the frequency of latent heat flux LE values for given direct SWdir and diffuse shortwave radiation SWdif at the surface, respectively, between 11:30 and 12:30 LT. The limited variability of the 1-D simulation, and thus, the almost uniquely defined relation between direct and diffuse radiation (Veerman, 2019), allows to travel along the curves as a surface response to the life-cycle of individual clouds: surface points under clear sky fall at the yellow areas in Figs. 5.1a,b, and the onset of a shallow cloud shifts the point to the left (right) towards lower (higher) direct (diffuse) radiation until a maximum of about 

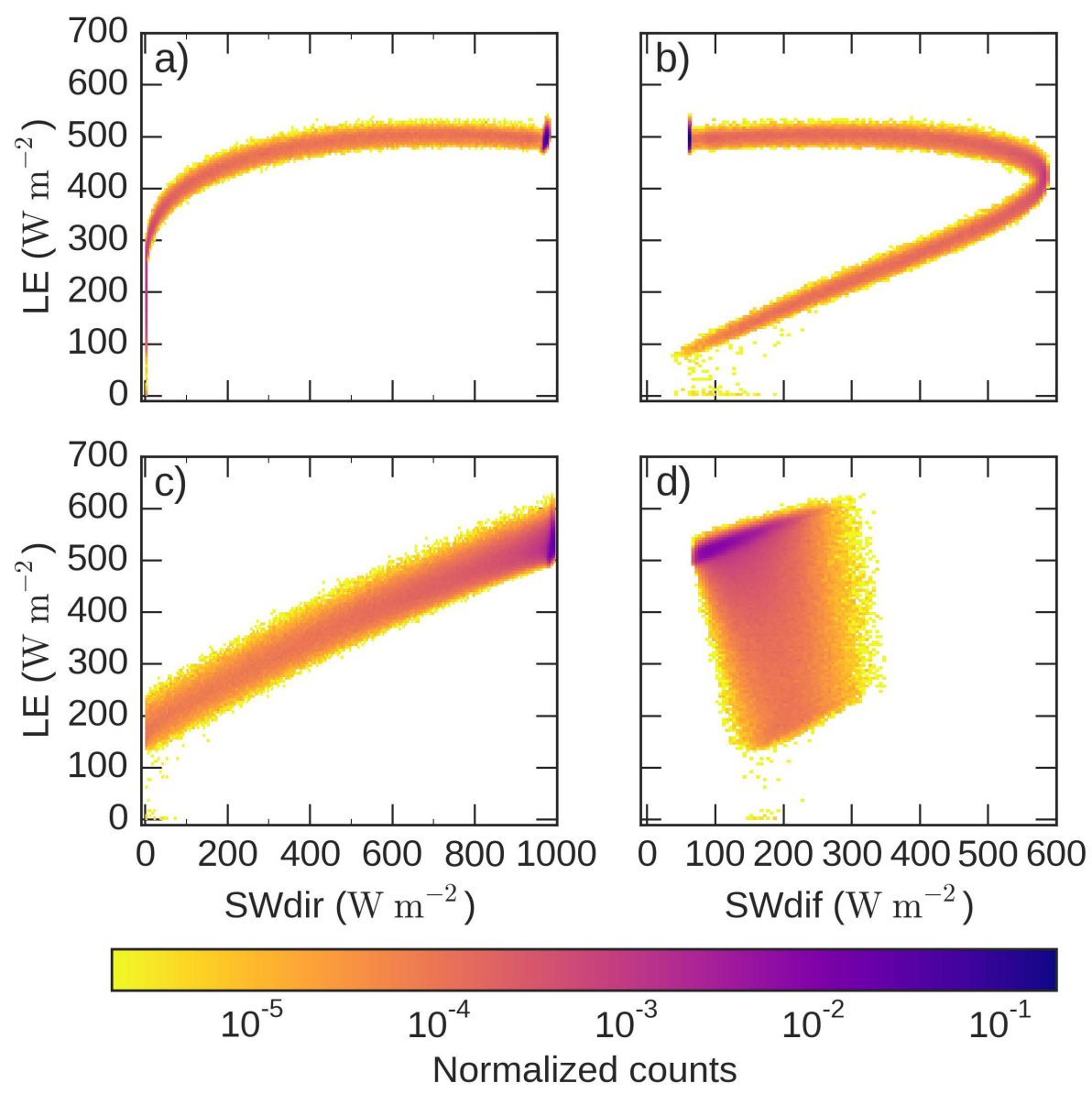

Figure 5.1: Probability density function of the indicated latent heat flux LE and shortwave direct radiation SWdir (left) and LE and shortwave diffuse radiation SWdif (right) for the 1D (top) and 3D (bottom) simulations. The counts are binned every $5 \mathrm{~W} \mathrm{~m}^{-2}$ and include all the values for the whole domain in each experiment at a 1 minute temporal resolution between 11:30 and 12:30 LT.

$600 \mathrm{~W} \mathrm{~m} \mathrm{~m}^{-2}$ in diffuse radiation is reached. Along this range the diffuse fertilization effect by thin clouds is possible. In other words, the small decrease in total downwards shortwave radiation at the surface SWd but large increase in SWdif with a higher penetration in the canopy, may lead to enhanced photosynthesis and, thus, larger $\mathrm{CO}_{2}$ assimilation and latent heat flux at the surface (Pedruzo-Bagazgoitia et al., 2017) than under clear sky conditions. The further growth of a cloud leads to a reduction of both direct and diffuse radiation, thus descending along the curves towards lower LE. Solving the radiative transfer using a 3D approach presents a much broader range of values for the surface response for given SWdif (SWdir) (Veerman, 2019), with a variability of up to 500 (100) $\mathrm{W} \mathrm{m} \mathrm{m}^{-2}$ on LE, while it was less than $100(50) \mathrm{W} \mathrm{m}^{-2}$ in $1 \mathrm{D}$. This is due to the fact that the surface values at a certain point depend not only on the column above as in $1 \mathrm{D}$, but on all other directions as well. As a consequence, diffuse radiation is more homogeneously distributed along the domain in 3D: all points at the surface show $\mathrm{SWdir}<350 \mathrm{~W} \mathrm{~m} \mathrm{~m}^{-2}$, 

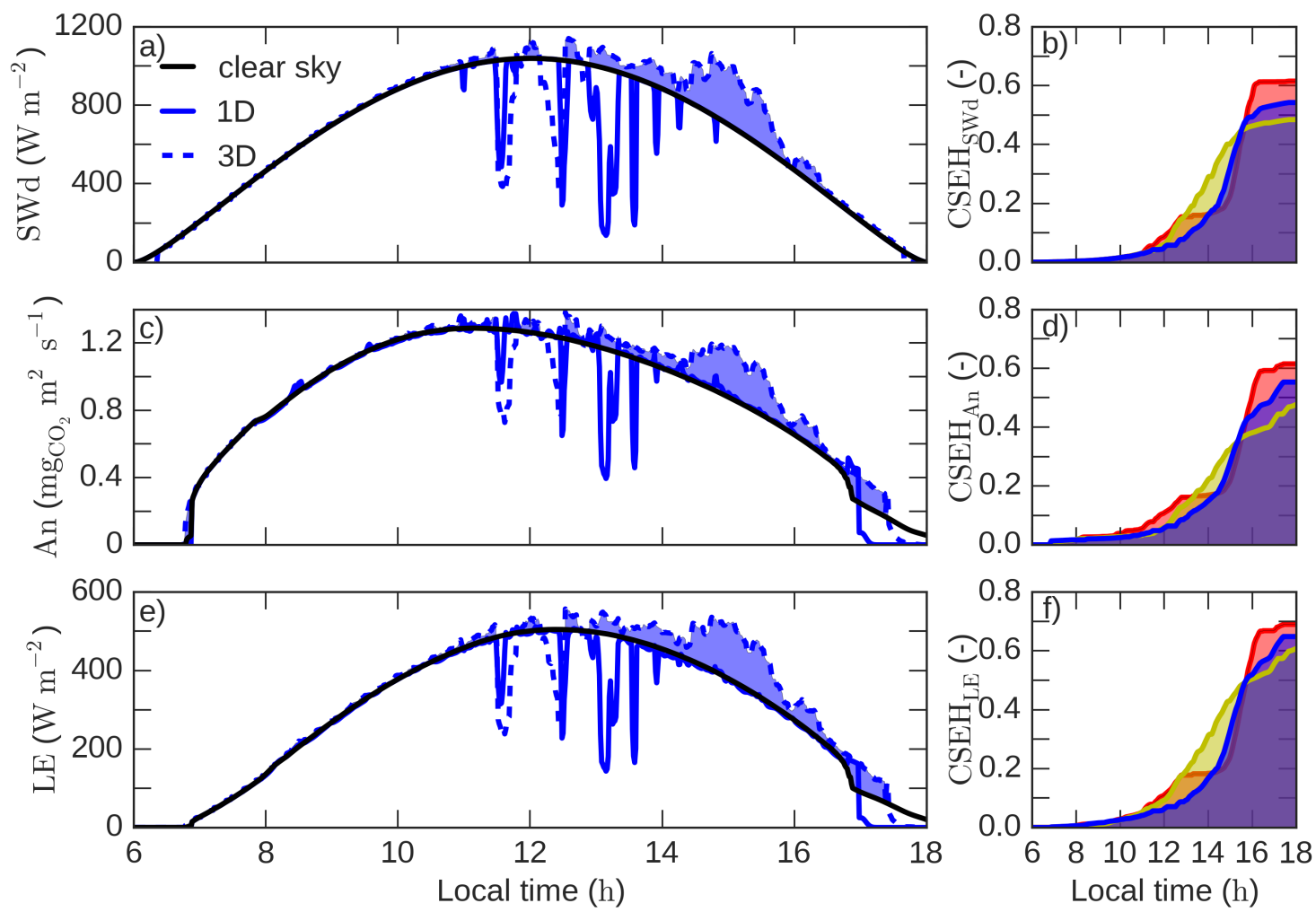

Figure 5.2: On the left, time series of downwards shortwave radiation at the surface SWd (top), vegetation net carbon uptake An (center) and latent heat flux LE (bottom) of a chosen point in 1D (full blue line), 3D (dashed blue line) experiments and clear sky reference (black line). Shaded areas indicate larger than clear sky values due to three dimensional effects of radiation in $3 \mathrm{D}$. On the top right, time series of accumulated $\mathrm{SWd}$ due to larger than clear sky values of the selected point (blue) and two additional randomly selected points (red and yellow). Values are expressed in clear sky equivalent hours (CSEH), equivalent to the clear sky SWd during the hour of maximum irradiance, i.e. between 11:30 and 12:30 LT. The analogous is shown at center right and bottom right for An and LE, respectively.

while for $1 \mathrm{D}$ values up to $580 \mathrm{~W} \mathrm{~m} \mathrm{~m}^{-2}$ were under some clouds.

Besides a larger range of surface values, the three-dimensional character of radiation produces new features at the surface. The global downwards shortwave radiation SWd under clear sky follows a sinusoidal-like shape, peaking around noon due to maximum solar irradiance. The curve in $1 \mathrm{D}$ follows that shape with drops after $11 \mathrm{LT}$ related to the presence of clouds above (Fig. 5.2a). In contrast, the three dimensional effects of clouds produce not only negative fluctuations in SWd but also peaks larger than clear sky values. These peaks are due to the reflection of light on the sides of clouds that is directed towards the surface. The additional energy by such effects in the 3D simulations reaches the equivalent to $60 \%$ of the clear-sky SWd arrived during the most intense hour of the day, i.e. between 1130 and 1230 LT. These additional radiation at the surface 

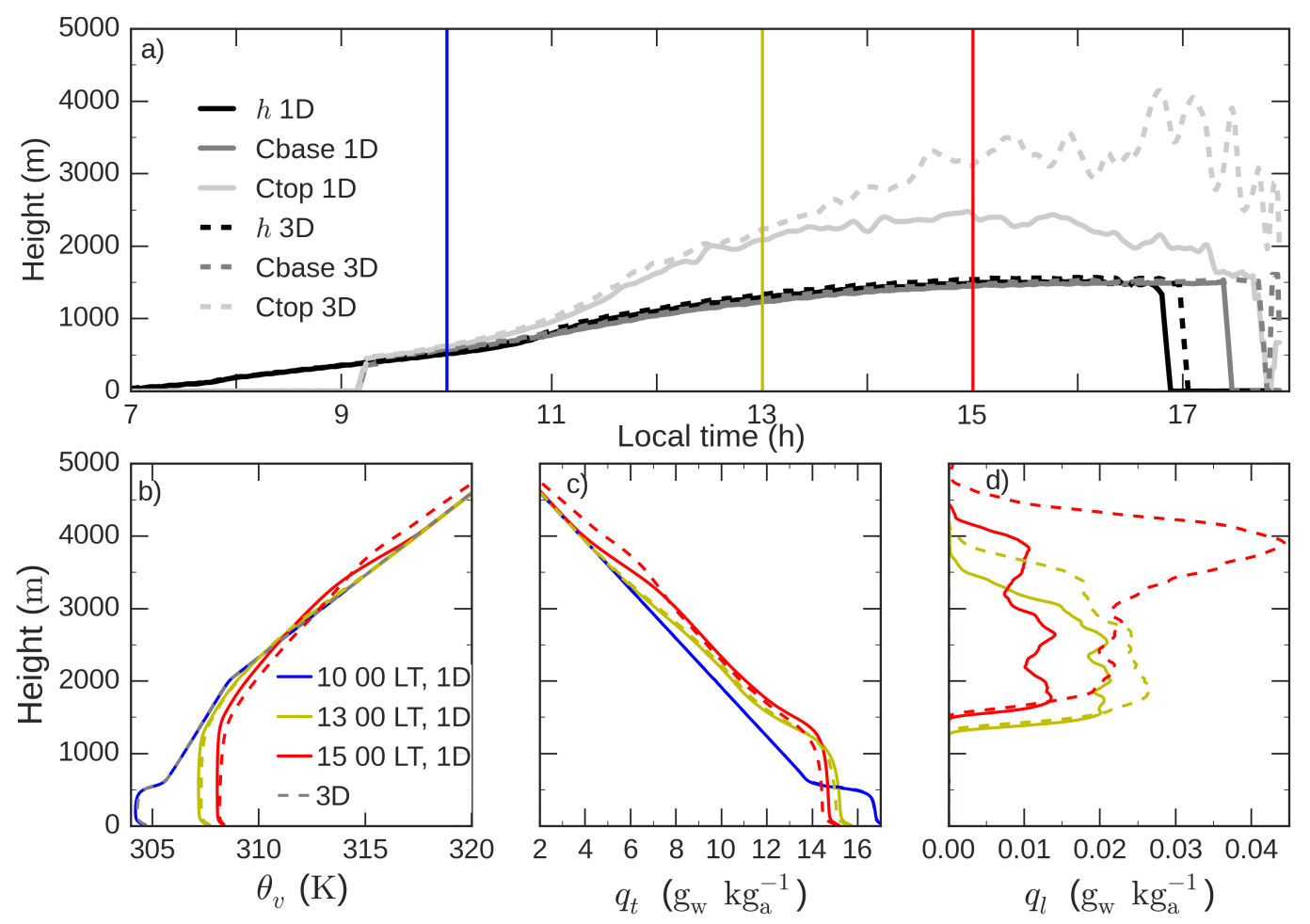

Figure 5.3: On top, time series of boundary layer height $h$ (black), minimum cloud base height Cbase (dark grey) and average cloud top height Ctop (light grey) for 1D (full lines) and 3D (dashed lines). Below, vertical profiles on 1D and 3D of slab averaged virtual potential temperature $\theta_{v}$ (left), total specific moisture $q_{t}$ (center) and liquid specific moisture $q_{l}$ (right) at 1000 (blue), 1300 (yellow) and 1500 (red) LT.

drives larger photosynthesis rates of the vegetation and, thus, larger net $\mathrm{CO}_{2}$ absorption An and water vapor release, enhancing the surface latent heat flux (see Figs. 5.2c,e). The accumulated effect on An and LE during the day reaches values between $60 \%$ and $70 \%$ of the respective clear sky equivalent hour (CSEH). Despite these effects the reader should note that the net effect of clouds on surface SWd is still negative, but smaller in magnitude than in $1 \mathrm{D}$.

\subsubsection{Thermodynamic and turbulent effects}

The three-dimensional effects of radiation barely impact the atmospheric state before and during the first hours after cloud onset. This is shown in Fig.5.3, where the boundary layer height $h$, defined as the height of buoyancy flux minimum, the lowest cloud base and the mean cloud top coincide among experiments until 1300 LT. Similarly, the ABL remains well mixed and with similar virtual potential temperature $\theta_{v}$ and specific moisture $q_{t}$ values (Figs.5.3b,c). The lowest cloud base follows the ABL height as expected in cumulus-topped boundary layers (REF). Between 13 and 16 LT ctop in 3D grows reaching 
differences of up to $1000 \mathrm{~m}$ with 1D. By 13 LT the cloud layer in 3D shows enhanced liquid water mixing ratio $q_{l}$ by about $0.07 \mathrm{~g}_{\mathrm{w}} \mathrm{kg}_{\mathrm{a}}^{-1}$ compared to $1 \mathrm{D}$, suggesting the presence of more or thicker clouds. This will be confirmed in Fig. 5.5. At 15 LT the trend is more obvious and 3D presents values as high as $0.045 \mathrm{~g}_{\mathrm{w}} \mathrm{kg}_{\mathrm{a}}^{-1}$ at $4000 \mathrm{~m}$. After 15 LT ctop in 1D gradually decreases until clouds disappear at 1730 LT. In 3D, however, ctop continues its growth after 15 UTC reaching $4000 \mathrm{~m}$ and persisting after the collapse of the ABL. This is a consequence of previous cloud activity, more intense than in 1D (see Fig. 5.3) and preconditioning a moister and more unstable lower troposphere (see 1500 LT profiles in Fig.5.3b,c,d). This moistening is necessary for the transition from shallow to deep convection (Zhuang et al., 2017; Zhang and Klein, 2010). Such conditions allow further growth of clouds after the collapse of the ABL even with very weak surface forcings, and reveal the underestimation of the potential for shallow-to-deep convection transitions by one-dimensional radiative schemes. These will be further studied in Sect. 5.3.3. Note that although average cloud top peaks at $4000 \mathrm{~m}$, few clouds reach the domain top at $5000 \mathrm{~m}$. These may question the reliability of the results after 15 UTC. However, the inertia of the clouds reaching the domain top is generally weak (see Figs. 5.4 and 5.6), partly due to the sponge layer dampening perturbations near the domain top, and the top of domain horizontal cloud fraction never reaches values above 0.03 . Thus, we assume that the domain top has little impact on the simulation.

The different treatment of radiation coupled to the surface has a direct impact on the turbulent transport fields once clouds are present. The turbulent structure of the subcloud and cloudy layer is affected by the radiation, mostly due to the displacement of the shading below the cloud (Jakub and Mayer, 2017). The two relative maxima present in the turbulence kinetic energy (TKE) (Fig. 5.4a) are representative of a cumulus cloudtopped boundary layer with the first maximum at about $\frac{h}{2}$ and the second within the cloud layer (REF). The larger TKE in the upper half of the subcloud layer, i.e. between 500 and $1000 \mathrm{~m}$, for 3D at $13 \mathrm{LT}$ shows enhanced turbulence in 3D also maintained along the cloud layer. The coinciding skewness for both experiments at 13 LT suggests that the turbulent structure is similar within the subcloud layer. At $15 \mathrm{LT}$, the subcloud TKE shows a different shape for $1 \mathrm{D}$ and 3D but with a similar subcloud maximum. Yet the large differences in skewness show that the turbulence in 3D is generated by much stronger updrafts with higher positive vertical velocities. In addition, the updrafts reaching the lifting condensation level (LCL) intensify above $3000 \mathrm{~m}$ showing much larger skewness and TKE. Such turbulent conditions affect the transport of buoyancy and moisture (Figs. $5.4 \mathrm{c}, \mathrm{d})$. The subcloud buoyancy flux shows little impact besides a larger entrainment of tropospheric air due to the enhanced TKE. Within the cloud layer, however, 3D presents larger buoyancy transport showing $\overline{w^{\prime} \theta_{v}^{\prime}}$ values almost as high as at the surface. The reason for such larger buoyancy, apart from the stronger updrafts at subcloud layer, lies on the present moisture. Already by $13 \mathrm{LT}$ the $3 \mathrm{D}$ experiment shows larger $\overline{w^{\prime} q_{t}^{\prime}}$ : below the entrainment zone the moisture flux is $10 \%$ larger in $3 \mathrm{D}$ than in $1 \mathrm{D}$, and such differ- 

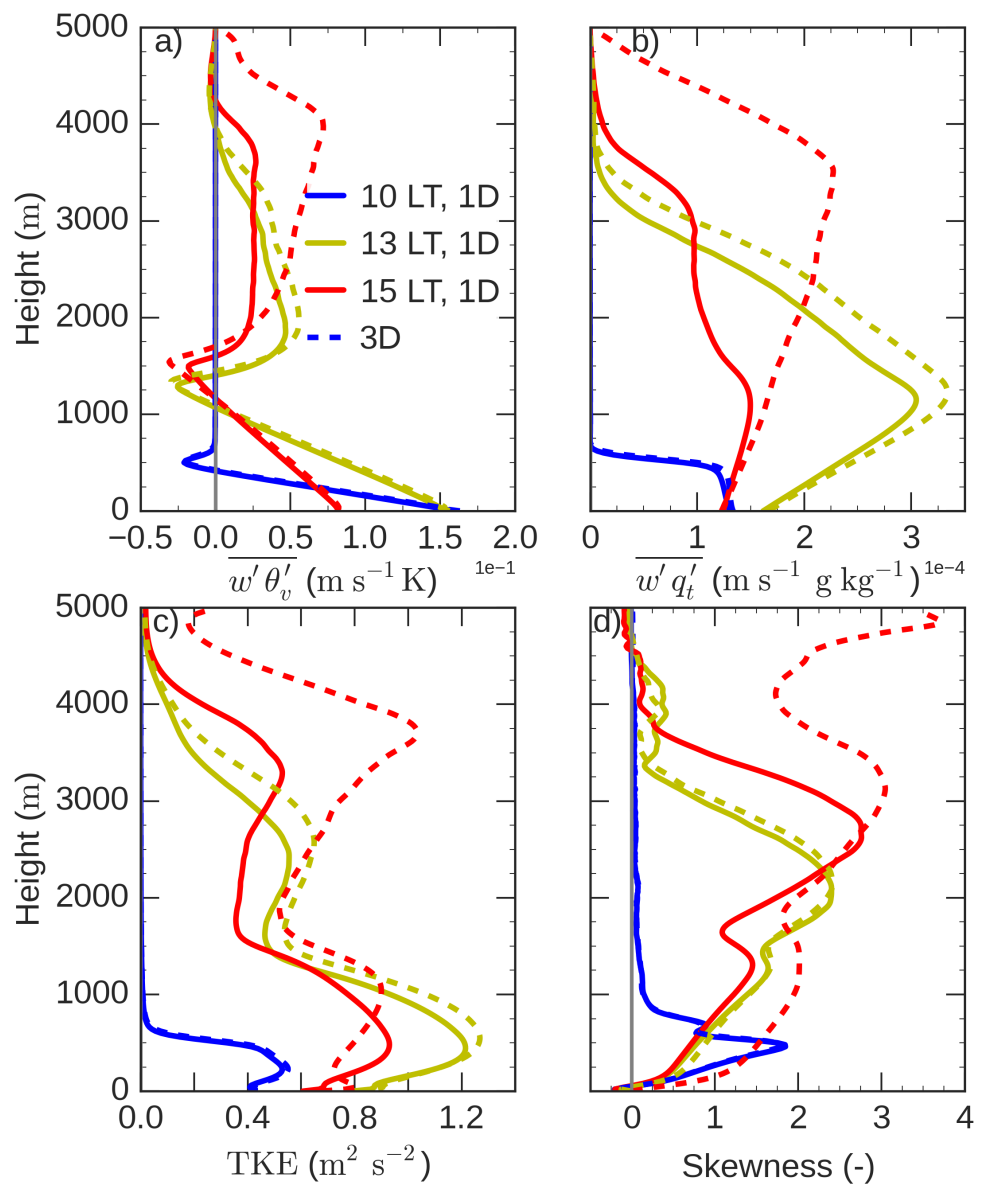

Figure 5.4: Vertical profiles of slab average turbulent buoyancy flux $\overline{w^{\prime} \theta_{v}^{\prime}}$ ( top left), specific moisture flux $\overline{w^{\prime} q_{t}^{\prime}}$ (top right), turbulence kinetic energy (lower left) and vertical velocity skewness $\frac{\overline{w^{\prime 3}}}{\overline{w^{\prime 2}} \frac{3}{2}}$ (lower right) at 1000 (blue), 1300 (yellow) and 1500 (red) LT. Full and dashed lines show $1 \mathrm{D}$ and $3 \mathrm{D}$ results, respectively.

ence remains within the cloud layer. The additional moisture transported on average to the cloud layer in 3D hampers cloud dissipation locally as the air entrained into clouds is less dry than the initial free troposphere. In addition, the suggested intensification of the dynamic heterogeneities (Jakub and Mayer, 2017; Veerman, 2019) could potentially moisten the environment around clouds further weakening the drying by lateral entrainment. Veerman (2019) showed that surface fluxes were increased below clouds due to enhanced diffuse radiation, and that lead to larger dominating scales in the ABL. Based on the results shown above we conclude that while the amount of turbulence of the subcloud layer remains similar, the updrafts are stronger in 3D, specially after 13 LT. This, together with a more moist cloud layer, allows for more vigorous cloud development in $3 \mathrm{D}$. 

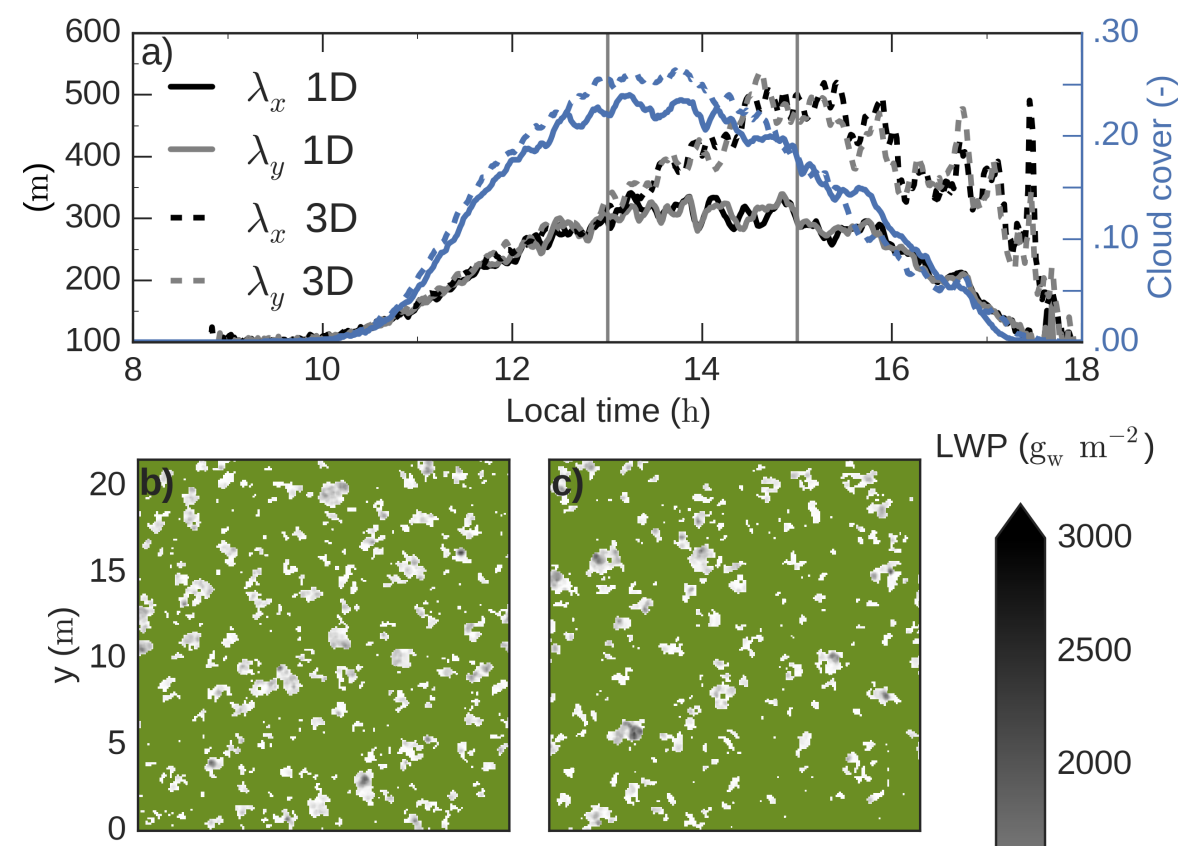

$\operatorname{LWP}\left(\mathrm{g}_{\mathrm{w}} \mathrm{m}^{-2}\right)$
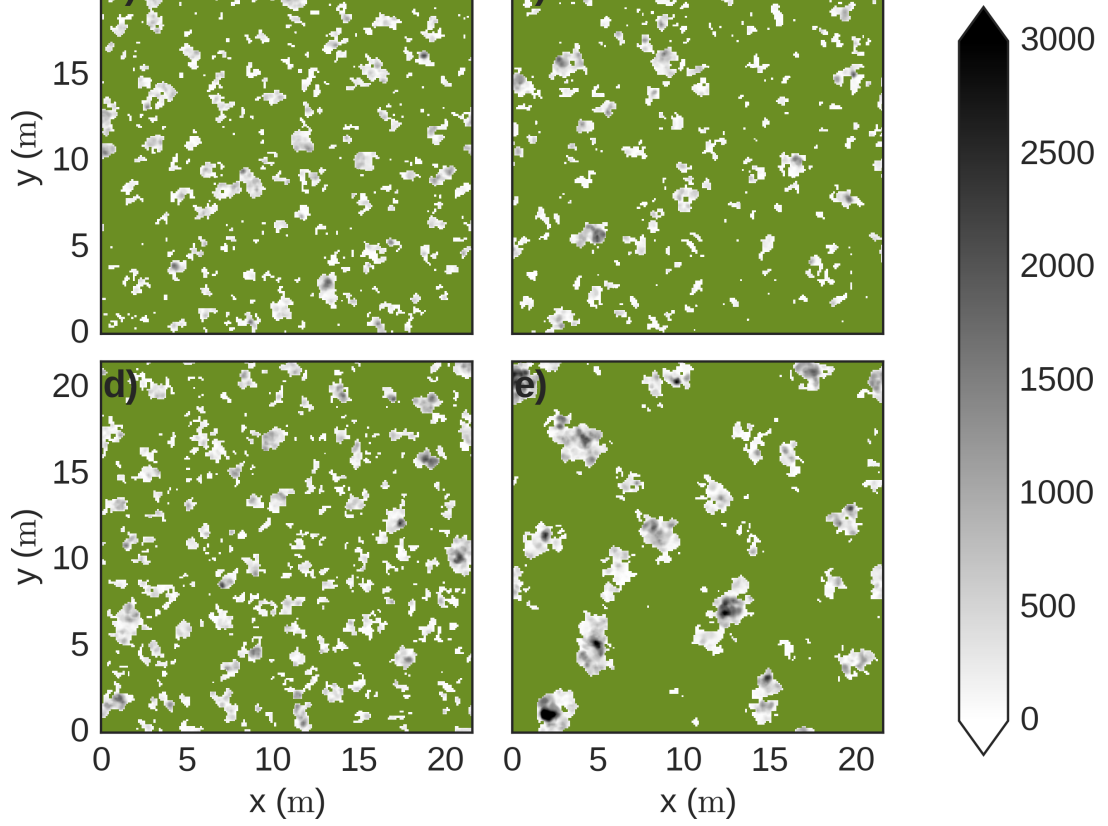

Figure 5.5: On top, time series of cloud length scale in both horizontal directions $\lambda_{x}$ and $\lambda_{y}$ (black and grey lines, respectively) and cloud cover (blue lines) for 1D (full lines) and 3D (dashed lines). Below, cenital view of liquid water path LWP at 13 LT (left) and 15 LT (right), as indicated by the vertical grey lines in a), for $1 \mathrm{D}(\mathrm{b}, \mathrm{c})$ and $3 \mathrm{D}(\mathrm{d}, \mathrm{e})$. Note that the lowest threshold of $100 \mathrm{~m}$ in $\lambda_{x}$ and $\lambda_{y}$ is due to the horizontal resolution of our experiments.

\subsubsection{Modified cloud structure and dynamics}

To reveal whether the stronger convection impacts cloud characteristics we investigate how the three dimensional treatment of radiation affects the size and spatial structure of the clouds in Fig.5.5. To that end we characterize the clouds by quantifying the cloud cover and the horizontal size of the clouds by the length scales $\lambda_{x}$ and $\lambda_{y}$ as described in Sect. 5.2.4. We find the cloud length scales to be very similar in their $x$ and $y$ directions for both $1 \mathrm{D}$ and 3D, showing that there is no preferred direction for cloud growth. This contrasts with the findings by Jakub and Mayer (2017), who found the solar angle to influence the orientation and alignment of clouds with three-dimensional radiation. In 
their case, however, they fixed the solar angle, while in the experiments presented here the zenith angle at noon is low given its low latitude, and the solar angle varies along the experiment following the diurnal cycle. The different results using three-dimensional radiation suggest that the timescales required for cloud alignment are longer than those related to the relative movement of the sun and its response by the ABL dynamics. Until 13 LT we observe a very similar quasi-linear growth of the cloud length scales for both $1 \mathrm{D}$ and 3D, consequent with the coinciding cloud base and top development found in Fig.5.3. During that time, cloud cover grows at a similar rate for both experiments. Such a linear growth was also found by Rieck et al. (2014) at least for the largest clouds in their simulations. Afterwards, $\lambda_{x}$ and $\lambda_{y}$ in $1 \mathrm{D}$ plateau at $300 \mathrm{~m}$ for about two hours. One hour earlier, the cloud cover oscillates between 0.20 and 0.25 . Such behavior suggests a quasi-equilibrium state with clouds bi-directionally coupled to the surface described as follows (Vilà-Guerau de Arellano et al., 2014): first, clouds form once the warm moist updrafts rising from the surface reach the LCL and condensate. The maintenance of the clouds is, thus, dependent on the surface and the supply by the updrafts below. This state reveals the link from surface to the clouds. Second, clouds grow until a critical thickness is reached. Then, the thickness-related shading reduces the surface fluxes under the individual clouds, hampering their further development by weakening the updrafts below. This state shows the bidirectional coupling through the appearance of shading as the way in which clouds impact surface. The weaker updraft hampers the maintenance of the cloud accelerating its dissipation and favors the appearance of new clouds at other locations, maintaining the cloud cover over the domain nearly constant. Such quasiequilibrium in cloud cover has been found in other simulated shallow cumulus cases both near the tropics (Horn et al., 2015) and mid-latitudes (Vilà-Guerau de Arellano et al., 2014; Rieck et al., 2014) and observed in mid-latitudinal forced shallow cumulus days, although not so clearly in buoyantly active ones (Zhang and Klein, 2013). The cloud cover leveling off at 13 LT suggests that the cloud critical thickness is reached at around that time.

The cloud cover behaves similarly in 3D with oscillations around 0.25 between 12:30 and 14 LT. However, the cloud size continues growing at the same rate as before 13 LT. We hypothesize this is due to two reasons: the first, is the fact that after $1315 \mathrm{LT}$ the zenith angle is larger than $20^{\circ}$, enough to displace the shade from below the clouds in 3D. Thus, the coupling between clouds and surface is broken due to the shading displacement (Schumann et al., 2002; Jakub and Mayer, 2017): the shading of the cloud does not directly impact the updrafts below, thus allowing for the maintenance of strong updrafts and further growth of the already existing clouds. This result coincides with the one by Jakub and Mayer (2017), who reported cloud-radiative feedbacks to potentially trigger cloud streets for solar zenith angles above $20^{\circ}$. These findings suggests a stronger impact by shading displacement at higher latitudes and, thus, higher zenith angles. The second reason for the further growth of $\lambda_{x}$ and $\lambda_{y}$ in 3D is, as shown by Veerman (2019), the 

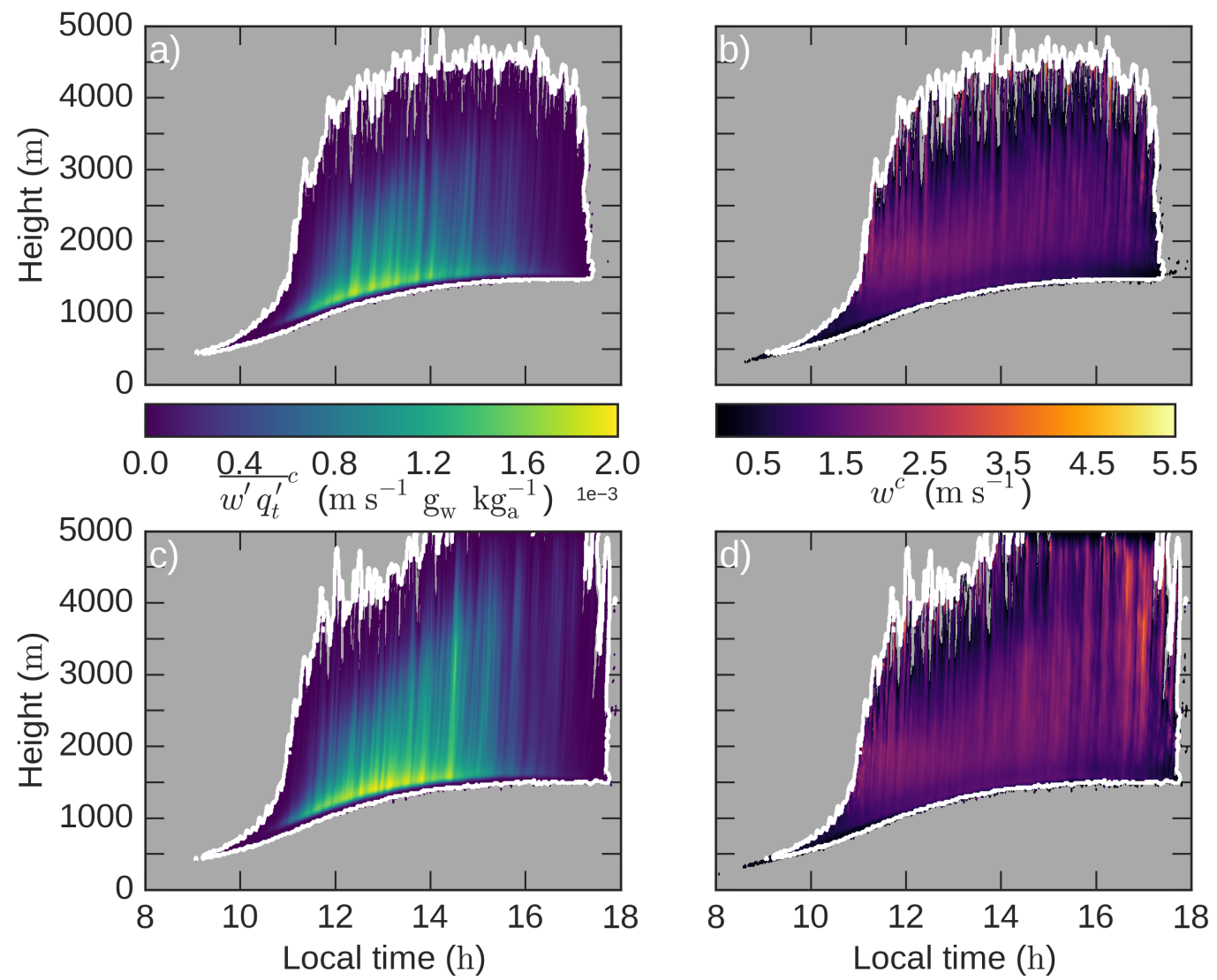

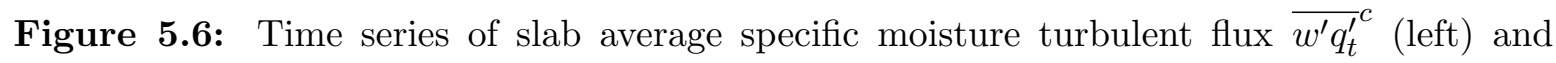
vertical velocity $w^{c}$ (right) for $1 \mathrm{D}$ (top) and 3D (bottom). Both variables are averaged only over cloudy gridboxes. The white lines show the cloud contours.

enhanced surface fluxes below already existing clouds. This would strengthen the updrafts below the already existing clouds and contribute to their further growth. The peak and further decrease of $\lambda_{x}$ and $\lambda_{y}$ after $16 \mathrm{LT}$ in $3 \mathrm{D}$ is due to the decreasing solar surface radiation.

Given the larger size of clouds, its internal dynamics are more likely to contain more vigorous updrafts and transport of subcloud layer properties to layers above. To study the internal cloud dynamic characteristics we perform the conditional average of the turbulent properties only within the cloudy gridboxes, i.e. grid boxes with $q_{l}>0$. We show in Fig. 5.6 the time series of such conditional averages for turbulent moisture flux ${\overline{w^{\prime} q_{t}^{\prime}}}^{c}$ and vertical velocity $w^{c}$. The transport of moisture for both $1 \mathrm{D}$ and $3 \mathrm{D}$ is stronger for the first levels above cloud base and weaker higher up as 1) lateral entrainment within the individual clouds dries the updrafts and 2) the turbulent motions are in general weaker as the clouds grow towards more stable layers of the atmosphere. We still find, in agreement with the total $\overline{w^{\prime} q_{t}^{\prime}}$ from Fig. 5.4b, stronger moisture transport near cloud base in 3D after 13 LT. At later stages, strong moisture transport reaches in 3D as high as $4000 \mathrm{~m}$ 
with some clouds extending up to the domain top. The vertical velocity of clouds shows continuous and large values on the lowest half of the cloud layer in 1D (Fig. 5.6b), with few isolated higher values near the top of cloud layer. The in-cloud velocities in 3D show a similar initial stage until 13 LT and afterwards, coinciding with the further growth of clouds observed in Fig. 5.2.4a, larger velocities occur along the whole cloud layer. The most extreme $w^{c}$, in the order of $5 \mathrm{~m} \mathrm{~s}^{-1}$ appear in the upper half of the cloud layer between 16 and $18 \mathrm{LT}$. Such large values at those heights, with a weak late afternoon surface forcing, may indicate the onset of deep convection by few isolated updrafts. These findings reinforce the idea of more active clouds in 3D after 13 LT, with larger velocities and transporting more moisture to the cloud layer.

\subsection{Conclusions and outlook}

We investigated the emergent features on the thermodynamics, turbulence and cloud structure and dynamics in the atmospheric boundary layer by solving the three-dimensions of the radiative transfer coupled to the surface. To that end, we performed two Large Eddy Simulation experiments with an interactive land-surface model representing vegetation responses to radiation and other environmental variables, with variations in the order of few seconds. The only difference between experiments was the one-dimensional or threedimensional character of light. We based our case on Amazonian conditions during its dry season.

The results show larger range of surface values in terms of radiation and, thus, surface fluxes when radiation is three dimensional. Subcloud layer properties remained similar during the first hours of the shallow cloud convection, except for a slightly larger turbulence kinetic energy in the upper half of the subcloud layer. Within the cloud layer, however, turbulence and the related transport of moisture showed an increase of $10 \%$, leading to a more moist and unstable cloud layer with three-dimensional radiation. Such tendency increased on time with much larger turbulence, buoyancy and moisture flux in the cloud layer leading to a larger vertical cloud development. We attributed the larger turbulence and cloud development to the fact that the cloud shading was displaced from the updraft to which the clouds are rooted, and to the increased sensible heat flux below clouds also found by Veerman (2019). This eliminated the limitation to cloud growth by the shading and subsequent sensible heat flux decrease at the surface below the cloud, typical in one-dimensional radiation experiments. As a consequence, the cloud size increased in the simulation with $3 \mathrm{D}$ radiation. The extent in both horizontal directions of individual clouds showed to increase linearly until about $300 \mathrm{~m}$, remain constant during the central hours of the day and decrease in the late afternoon for the one-dimensional radiation. In turn, employing the $3 \mathrm{D}$ radiation showed the cloud extent to further grow up to $500 \mathrm{~m}$ and remain larger than one-dimensional radiation for the rest of the experiment. 
We attributed the differences to the decoupling of the clouds and the updrafts underneath from the shading of the clouds in $3 \mathrm{D}$, and to the larger fluxes below clouds due to downwards reflection of radiation by clouds. Such decoupling leads to the formation and intensification of shallow convection and larger clouds. This findings is also supported by larger vertical velocities and moisture transport within the clouds in 3D. The findings presented here suggest that the three-dimensional treatment of radiation may be critical in the development of necessary conditions for the transition to deep convection.

From the presented work we conclude that the response of the surface to three-dimensional radiation enhances turbulence in the upper half of the boundary layer and lays the optimal conditions for deep convection. The deep convection is facilitated by the decoupling of the cloud-topped updrafts from the induced cloud shading, and the surface flux increase due to enhanced diffuse radiation below clouds. Based on these results, we present some suggestions and recommendations for further work on the topic. Firstly, the new distribution of direct and diffuse radiation at the surface presents remarkably different radiation conditions for vegetation. Thus, an analysis on the differences in $\mathrm{CO}_{2}$ absorption by vegetation, specially on larger scales, seems relevant for the assessment of the carbon cycle. Given the critical role of the updraft-shade decoupling, we expect latitudes with larger zenith angle to show a stronger and, likely, earlier divergence of 3D experiments from $1 \mathrm{D}$.

The transport of moisture by updrafts to the cloud layer aided in the development of deeper clouds. In particular, increased moisture transport and related deep convection was found to be related with larger horizontal variability within the boundary layer in observations (Zhang and Klein, 2010). Thus a further analysis on the inhomogeneities within the boundary layer, specially during the first hours after cloud onset, would aid in confirming such relation. A first step could involve the analysis of temperature or moisture covariances $\overline{\theta_{v}^{\prime 2}}$ and $\overline{q_{t}^{\prime 2}}$. We argued clouds in $3 \mathrm{D}$ to be deeper partly due to reduced drying through lateral entrainment in growing clouds, given the average moister cloudy layer. Due to the larger heterogeneities in 3D and the decoupling from cloud shading below, it is likely that clouds form recurrently over the same regions increasing the moisture locally and thus, grow through a moister environment than the average cloud layer air. This would be further justified by the increased surface flux below clouds due to the clouds reflecting diffuse radiation downwards. Jakub (2016) found longer-lasting clouds with three-dimensional radiation. To reveal preferred location for cloud onset, a brief analysis on accumulated liquid water path over the domain during first hours of convection showed no significant difference between $1 \mathrm{D}$ and 3D radiation, but a more detailed analysis is required. Continuing with the characterization of cloud size, a cloud size density evaluation of the cloud fields would clarify 1) the dominant size of clouds in each case and 2) whether the typical power law-like relation between cloud density and size (Neggers et al., 2003) suffers any modification with three-dimensional radiation. 
Finally, the three-dimensional radiative transfer used here is known to underestimate diffuse light diffusivity and, thus, overestimate the presence of diffuse radiation particularly below clouds. This may lead to exaggerated heterogeneities at the surface and overestimation of circulations or clouds intensification. Possible solutions to reduce the already known too little diffusivity of diffuse radiation would entail increasing the number of streams in the radiative transfer scheme or smoothening the produced surface fluxes or the diffuse radiation at the surface Veerman (2019). 


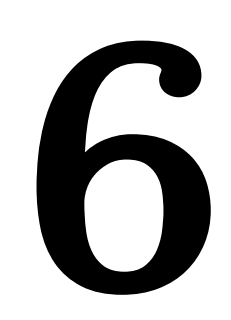

General Conclusions and Outlook 


\subsection{Conclusions}

Within the atmospheric boundary layer the active vegetation and clouds interact within minutes, with radiation conveying the effects of clouds on vegetation. In turn, the vegetation response is conditioned by the radiation and its effect on temperature and atmospheric water demand. As a consequence, the vegetation regulates through photosynthesis the surface partitioning between evapotranspiration and sensible heat flux and, consequently, the turbulent processes in the boundary layer. This thesis investigates these sub-daily vegetation-cloud interactions, and how these interactions can affect both the vegetated surface and the clouds along their development in different boundary-layer conditions. We studied biophysical and turbulent processes at the scales they occur, i.e. meters and seconds, and systematically analyzed how key aspects such as thermodynamics of the boundary layer, dynamic and radiative properties of clouds and surface fluxes and carbon uptake by vegetation vary throughout the day. We simulated the physics of turbulence and cloud convection explicitly using the Large Eddy Simulation (LES) technique, the radiation with state-of-the-art parameterized radiation schemes and the biology of the vegetated surface with a well-tested sub-model. This sub-model consisted of a mechanistic representation of plant photosynthesis and related processes. To cover a wide range of ecosystems and atmospheric conditions, we studied cases located in mid-latitudes such as The Netherlands, and in lower tropical latitudes such as southern West Africa and Brazil (see Figure 1.2 in Chapter 1). To complete this cross-ecosystem and cross-climate study, we analyzed these interactions under different boundary-layer conditions: clear boundary layers and boundary layers topped by shallow cumulus or stratocumulus clouds. We paid special attention to the transition from one boundary layer type to another, and the roles played by clouds and the vegetated surface in effecting such transitions. Below, we summarize the main findings with the corresponding research question as in Section 1.4 .

Are current parameterized calculations of shallow cumulus in weather forecasting models adequate to represent how clouds interact with the surface and the boundary layer? Can we define a research strategy to diagnose the misrepresentations?

In studying the interactions taking place within the atmospheric boundary layer, it is firstly necessary to learn what level of explicitness in the simulation of biophysical processes is required to obtain accurate results. To this end we investigated the ability of mesoscale models representing turbulence and shallow convection with parameterizations to reproduce the dynamic and radiative effects of a shallow cumulus case (Chapter 2). The study has been performed by reproducing a mid-latitude summer day with shallow convection developing in the late morning over homogeneous grassland. Our research strategy was based on performing systematic numerical experiments on this idealized summer day. 
We reproduced the day with two different modeling techniques: turbulence-resolving LES and the most widely employed mesoscale model in which turbulence and shallow convection are represented by state-of-the-art parameterizations. We performed numerical experiments using two different LES models. Given the good agreement between the two LES experiments explicitly solving the turbulent motions we used these LES experiments as reference. We additionally performed four experiments using the mesoscale model with varying horizontal resolutions and representations of shallow convection.

The findings revealed that the mesoscale experiments had difficulties on correctly representing the timing, vertical extent or horizontal coverage of the shallow clouds. Furthermore, cloud-induced dynamics such as moisture ventilation between the subcloud and cloud layer were also misrepresented in the mesoscale experiments. Specifically, the the convective schemes in mesoscale simulations transported too much moisture from the subcloud layer too early and too high into the cloud layer. As a result, the development of successive clouds is affected. Such cloud misrepresentation yielded small alterations in the slab average shortwave radiation at the surface due to a low cloud cover, i.e. of $20 \%$ at its maximum. Despite the little variation on slab average shortwave radiation, the direct and diffuse components of radiation were overestimated and underestimated, respectively. Focusing further on the representation of radiation, we found too strong height-dependent fluctuations of shortwave radiation due to overly abrupt variations in cloud water content with height. We further analyzed the spatial variability of clouds and related radiative effects, and found too strong fluctuations of shortwave radiation. Here, the too large values and spatial variability of liquid water path in clouds explained the overestimation of the spatial variability in shortwave radiation. A refined horizontal resolution from $9 \mathrm{~km}$ to $3 \mathrm{~km}$ without a convective scheme did not provide results comparable to those from LES.

Based on these results we concluded that the current mesoscale models and parameterizations are unable to properly simulate the convection of shallow clouds, their interaction with radiation and the consequent impact at the surface. Thus we concluded that explicit LES simulations of the turbulent flow and cloud dynamics are necessary to adequately resolve and explore the potential interactions between shallow clouds and vegetation. Furthermore, this study showed that schemes used to represent subgrid processes in the mesoscale need to be evaluated simultaneously and interactively, and not as stand-alone parameterization as is currently prevalent. Given the previous conclusions in which we gained a better understanding by explicitly simulating the coupling between clouds and vegetation, we carried on with the LES technique as the main methodology in our thesis.

What is the effect of cloud-driven radiative perturbations, including the direct and diffuse partitioning, on the vegetation responses? Do these surface responses feed back to the boundary layer and clouds? 
Using the LES technique, we explored one of the cloud processes altering the response by active vegetation: the partitioning of shortwave radiation on its direct and diffuse components by clouds (Chapter 3). We also investigated the impact of this partitioning on the active vegetation and, consequently, surface fluxes. To mimic the effects on the vegetated canopy of environmental variables such as surface wind, $\mathrm{CO}_{2}$ concentrations, soil moisture, vapor pressure deficit, shortwave radiation or temperature we employed a coupled land-surface model with a mechanistic representation of the stomatal regulation by plants. We developed and included a simple in-canopy radiative transfer model accounting for sunlit and shaded leaves, i.e. two-big leaves model, where we paid particular attention to modeling the transfer of diffuse radiation: different extinction rates for direct and diffuse radiation were calculated, and the creation of additional diffuse radiation within the canopy due to reflections of light by leaves and the ground was accounted for. The LES study was based on an initially clear sky day in The Netherlands over homogeneous grass with shallow cumulus clouds developing before noon.

We found $\mathrm{CO}_{2}$ assimilation by plants to increase under thin clouds with an optical depth below 10. Consequently, an increase (decrease) in latent heat (sensible) flux due to the plant coupling was found. The larger ratio of diffuse radiation in the cloud-driven partition of shortwave radiation drove the increase in vegetation activity under thin clouds. This was confirmed as an additional experiment where all radiation reaching the canopy was assumed to be direct showed no increased vegetation activity. The total reduction of shortwave radiation was found to dominate over the increase of diffuse radiation under thicker clouds and, consequently, no increased vegetation activity was found there. Similarly, water use efficiency and evaporative fraction rose under increasingly thicker clouds due to the enhanced diffuse radiation. To aid in the interpretation of the LES results, we designed a controlled experiment on a mixed layer model coupled to the land-surface model. We found the stomata opening by vegetation to lead the enhanced carbon uptake under thin clouds. As a consequence of the stomata opening, the carbon gradient between the atmosphere and inside the leaf decreased, partially compensating for the increase in $\mathrm{CO}_{2}$ uptake due to the stomatal opening. The demonstrated distinct impact of clouds on surface fluxes when accounting for diffuse radiation did not, however, feed back to the boundary layer and clouds. The reason lies in the fact that the optimum cloud thickness range enhancing latent heat flux was limited, as well as the thin cloud occurrence. In fact, total cloud cover never exceeded $20 \%$ of the domain.

Thus, although not feeding back to the boundary layer in this case, we concluded that the direct-diffuse radiation partition by clouds augments plant activity under certain conditions, leading to enhanced carbon absorption and latent heat flux. The cloud-driven shift in the surface energy balance may, under other conditions, influence the turbulent and cloudy structure of the boundary layer. Pursuing this line of investigation, Sikma et al. (2018) studied the enhanced vegetation activity under thin clouds over different wind regimes but found a reduced impact by vegetation. These findings exemplify the 
potential of coupling different fields, such as biology and meteorology, to produce features that are absent when studying uncoupled fields.

\section{What are the radiative and dynamic characteristics of a stratocumulus to cumulus transition over land? What is the role played by local physical processes, including the vegetation responses?}

The capacity of vegetation to influence the turbulence and cloud characteristics was further studied in Chapter 4, focusing in this case on stratocumulus clouds and their transition to shallow cumulus. Here, we aimed at covering the gap of knowledge related to the little attention paid by cloud-transitional studies to processes like net radiation, surface fluxes and wind shear at the top of stratocumulus clouds over land. We designed an idealized LES case using the dataset resulting from the intensive observations collected with contribution by the author during the experimental campaign in Save, Benin, as part of the Dynamics-aerosol-cloud Interactions in West Africa (DACCIWA, www.dacciwa.eu) project. Typical conditions observed by the author during the DACCIWA campaign consisted of a homogeneous stratocumulus deck during the night and early morning evolving to broken cumulus clouds in the afternoon. This atmospheric situation was a challenge due to several reasons. Firstly, it had a local character that hypothetically depended on the surface and boundary-layer dynamics, despite the influence by the West African monsoon conditions. Secondly, the air above was as moist as the air within the cloud layer, a very rare feature in the well-studied marine stratocumulus. Lastly, a recurrent low-level jet was observed during the stratocumulus formation and maintenance.

The designed LES case showed an initial nocturnal stratocumulus over land, a subsequent thinning of the deck during the morning and the break up and evolution to a shallow cumulus-topped boundary layer around noon. The interactive land-surface model described above and including the direct-diffuse sensitivity was used to account for surface flux responses to cloud thinning and dynamic heterogeneities at the surface. This is to our knowledge, the first LES study on the stratocumulus to cumulus transition over land strongly constrained by observations and evaluated against a set of independent measurements. We performed a thorough characterization of the transition in terms of boundary layer dynamics, radiation and cloud properties. We further quantified the physical processes relevant for the stratocumulus cloud thinning and subsequent breakup of the deck. Longwave cooling at cloud top lead a net thickening of the cloud layer during the first hours. After sunrise the shortwave radiation, affecting net radiation both at cloud top and surface, drove the thinning through a sum of processes: it weakened the cooling at cloud top and increased the entrainment of warm and drier air due to the additional increase of surface fluxes. The presence of wind shear accelerated the breakup by 2 hours by hampering the nocturnal thickening of the cloud layer and enhancing entrainment after sunrise. Finally, and to describe metrics used by larger scale models to parameterize the prototypical clear and cloudy boundary layers, we quantified the ratios of subcloud to 
surface buoyancy and moisture fluxes. The former showed a linear decrease during the transition reaching typical values of the convective boundary layer in the afternoon. Wind shear was found to accelerate such transition by about 2 hours. The latter grew from 0.8 to values above 1 during the cloud transition, indicating a shift from net moistening to a net drying of the subcloud layer.

These results showed explicitly the evolution of radiative and dynamic properties of the boundary layer and clouds from the homogeneous nocturnal stratocumulus conditions to the shallow cumulus-topped boundary layer in the afternoon. Furthermore, results demonstrate that the interplay between radiation and the surface plays a critical role in the thinning of the stratocumulus layer and its subsequent breakup, with wind shear accelerating the transition.

\section{What are the differences between using one-dimensional radiation compared to a more realistic three-dimensional radiation on a shallow cumulus-topped boundary layer with active vegetation? Do the surface, boundary layer and cloud properties change?}

An important conclusion from the previous paragraphs is the key role played by radiation in the vegetation-clouds interaction. In other words, the net reduction of shortwave radiation and the shift of direct and diffuse radiation ratio by clouds play a key role in the photosynthesis cycle, evapotransportation and generated turbulent intensity at the surface. The common approach in three-dimensional LES studies (including our previous results) to simulate the radiative transfer in the boundary layer is to use a one-dimensional radiative transfer model, thus constraining the possible direction of light to the vertical dimension. Yet the magnitude of solar intensity follows the diurnal cycle dependent on the latitude and longitude. The reason for the simplification to one dimension in radiative transfer solvers is the high computational costs of using three-dimensional solvers at the frequency required by LES experiments, i.e. in the order of minutes. The simplification made using a one-directional radiative transfer is particularly relevant when simulating situations with partial cloudiness, because of the neglected shade tilting and cloud-side reflection of radiation. Shade-tilting may decouple the surface at which the cloud is rooted from its shading, while cloud-side reflections may locally enhance radiation above values of clear sky conditions. To move beyond the one-dimensional simplification, in Chapter 5 we employed an innovative and computationally efficient three-dimensional radiative transfer model and compared it to the common one-dimensional approach. For the sake of consistency with the research presented in the previous chapters, we used the same interactive land-surface model. This enabled us to investigate whether the distinct radiative patterns in the three-dimensional case yielded differences in carbon uptake and surface fluxes, with potential implications on turbulent and cloud structures within the boundary layer. We performed the numerical experiments based on a shallow cumulus day over the Amazonian rainforest. The experiment reproduced a characteristic situation 
of the dry season in September that may be relevant for the onset of the wet season during the month of October.

We found that the $3 \mathrm{D}$ radiation scheme was able to calculate a wider and more complete range of values and combinations of direct and diffuse radiation at the surface. This allowed new an realistic features to appear, such as locally augmented carbon absorption and, thus, enhanced surface fluxes, due to lateral reflections of neighboring clouds. The upper half of the subcloud layer showed slightly larger turbulent and moisture transport in the 3D case during the first hours after cloud onset. Consequently, these yielded larger liquid water in the cloud layer. After the shallow cumulus reached their maximum cover (13 00 Local Time) the solar zenith angles was large enough to exert significant differences between the 1D and 3D experiments: more intense updrafts developed in the 3D case, with the subsequent increased transport of moisture to the cloud layer. As a consequence, the size of clouds increased to almost twofold the average cloud size in the $1 \mathrm{D}$ case, from 300 to $500 \mathrm{~m}$ across-cloud distance. This was corroborated by clouds in 3D showing larger vertical velocities and transport of moisture, particularly after 13 LT.

These results reveal the impact of processes usually neglected in one-dimensional LES experiments and indicate the potential of three-dimensional radiation coupled to the surface to modify the turbulence and cloud features. Furthermore, it sets the path for further investigations on the consequences of solving the three-dimensional radiative transfer and the direct and diffuse partitioning on shortwave radiation.

\subsection{Outlook}

This thesis investigates how the small spatiotemporal scales influence the couplings between vegetation and clouds within the atmospheric boundary layer. It presents innovative studies on the interactions and consequences of simulating cloudy boundary layers with a coupled vegetation-mimicking sub-model (see Fig. 1.1 in Chapter 1). Although clouds, active vegetation, radiation and turbulence have been studied in great detail in their respective fields, this is a first attempt to integrate systematically the biophysical processes and quantify their interactions resolving the main physical equations as explicit as possible. As such, the research carried out in this thesis is only a first step towards the understanding of the relation between vegetation, clouds, radiation and turbulence within the boundary layer as an integrated system and towards the evaluation of the interacting modeled representations. Here we provide a set of recommendations paving the way for future research in the topic of clouds and vegetation interactions at small spatiotemporal scales. 


\subsubsection{Boundary-layer clouds}

Comparing explicit LES experiments with experiments on larger scale models with parameterized turbulence and cloud dynamics, we found clear evidence of the misrepresentation of basic shallow-convection features in Chapter 2. Two of the main misrepresentations found were the overestimated transport of moisture by clouds soon after cloud onset, and the incorrect partitioning of direct and diffuse radiation due to clouds. We attributed the overly large moisture transport to an underestimation of lateral entrainment into clouds and updrafts by convective parameterizations. Correcting this misrepresentation, however, would require further verification and study, and a consequent refinement of the representation of such processes to gain realism. Similarly, a revision on the information exchanged between convective and radiative parametrization would improve the direct-diffuse estimates based on the properties of parameterized clouds.

We focused here on the effects exerted by vegetation on clouds mostly through the surface fluxes and subsequent turbulent effects. Other processes belonging to the field of atmospheric chemistry, such as the hypothetical effects of isoprene emissions from vegetation to act as precursors of cloud condensation nuclei via the formation of secondary organic aerosols, have been neglected but deserve further exploration. This leads us to one of the unexplored processes in this thesis, with the potential to alter clouds: their microphysical properties. We studied the macrophysical and dynamic properties but did not carry out an in-depth study into how the representation of microphysics with different degrees of complexity might alter cloud dynamics, radiation and the subsequent interaction with vegetation. Whether these processes, when considering vegetation aerosol-precursor emissions, alter cloud cover or other characteristics remains still unexplored.

The three-dimensional radiative transfer developed by Jakub and Mayer (2015) and used in Chapter 5 opens a wide range of possibilities in the research of cloud-surface interactions. Our findings suggest that new features arise when accounting for the threedimensional character of light, such as larger and more buoyant clouds. This suggests that previous cases with a one-dimensional radiative transfer and an interactive landsurface model showing a good match to observations may be obtaining the right results for the wrong reasons. The differences compared to the traditionally used onedimensional schemes demand a revisit of significant LES studies where heterogeneous clouds are present over land. This includes most convective cases over land where the surface plays an important role, but also stratocumulus cases over land with breakups of the cloud deck as the one described in Chapter 4. Following on the possibilities of three-dimensional radiation coupled to the surface, further work is necessary to study its effect on clouds: cloud size distributions, spatial organization patterns and cloud dynamics should be revised. Furthermore, the 3D effects of radiation may have larger impacts at higher latitudes due to the large solar zenith angle. A potential study target would be ecosystems such as boreal forests. 


\subsubsection{The vegetated canopy}

The vegetation sub-model used in this thesis to represent the photosynthesis and the exchange of water and $\mathrm{CO}_{2}$, i.e. A-gs, was developed by Jacobs (1994). This model was built based on observations of grapevine plants, i.e. Vitis Vinifera and assumes that vegetation in general follows the same principles. The common approach when designing a case for LES simulation is to obtain slab average surface fluxes matching the observed ones using as many observations of the soil and the canopy as available. However, the variations of surface fluxes and their response intensity to environmental variations may still not be adequately represented by the plant submodel, as different kinds of vegetation may show different response rates or sensitivities to environment modifications. This problem suggests a future avenue for research: to carry out extensive and detailed measurements on the behavior and vegetation characteristics needed by the A-gs model for various plant types and under different meteorological conditions, further improving the validity of such model. A starting point could be to fully characterize vegetation and soil conditions in meteorological observational campaigns to constrain the design of cases in models and further contribute to the correct validation of land-surface models.

The mentioned distinct effect of direct and diffuse radiation revealed enhanced carbon uptake above clear sky values under optimal conditions. Our results, based on one single case, would benefit from similar studies under different cloud types and vegetation conditions, to explore the universality of such feature and its actual relevance in longer term or global estimates. In any case, incorporating such features, i.e. direct-diffuse sensitivities in the canopy, to modeled vegetation by climate and earth system models will lead to more accurate predictions of the global carbon sink and its sensitivity to cloud and aerosol concentration variation.

The effects of radiation on vegetation were taken into account in this thesis through a mechanistic plant submodel, where the representation of the stomatal aperture and photosynthesis depended on the amount of direct and diffuse radiation given their distinct penetration rates in the canopy. The in-canopy radiative transfer scheme used here presents a simplified simulation of light penetration where vegetation is seen as a uniform mesh, and assumes a horizontally homogeneous canopy. A more realistic representation of the radiative transfer within the canopy would provide more reliable responses of vegetation to radiation perturbations. Here, we suggest new approaches for simulating the radiative transfer within the canopy with higher accuracy. An example of this is the work by Kobayashi et al. (2012), where they use a three-dimensional radiative transfer within the canopy, where, among other features, the canopy is distinguished from the stem of the vegetation and photons are randomly sent from above the canopy to calculate the different possible directions and path lengths within the canopy. Such canopy radiative transfer was found to better represent the canopy understory properties and the spatial heterogeneities within the canopy otherwise absent in one-dimensional schemes. This approach 
would reinforce the three-dimensional character of light by a coupling to an atmospheric three-dimensional radiation transfer scheme as the one used here and developed by Jakub and Mayer (2015), allowing for a full three-dimensional radiation approach above and inside the canopy. We realize that the computational costs are very high. Yet we believe that these academic experiments can contribute to asses the validity of current parameterizations, design new ones and help in the convergence between increasingly detailed observations and explicit numerical simulations.

In this thesis we mainly focused on the effects that clouds exert on vegetation due to radiation perturbation. However, clouds modify many other aspects affecting vegetation, such as the surface temperature and related vapor pressure deficit, or near surface wind. Thus, further research in the topic should move in the direction of quantifying each of these processes to better understand the short term impact of clouds on vegetation. For this purpose it would be beneficial to explicitly simulate the flow within the canopy and to allow for different thermodynamic conditions along the canopy height, i.e. multi-layer in canopy model, as already explored by Finnigan et al. (2009) and Ouwersloot et al. (2016). This improved description of leaf and canopy should be accompanied by a revision of the validity of the relation between the surface turbulent fluxes and the atmospheric gradients above the canopy. In our work, we have assumed that surface fluxes and state variable gradients are related using the formulation based on Monin-Obhukov Similarity Theory, a framework assumption that may not hold under the unsteady and spatially heterogeneous conditions studied here.

On the observational side, information of short-term interactions between the vegetation and cloud perturbations is needed. In this sense, work by Kivalov and Fitzjarrald (2018) and Kivalov and Fitzjarrald (2019) is an example of how surface observations can contribute to the understanding and validation of the modeled interactions and the response of vegetation. In the mentioned studies, cloud-driven fast fluctuations in surface radiation were measured, classified and interpreted, as well as their impact on thermodynamic conditions in the canopy and, consequently, on surface fluxes.

\subsubsection{Vegetation-cloud interactions}

Under a changing climate in which values of key variables such as temperature and $\mathrm{CO}_{2}$ are increasing, it is uncertain whether the interactions found in this thesis still hold. The current warming and high-levels of $\mathrm{CO}_{2}$ are modifying vegetation behavior as well as the atmosphere's thermodynamic state. Studies investigating future climate scenarios, e.g. with increased $\mathrm{CO}_{2}$ concentrations and temperatures, are needed. One such study by Sikma et al. (2019) looked at the changes in the cloud-vegetation coupling and its impact on boundary layer on a simplified future climate with warmer and $\mathrm{CO}_{2}$ richer atmosphere. However, only the physiological $\mathrm{CO}_{2}$ forcing was studied, i.e. the effect of enhanced $\mathrm{CO}_{2}$ 
in vegetation through the stomata closure due to the $\mathrm{CO}_{2}$ fertilization effects, whereas the known atmospheric $\mathrm{CO}_{2}$ forcing, i.e. the fact that $\mathrm{CO}_{2}$ molecules modify shortwave and, specially, longwave radiation, is neglected. Studies accounting for both processes are necessary, given the potential of radiative $\mathrm{CO}_{2}$ forcing to alter cloud dynamics (Schneider et al., 2019).

Finally, the interactions studied in this thesis should be tested in less idealized conditions. In most of our studies we assumed in windless conditions with immediate surface response to forcings and no large scale dynamics. Adding any of these effects will make the experiments more realistic and help test whether the findings described here are still of relevance when other atmospheric phenomena appear. The study by Sikma et al. (2018) is an example of an addition of these effects, where they studied the shallow cumulus-vegetation coupling under several wind conditions and lag on the vegetation response.

We also advice the use of a non-dimensional approach for further work both in the vegetation-cloud interactions and boundary-layer studies in general. Numerous studies have presented results on boundary layer evolutions with varying results in apparently similar problems. In a majority of cases, the results differ due to the relative difference between the magnitude of certain processes, with the conclusions being drawn on the absolute values of the processes. The use of non-dimensional parameters, where relative importance of the ruling processes in comparison to others is emphasized, may be a tool to provide more consistent results regardless of the particular case and conditions. van Heerwaarden et al. (2014) provides an example of this approach to study the role of surface heterogeneities in the convective boundary layer.

In summary, our aim in this thesis was to provide quantitative insights into the subdaily interactions between the vegetated surface and boundary-layer clouds as part of the boundary layer evolution. We performed systematic numerical experiments to reveal the role played by the vegetated surface and described the boundary layer evolution. We also encountered several limitations: in studying short spatiotemporal scales we found that critical processes, such as radiation and canopy responses, need to be represented in more detail. Thus, any future steps towards a more realistic, and computationally affordable, representation of such processes will further contribute to the original goal of this thesis: to understand, describe and quantify the interactions between the vegetated surface and the clouds in the sub-daily scale as part of the boundary layer. 



\section{Summary}

This thesis deals with the interactions between the boundary-layer clouds and the active vegetation. The diurnal evolution of the atmospheric boundary layer is conditioned by its lower boundary, i.e. the vegetated surface, and the presence of boundary layer clouds. The clouds can show vertical development such as the cumulus clouds, or be horizontally homogeneous such as the stratocumulus. The photosynthesis and the related stomatal aperture determines the $\mathrm{CO}_{2}$ and water exchange between the plants and the atmosphere. As a result, the vegetation activity regulates the water vapor entering the atmosphere at surface and, consequently, the partitioning of available energy at the surface into latent and sensible heat fluxes, the so-called surface energy balance. These surface fluxes drive the atmospheric turbulence, that is the main process transporting heat and moisture within the boundary layer. Furthermore, heat and moisture are the key variables in the formation and intensification of boundary-layer clouds. The system is more complex since boundary-layer clouds reduce the available radiation at the surface, and therefore modify the conditions in which photosynthesis and stomatal aperture are acting. In cases with meteorological situations characterized by partial cloudiness, such as shallow cumulus or broken stratocumulus, clouds introduce dynamic heterogeneities at the surface by shading surface patches. Such dynamic heterogeneities lead to different vegetation responses depending on the available radiation and, thus, varying surface fluxes. The main aim of this thesis is to unravel the bi-directional effects between vegetation and clouds and to quantify the main processes. We investigate this cloud-vegetation system over different ecosystems and cloud field characteristics.

Our main method consists of numeric simulations based on the solution of physical laws in which we integrate mechanistic representations of photosynthesis and plant stomatal aperture. To perform the experiments we make use of idealized simulations using the Large Eddy Simulation (LES) technique. In LES the turbulent motions of the flow within the boundary layer are numerically explicitly resolved. This includes the cloud dynamics. We couple the LES model to a land-surface model with a mechanistic representation of vegetation mimicking the stomatal aperture and closure in leaves. In this submodel the photosynthesis rates depend on environmental variables such as $\mathrm{CO}_{2}$ concentration, air temperature and specific humidity, soil moisture, near surface wind and shortwave radiation. In this thesis special attention is paid to develop the submodel by extending it to be a two-big leaf scheme. Such scheme simulates the canopy photosynthesis by sunlit and shaded leaves as well as different one-dimensional in-canopy penetration efficiencies for direct and diffuse radiation. We further include the diffuse radiation created in the canopy by reflections of direct radiation by leaves or by the ground. All the LES experiments in this thesis are performed with one-dimensional radiative schemes, except for Chapter 5 . There, we investigate the effects that a three-dimensional radiative scheme coupled to the surface exerts on a shallow cumulus case. Three dimensional-radiative transfer solvers 
allow for realistic features neglected in one-dimensional schemes, such as the tilting of cloud shades or the reflection of light by the lateral parts of clouds.

In Chapter 2 we study whether the current mesoscale models and parameterizations are able to properly describe the dynamic and radiative impact of shallow cumulus and the subsequent response by vegetation. Mesoscale models do not explicitly resolve the turbulent motions in the boundary layer and clouds. Instead, they use computationally faster representations that depend on parametric expressions to consider the effects of such motions at the spatial scales simulated. These representations are known as parameterizations. We compare mesoscale simulations with varying horizontal resolutions and parameterizations for shallow convection against two explicit LES numerical experiments by different models. The case under study is a late-summer day in mid-latitudes, with clear sky in the morning and shallow clouds developing around noon. The settings of the mesoscale simulations are typical of numerical weather forecasting models. We find that the simulations parameterizing clouds and turbulence are unable to describe the dynamic and radiative effects of shallow cumulus clouds when compared to the coinciding LES simulations. They predict a too early onset of clouds and overestimate the transport of moisture by the clouds. These leads to the creation of a second cloud layer too high. The cloud cover and horizontal cloud fraction are lower than in LES experiments, suggesting that the too active few clouds inhibit the creation of more and smaller clouds. Cloud fraction also shows a lack of dynamism in its evolution by exhibiting nearly constant values after 13 Local Time (LT). The thermodynamic consequences of this misrepresentation is a too warm (up to $2 \mathrm{~K}$ warmer) and too dry (up to $2 \mathrm{~g} \mathrm{~kg}^{-1}$ drier) cloud layer with moisture accumulation higher up. The misrepresentation of clouds has further effects on the radiative fields. Shortwave radiation is abruptly reduced along the vertical direction due to too strong variations of cloud liquid water content with height. Similarly, the horizontal variability and magnitude of liquid water path is overestimated in mesoscale simulations. This leads to an overestimation of shortwave radiation variability at surface. We further study the partitioning of shortwave radiation into its direct and diffuse components, and find that the mesoscale simulations largely underestimate the cloud-induced diffuse radiation increase by as much as 50\%. Although not studied in this chapter, this misrepresentation of the direct-diffuse partitioning may influence regional $\mathrm{CO}_{2}$ estimations and cloud forecast.

The remaining part of the thesis deals with experiments performed using LES, since Chapter 2 showed that parameterizations are currently not able to describe properly the radiative and dynamic effects of shallow cumulus clouds. The radiative effects of shallow cumulus clouds on the active vegetation is studied in more detail in Chapter 3. In particular, we focus on the surface turbulent fluxes of $\mathrm{CO}_{2}$, water and heat. In that chapter we design an idealized numerical experiment on LES inspired by typical summer conditions in The Netherlands over homogeneous grass. We pay special attention to the partitioning of shortwave radiation into direct and diffuse components, and how this modifies the veg- 
etation photosynthesis. Diffuse radiation is known to enhance photosynthesis at canopy scale due to a more homogeneous spread of light within the canopy. We further investigate whether such partitioning leads to differences in boundary-layer properties and cloud dynamics. Results show that considering the partitioning of shortwave radiation into its direct and diffuse components leads to new features in the vegetation response: under thin clouds, defined as clouds with a cloud optical depth $\tau<10$, the effect of diffuse radiation on vegetation is stronger than the total reduction of radiation in the canopy. As a consequence, we find larger $\mathrm{CO}_{2}$ assimilation, up to $20 \%$, under thin clouds than under clear sky. Since photosynthesis couples the $\mathrm{CO}_{2}$ assimilation and the water vapor released by vegetation, we also find enhanced (decreased) latent (sensible) heat flux under thin clouds. This shift in surface fluxes leads to enhanced water use efficiency under thin clouds. These effects disappear under thicker clouds or when the direct-diffuse partition of shortwave radiation is neglected. To support our data interpretation we set up a controlled experiment in a mixed-layer model coupled to the same land-surface model to quantify the processes leading to the photosynthesis enhancement under thin clouds. By prescribing the onset of a thin cloud and observing the response of the active vegetation, we find the following sequence: an opening of the stomata due to enhanced diffuse radiation leads to increased carbon assimilation. As a response, the carbon gradient between inside and outside the leaf decreases, also aided by a cooling of the shaded surface. This gradient decrease has a negative contribution to the carbon assimilation. The carbon assimilation positive tendency is further decreased by a slight reduction in the aerodynamic resistance due to colder surface and, consequently, lower convective velocities near surface. Overall the stomatal aperture effect is dominant and, therefore, the net effect of the thin-cloud shading is an increase in carbon assimilation by the plants.

Since the direct-diffuse radiation partitioning leads to a shift of surface fluxes below clouds, we further investigate whether these modifications at surface have an impact on the properties of the boundary layer and the shallow clouds. We find that the modifications in surface fluxes are not significant enough to feed back to the boundary layer due to two reasons. Firstly, the enhancement (decrease) of latent (sensible) heat flux is relatively small, by up to $10 \%$ for latent heat flux. Furthermore, the range of cloud thicknesses required for these effects to happen is very limited, and such clouds are not frequent enough during the numerical experiments. In fact, cloud cover never exceeds $20 \%$ in the experiments.

We further investigate the cloud-vegetation influences in a different climate and boundary layer in Chapter 4. There, a new LES case is designed based on the extensive observational dataset gathered during the experimental campaign of the Dynamics-Aerosol-ChemistryCloud Interactions in West Africa (DACCIWA) project with the contribution of the author. The campaign collected intensive observations in Benin, southern West Africa, on the thermodynamic state of the boundary layer, boundary-layer clouds and surface conditions. The designed LES case mimics a frequent succession of events observed during the 
campaign: an initially low and homogeneous stratocumulus cloud deck forming over land during the night and early morning, showing a gradual lifting of the cloud base after sunrise. Afterwards, the cloud deck thins and breaks up, leading to dynamic heterogeneities at surface. After noon, the cloud layer transitions to shallow cumulus clouds. Multiple processes are acting during this transition happening within few hours. Based on the designed LES case we describe and quantify the transition from nighttime stratocumulus to shallow cumulus clouds in the afternoon. We quantify the transition in terms of thermodynamic, radiative and turbulent vertical profiles. In addition, we describe the decoupling between the cloud layer and the surface, the evolution and widening of the cloud layer and inversion layer as well as dynamically relevant heights such as the height of minimum buoyancy flux or subcloud layer top.

To gain a deeper understanding on the transition, we perform a liquid water path budget analysis to reveal the physical processes leading to the thinning and subsequent breakup of the stratocumulus deck. The longwave radiative cooling at the cloud top dominates the thickening of the cloud layer before sunrise with rates of more than $40 \mathrm{~g} \mathrm{~m}^{-2} \mathrm{~h}^{-1}$. Subsidence shows a nearly constant negative contribution of about $20 \mathrm{~g} \mathrm{~m}^{-2} \mathrm{~h}^{-1}$ during the whole experiment. During the night there is little contribution by other processes. As a result the overall effect is a net thickening of $20 \mathrm{~g} \mathrm{~m}^{-2} \mathrm{~h}^{-1}$, equivalent to a relative $15 \%$ growth per hour. After sunrise the shortwave radiation affects the budget in two ways: it weakens the cooling by longwave radiation and increases the surface fluxes, leading to enhanced entrainment. Both processes lead to a thinning of the cloud layer starting about 75 minutes after sunrise. Additional LES experiments including wind profiles representing main features of the observed low-level jet show that wind shear at cloud top hampers the thickening of the cloud layer during the night and accelerates the thinning and breakup of the deck through two effects: the direct dynamical effect of wind shear at cloud top increasing entrainment, and the increase of surface fluxes after sunrise due to a thinner cloud layer. Similarly, wind shear widens the cloud layer and the inversion layer and accelerates the decoupling of the cloud layer. We describe the time evolution of subcloudtop-to-surface flux ratios for both buoyancy and moisture. These ratios are frequently used by larger-scale model parameterizations to represent the boundary-layer dynamics. The ratio of buoyancy shows a linear decrease starting after sunrise and plateau-ing at values of -0.2 , typical of convective boundary layers, at about 12:30 LT. The moisture ratio decreases from 0.8 to 0.7 before cloud breakup showing a net moistening of the cloud layer and grows steadily after the breakup showing a net drying of the subcloud layer. Wind shear is found to accelerate the tendencies of the buoyancy and moisture ratios.

Chapter 5 aims at studying the impact of the radiative transfer perturbed by clouds. We analyze and quantify the implications of using a one-dimensional (1D) radiative transfer model compared to an innovative three-dimensional (3D) radiative scheme when coupled to a land-surface model. This is one of the first attempts to simulate a shallow cumulus 
case with active vegetation coupled to a $3 \mathrm{D}$ radiative scheme. These radiative schemes are usually computationally very expensive. The employed 3D scheme has been recently developed and solves relatively fast the radiative transfer allowing for 10 directions for diffuse radiation and 3 for direct radiation, instead of the 2 directions allowed, upwards and downwards, in common 1D schemes. We perform LES simulations with the 1D and the more realistic and computationally demanding 3D radiative schemes on a shallow cumulus case over the Amazonian rainforest in Chapter 5. We analyse the radiative effects at surface and the vegetation response, as well as the differences in boundary layer turbulence and cloud dynamics and structure. Results show that the 3D radiation yields observed features such as local radiation values at surface higher than under clear sky due to lateral reflections of clouds. We find that the reflected radiation may locally add up to $60 \%$ of the radiation received during the hour of highest irradiation, i.e. between 11:30 and 12:30 LT. This leads to local enhancements of similar magnitude on carbon assimilation and latent heat flux by vegetation. In addition, 3D shows a wider range of values for direct and diffuse radiation at the surface, and subsequently, a wider range of latent heat flux values. The 3D experiment shows larger turbulence kinetic energy and updrafts in the upper half of the subcloud layer after 13 LT. This coincides with stronger turbulent fluxes of buoyancy and moisture within the cloud layer. We attribute the increase to the tilting of the cloud shade that decouples the shade from the source of the updraft on which the cloud is rooted. The increase in cloud activity leads to a more moist cloud layer. The effects of 3D radiation lead to simulations characterized by wider clouds after 13 LT. While in $1 \mathrm{D}$ the cross-size of clouds stabilizes at $300 \mathrm{~m}$, in 3D the average cloud size grows up to $500 \mathrm{~m}$ due to the shade tilting. This increase in size is reinforced by stronger upward motions and transport of moisture inside clouds.

This thesis presents pioneering investigations on the interactions of boundary-layer clouds and the active vegetation within the atmospheric boundary layer. It integrates knowledge from different fields into one system and addresses issues related to the potential reactions that clouds and plants have to each other through their effects on radiation and turbulence. We design LES experiments to systematically disentangle the numerous interactions and provide the first insights of the relations found. Results show that the onset, maintenance and dissipation of boundary-layer clouds are closely linked to the vegetation activity and the plant responses to cloud shading. In turn, we conclude that photosynthesis-related processes such as stomatal aperture are also strongly modified by the cloud dynamics. We furthermore provide suggestions of further work with the intention to progress on the understanding of the vegetation-cloud interactions in the boundary layer as a coupled system. 



\section{Resumen}

Esta tesis trata sobre las interacciones entre las nubes en la capa límite y la vegetación activa. La evolución diurna de la capa límite atmosférica está condicionada por su límite inferior, i.e. la superficie con vegetación, y la presencia de nubes en la parte superior. Las nubes pueden mostrar desarrollo vertical como en el caso de las shallow cumulus o cúmulos someros, o ser horizontalmente homogéneas como los estratocúmulos. La fotosíntesis y la apertura de estomas en las plantas determina el intercambio de agua y $\mathrm{CO}_{2}$ entre la vegetación y la atmósfera. Por tanto, la actividad vegetal regula el vapor de agua entrante desde la superficie a la atmósfera y, como consecuencia, la partición de la energía disponible en la superficie en flujos de calor sensible y latente. Esta partición es conocida como balance de energía superficial. Los flujos de superficie controlan en parte la turbulencia atmosférica, el principal proceso de transporte de calor y humedad dentro de la capa límite. Además, la temperatura y la humedad son variables clave en la formación e intensificación de las nubes en la capa límite. El sistema es más complejo ya que estas nubes reducen la radiación disponible en la superficie, modificando así las condiciones bajo las que actúan la fotosíntesis y apertura de estomas. En situaciones meteorológicas caracterizadas por nubosidad parcial, como cúmulos someros o estratocúmulos rotos, las nubes añaden heterogeneidades dinámicas en la superficie mediante las zonas sombreadas. Estas heterogeneidades dinámicas provocan diferentes respuestas en la vegetación dependiendo de la radiación disponible y, por tanto, cambios en los flujos de superficie. El objetivo principal de estas tesis es descifrar los efectos bidireccionales entre la vegetación y las nubes y cuantificar los principales procesos. Se investiga este sistema nube-vegetación sobre diferentes ecosistemas y situaciones nubosas.

Nuestro método principal consiste en simulaciones numéricas basadas en la solución de las leyes físicas donde integramos una representación mecanística de la fotosíntesis y la apertura de estomas. Para llevar a cabo los experimentos utilizamos simulaciones idealizadas mediante la técnica de Large Eddy Simulation (LES) o simulación de grandes eddies. En LES los movimientos turbulentos del aire en la capa límite son resueltos numéricamente de forma explícita. Esto incluye la dinámica dentro de las nubes. Acoplamos el modelo LES a un modelo de superficie terrestre con una representación mecanística que imita la apertura y cierre estomatal en las hojas. En este sub-modelo la fotosíntesis depende de variables medioambientales como la concentración de $\mathrm{CO}_{2}$, la temperatura del aire, humedad específica, humedad del suelo, radiación de onda corta y viento cerca de la superficie. Esta tesis presta especial atención al desarollo del sub-modelo al extenderlo a un two-big leaf scheme o esquema de dos hojas grandes. Este esquema simula la fotosíntesis del dosel arbóreo o canopy mediante las hojas soleadas y sombreadas, además de tener diferentes grados de penetración unidiminesional para la radiación directa y difusa. Incluimos además la radiación difusa creada en el dosel por los reflejos de radiación directa en las hojas y en el suelo. Todos los experimentos LES de esta tesis se han realizado con 
esquemas de radiación unidiminensionales excepto los del Capítulo 5 o Chapter 5 . En ese capítulo investigamos los efectos causados por un esquema de radiación tridimensional acoplado a la superficie en un caso de cúmulos someros. El esquema radiativo tridimensional permite la aparición de efectos reales ausentes en esquemas unidimensionales, como la inclinación de la sombra de las nubes o los reflejos de luz en los laterales de las nubes.

En el capítulo 2 estudiamos la capacidad de los actuales modelos de mesoescala y sus parametrizaciones para describir adecuadamente el impacto dinámico y radiativo de los cúmulos someros y la consecuente respuesta de la vegetación. Los modelos de mesoescala no resuelven explícitamente los movimientos turbulentos en la capa límite y en las nubes. En su lugar, emplean representaciones computacionalmente más rápidas y dependientes de expresiones paramétricas para cuantificar los efectos de los movimientos turbulentos en las escalas espaciales que se simulan. Estas representaciones se conocen como parametrizaciones. En el Capítulo 2 se comparan simulaciones de mesoescala con diferentes resoluciones horizontales y parametrizaciones de cúmulos someros con dos experimentos numéricos realizados mediante modelos LES diferentes. El caso estudiado es un día de fin de verano en latitudes medias, con el cielo inicialmente despejado durante la mañana y el desarrollo de cúmulos someros hacia el mediodía. La configuración de las simulaciones de mesoescala es la típica de modelos numéricos de predicción meteorológica. Se observa la incapacidad de las simulaciones con nubes y turbulencia parametrizadas para describir los efectos dinámicos y radiativos de los cúmulos someros comparado con las simulaciones LES coincidentes. Las simulaciones de mesoescala predicen una aparición demasiado temprana de nubes y sobreestiman el transporte de humedad a través de las nubes. Estos efectos conllevan la creación de una segunda capa de nubes demasiado alta. La cobertura de nubes y fracción nubosa horizontal son menores que en los experimentos LES. Esto sugiere que las pocas nubes existentes son demasiado activas y limitan la aparición de más nubes menores. Los perfiles verticales de cobertura nubosa indican una evolución falta de dinamismo con valores prácticamente constantes después de las 13 hora local (HL). Las consecuencias termodinámicas de esta distorsión son una capa de nubes demasiado caliente (hasta $2 \mathrm{~K}$ ) y seca (hasta $2 \mathrm{~g} \mathrm{~kg}^{-1}$ más seca) mostrando una acumulación de humedad a mayor altura. Esta mala representación conlleva efectos adicionales en los campos radiativos. Las variaciones en altura demasiado abruptas de agua líquida en nube reducen de manera demasiado drástica la radiación de onda corta en dirección vertical. Asimismo, las simulaciones de mesoescala sobreestiman la variabilidad horizontal y magnitud de la columna integrada de agua líquida (liquid water path). Esto explica la subestimación en la variabilidad de onda corta en la superficie. Se analiza, además, la división de onda corta en sus componentes directo y difuso. Encontramos que las simulaciones de mesoescala subestiman en gran parte el incremento de radiación difusa creado por las nubes en hasta el 50\%. Aunque no ha sido estudiado en este capítulo, estas desviaciones en la representación de los componentes directo y difuso de la radiación 
de onda corta pueden influir en las estimaciones regionales de $\mathrm{CO}_{2}$ y la predicción de nubes.

La parte restante de esta tesis muestra experimentos realizados mediante LES para responder a las preguntas propuestas, ya que en el Capítulo 2 se demuestra que las parametrizaciones actuales no describen correctamente los efectos dinámico-radiativos de los cúmulos someros. Los efectos radiativos de las cúmulos someros en la vegetación se estudian con más detalle en el Capítulo 3. En particular, el estudio se centra en los flujos de superficie turbulentos de vapor de $\mathrm{CO}_{2}$, agua y calor. En este capítulo se diseña un experimento numérico idealizado en LES inspirado en situaciones típicas de fin de verano en Holanda sobre hierba homogénea. Se presta especial atención a la división de la radiación de onda corta en sus componentes directo y difuso, y cómo ésta modifica la fotosíntesis de la vegetación. La radiación difusa es conocida por aumentar la fotosíntesis a escala del dosel arbóreo (canopy) debido a una distribución más homogénea de la luz a lo largo del dosel en comparación con la de la radiación directa. Se investiga, además, cómo esta división produce diferencias en las propiedades de la capa límite y en la dinámica de nubes. Los resultados muestran que al incluir la división de la radiación de onda corta en sus componentes directo y difuso aparecen nuevas características en la respuesta de la vegetación: bajo nubes delgadas, definidas como aquéllas con espesor óptico de nubes (cloud optical depth) $\tau<10$, el efecto de la radiación difusa es mayor que el de la reducción de radiación total en el dosel. Esto resulta en una mayor asimilación de $\mathrm{CO}_{2}$ de hasta el $20 \%$ bajo nubes delgadas comparado con valores bajo cielo despejado. Debido al acoplamiento entre vapor de agua emitido por la vegetación y asimilación de $\mathrm{CO}_{2}$ mediante la fotosíntesis, se encuentra un incremento (disminución) de flujo de calor latente (sensible) bajo nubes delgadas. Este cambio en los flujos de superficie causa una mayor eficiencia de uso de agua (water use efficiency) bajo nubes finas. Estos efectos desaparecen bajo nubes más gruesas o cuando se obvia la división de la radiación de onda corta en sus componentes directo y difuso. Para reforzar la interpretación de los resultados diseñamos un experimento controlado en un modelo de capa mezclada (mixed-layer model) acoplado a un modelo de superficie terrestre idéntico al de LES. Así, se cuantifican los procesos que llevan al aumento de fotosíntesis bajo nubes delgadas. Prescribiendo la aparición de una nube delgada y observando la respuesta de la vegetación activa, se encuentra la siguiente secuencia: la apertura de las estomas debido a la mayor radiación difusa aumenta la asimilación de $\mathrm{CO}_{2}$. En respuesta a este proceso, el gradiente de carbono entre el interior y exterior de la hoja decrece, reforzado además por el enfriamiento de la superficie sombreada. Esta reducción del gradiente tiene una contribución negativa en la asimilación de $\mathrm{CO}_{2}$. La tendencia positiva de asimilación de $\mathrm{CO}_{2}$ se reduce aún más debido a la disminución de la resistencia aerodinámica debido a una superficie más fría y, en consecuencia, menor velocidad convectiva en la superficie. En general la apertura estomatal es el proceso dominante y, por tanto, el efecto neto debido a la sombra de una nube delgada es un incremento en la asimilación de $\mathrm{CO}_{2}$ de la vegetación. 
Debido a que la división directa-difusa de la radiación modifica los flujos de superficie bajo las nubes, se estudia si estas modificaciones en la superficie impactan las propiedades de la capa límite y de los cúmulos someros. Los resultados indican que los cambios en los flujos de superficie no son suficientemente fuertes como para repercutir en la capa límite debido a dos razones. La primera se debe a que el aumento (disminución) del calor latente (sensible) es relativamente pequeño, de hasta el $10 \%$ para el calor latente. Además, el rango de grosor de nubes en el cual estos efectos aparecen es muy limitado, y las nubes de ese grosor no son suficientemente frecuentes a lo largo de los experimentos numéricos. De hecho, la cobertura de nubes nunca sobrepasa el $20 \%$ en este caso.

En el Capítulo 4 se investigan las interacciones nube-vegetación en un clima y capa límite diferentes a los anteriores. En este capítulo de diseña un nuevo caso LES a partir de la extensa base de datos reunida durante la campaña experimental del proyecto DynamicsAerosol-Chemistry-Cloud Interactions in West Africa (DACCIWA) con la contribución de quien escribe. Parte de la campaña consistió en observaciones intensivas del estado termodinámico de la capa límite, las nubes de capa límite y las condiciones de superficie en Benín, en la parte sur de África Occidental. El caso LES diseñado reproduce una sucesión de eventos frecuentemente observada durante la campaña de medidas: la existencia durante la noche y primeras horas del día de una cubierta de nubes estratocúmulos inicialmente baja y homogénea sobre tierra, alzándose la base de las nubes gradualmente tras la salida del sol. Después, la cubierta de nubes adelgaza y se rompe, creando heterogeneidades dinámicas en la superficie. Por la tarde las nubes evolucionan a cúmulos someros. Múltiples procesos actúan durante esta transición de unas pocas horas. Basado en el caso LES diseñado, se describe y cuantifica la transición de estratocúmulos nocturnos a cúmulos someros por la tarde. Se cuantifica la transición en términos de perfiles verticales termodinámicos, radiativos y turbulentos. Asimismo, se describe el desacoplamiento entre la capa de nubes y la superficie, la evolución y expansión de la capa de nubes y capa de inversión además de la evolución de niveles verticales relevantes dinámicamente como la altura de menor flujo de flotabilidad (buoyancy) y la cima de la capa sub-nube (subcloud layer top).

Para comprender mejor la transición se lleva a cabo un balance de columna integrada de agua líquida que revela los procesos físicos causantes del adelgazamiento y consecuente ruptura de la cubierta de estratocúmulos. El enfriamiento radiativo de onda larga en la cima de la nube domina el engrosamiento de la capa nubosa previo a la salida del sol con valores de $40 \mathrm{~g} \mathrm{~m}^{-2} \mathrm{~h}^{-1}$. La subsidencia muestra una contribución negativa y prácticamente constante de alrededor de $20 \mathrm{~g} \mathrm{~m}^{-2} \mathrm{~h}^{-1}$. Otros procesos tienen una contribución menor durante la noche. El resultado durante la noche es por tanto un engrosamiento de la capa nubosa de unos $20 \mathrm{~g} \mathrm{~m}^{-2} \mathrm{~h}^{-1}$, equivalente a un crecimiento de un $15 \%$ por hora. Tras la salida del sol la radiación de onda corta afecta el balance en dos maneras: debilita el enfriamiento radiativo de onda larga en la cima de la nube y aumenta los flujos de superficie, incrementando así el arrastre con incorporación de 
aire de la troposfera libre a la capa límite (entrainment). Tanto el debilitamiento del enfriamiento como el aumento de flujos de superficie contribuyen a un adelgazamiento de la capa de nubes. Este adelgazamiento comienza unos 75 minutos después de la salida del sol. Se realizan experimentos LES adicionales con perfiles de viento representativos de la corriente de chorro a un nivel cercano a la superficie (low level jet) observado. La cizalladura de viento asociada a la corriente de chorro dificulta el engrosamiento de la capa nubosa durante la noche y acelera el adelgazamiento y ruptura de la cubierta de estratocúmulos mediante dos procesos: el aumento del arrastre con incorporación de aire debido al efecto dinámico directo de la cizalladura de viento en la cima de la capa nubosa, y mayores flujos de superficie después del amanecer debido a una capa nubosa más delgada. Asimismo, la cizalladura de viento expande la capa nubosa y la capa de inversión y acelera el desacoplamiento entre la capa nubosa y la superficie. Además, describimos la evolución temporal del ratio de los flujos de flotabilidad y humedad entre la cima de la capa sub-nube y la superficie. Estos ratios se utilizan frecuentemente en parametrizaciones en modelos de escalas mayores para representar la dinámica de la capa límite. El ratio de flotabilidad muestra una reducción lineal después de la salida del sol estabilizándose en valores de -0.2 hacia as 12:30 HL. Este valor es típico de capas límite convectivas. El ratio de humedad decrece de 0.8 a 0.7 antes de la ruptura de la capa nubosa, indicativo de una humidificación neta de la capa límite. Después de la ruptura de la capa nubosa el ratio de humedad crece, indicando un secado neto en la capa sub-nube. La cizalladura de viento acelera las tendencias tanto del ratio de flotabilidad como del de humedad.

El objetivo del Capítulo 5 es estudiar el impacto de la transferencia radiativa condicionada por las nubes. Se analiza y cuantifican las consecuencias de utilizar un modelo radiativo unidimensional (1D) comparado con un innovador modelo tridimensional (3D) acoplados a un modelo de superficie terrestre. Éste es una de las primeras intentos de simular un caso de cúmulos someros con vegetación activa acoplada a un modelo radiativo 3D, ya que estos modelos radiativos implican a menudo un gran gasto computacional. El esquema 3D empleado ha sido desarrollado recientemente y resuelve de manera relativamente rápida la transferencia radiativa de radiación difusa en 10 direcciones y la de radiación directa en 3 , en lugar de las 2 en el eje vertical habituales tanto para radiación directa como difusa. Las simulaciones LES con esquemas radiativos 1D y 3D representan un caso de cúmulos someros sobre la selva Amazónica. Analizamos los efectos radiativos en la superficie y en la respuesta de la vegetación, así como las diferencias en la turbulencia en la capa límite y en la estructura y dinámica de las nubes. Los resultados muestran que la radiación 3D produce efectos observados experimentalmente como valores de radiación localmente mayores que en casos sin nubes debido a reflejos laterales de las nubes. La radiación adicional debido a estos efectos puede ser equivalente a hasta el $60 \%$ de la radiación recibida durante la hora de mayor irradiación diaria, esto es, entre las 11:30 y 12:30 HL. La asimilación de $\mathrm{CO}_{2}$ por la vegetación y el flujo de calor latente en la superficie 
muestran fenómenos similares. Además, 3D muestra mayor variedad y combinaciones de valores de radiación directa y difusa en la superficie y, por tanto, mayor variabilidad del flujo de calor latente. El experimento 3D produce mayor energía cinética de turbulencia (turbulence kinetic energy) y mayores corrientes de aire ascendente en la mitad superior de la capa límite a partir de las 13 HL. Estos resultados coinciden con flujos turbulentos de flotabilidad y humedad mayores dentro de la capa nubosa. Estos aumentos son debidos a la inclinación de la sombra de las nubes, que desacopla la zona sombreada del origen de la corriente de aire ascendente sobre la que la nube se forma. La mayor actividad nubosa y de flujos produce una capa nubosa más húmeda. Los efectos 3D de la radiación provocan nubes de mayor área después de las 13 HL. Mientras en 1D el tamaño transversal de la nube se estabiliza en unos $300 \mathrm{~m}$, en 3D el tamaño medio de las nubes continúa creciendo hasta los $500 \mathrm{~m}$ debido a la inclinación de la sombra de las nubes. Este aumento en tamaño concuerda con las mayores velocidades verticales y transporte turbulento de humedad presentes dentro de las nubes.

Esta tesis presenta investigaciones pioneras en las interacciones entre las nubes de capa límite y la vegetación activa. Integra conocimiento de diferentes disciplinas en un sistema y aborda cuestiones relacionadas con los efectos potenciales que nubes y plantas ejercen entre sí a través de fenómenos radiativos y turbulentos. Diseñamos experimentos LES para desligar las numerosas interacciones presentes y aportar los primeros hallazgos de las relaciones descubiertas. Los resultados muestran que la aparición, mantenimiento y disolución de las nubes en la capa límite están fuertemente ligadas a la actividad de la vegetación y la respuesta de ésta a la sombra de las nubes. A la vez, concluimos que los procesos relacionados con la fotosíntesis como la apertura estomatal están fuertemente condicionados por la dinámica de nubes. Además, proponemos direcciones para futuras investigaciones con la intención de progresar en la comprensión de las interacciones entre vegetación y nubes en la capa límite como un sistema acoplado. 


\section{Laburpena}

Tesi honen helburua muga-geruzako hodeien eta landaretzaren arteko interakzioak deskribatzea eta kuantifikatzea da. Muga-geruzako hodeiek garapen bertikala erakutsi dezakete, kumuluak direla kasu. Beste batzuk, estratokumulusak esate baterako, horizontalki homogeneoak dira. Bestalde, lurrazalaren eta atmosferaren arteko ur lurrin eta $\mathrm{CO}_{2}$ fluxuak landaretzaren fotosintesiaren bidez kontrolatuta daude. Ondorioz, landare aktibitateak lurrazaleko balantze energetikoa menperatzen du eta, beraz, energi erabilgarria azaleko bero sentikor eta bero sor fluxuetan banatzen du. Aipatutako bero sentikor eta bero sor fluxuek muga-geruzako turbulentzia kontrolatzen dute eta, ondorioz, honen bidez garraiatutako beroa eta hezetasuna. Gainera, tenperatura eta hezetasuna funtsezko aldagaiak dira muga-geruzako hodeien eraketa eta garapenerako. Aldi berean hodei hauek energia erabilgarria murrizten dute lurrazalean, fotosintesia eta azaleko fluxuak aldatuz. Zerua guztiz estali gabeko egoera hodeitsuetan, kumulu edo estratokumulu apurtuak esate baterako, hodeiek heterogeneotasun dinamikoak sortzen dituzte lurrazalean itzalen bidez. Tesi honen helburu nagusia landaretza eta hodeien arteko bi-noranzkoko efektuak argitzea eta prozesu nagusiak kuantifikatzea da hainbat ekosistema eta hodei egoeratan.

Gure metodoa simulazio numerikoak dira, non fluidoen lege fisikoak ebazten diren eta fotosintesia eta estoma irekitzea modelo mekanistiko baten bidez ordezkatzen diren. Simulazio idealizatu hauek Large Eddy Simulation (LES) edo zurrunbilo handien simulazio deitutako teknikarekin burutzen dira. LES-en airearen mugimendu turbulentoak era esplizituan simulatzen dira, hodeien dinamika barne. LES modeloari lurrazal modelo bat akoplatzen zaio hostoen estoma irekitzea eta fotosintesia simulatuz. Lurrazal modelo honek fotosintesia eta, beraz, bero sor fluxu balio desberdinak ematen ditu hainbat ingurune-aldagai kontuan hartuz, besteak beste: airearen hezetasuna, tenperatura eta $\mathrm{CO}_{2}$ kontzentrazioa, lurzoruaren hezetasuna, uhin motzeko erradiazioa eta haizea.

2. Kapituluan gaur egungo mesoeskalako modelo eta parametrizazioen gaitasuna kumuluen efektu dinamiko eta erradiatiboak simulatzeko aztertzen da, eta baita efektu hauen ondorioz landaretzaren erantzuna. Horretarako uda bukaerako ohiko egun bat simulatzen dugu mesoeskalako modelo bat lau konfigurazio desberdinekin eta bi modelo LES ezberdinekin alderatuz. Mesoeskalako simulazioak ez dira gai hodeien sorrera simulatzeko ez denborari, tokiari, altuerari ezta indarrari dagokionez. Gainera, hodei geruza beroegia (2 K-raino) eta lehorregia ( $2 \mathrm{~g} \mathrm{~kg}^{-1}$-raino) erakusten dute. Era berean, hodeiek erradiazioan eragindako aldaketak ez dira egokiak mesoeskalako simulazioetan: uhin motzeko erradiazioan aldakortasun gehiegi erakusten dute, hodeien ur-likido-zutabe (liquid water path) aldakortasun handiegia dela eta. Uhin motzeko erradiazioaren banaketa osagai zuzen eta difusoan aztertzen dugu, mesoeskalako simulazioek hodeiek sortutako erradiazio difusoaren \%50 gutxiesten dutela aurkituz. Emaitza hauek direla eta tesi honen gainerako ikerketan LES simulazioak soilik erabiltzen dira. 
Kumulu hodeien efektu erradiatiboek landaretzan duten efektua sakonago aztertzen da 3. Kapituluan. Hor, landaretzaren erantzunak aztertzen dira hodeiek uhin motzeko osagai zuzen eta difusoan daukaten eragina kontutan hartuz. Landaretzak era eraginkorrago batean erabiltzen du erradiazio difusoa, azken hau modu homogeneoago batean banatzen baita goiko hostoetatik behera landaretzan barne. Kapitulo honetako simulazioek zera erakusten dute: hodei finen azpian zeru garbiaren azpian baino fotosintesi handiagoa eta ondorioz, bero sor fluxu handiagoak agertzen dira. Honen azalpena hurrengoa da: nahiz eta hodeiek erradiazio totala murrizten duten, erradiazio difusoaren hazkundea garrantzitsuagoa da eta ondorioz, fotosintesia areagotzen da. Jarraian, aurkikuntza honek muga-geruzan eta hodeietan duen inpaktua ikertzen da. Emaitzek erakusten dute hodei finek eragindako aldaketak ez direla behar bezain indartsuak eta muga-geruza eta hodeien ezaugarriak ez direla era adierazgarri batean eraldatzen. Izan ere, alde batetik bero sor fluxuaren handitzea erradiazio difusoa dela eta \%10ekoa da gehienez. Bestetik, hodei finak ez dira maiz simulazioan agertzen, hodei estaldura gehienez $\% 20$ baita.

Tesi honen 4. Kapituluan LES kasu berri bat diseinatzen da Dynamics-AerosolChemistry-Cloud Interactions in West Africa (DACCIWA) proiektuko behaketetan oinarrituz. Kasuak DACCIWA behaketetan maiz gertatutako sekuentzia simulatzen du: estratokumulo baxuak gauean landarediaren gainean, hodei oinen igoera eguna argitu bezain laster, eta hodei geruzaren haustura eguerdi inguruan, arratsaldean kumulu hodeiak agertuz. LES simulazioan oinarrituta hodei trantsizioa deskribatu eta kuantifikatzen da erradiazio, termodinamika eta turbulentiza profil bertikalen bidez. Gainera, hodeien desakoplamendua lurrazaletik, hodei geruzaren, inbertsio geruza eta maila bertikal garrantzitsuen garapena deskribatzen dira. Trantsizioa hobeto ulertzeko ur-likido-zutabe balantzea burutzen dugu, prozesu fisiko garrantzitsuenak aurkitzeko. Gauez uhin luzearen bidez gertatutako hozte erradiatiboak hodei geruza loditzen du, \%15 orduko inguru. Eguzkia atera eta gero uhin motzeko erradiazioak hozte hori txikitzen du, arrastea (muga-geruza gaineko airea muga-geruzan sartzea) handitzen du, eta azal fluxuak handitzen ditu. Prozesu hauek hodei geruza mehetzen dute egunean zehar estratokumulus geruza apurtu arte. Bi esperimentu gehiago egiten dira haize zizailaduraren efektuak ikasteko: arrastea handitzen du, hodei geruza zabaldu eta honen eta lurrazalaren arteko desakoplamendua bizkortzen du.

5. Kapituluan dimentsio bakarreko edo hiru dimentsioko modelo erradiatiboak erabiltzearen ondorio fisikoak ikasten dira Amazonaseko kumulu kasu batean. Modelo tridimensionalek (3D) askoz baliabide informatiko gehiago behar dituzte. Berriki garatutako modelo batek, hemen erabilitakoa, erlatiboki azkar burutzen ditu kalkulo tridimentsionalak. Lurrazala modelora akoplatuz, modelo erradiatiboak lurrazalean, muga-geruzan eta hodei ezaugarrietan dauzkan efektuak aztertzen dira. 3D esperimentuak errealitatean behatutako efektuak erakusten ditu, hala nola, lurrazaleko erradiazio totalaren hazkunde lokala agertzen da hodei alboetako erreflexuak direla eta. 3D esperimentuak turbulentzia gehiago sortzen du 13 00tatik ordu lokala (OL) aurrera muga-geruzaren goikaldean, flotaga- 
rritasun eta hezetasun fluxu handiagoekin batera. Hazkunde hau, hodeiaren itzala hodei azpitik edo hodeia sortzen den fluxuetatik urrun egotearen ondorio da. Egoera honek hodei handiagoak sorrarazten ditu 1300 tatik OL aurrera: 3D esperimentuak horizontalean batezbesteko 500 m-tako hodeiak erakusten ditu arratsaldean, 1D-k 300 m-takoak erakusten duen bitartean. Aurrekoaz gain, 3D-ko hodeiek hezetasun eta flotagarritasun garraio handiagoa ere erakusten dute. 



\section{Supplementary material}

This material is published as supplementary material in:

Pedruzo-Bagazgoitia, X., Ouwersloot, H. G., Sikma, M., van Heerwaarden, C. C., Jacobs, C. M. J., and Vilà-Guerau de Arellano, J. (2017). Direct and diffuse radiation in the shallow cumulus-vegetation system: Enhanced and decreased evapotranspiration regimes. Journal of Hydrometeorology, 18(6):1731-1748

\section{S1 Supplementary material for Chapter 3: Canopy radiative transfer scheme: Two big leaf A-gs}

The main concepts of the canopy radiative transfer scheme are sketched in Figure S1.1. The model takes into account the following processes: transmission of radiation by leaves, scattering of direct radiation by leaves, absorption of radiation by leaves and upward reflection of direct and diffuse radiation by ground surface. The calculation of gross primary productivity and conductance for the whole canopy is carried out in three steps. Firstly, the potential leaf absorption of direct and diffuse light at leaf level is calculated at different LAI-dependent heights, taking into account secondary (direct light scattered by leaves) and tertiary (diffuse and direct light reflected by the ground as upward diffuse radiation) diffuse sources. All direct light not initially absorbed by leaves is assumed to be either lost or converted by ground reflection and leaf scattering processes into diffuse radiation. The diffuse and direct absorbed quantities are redistributed in radiation absorption values by sunlit and shaded leaves at each level, leading to a net photosynthesis rate and stomatal conductance value for each level. Finally, the scaling up from leaf to canopy level is carried out. There, the contributions of each level to total photosynthesis rate are added using the Gaussian integration method described in Goudriaan (1986). 


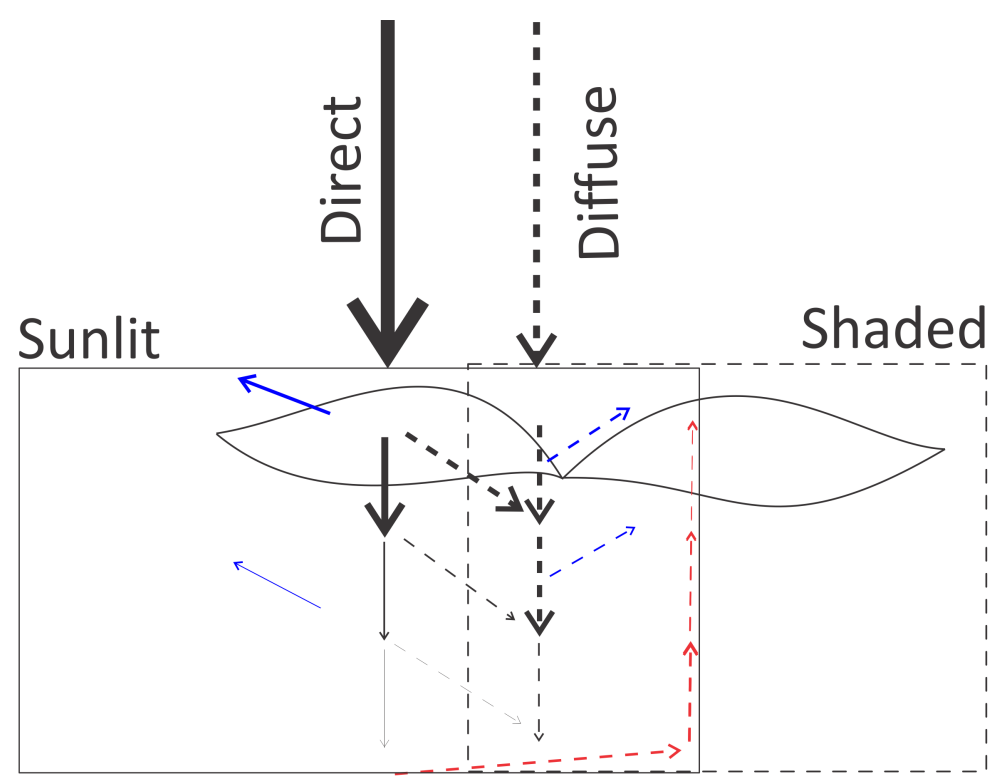

Figure S1.1: Representation of the transfer of direct and diffuse radiation inside the canopy. The three levels at which primary productivity and $\mathrm{CO}_{2}$ stomatal conductance are calculated are shown, as well as the sunlit (full line box) and shaded leave (dashed line box) distinction and the radiation lost by reflection and scattering effects (blue lines). Full and dashed arrows represent direct and diffuse light, respectively. Interestingly, a stronger attenuation in radiation is shown for the direct radiation compared to the diffuse one. The sketch shows the secondary diffuse component coming from scattered direct radiation at each level (dashed black arrows going from direct to diffuse light), and the tertiary diffuse component originated after the reflection of the direct beam in the ground surface (red dashed lines).

\section{Step 1: PAR profiles and absorption at leaf level at a given canopy level}

In agreement with literature (Alados and Alados-Arboledas, 1999; Spitters et al., 1986), we obtain approximated values for direct and diffuse PAR at top of the canopy by $P A R_{d i r}^{T o C}=$ $0.5 S W_{d i r}$ and $P A R_{d i f}^{T o C}=0.5 S W_{d i f}$. In general, radiation is reduced exponentially when it penetrates into the canopy according to the Beer-Lambert law as follows:

$$
S W(D)=(1-c) S W^{T o C} e^{-k i L A I(D)}
$$

where $S W^{T o C}$ stands for the radiation at the top of the canopy, $k$ is the extinction coefficient and $c$ is a factor accounting for losses of radiation due to scattering or reflection. $\operatorname{iLAI}(D)\left[\mathrm{m}_{\text {leaf }}^{2} \mathrm{~m}_{\text {ground }}^{-2}\right]$ is calculated by iLAI $=$ LAI $D$ and represents the accumulated LAI at depth $D$, where $D$ is a value between 0 (canopy top) and 1 (canopy bottom) giving the fraction of total LAI that is above that level. Note that $D$ will only be equal to the fraction of physical depth if the LAI is uniformly distributed over all the canopy layers. Here, $S W(D)$ as well as $S W^{T o C}$ are given in $\mathrm{W} \mathrm{m}$ ground $^{-2}$. 
The extinction coefficients for direct and diffuse light are obtained as follows:

$$
k_{d i f}=k_{d i f b l} \sqrt{1-\sigma}=0.8 \sqrt{1-\sigma}
$$

and

$$
k_{d i r}=k_{d r b l} \sqrt{1-\sigma}=\frac{0.5}{\sin \beta} \sqrt{1-\sigma}
$$

where $k_{d i f}$ and $k_{d i r}\left[\mathrm{~m}_{\text {ground }}^{2} \mathrm{~m}_{\text {leaf }}^{-2}\right]$ are the extinction coefficients for diffuse $($ dif $)$ and direct (dir) components of PAR respectively, and the additional $b l$ subscript stands for the extinction coefficient of "black leaves" not transmitting nor reflecting light, but only absorbing (Goudriaan, 1977). $\beta$ is the solar elevation angle above the horizon. Only scattering in the vertical direction is considered, represented by $\sigma$ and set to 0.2 (Goudriaan, 1977; Spitters, 1986). Note that the extinction coefficients are not dimensionless. It must be noted that $k_{d i r}$ does not give the extinction rate of direct light, but the extinction rate of radiation at a certain level due to the direct PAR reaching the top of the canopy. This amount of radiation present at that level may be pure direct or have a secondary or tertiary diffuse component if on the way it was scattered by leaves or the ground, respectively. $k_{d i r}$ stands for the extinction rate of the sum of primary direct radiation and the secondary diffuse radiation created when direct radiation is scattered, while $k_{d r b l}$ only takes into account the direct component of radiation.

The amount of PAR is reduced when it penetrates into the canopy, very similarly to how it was stated for radiation in Eq. (S1.1). The profile of available PAR [W m $\left.\mathrm{m}_{\text {ground }}^{-2}\right]$ inside the canopy is given, as stated by Spitters (1986) (Eqs. 3 and 4), by:

$$
\begin{gathered}
P A R_{d i f}^{T *}(D)=(1-\rho) P A R_{d i f}^{T o C} e^{-k_{d i f} i L A I(D)} \\
P A R_{d i r}^{T *}(D)=\left(1-\rho_{d i r}\right) P A R_{d i r}^{T o C} e^{-k_{d i r} i L A I(D)}
\end{gathered}
$$

$\rho$ stands for the reflection coefficient for horizontally distributed green leaves and for visible light (Goudriaan (1977), Eq. 2.21), and $\rho_{\text {dir }}$ for the reflection coefficient for spherically distributed green leaves under direct visible radiation. $P A R_{d i f}^{T o C}$ and $P A R_{d i r}^{T o C}$ give the amount of diffuse and direct radiation, respectively, at the top of the canopy. The reflection coefficients used above are defined as:

$$
\begin{gathered}
\rho=\frac{1-\sqrt{1-\sigma}}{1+\sqrt{1-\sigma}} \\
\rho_{\text {dir }}=\rho \frac{2}{1+1.6 \sin (\beta)}=\frac{1-\sqrt{1-\sigma}}{1+\sqrt{1-\sigma}} \frac{2}{1+1.6 \sin (\beta)}
\end{gathered}
$$

For diffuse radiation a horizontal leaf distribution is used due to the isotropy of diffuse light. In other words, there is no direction in which diffuse light would not arrive perpendicularly to the leaf, regardless of the distribution (Goudriaan, 1977; Spitters, 1986). For 
direct radiation, however, a spherical distribution is assumed and there is a certain angle depending on the leaf orientation with which radiation hits the leaves. The reflection dependency on the sun angle is given by the extra factor in $\rho_{d i r}$ that was not included in $\rho$.

Equations (S1.4) and (S1.5) can be extended by taking into account the light reflected by the ground, which is significant under low LAI. Actually, (Goudriaan, 1977) stated that above a LAI of 2 the influence of the soil surface can be practically neglected. In general, the profile of radiation reflected by the ground is given by:

$$
I_{\text {refl }}(D)=(1-r e f) I_{\text {ground }} e^{-k(L A I-i L A I(D))}
$$

where $I_{\text {ground }}$ is the radiation reflected by the ground and is calculated by: $I_{\text {ground }}=$ $a I^{T o C} e^{-k L A I}$, where $a$ is the albedo of the surface.

Using this approach for both direct and diffuse radiation we obtain the following profiles :

$$
\begin{aligned}
& P A R_{d i f}^{r e f l}(D)=(1-\rho) P A R_{d i f}^{\text {ground }} e^{-k_{d i f}(L A I-i L A I(D))} \\
& P A R_{d i r}^{r e f l}(D)=(1-\rho) P A R_{d i r}^{\text {ground }} e^{-k_{d i f}(L A I-i L A I(D))}
\end{aligned}
$$

where $P A R_{\text {dif }}^{\text {ground }}=a P A R_{\text {dif }}^{T o C} e^{-k_{d i f} L A I}$ and $P A R_{\text {dir }}^{\text {ground }}=a P A R_{d i r}^{T o C} e^{-k_{d i r} L A I}$ represent the radiation reflected by the ground due to the diffuse and direct radiation at the top of the canopy, respectively. $P A R_{d i f}^{r e f l}(D)$ and $P A R_{d i r}^{r e f l}(D)$ can be seen as sources of diffuse light going from the surface upwards with magnitude decreasing exponentially with height. The albedo of the ground for grass is estimated as $a=0.25$.

Taking this into account, the extended Equations (S1.4) and (S1.5) read:

$$
\begin{aligned}
& P A R_{d i f}^{T}(D)=P A R_{d i f}^{T *}(D)+P A R_{d i f}^{r e f l}(D) \\
& P A R_{d i r}^{T}(D)=P A R_{d i r}^{T *}(D)+P A R_{d i r}^{r e f l}(D)
\end{aligned}
$$

The second terms on the RHS in Eqs. (S1.11) and (S1.12) account for the radiation that is reflected by the ground surface.

It must be recalled that the PAR at the top of the canopy already has diffuse and direct components calculated by the delta-Eddington method. Thus, the outcome of Eqs. (S1.11) and (S1.12) is not necessarily the profile inside the canopy of diffuse and direct PAR respectively. Instead, $P A R_{d i f}^{T}(D)$ must be understood as the amount of (diffuse) PAR at normalized canopy depth $D$ due to the diffuse PAR present at the top of the canopy, and $P A R_{d i r}^{T}(D)$ as the amount of (diffuse and direct) PAR at normalized canopy depth $D$ due to the direct PAR present at the top of the canopy. In fact, Equation 
(S1.12) gives the sum of direct, secondary and tertiary diffuse PAR at every level due to the incoming direct PAR at the top of the canopy. Therefore, the diffuse PAR at a level $D$ will have contributions from both $P A R_{d i r}^{T}(D)$ and $P A R_{d i f}^{T}(D)$, while the direct PAR is a fraction of $P A R_{d i r}^{T}(D)$ at that level, since some of the PAR accounted for is secondary or tertiary diffuse.

The total profile for PAR direct radiation is given by Equation 5 in Spitters (1986):

$$
\begin{aligned}
P A R_{d i r}^{p r o f}(D) & =\left(1-\rho_{d i r}\right)(1-\sigma) P A R_{d i r}^{T o C} e^{-k_{d r b l} i L A I(D)} \\
& \approx(1-\sigma) P A R_{d i r}^{T o C} e^{-k_{d r b l} i L A I(D)}
\end{aligned}
$$

which only considers the direct PAR present at each level due to the direct PAR at the top of the canopy, without considering the secondary diffuse radiation nor any radiation reflected by the ground surface. The factor $(1-\sigma)$ accounts for the fact that some of the available direct radiation is scattered by the leaves. The approximation done in the second equality is due to the fact that $\rho_{\text {dir }}$ is of the order of 0.05 . Therefore, the factor giving the losses due to reflection and scattering can be approximated to $(1-\sigma)$. The exponential $e^{-k_{d r b l} i L A I(D)}$ can also be understood as the fraction of sunlit leaves at that level $D$.

Then, the diffuse PAR profile is given, as suggested by Spitters (1986) by:

$$
P A R_{\text {dif }}^{\text {prof }}(D)=\underbrace{P A R_{d i f}^{T}(D)}_{\begin{array}{c}
\text { primary diffuse and } \\
\text { diffuse ground-reflected }
\end{array}}+\underbrace{P A R_{\text {dir }}^{T}(D)-P A R_{\text {prof }}^{\text {prof }}(D)}_{\begin{array}{c}
\text { secondary diffuse and } \\
\text { direct ground-reflected }
\end{array}}
$$

A graphical example of Eqs. (S1.13) and (S1.14) is shown in Figure S1.2a.

In general, the absorption of irradiance per unit leaf area $H(D)\left[\mathrm{W} \mathrm{m}\right.$ leaf $\left.^{-2}\right]$ is a fraction of the irradiance arriving $I(D)$ given in Equation (S1.1), and it can be calculated as:

$$
\begin{aligned}
H(D) & =-\frac{d I(D)}{d i L A I}=(1-r e f) k I^{T o C} e^{-k i L A I(D)} \\
& =k I(D)
\end{aligned}
$$

The absorption at a level can be calculated as the difference between the radiation reaching that level and the radiation reaching the next (inferior) level. This definition, in fact, is nothing but the discrete definition of a derivative. Note that for this absorption and all the absorptions defined here the units are $\mathrm{W} \mathrm{m}_{\text {leaf }}^{-2}$, while the previously defined PAR's are calculated in $\mathrm{W} \mathrm{m}_{\text {ground }}^{-2}$. 

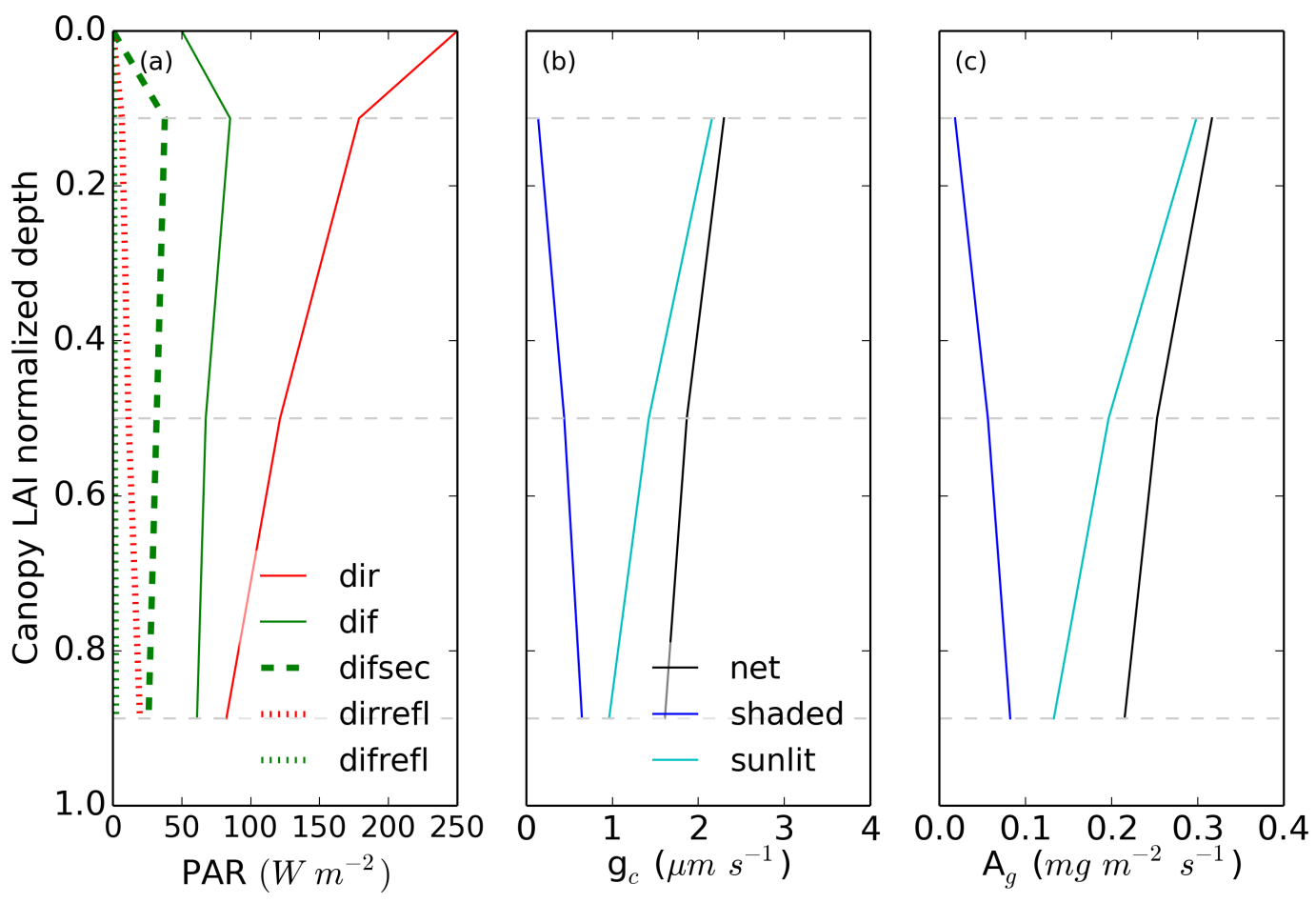

Figure S1.2: In-canopy profiles for Photosynthetic Active Radiation (left), stomatal conductance $g_{c}$ (center) and primary productivity $A_{g}$ (right) for a canopy with LAI $=2$ and prescribed top-of-canopy radiation consisting on $P A R_{d i r}^{T o C}=250 \mathrm{~W} \mathrm{~m}^{-2}$ and $P A R_{d i r}^{T o C}=50 \mathrm{~W} \mathrm{~m}^{-2}$ at the equator and solar elevation angle $\beta=\frac{\pi}{2}$. The plot on the left shows the total amount of direct (solid red) and diffuse (solid green) PAR at each height. Red dotted and green dotted lines show direct and diffuse radiation, respectively, reflected by the ground. Green dashed line gives the secondary diffuse radiation at each level. Light and dark blue lines give the contribution of sunlit and shaded leaves, respectively, to the net values $g_{c}^{\text {net }}$ and $A_{g}^{\text {net }}$ in black. The three levels that are actually calculated and used in the model are shown by gray horizontal dashed lines.

The total absorption due to diffuse PAR at the top of the canopy per unit leaf area at a certain LAI-normalized depth D follows from Eqs. (S1.11) and (S1.15), and is given by:

$$
\begin{aligned}
H_{d i f}^{T}(D) & =-\left(\frac{d P A R_{d i f}^{T *}(D)}{d i L A I}+\frac{d P A R_{d i f}^{r e f l}(D)}{d(-i L A I)}\right) \\
& =(1-\rho) k_{d i f} P A R_{d i f}^{T o C} e^{-k_{d i f} i L A I(D)} \\
& +(1-\rho) k_{d i f} P A R_{d i f}^{\text {ground }} e^{-k_{d i f}(L A I-i L A I(D))} \\
& =k_{d i f} P A R_{d i f}^{T *}(D)+k_{d i f} P A R_{d i f}^{r e f l}(D)
\end{aligned}
$$

Note that in this case the second term has been differentiated over -iLAI. This is because the radiation reflected by ground travels upward, from the ground to the top of 
canopy.

Similarly, for the total absorption per unit leaf area due to direct PAR at the top of the canopy, combining Eqs. (S1.12) and (S1.15):

$$
\begin{aligned}
H_{d i r}^{T}(D) & =-\left(\frac{d P A R_{d i r}^{T *}(D)}{d i L A I}+\frac{d P A R_{d i r}^{r e f l}(D)}{d(-i L A I)}\right) \\
& =\left(1-\rho_{d i r}\right) k_{d i r} P A R_{d i r}^{T o C} e^{-k_{d i r} i L A I(D)} \\
& +(1-\rho) k_{d i f} P A R_{d i r}^{g r o u n d} e^{-k_{d i f}(L A I-i L A I(D))} \\
& =k_{d i r} P A R_{d i r}^{T *}(D)+k_{d i f} P A R_{d i r}^{r e f l}(D)
\end{aligned}
$$

The primary absorption per unit leaf area of the direct component of the direct PAR at the top of the canopy is given by:

$$
\begin{aligned}
H_{d i r}^{p r o f}(D) & =-\frac{d P A R_{d i r}^{p r o f}(D)}{d i L A I}=(1-\sigma) k_{d r b l} P A R_{d i r}^{T o C} e^{-k_{d r b l} i L A I(D)} \\
& =k_{d r b l} P A R_{d i r}^{\text {prof }}(D)
\end{aligned}
$$

Later we will be interested in knowing the amount of absorbed radiation by shaded and sunlit leaves separately, so we can finally add their contributions depending on the amount of sunlit and shaded leaves per layer. Therefore, it is interesting to calculate the amount of radiation absorbed at horizontal leaf level if we only take into account the direct light coming from the sun. We assume that the amount of radiation will not depend on LAI, since the beam would hit the leaf with equal intensity regardless of the number of leaves lying above. If all the direct radiation hitting a squared meter of ground was absorbed, this amount would be given by $(1-\sigma) P A R_{d i r}^{T o C}$. However, a fraction $e^{-k d r b l ~ L A I}$ reaches the ground and, therefore, it is not absorbed as direct radiation. Thus, the total direct radiation absorbed per meter square of ground is $(1-\sigma) P A R_{d i r}^{T o C}\left(1-e^{-k d r b l L A I}\right)$. The total area of sunlit leaves per meter square of ground $A_{\text {sun }}\left[\mathrm{m}_{\text {leaf }}^{2} \mathrm{~m}_{\text {ground }}^{-2}\right]$ can be calculated by integrating the already mentioned fraction of sunlit leaves at a level $D, e^{-k_{d r b l} i L A I(D)}$, over all canopy depth or, what is the same, over LAI. By doing so:

$$
A_{\text {sun }}=\int_{0}^{L A I} e^{-k_{d r b l} i L A I(D)} \mathrm{d} i L A I=\frac{1}{k_{d r b l}}\left(1-e^{-k_{d r b l} L A I}\right)
$$

Now, we can easily calculate the amount of radiation absorbed per sunlit leaf area by dividing the absorbed radiation per meter square ground by the area of sunlit leaves in a meter square ground: 


$$
H_{d i r}^{P P}=\frac{(1-\sigma) P A R_{d i r}^{T o C}\left(1-e^{-k d r b l L A I}\right)}{\frac{1}{k_{d r b l}}\left(1-e^{-k_{d r b l} L A I}\right)}=(1-\sigma) k_{d r b l} P A R_{d i r}^{T o C}
$$

The units of $H_{d i r}^{P P}$ are $\mathrm{W} \mathrm{m}_{\text {leaf }_{\mathrm{p}}}^{-2}$ where lea $f_{p}$ stands for leaves perpendicular to the beam, and therefore consistent with the absorbed quantities defined before. Note, as expected and stated by Spitters (1986), that "the intensity of the direct beam per unit leaf area does not change with canopy depth".

Now that we have expressions for direct and diffuse PAR absorptions at each level, we will calculate the absorption by sunlit and shaded leaves at each level. Shaded leaves will absorb primary, secondary and tertiary diffuse radiation, while sunlit leaves will additionally absorb the no scattered primary direct light.

To account for the radiation absorbed by shaded leaves per leaf area at any level we take difference between the total absorption at that level (diffuse light absorption accounting for ground reflection of diffuse light + direct light absorption accounting for secondary and tertiary diffuse absorption) minus the primary direct absorption at that level (without secondary or tertiary diffuse radiation source):

$$
H^{\text {shad }}(D)=\underbrace{H_{d i f}^{T}(D)}_{\begin{array}{c}
\text { primary diffuse and } \\
\text { diffuse ground-reflected }
\end{array}}+\underbrace{H_{d i r}^{T}(D)-H_{\text {dir }}^{\text {prof }}(D)}_{\begin{array}{c}
\text { secondary diffuse and } \\
\text { direct ground-reflected }
\end{array}}
$$

To obtain the total absorption of sun leaves $H^{\text {sun }}(D)$, we need to add the primary absorption per unit leaf area coming from the sun to the term obtained in Eq. (S1.21). This primary absorption term will depend on the angle between the incident beam and the leaf position and, therefore, on the leaf angle distribution of the canopy. Jacobs and de Bruin (1997) proposed a spherical leaf distribution (not preferred leaf orientation). Strictly speaking, an explicit calculation requires the integration over all the angles in order to account for the direct beam incident on every leaf. However, as shown in Goudriaan (1988), taking 3 angles is enough for an accurate estimation of assimilation by direct beam irradiation by means of a Gaussian integration. In this method, few points (three angles, in this case) are taken representing the whole canopy and they are added taking into account some predefined weighting factors (Goudriaan, 1986). The 3 angles are chosen such that the sinus of each of them are $\sin \left(\gamma_{1}\right)=0.1127, \sin \left(\gamma_{2}\right)=0.5000$, and $\sin \left(\gamma_{3}\right)=0.8873$ respectively. The weights are given as follows: $w_{1}=w_{3}=0.2778$, and $w_{2}=0.4444$. These same values will be used in the second Gaussian integration carried out in the upscaling in the Step 3 . Therefore, to obtain the absorbed radiation per leaf area by sunlit leaves with a certain incidence angle $\gamma_{i}$ :

$$
H^{\text {sun }}\left(D, \gamma_{i}\right)=H^{\text {shad }}(D)+H_{d i r}^{P P} \frac{\sin \left(\gamma_{i}\right)}{\sum_{i=1}^{3} w_{i} \sin \left(\gamma_{i}\right)}
$$


The last term in Eq. (S1.22) is divided over the product of all the weights with their respective sinus in order to fulfill the conservation of energy per square meter ground. Note that the units of $H^{\text {sun }}\left(D, \gamma_{i}\right)$ are $\mathrm{W} \mathrm{m}_{\text {leaf }_{\mathrm{i}}}^{-2}$, where leaf area is not perpendicular to the light beam but tilted with an inclination given by the angle $\gamma_{i}$.

\section{Step 2: Gross primary productivity and conductance by sunlit and shaded leaves at a given canopy height}

Once we have the radiation absorbed per unit leaf area by shaded leaves we can obtain the $\mathrm{CO}_{2}$ gross primary productivity $A_{g, l}^{\text {shad }}(D)\left[\mathrm{mg} \mathrm{s}^{-1} \mathrm{~m}_{\text {shaded leaf }}^{-2}\right]$ and $\mathrm{CO}_{2}$ stomatal conductance $g_{c, l}^{\text {shad }}(D)\left[\mathrm{mm} \mathrm{s}^{-1}\right]$ of shaded leaves per unit leaf area at leaf level using the the A-gs method (Jacobs and de Bruin, 1997; Ronda et al., 2001). In general the gross primary productivity at leaf level $A_{g, l}^{*}\left[\mathrm{mg} \mathrm{s}^{-1} \mathrm{~m}_{\text {shaded leaf }}^{-2}\right]$ under unstressed water situations is calculated as:

$$
A_{g, l}^{*}(D)=\left(A_{m}+R_{d}\right)\left[1-e^{-\frac{\alpha H(D)}{A_{m}+R_{d}}}\right]
$$

Here, $A_{m}$ stands for the primary productivity or photosynthetic rate at infinite light, $R_{d}$ $\left[\mathrm{mg} \mathrm{s}^{-1} \mathrm{~m}_{\text {shaded leaf }}^{-2}\right]$ represents the dark autrophic respiration and is calculated by $R_{d}=$ $0.11 A_{m}$. The light use efficiency is given by $\alpha$. Additionally, this value is corrected by a stress function $f(w)$, which accounts for the moisture content in the soil. This function is defined as:

$$
f(w)=\max \left[0, \min \left(1, \frac{\bar{w}-w_{\text {wilt }}}{w_{f c}-w_{w_{\text {wilt }}}}\right)\right]
$$

where $w_{f c}$ and $w_{w i l t}$ stand for the soil moisture content at field capacity and wilting point, respectively. The moisture-corrected gross primary productivity at leaf level is:

$$
\begin{aligned}
A_{g, l}(D) & =f(w) A_{g, l}^{*}(D) \\
& =f(w)\left(A_{m}+R_{d}\right)\left[1-e^{-\frac{\alpha H(D)}{A_{m}+R_{d}}}\right]
\end{aligned}
$$

Now, we can calculate the CO2 conductance at leaf level $g_{c, l}$ using the following expression:

$$
g_{c, l}(D)=g_{m i n, c}+\frac{A_{g, l}(D)}{\left(C_{s}-C_{i}\right)}
$$

where $C_{s}$ and $C_{i}$ are the external or atmospheric and internal or intercellular $\mathrm{CO}_{2}$ concentrations respectively. $g_{\text {min,c }}$ is the minimal cuticular conductance for carbon given by 
$g_{\text {min,c }}=\frac{g_{\min , w}}{1.6}$, where $g_{\text {min }, w}$ stands for the minimal cuticular conductance for water and assumed to be $g_{m i n, w}=2.5 \mathrm{~mm} \mathrm{~s}^{-1}$ (Ronda et al., 2001).

For a more detailed explanation on Eqs. (S1.23)-(S1.26) and definition of variables the reader is referred to Vilà-Guerau de Arellano et al. (2015).

To calculate the primary productivity at leaf level by shaded leaves $A_{g, l}^{\text {shad }}(D)$, Eq. (S1.25) must be used, but using $H^{\text {shad }}(D)$ instead of $H(D)$. After doing this, the expression for $A_{g, l}^{\text {shad }}(D)$ is given by:

$$
A_{g, l}^{\text {shad }}(D)=f(w)\left(A_{m}+R_{d}\right)\left[1-e^{-\frac{\alpha H^{\text {shad }}(D)}{A_{m}+R_{d}}}\right]
$$

For $\mathrm{CO}_{2}$ conductance of shaded leaves at leaf level $g_{c, l}^{\text {shad }}\left[\mathrm{mm} \mathrm{s}^{-1}\right]$, Eq. (S1.26) changes to:

$$
g_{c, l}^{\text {shad }}(D)=g_{\text {min }, c}+\frac{A_{g, l}^{\text {shad }}(D)}{\left(C_{s}-C_{i}\right)}
$$

The leaf-angle dependent $\mathrm{CO}_{2}$ gross primary productivities for sunlit leaves at leaf level $A_{g, l}^{\text {sun }}\left(D, \gamma_{i}\right)\left[\mathrm{mg} \mathrm{s}^{-1} \mathrm{~m}_{\text {leaf }}^{-2}\right]$, where leaf $\mathrm{i}_{\mathrm{i}}$ means a leaf with inclination given by $\gamma_{i}$, and $\mathrm{CO}_{2}$ conductance $g_{c, l}^{\text {sun }}\left(D, \gamma_{i}\right)\left[\mathrm{mm} \mathrm{s}^{-1}\right]$ are first calculated for each angle using analogous expressions to those in Eqs. (S1.27) and (S1.28) respectively. In these cases, the expressions read:

$$
\begin{gathered}
A_{g, l}^{\text {sun }}\left(D, \gamma_{i}\right)=f(w)\left(A_{m}+R_{d}\right)\left[1-e^{-\frac{\alpha H^{\operatorname{sun}}\left(D, \gamma_{i}\right)}{A_{m}+R_{d}}}\right] \\
g_{c, l}^{\text {sun }}\left(D, \gamma_{i}\right)=g_{m i n, c}+\frac{A_{g, l}^{\text {sun }}\left(D, \gamma_{i}\right)}{\left(C_{s}-C_{i}\right)}
\end{gathered}
$$

Afterwards, the gross primary productivitiy $A_{g, d}^{\text {sun }}(D)\left[\mathrm{mg} \mathrm{s}^{-1} \mathrm{~m}_{\text {leaf }}^{-2}\right]$ and conductance $g_{c, d}^{\text {sun }}(D)\left[\mathrm{mm} \mathrm{s}^{-1}\right]$ for sunlit leaves accounting for the spherical leaf distribution are obtained, adding each term according to the Gaussian weights $\left(w_{1}=w_{3}=0.2778, w_{2}=\right.$ 0.4444) and as explained in Goudriaan (1986):

$$
\begin{gathered}
A_{g, l}^{\text {sun }}(D)=w_{1} A_{g, l}^{\text {sun }}\left(D, \gamma_{1}\right)+w_{2} A_{g, l}^{\text {sun }}\left(D, \gamma_{2}\right)+w_{3} A_{g, l}^{\text {sun }}\left(D, \gamma_{3}\right) \\
g_{c, l}^{\text {sun }}(D)=w_{1} g_{c, l}^{\text {sun }}\left(D, \gamma_{1}\right)+w_{2} g_{c, l}^{\text {sun }}\left(D, \gamma_{2}\right)+w_{3} g_{c, l}^{\text {sun }}\left(D, \gamma_{3}\right)
\end{gathered}
$$

The weights are projecting each term from their different leaf orientations $\gamma_{i}$ on the perpendicular-to-beam leaf orientation. 
Once we have the gross primary productivity and conductance per leaf area of shaded and sunlit leaves separately, they are added taking into account the fraction of sunlit leaf area at that level $e^{-k_{d r b i} i L A I(D)}$. The gross primary productivity $A_{g}^{\text {net }}(D)\left[\mathrm{mg} \mathrm{s}^{-1} \mathrm{~m}_{\text {leaf }}^{-2}\right]$ and conductance $g^{\text {net }}(D)\left[\mathrm{mm} \mathrm{s}^{-1}\right]$, still per unit leaf area at one level are given by:

$$
\begin{gathered}
A_{g}^{\text {net }}(D)=A_{g, l}^{\text {sun }}(D) e^{-k_{d r b l} i L A I(D)}+A_{g, l}^{\text {shad }}(D)\left(1-e^{-k_{d r b l} i L A I(D)}\right) \\
g_{c}^{\text {net }}(D)=g_{c, l}^{\text {sun }}(D) e^{-k_{d r b l} i L A I(D)}+g_{c, l}^{\text {shad }}(D)\left(1-e^{-k_{d r b l} i L A I(D)}\right)
\end{gathered}
$$

A graphical example of Eqs. (S1.33) and (S1.34) is shown in Figures S1.2c and S1.2b, respectively. This Figure shows the increasing relevance of secondary and tertiary radiation with canopy depth.

\section{Step 3: Upscaling of gross primary productivity and conductance for canopy}

In order to obtain the values of $A_{g}$ and $g_{c}$ for the whole canopy per ground unit we use a 3 point Gaussian integration (Goudriaan, 1986). Three levels $D_{i}$ are taken such that $\operatorname{iLAI}\left(D_{1}\right)=0.1127 \mathrm{LAI}, i L A I\left(D_{2}\right)=0.5000 \mathrm{LAI}$, and $\operatorname{iLAI}\left(D_{3}\right)=0.8873 \mathrm{LAI}$ with three weights $v_{i}$ respectively (0.2778 LAI, $\left.0.4444 \mathrm{LAI}, 0.2778 \mathrm{LAI}\right)$ as used for the angle integration in Eqs S1.31 and S1.32 and explained in Eqs. 20 and 21 in Spitters (1986). This upscaling method using gaussian weights has also been adopted in the Integrated Forecasting System (IFS) by the European Centre for Medium-Range Weather Forecasts (ECMWF) (Boussetta et al., 2013). The final expression for gross primary productivity and carbon conductance for the whole canopy reads:

$$
\begin{aligned}
& A_{g}^{c a n}=\sum_{i=1}^{3} v_{i} A_{g}^{\text {net }}\left(D_{i}\right) \\
& g_{c}^{\text {can }}=\sum_{i=1}^{3} v_{i} g_{c}^{\text {net }}\left(D_{i}\right)
\end{aligned}
$$

Once $g_{c}^{c a n}$ is obtained, a more realistic value for the net $\mathrm{CO}_{2}$ flow into the plant at canopy level can be calculated by adding the influence of atmospheric processes, as usually done for evaporation processes at the surface. Here, $r_{a}$ represents the aerodynamic resistance to the plant uptake. Thus, the net $\mathrm{CO}_{2}$ assimilation at canopy level $A_{n}^{\text {can }}$ reads:

$$
A_{n}^{c a n}=\frac{C_{s}-C_{i}}{r_{a}+r_{\operatorname{veg}_{\mathrm{CO}_{2}}}}
$$

where $r_{v e g_{\mathrm{CO}_{2}}}=\frac{1}{g_{c}^{\text {can }}} \cdot r_{a}$ acts as a limiting factor for net $\mathrm{CO}_{2}$ into the plant, especially for low $r_{v e g_{\mathrm{CO}_{2}}}$ values. The reader may wonder why the values for primary productivity 
$A_{g}$ have been calculated if, eventually, a corrected value is obtained which only needs $g_{c}^{c a n}$. The reason is that by doing so, we are able to draw approximate in-canopy profiles of the primary productivity (and net $\mathrm{CO}_{2}$ assimilation rate, if needed, using $A_{n, l}(D)=$ $A_{g, l}(D)-R_{d}$ (Ronda et al., 2001). 


\section{References}

Ackerman, A. S., Kirkpatrick, M. P., Stevens, D. E., and Toon, O. B. (2004). The impact of humidity above stratiform clouds on indirect aerosol climate forcing. Nature, 432(7020):1014-1017.

Adler, B., Babić, K., Kalthoff, N., Lohou, F., Lothon, M., Dione, C., Pedruzo-Bagazgoitia, X., and Andersen, H. (2019). Nocturnal low-level clouds in the atmospheric boundary layer over southern west africa: an observation-based analysis of conditions and processes. Atmospheric Chemistry and Physics, 19(1):663-681.

Alados, I. and Alados-Arboledas, L. (1999). Direct and diffuse photosynthetically active radiation: measurements and modelling. Agricultural and Forest Meteorology, 93(1):27 - 38 .

Albertson, J. D., Katul, G. G., and Wiberg, P. (2001). Relative importance of local and regional controls on coupled water, carbon, and energy fluxes. Advances in Water Resources, 24(9):1103 - 1118. Nonlinear Propagation of Multi-scale Dynamics Through Hydrologic Subsystems.

Alton, P., North, P., and Los, S. (2007). The impact of diffuse sunlight on canopy light-use efficiency, gross photosynthetic product and net ecosystem exchange in three forest biomes. Global Change Biology, 13(4):776-787.

Arakawa, A. (2004). The cumulus parameterization problem: Past, present, and future. Journal of Climate, 17(13):2493-2525.

Arakawa, A., Jung, J.-H., and Wu, C.-M. (2011). Toward unification of the multiscale modeling of the atmosphere. Atmospheric Chemistry and Physics, 11(8):3731-3742.

Avissar, R. and Schmidt, T. (1998). An evaluation of the scale at which ground-surface heat flux patchiness affects the convective boundary layer using large-eddy simulations. Journal of the Atmospheric Sciences, 55(16):2666-2689.

Babić, K., Adler, B., Kalthoff, N., Andersen, H., Dione, C., Lohou, F., Lothon, M., and Pedruzo-Bagazgoitia, X. (2019). The observed diurnal cycle of low-level stratus clouds over southern west africa: a case study. Atmospheric Chemistry and Physics, 19(2):1281-1299.

Baker, M. B. and Latham, J. (1979). The evolution of droplet spectra and the rate of pro- 
duction of embryonic raindrops in small cumulus clouds. Journal of the Atmospheric Sciences, 36(8):1612-1615.

Baldocchi, D. and Ma, S. (2013). How will land use affect air temperature in the surface boundary layer? lessons learned from a comparative study on the energy balance of an oak savanna and annual grassland in california, usa. Tellus B, 65(0).

Baldocchi, D. D., Hutchison, B. A., and Matt, D. R.and McMillen, R. T. (1985). Canopy radiative transfer models for spherical and known leaf inclination angle distributions: A test in an oak-hickory forest. Journal of Applied Ecology, 22(2):539-555.

Barbaro, E., de Arellano, J. V.-G., Ouwersloot, H. G., Schröter, J. S., Donovan, D. P., and Krol, M. C. (2014). Aerosols in the convective boundary layer: Shortwave radiation effects on the coupled land-atmosphere system. Journal of Geophysical Research: Atmospheres, 119(10):5845-5863.

Barbaro, J. (2015). Interaction between aerosols and convective boundary-layer dynamics over land. PhD thesis, Wageningen University.

Bauer-Pfundstein, M. R. and Goersdorf, U. (2007). Target separation and classification using cloud radar doppler-spectra. In Proceedings of the 33rd Intern. Conf. on Radar Meteorology, volume 11.B2, pages 1-8, Cairns, Australia.

Bellon, G. and Stevens, B. (2012). Using the sensitivity of large-eddy simulations to evaluate atmospheric boundary layer models. Journal of the Atmospheric Sciences, 69(5):1582-1601.

Bessardon, G., Brooks, B., Abiye, O., Adler, B., Ajao, A., Ajileye, O., Altstätter, B., Amekudzi, L. K., Aryee, J. N. A., Atiah, W., Ayoola, M., Babic, K., Bärfuss, K., Bezombes, Y., Bret, G., Brilouet, P.-E., Cayle-Aethelhard, F., Danuor, S., Delon, C., Derrien, S., Dione, C., Durand, P., Fosu-Amankwah, K., Gabella, O., Groves, J., Handwerker, J., Kalthoff, N., Kohler, M., Kunka, N., Jambert, C., Jegede, G., Lampert, A., Leclercq, J., Lohou, F., Lothon, M., Medina, P., Pätzold, F., PedruzoBagazgoitia, X., Reinares, I., Sharpe, S., Smith, V., Sunmonu, L. A., Tan, N., and Wieser, A. (2019). A new high-quality dataset of the diurnal cycle of the southern west african atmospheric boundary layer during the monsoon season - an overview from the dacciwa campaign. In preparation.

Betts, A. K. (1973). Non-precipitating cumulus convection and its parameterization. Quarterly Journal of the Royal Meteorological Society, 99(419):178-196.

Betts, A. K., Goulden, M., and Wofsy, S. (1999). Controls on evaporation in a boreal spruce forest. Journal of Climate, 12(6):1601-1618.

Blossey, P. N., Bretherton, C. S., Zhang, M., Cheng, A., Endo, S., Heus, T., Liu, Y., Lock, A. P., de Roode, S. R., and Xu, K.-M. (2013). Marine low cloud sensitivity to an idealized climate change: The cgils les intercomparison. Journal of Advances in Modeling Earth Systems, 5(2):234-258. 
Böing, S. J., Jonker, H. J. J., Siebesma, A. P., and Grabowski, W. W. (2012). Influence of the subcloud layer on the development of a deep convective ensemble. Journal of the Atmospheric Sciences, 69(9):2682-2698.

Boucher, O., Randall, D., Artaxo, P., Bretherton, C., Feingold, C., Forster, P., Kerminen, V.-M., Kondo, Y., Liao, H., Lohmann, U., Rasch, P., Satheesh, S., Sherwood, S., Stevens, B., and Zhang, X.-Y. (2013). Clouds and aerosols. Technical report. .

Boussetta, S., Balsamo, G., Beljaars, A., Panareda, A.-A., Calvet, J.-C., Jacobs, C., Hurk, B., Viterbo, P., Lafont, S., Dutra, E., et al. (2013). Natural land carbon dioxide exchanges in the ECMWF Integrated Forecasting System: Implementation and offline validation. Journal of Geophysical Research: Atmospheres, 118(12):59235946 .

Bretherton, C. S., Blossey, P. N., and Uchida, J. (2007). Cloud droplet sedimentation, entrainment efficiency, and subtropical stratocumulus albedo. Geophysical Research Letters, 34(3).

Bretherton, C. S., Krueger, S. K., Wyant, M. C., Bechtold, P., Van Meijgaard, E., Stevens, B., and Teixeira, J. (1999a). A gcss boundary-layer cloud model intercomparison study of the first astex lagrangian experiment. Boundary-Layer Meteorology, 93(3):341-380.

Bretherton, C. S., Macvean, M. K., Bechtold, P., Chlond, A., Cotton, W. R., Cuxart, J., Cuijpers, H., Mhairoutdinov, M., Kosovic, B., Lewellen, D., Moeng, C.-H., Siebesma, P., Stevens, B., Stevens, D. E., Sykes, I., and Wyant, M. C. (1999b). An intercomparison of radiatively driven entrainment and turbulence in a smoke cloud, as simulated by different numerical models. Quarterly Journal of the Royal Meteorological Society, 125(554):391-423.

Bretherton, C. S. and Wyant, M. C. (1997). Moisture transport, lower-tropospheric stability, and decoupling of cloud-topped boundary layers. Journal of the Atmospheric Sciences, 54(1):148-167.

Brodersen, C. R., Vogelmann, T. C., Williams, W. E., and Gorton, H. L. (2008). A new paradigm in leaf-level photosynthesis: direct and diffuse lights are not equal. Plant, Cell ES Environment, 31(1):159-164.

Brown, A. R., Cederwall, R. T., Chlond, A., Duynkerke, P. G., Golaz, J.-C., Khairoutdinov, M., Lewellen, D. C., Lock, A. P., MacVean, M. K., Moeng, C.-H., Neggers, R. A. J., Siebesma, A. P., and Stevens, B. (2002). Large-eddy simulation of the diurnal cycle of shallow cumulus convection over land. Quarterly Journal of the Royal Meteorological Society, 128(582):1075-1093.

Cahalan, R. F., Oreopoulos, L., Marshak, A., Evans, K. F., Davis, A. B., Pincus, R., Yetzer, K. H., Mayer, B., Davies, R., Ackerman, T. P., Barker, H. W., Clothiaux, E. E., Ellingson, R. G., Garay, M. J., Kassianov, E., Kinne, S., Macke, A., O’hirok, 
W., Partain, P. T., Prigarin, S. M., Rublev, A. N., Stephens, G. L., Szczap, F., Takara, E. E., Várnai, T., Wen, G., and Zhuravleva, T. B. (2005). The i3rc: Bringing together the most advanced radiative transfer tools for cloudy atmospheres. Bulletin of the American Meteorological Society, 86(9):1275-1294.

Casso-Torralba, P., Vilà-Guerau de Arellano, J., Bosveld, F., Soler, M. R., Vermeulen, A., Werner, C., and Moors, E. (2008). Diurnal and vertical variability of the sensible heat and carbon dioxide budgets in the atmospheric surface layer. Journal of Geophysical Research: Atmospheres, 113(D12):n/a-n/a. D12119.

Chen, T., Rossow, W. B., and Zhang, Y. (2000). Radiative effects of cloud-type variations. Journal of Climate, 13(1):264-286.

Cheng, S. J., Bohrer, G., Steiner, A. L., Hollinger, D. Y., Suyker, A., Phillips, R. P., and Nadelhoffer, K. J. (2015). Variations in the influence of diffuse light on gross primary productivity in temperate ecosystems. Agricultural and Forest Meteorology, 201:98 110.

Cheng, S. J., Steiner, A. L., Hollinger, D. Y., Bohrer, G., and Nadelhoffer, K. J. (2016). Using satellite-derived optical thickness to assess the influence of clouds on terrestrial carbon uptake. Journal of Geophysical Research: Biogeosciences, 121(7):1747-1761. 2016JG003365.

Ching, J., Rotunno, R., LeMone, M., Martilli, A., Kosovic, B., Jimenez, P. A., and Dudhia, J. (2014). Convectively induced secondary circulations in fine-grid mesoscale Numerical Weather Prediction models. Monthly Weather Review, 142(9):3284-3302.

Chlond, A., Boehringer, O., Auerswald, T., and Mueller, F. (2014). The effect of soil moisture and atmospheric conditions on the development of shallow cumulus convection: A coupled large-eddy simulation-land surface model study. Meteorologische Zeitschrift, 23:491-510.

Conzemius, R. J. and Fedorovich, E. (2006). Dynamics of sheared convective boundary layer entrainment. part i: Methodological background and large-eddy simulations. Journal of the Atmospheric Sciences, 63(4):1151-1178.

Couvreux, F., Roehrig, R., Rio, C., Lefebvre, M.-P., Caian, M., Komori, T., Derbyshire, S., Guichard, F., Favot, F., D'Andrea, F., Bechtold, P., and Gentine, P. (2105). Representation of daytime moist convection over the semi-arid tropics by parametrizations used in climate and meteorological models. Quarterly Journal of the Royal Meteorological Society, 141(691):2220-2236.

Cox, P. M., Betts, R. A., Jones, C. D., Spall, S. A., and Totterdell, I. J. (2000). Acceleration of global warming due to carbon-cycle feedbacks in a coupled climate model. Nature, 408(6809):184-187.

Cuijpers, J. W. M. and Duynkerke, P. G. (1993). Large eddy simulation of trade wind cumulus clouds. Journal of the Atmospheric Sciences, 50(23):3894-3908. 
de Bruin, H. A. R. (1983). A model for the priestley-taylor parameter $\alpha$. Journal of Climate and Applied Meteorology, 22(4):572-578.

de Bruin, H. A. R., Jacobs, C. M. J., Jarvis, P. G., McNaughton, K. G., Milford, J., Kohsiek, W., Rowntree, P., Monteith, J. L., Gardiner, B., and Shuttleworth, W. J. (1989). Forests and regional-scale processes [and discussion]. Philosophical Transactions of the Royal Society of London. Series B, Biological Sciences, 324(1223):393406.

de Roode, S. R. and Duynkerke, P. G. (1996). Dynamics of cumulus rising into stratocumulus as observed during the first 'lagrangian' experiment of astex. Quarterly Journal of the Royal Meteorological Society, 122(535):1597-1623.

de Roode, S. R., Sandu, I., van der Dussen, J. J., Ackerman, A. S., Blossey, P., Jarecka, D., Lock, A., Siebesma, A. P., and Stevens, B. (2016). Large-eddy simulations of euclipse-gass lagrangian stratocumulus-to-cumulus transitions: Mean state, turbulence, and decoupling. Journal of the Atmospheric Sciences, 73(6):2485-2508.

Dearden, C., Hill, A., Coe, H., and Choularton, T. (2018). The role of droplet sedimentation in the evolution of low-level clouds over southern west africa. Atmospheric Chemistry and Physics, 18(19):14253-14269.

Deardorff, J. W. (1980). Stratocumulus-capped mixed layers derived from a threedimensional model. Boundary-Layer Meteorology, 18(4):495-527.

Deng, A., Gaudet, B., Dudhia, J., and Alapaty, K. (2014). Implementation and evaluation of a new shallow convection scheme in WRF. In 26th Conf. on Weather Analysis and Forecasting/22nd Conf. on Numerical Weather Prediction.

Deng, A., Seaman, N. L., and Kain, J. S. (2003). A shallow-convection parameterization for mesoscale models. Part I: Submodel description and preliminary applications. Journal of the Atmospheric Sciences, 60(1):34-56.

Derrien, S., Bezombes, Y., Bret, G., Gabella, O., Jarnot, C., Medina, P., Piques, E., Delon, C., Dione, C., Campistron, B., Durand, P., Jambert, C., Lohou, F., Lothon, M., Pacifico, F., and Meyerfeld, Y. (2016). Dacciwa field campaign, savè super-site, ups instrumentation. SEDOO OMP.

Dione, C., Lohou, F., Lothon, M., Adler, B., Babić, K., Kalthoff, N., Pedruzo-Bagazgoitia, X., Bezombes, Y., and Gabella, O. (2019). Low-level stratiform clouds and dynamical features observed within the southern west african monsoon. Atmospheric Chemistry and Physics, 19(13):8979-8997.

Dudhia, J. (2014). A history of mesoscale model development. Asia-Pacific Journal of Atmospheric Sciences, 50(1):121-131.

Duynkerke, P. G. (1998). Dynamics of cloudy boundary layers. In Holtslag, A. and Duynkerke, P. G., editors, Clear and Cloudy Boundary Layers: Proceedings of the 
Colloquium "Clear and Cloudy Boundary Layers". Amsterdam, 26-29 August 1997, chapter 9, pages 199-218. Royal Netherlands Academy of Arts and Sciences.

Duynkerke, P. G., de Roode, S. R., van Zanten, M. C., Calvo, J., Cuxart, J., Cheinet, S., Chlond, A., Grenier, H., Jonker, P. J., Köhler, M., Lenderink, G., Lewellen, D., Lappen, C.-l., Lock, A. P., Moeng, C.-h., Müller, F., Olmeda, D., Piriou, J.m., Sánchez, E., and Sednev, I. (2004). Observations and numerical simulations of the diurnal cycle of the eurocs stratocumulus case. Quarterly Journal of the Royal Meteorological Society, 130(604):3269-3296.

Eastman, R., Warren, S. G., , and Hahn, C. J. (2014). Climatic atlas of cloudsover land and ocean [available online at : https://atmos.washington.edu/cloudmap/].

Eastman, R. and Warren, S. G. (2014). Diurnal cycles of cumulus, cumulonimbus, stratus, stratocumulus, and fog from surface observations over land and ocean. Journal of Climate, 27(6):2386-2404.

Ek, M. and Mahrt, L. (1994). Daytime evolution of relative humidity at the boundary layer top. Monthly Weather Review, 122(12):2709-2721.

Ek, M. B. and Holtslag, A. A. M. (2004). Influence of soil moisture on boundary layer cloud development. Journal of Hydrometeorology, 5(1):86-99.

Esau, I. and Lyons, T. (2002). Effect of sharp vegetation boundary on the convective atmospheric boundary layer. Agricultural and Forest Meteorology, 114(1-2):3-13.

Finnigan, J. J., Shaw, R. H., and Patton, E. G. (2009). Turbulence structure above a vegetation canopy. Journal of Fluid Mechanics, 637:387-424.

Flamant, C., Knippertz, P., Fink, A. H., Akpo, A., Brooks, B., Chiu, C. J., Coe, H., Danuor, S., Evans, M., Jegede, O., Kalthoff, N., Konaré, A., Liousse, C., Lohou, F., Mari, C., Schlager, H., Schwarzenboeck, A., Adler, B., Amekudzi, L., Aryee, J., Ayoola, M., Batenburg, A. M., Bessardon, G., Borrmann, S., Brito, J., Bower, K., Burnet, F., Catoire, V., Colomb, A., Denjean, C., Fosu-Amankwah, K., Hill, P. G., Lee, J., Lothon, M., Maranan, M., Marsham, J., Meynadier, R., Ngamini, J.-B., Rosenberg, P., Sauer, D., Smith, V., Stratmann, G., Taylor, J. W., Voigt, C., and Yoboué, V. (2018). The dynamics-aerosol-chemistry-cloud interactions in west africa field campaign: Overview and research highlights. Bulletin of the American Meteorological Society, 99(1):83-104.

Fowler, L. D., Skamarock, W. C., Grell, G. A., Freitas, S. R., and Duda, M. G. (2016). Analyzing the Grell-Freitas convection scheme from hydrostatic to nonhydrostatic scales within a global model. Monthly Weather Review, 144(6):2285-2306.

Freedman, J. M., Fitzjarrald, D. R., Moore, K. E., and Sakai, R. K. (2001). Boundary layer clouds and vegetation-atmosphere feedbacks. Journal of Climate, 14(2):180197. 
Freitas, S. R., Panetta, J., Longo, K. M., Rodrigues, L. F., Moreira, D. S., Rosário, N. E., Silva Dias, P. L., Silva Dias, M. A. F., Souza, E. P., Freitas, E. D., Longo, M., Frassoni, A., Fazenda, A. L., Santos e Silva, C. M., Pavani, C. A. B., Eiras, D., França, D. A., Massaru, D., Silva, F. B., Santos, F. C., Pereira, G., Camponogara, G., Ferrada, G. A., Campos Velho, H. F., Menezes, I., Freire, J. L., Alonso, M. F., Gácita, M. S., Zarzur, M., Fonseca, R. M., Lima, R. S., Siqueira, R. A., Braz, R., Tomita, S., Oliveira, V., and Martins, L. D. (2017). The brazilian developments on the Regional Atmospheric Modeling System (BRAMS 5.2): an integrated environmental model tuned for tropical areas. Geoscientific Model Development, 10(1):189-222.

Garcia-Carreras, L., Parker, D. J., and Marsham, J. H. (2011). What is the mechanism for the modification of convective cloud distributions by land surface-induced flows? Journal of the Atmospheric Sciences, 68(3):619-634.

Garcia-Carreras, L., Parker, D. J., Taylor, C. M., Reeves, C. E., and Murphy, J. G. (2010). Impact of mesoscale vegetation heterogeneities on the dynamical and thermodynamic properties of the planetary boundary layer. Journal of Geophysical Research: Atmospheres, 115(D3):n/a-n/a. D03102.

Garratt, J. R. (1992). The atmospheric boundary layer / J. R. Garratt. Cambridge University Press Cambridge ; New York.

Ghate, V. P., Albrecht, B. A., Miller, M. A., Brewer, A., and Fairall, C. W. (2014). Turbulence and radiation in stratocumulus-topped marine boundary layers: A case study from vocals-rex. Journal of Applied Meteorology and Climatology, 53(1):117135.

Ghonima, M. S., Heus, T., Norris, J. R., and Kleissl, J. (2016). Factors controlling stratocumulus cloud lifetime over coastal land. Journal of the Atmospheric Sciences, 73(8):2961-2983.

Golaz, J.-C., Jiang, H., and Cotton, W. R. (2001). A large-eddy simulation study of cumulus clouds over land and sensitivity to soil moisture. Atmospheric Research, 59-60:373 - 392. 13th International Conference on Clouds and Precipitation.

Goudriaan, J. (1977). Crop micrometeorology: a simulation study. Pudoc, Center for Agricultural Publishing and Documentation.

Goudriaan, J. (1986). A simple and fast numerical method for the computation of daily totals of crop photosynthesis. Agricultural and Forest Meteorology, 38(1):249 - 254.

Goudriaan, J. (1988). The bare bones of leaf-angle distribution in radiation models for canopy photosynthesis and energy exchange. Agricultural and Forest Meteorology, 43(2):155-169.

Grabowski, W. W. (2014). Extracting microphysical impacts in large-eddy simulations of shallow convection. Journal of the Atmospheric Sciences, 71(12):4493-4499. 
Grell, G. A. (1993). Prognostic evaluation of assumptions used by cumulus parameterizations. Monthly Weather Review, 121(3):764-787.

Grell, G. A. and Devenyi, D. (2002). A generalized approach to parameterizing convection combining ensemble and data assimilation techniques. Geophysical Research Letters, 29(14):38-1-38-4.

Grell, G. A. and Freitas, S. R. (2014). A scale and aerosol aware stochastic convective parameterization for weather and air quality modeling. Atmospheric Chemistry and Physics, 14(10):5233-5250.

Gronemeier, T., Kanani-Sühring, F., and Raasch, S. (2016). Do shallow cumulus clouds have the potential to trigger secondary circulations via shading? Boundary-Layer Meteorology, pages 1-27.

Gu, L., Baldocchi, D., Verma, S. B., Black, T. A., Vesala, T., Falge, E. M., and Dowty, P. R. (2002). Advantages of diffuse radiation for terrestrial ecosystem productivity. Journal of Geophysical Research: Atmospheres, 107(5-6):2-1 - 2-21.

Gu, L., Baldocchi, D. D., Wofsy, S. C., Munger, J. W., Michalsky, J. J., Urbanski, S. P., and Boden, T. A. (2003). Response of a deciduous forest to the mount pinatubo eruption: Enhanced photosynthesis. Science, 299(5615):2035-2038.

Guenther, A. (2013). Upscaling Biogenic Volatile Compound Emissions from Leaves to Landscapes, pages 391-414. Springer Netherlands, Dordrecht.

Guichard, F., Petch, J. C., Redelsperger, J., Bechtold, P., Chaboureau, J., Cheinet, S., Grabowski, W., Grenier, H., Jones, C. G., Kohler, M., Piriou, J., Tailleux, R., and Tomasini, M. (2004). Modelling the diurnal cycle of deep precipitating convection over land with cloud-resolving models and single-column models. Quarterly Journal of the Royal Meteorological Society, 130 C(604):3139-3172.

Handwerker, J., Scheer, S., and Gamer, T. (2016). Dacciwa field campaign, savè supersite, cloud and precipitation. SEDOO OMP.

Hannak, L., Knippertz, P., Fink, A. H., Kniffka, A., and Pante, G. (2017). Why do global climate models struggle to represent low-level clouds in the west african summer monsoon? Journal of Climate, 30(5):1665-1687.

Hartmann, D. L., Ockert-Bell, M. E., and Michelsen, M. L. (1992). The effect of cloud type on earth's energy balance: Global analysis. Journal of Climate, 5(11):1281-1304.

Heus, T. and Jonker, H. J. J. (2008). Subsiding shells around shallow cumulus clouds. Journal of the Atmospheric Sciences, 65(3):1003-1018.

Heus, T., van Heerwaarden, C. C., Jonker, H. J. J., Pier Siebesma, A., Axelsen, S., van den Dries, K., Geoffroy, O., Moene, A. F., Pino, D., de Roode, S. R., and Vilà-Guerau de Arellano, J. (2010). Formulation of the Dutch Atmospheric LargeEddy Simulation (DALES) and overview of its applications. Geoscientific Model 
Development, 3(2):415-444.

Hill, P. G., Allan, R. P., Chiu, J. C., Bodas-Salcedo, A., and Knippertz, P. (2018). Quantifying the contribution of different cloud types to the radiation budget in southern west africa. Journal of Climate, 31(13):5273-5291.

Hong, S.-Y. and Dudhia, J. (2012). Next-generation numerical weather prediction: Bridging parameterization, explicit clouds, and large eddies. Bulletin of the American Meteorological Society, 93(1):ES6-ES9.

Hong, S.-Y., Juang, H.-M. H., and Zhao, Q. (1998). Implementation of prognostic cloud scheme for a regional spectral model. Monthly Weather Review, 126(10):2621-2639.

Horn, G. L., Ouwersloot, H. G., Vilà-Guerau de Arellano, J., and Sikma, M. (2015). Cloud shading effects on characteristic boundary-layer length scales. Boundary-Layer Meteorology, 157(2):237-263.

Huang, H.-Y. and Margulis, S. A. (2010). Evaluation of a fully coupled large-eddy simulation-land surface model and its diagnosis of land-atmosphere feedbacks. Water Resources Research, 46(6):n/a-n/a. W06512.

Iacono, M. J., Delamere, J. S., Mlawer, E. J., Shephard, M. W., Clough, S. A., and Collins, W. D. (2008). Radiative forcing by long-lived greenhouse gases: Calculations with the AER radiative transfer models. Journal of Geophysical Research: Atmospheres, 113(D13).

Jacobs, C., van den Hurk, B., and de Bruin, H. (1996). Stomatal behaviour and photosynthetic rate of unstressed grapevines in semi-arid conditions. Agricultural and Forest Meteorology, 80(2):111 - 134 .

Jacobs, C. M. J. (1994). Direct impact of atmospheric CO2 enrichment on regional transpiration. PhD thesis, Wageningen Agricultural University, The Netherlands.

Jacobs, C. M. J. and De Bruin, H. A. R. (1992). The sensitivity of regional transpiration to land-surface characteristics: Significance of feedback. Journal of Climate, 5(7):683698.

Jacobs, C. M. J. and de Bruin, H. A. R. (1997). Predicting regional transpiration at elevated atmospheric CO2: Influence of the PBL-vegetation interaction. Journal of Applied Meteorology, 36(12):1663-1675.

Jakub, F. (2016). On the impact of three dimensional radiative transfer on cloud evolution. $\mathrm{PhD}$ thesis, Ludwig-Maximilians-Universität München.

Jakub, F. and Mayer, B. (2015). A three-dimensional parallel radiative transfer model for atmospheric heating rates for use in cloud resolving models - the tenstream solver. Journal of Quantitative Spectroscopy and Radiative Transfer, 163:63 - 71.

Jakub, F. and Mayer, B. (2017). The role of 1-D and 3-D radiative heating in the organization of shallow cumulus convection and the formation of cloud streets. Atmospheric 
Chemistry \& Physics, 17:13317-13327.

Jimenez, P. A., Alessandrini, S., Haupt, S. E., Deng, A., Kosovic, B., Lee, J. A., and Monache, L. D. (2016a). The role of unresolved clouds on short-range global horizontal irradiance predictability. Monthly Weather Review, 144(9):3099-3107.

Jimenez, P. A., Hacker, J. P., Dudhia, J., Haupt, S. E., Ruiz-Arias, J. A., Gueymard, C. A., Thompson, G., Eidhammer, T., and Deng, A. (2016b). WRF-Solar: Description and clear-sky assessment of an augmented NWP model for solar power prediction. Bulletin of the American Meteorological Society, 97(7):1249-1264.

Joseph, J. H., Wiscombe, W. J., and Weinman, J. A. (1976). The delta-eddington approximation for radiative flux transfer. Journal of the Atmospheric Sciences, 33(12):24522459.

Kain, J. S. and Fritsch, J. M. (1990). A one-dimensional entraining/detraining plume model and its application in convective parameterization. Journal of the Atmospheric Sciences, 47(23):2784-2802.

Kalthoff, N., Lohou, F., Brooks, B., Jegede, G., Adler, B., Babić, K., Dione, C., Ajao, A., Amekudzi, L. K., Aryee, J. N. A., Ayoola, M., Bessardon, G., Danuor, S. K., Handwerker, J., Kohler, M., Lothon, M., Pedruzo-Bagazgoitia, X., Smith, V., Sunmonu, L., Wieser, A., Fink, A. H., and Knippertz, P. (2018). An overview of the diurnal cycle of the atmospheric boundary layer during the west african monsoon season: results from the 2016 observational campaign. Atmospheric Chemistry and Physics, 18(4):2913-2928.

Kanniah, K. D., Beringer, J., North, P., and Hutley, L. (2012). Control of atmospheric particles on diffuse radiation and terrestrial plant productivity: A review. Progress in Physical Geography, 36(2):209-237.

Katul, G. G., Oren, R., Manzoni, S., Higgins, C., and Parlange, M. B. (2012). Evapotranspiration: A process driving mass transport and energy exchange in the soil-plantatmosphere-climate system. Reviews of Geophysics, 50(3).

Kazil, J., Feingold, G., and Yamaguchi, T. (2016). Wind speed response of marine nonprecipitating stratocumulus clouds over a diurnal cycle in cloud-system resolving simulations. Atmospheric Chemistry and Physics, 16(9):5811-5839.

Keenan, T., Baker, I., Barr, A., Ciais, P., Davis, K., Dietze, M., Dragoni, D., Gough, C. M., Grant, R., Hollinger, D., Hufkens, K., Poulter, B., McCaughey, H., Raczka, B., Ryu, Y., Schaefer, K., Tian, H., Verbeeck, H., Zhao, M., and Richardson, A. D. (2012). Terrestrial biosphere model performance for inter-annual variability of landatmosphere co2 exchange. Global Change Biology, 18(6):1971-1987.

Khairoutdinov, M. and Kogan, Y. (2000). A new cloud physics parameterization in a large-eddy simulation model of marine stratocumulus. Monthly Weather Review, $128(1): 229-243$. 
Kivalov, S. N. and Fitzjarrald, D. R. (2018). Quantifying and modelling the effect of cloud shadows on the surface irradiance at tropical and midlatitude forests. Boundary-Layer Meteorology, 166(2):165-198.

Kivalov, S. N. and Fitzjarrald, D. R. (2019). Observing the whole-canopy short-term dynamic response to natural step changes in incident light: Characteristics of tropical and temperate forests. Boundary-Layer Meteorology, 173(1):1-52.

Klinger, C., Mayer, B., Jakub, F., Zinner, T., Park, S.-B., and Gentine, P. (2017). Effects of 3-D thermal radiation on the development of a shallow cumulus cloud field. Atmospheric Chemistry and Physics, 17(8):5477-5500.

Knippertz, P., Coe, H., Chiu, J. C., Evans, M. J., Fink, A. H., Kalthoff, N., Liousse, C., Mari, C., Allan, R. P., Brooks, B., Danour, S., Flamant, C., Jegede, O. O., Lohou, F., and Marsham, J. H. (2015). The dacciwa project: Dynamics-aerosol-chemistry-cloud interactions in west africa. Bulletin of the American Meteorological Society, 96(9):1451-1460.

Knippertz, P., Fink, A. H., Schuster, R., Trentmann, J., Schrage, J. M., and Yorke, C. (2011). Ultra-low clouds over the southern west african monsoon region. Geophysical Research Letters, 38(21).

Knohl, A. and Baldocchi, D. D. (2008). Effects of diffuse radiation on canopy gas exchange processes in a forest ecosystem. Journal of Geophysical Research: Biogeosciences, 113(G2):n/a-n/a. G02023.

Kobayashi, H., Baldocchi, D. D., Ryu, Y., Chen, Q., Ma, S., Osuna, J. L., and Ustin, S. L. (2012). Modeling energy and carbon fluxes in a heterogeneous oak woodland: A three-dimensional approach. Agricultural and Forest Meteorology, 152:83 - 100.

Kohler, M., Kalthoff, N., Seringer, J., and Kraut, S. (2016). Dacciwa field campaign, save super-site, surface measurements. SEDOO OMP.

Laffineur, Q., Aubinet, M., Schoon, N., Amelynck, C., Muller, J., Dewulf, J., Steppe, K., and Heinesch, B. (2013). Impact of diffuse light on isoprene and monoterpene emissions from a mixed temperate forest. Atmospheric Environment, 74:385 - 392.

Lenderink, G., Siebesma, A. P., Cheinet, S., Irons, S., Jones, C. G., Marquet, P., üLLER, F. M., Olmeda, D., Calvo, J., Sánchez, E., and Soares, P. M. M. (2004). The diurnal cycle of shallow cumulus clouds over land: A single-column model intercomparison study. Quarterly Journal of the Royal Meteorological Society, 130(604):3339-3364.

Lenschow, D. H., Lothon, M., Mayor, S. D., Sullivan, P. P., and Canut, G. (2012). A comparison of higher-order vertical velocity moments in the convective boundary layer from lidar with in situ measurements and large-eddy simulation. BoundaryLayer Meteorology, 143(1):107-123.

Lewellen, D. C. and Lewellen, W. S. (2002). Entrainment and decoupling relations for 
cloudy boundary layers. Journal of the Atmospheric Sciences, 59(20):2966-2986.

Lilly, D. K. (1968). Models of cloud-topped mixed layers under a strong inversion. Quarterly Journal of the Royal Meteorological Society, 94(401):292-309.

Liou, K.-N. (2002). An introduction to atmospheric radiation, volume 84. Academic press.

Liu, C., Fedorovich, E., and Huang, J. (2018). Revisiting entrainment relationships for shear-free and sheared convective boundary layers through large-eddy simulations. Quarterly Journal of the Royal Meteorological Society, 144(716):2182-2195.

Lobos-Roco, F., Vilà-Guerau de Arellano, J., and Pedruzo-Bagazgoitia, X. (2018). Characterizing the influence of the marine stratocumulus cloud on the land fog at the atacama desert. Atmospheric Research, 214:109 - 120.

Lohou, F., Kalthoff, N., Adler, B., Babić, K., Dione, C., Lothon, M., Pedruzo-Bagazgoitia, X., and Zouzoua, M. (2019). Conceptual model of diurnal cycle of stratiform low-level clouds over southern west africa. Atmospheric Chemistry and Physics Discussions, 2019:1-25.

Lohou, F. and Patton, E. G. (2014). Surface energy balance and buoyancy response to shallow cumulus shading. Journal of the Atmospheric Sciences, 71(2):665-682.

Lorenc, A. C., Barker, D., Bell, R. S., Macpherson, B., and Maycock, A. J. (1996). On the use of radiosonde humidity observations in mid-latitude nwp. Meteorology and Atmospheric Physics, 60(1):3-17.

Lu, C., Niu, S., Liu, Y., and Vogelmann, A. M. (2013). Empirical relationship between entrainment rate and microphysics in cumulus clouds. Geophysical Research Letters, 40(10):2333-2338.

Madronich, S. (1987). Photodissociation in the atmosphere: 1. actinic flux and the effects of ground reflections and clouds. Journal of Geophysical Research: Atmospheres, 92(D8):9740-9752.

Mauder, M., Cuntz, M., Drüe, C., Graf, A., Rebmann, C., Schmid, H. P., Schmidt, M., and Steinbrecher, R. (2013). A strategy for quality and uncertainty assessment of long-term eddy-covariance measurements. Agr. Forest Meteorol., 169:122-135.

McFarlane, S. A. and Grabowski, W. W. (2007). Optical properties of shallow tropical cumuli derived from arm ground-based remote sensing. Geophysical Research Letters, 34(6):n/a-n/a. L06808.

McMichael, L. A., Mechem, D. B., Wang, S., Wang, Q., Kogan, Y. L., and Teixeira, J. (2019). Assessing the mechanisms governing the daytime evolution of marine stratocumulus using large-eddy simulation. Quarterly Journal of the Royal Meteorological Society, 145(719):845-866.

Mechem, D. B., Kogan, Y. L., and Schultz, D. M. (2010). Large-eddy simulation of post-cold-frontal continental stratocumulus. Journal of the Atmospheric Sciences, 
67(12):3835-3853.

Mellado, J. P. (2017). Cloud-top entrainment in stratocumulus clouds. Annual Review of Fluid Mechanics, 49(1):145-169.

Mercado, L. M., Bellouin, N., Sitch, S., Boucher, O., Huntingford, C., Wild, M., and Cox, P. M. (2009). Impact of changes in diffuse radiation on the global land carbon sink. Nature, 458(7241):1014-1017.

Min, Q. (2005). Impacts of aerosols and clouds on forest-atmosphere carbon exchange. Journal of Geophysical Research: Atmospheres, 110(D6):n/a-n/a. D06203.

Min, Q. and Wang, S. (2008). Clouds modulate terrestrial carbon uptake in a midlatitude hardwood forest. Geophysical Research Letters, 35(2):n/a-n/a. L02406.

Miralles, D. G., Teuling, A. J., van Heerwaarden, C. C., and Vilà-Guerau de Arellano, J. (2014). Mega-heatwave temperatures due to combined soil desiccation and atmospheric heat accumulation. Nature Geoscience, 7:345 EP -.

Moene, A. F. and Van Dam, J. C. (2014). Transport in the atmosphere-vegetation-soil continuum. Cambridge University Press.

Moeng, C.-H. (1998). large eddy simulation of atmospheric boundary layers. In Holtslag, A. and Duynkerke, P. G., editors, Clear and Cloudy Boundary Layers: Proceedings of the Colloquium "Clear and Cloudy Boundary Layers". Amsterdam, 26-29 August 1997, chapter 3, pages 67-83. Royal Netherlands Academy of Arts and Sciences.

Morrison, H., de Boer, G., Feingold, G., Harrington, J., Shupe, M. D., and Sulia, K. (2012). Resilience of persistent Arctic mixed-phase clouds. Nature Geoscience, 5:1117.

Nakanishi, M. and Niino, H. (2006). An improved Mellor-Yamada Level-3 Model: Its numerical stability and application to a regional prediction of advection fog. BoundaryLayer Meteorology, 119(2):397-407.

Neggers, R., Stevens, B., and Neelin, J. D. (2006). A simple equilibrium model for shallow-cumulus-topped mixed layers. Theoretical and Computational Fluid Dynamics, 20(5):305-322.

Neggers, R. A. J., Jonker, H. J. J., and Siebesma, A. P. (2003). Size statistics of cumulus cloud populations in large-eddy simulations. Journal of the Atmospheric Sciences, 60(8):1060-1074.

Nieuwstadt, F. T. M. and Brost, R. A. (1986). The decay of convective turbulence. Journal of the Atmospheric Sciences, 43(6):532-546.

Niu, G., Yang, Z., Mitchell, K. E., Chen, F., Ek, M. B., Barlage, M., Kumar, A., Manning, K., Niyogi, D., Rosero, E., Tewari, M., and Xia, Y. (2011). The community Noah land surface model with multiparameterization options (Noah-MP): 1. model description and evaluation with local scale measurements. Journal of Geophysical Research: 
Atmospheres, 116(D12).

Norman, J. (1979). Modeling the complete crop canopy. Modification of the Aerial Environment of Crops, pages 249-280.

Oliphant, A., Dragoni, D., Deng, B., Grimmond, C., Schmid, H.-P., and Scott, S. (2011). The role of sky conditions on gross primary production in a mixed deciduous forest. Agricultural and Forest Meteorology, 151(7):781 - 791.

Oliveira, P. J. C., Davin, E. L., Levis, S., and Seneviratne, S. I. (2011). Vegetationmediated impacts of trends in global radiation on land hydrology: a global sensitivity study. Global Change Biology, 17(11):3453-3467.

Ouwersloot, H. G., de Arellano, J. V., H. van Stratum, B. J., Krol, M. C., and Lelieveld, J. (2013). Quantifying the transport of subcloud layer reactants by shallow cumulus clouds over the amazon. Journal of Geophysical Research: Atmospheres, 118(23):13,041-13,059.

Ouwersloot, H. G., Moene, A. F., Attema, J. J., and de Arellano, J. V.-G. (2016). Largeeddy simulation comparison of neutral flow over a canopy: Sensitivities to physical and numerical conditions, and similarity to other representations. Boundary-Layer Meteorology, pages 1-19.

Ouwersloot, H. G., Vilà-Guerau de Arellano, J., van Heerwaarden, C. C., Ganzeveld, L. N., Krol, M. C., and Lelieveld, J. (2011). On the segregation of chemical species in a clear boundary layer over heterogeneous land surfaces. Atmospheric Chemistry and Physics, 11(20):10681-10704.

Patton, E. G., Sullivan, P. P., and Moeng, C.-H. (2005). The influence of idealized heterogeneity on wet and dry planetary boundary layers coupled to the land surface. Journal of the Atmospheric Sciences, 62(7):2078-2097.

Pedro, H. T. and Coimbra, C. F. (2012). Assessment of forecasting techniques for solar power production with no exogenous inputs. Solar Energy, 86(7):2017 - 2028.

Pedruzo-Bagazgoitia, X., Jiménez, P. A., Dudhia, J., and Vilà-Guerau de Arellano, J. (2019a). Shallow cumulus representation and its interaction with radiation and surface at the convection gray zone. Monthly Weather Review, 147(7):2467-2483.

Pedruzo-Bagazgoitia, X., de Roode, S. R., Adler, B., Babić, K., Dione, C., Kalthoff, N., Lohou, F., Lothon, M., and Vilà-Guerau de Arellano, J. (2019b). The diurnal stratocumulus-to-cumulus transition over land. Atmospheric Chemistry and Physics Discussions, 2019:1-30.

Pedruzo-Bagazgoitia, X., Ouwersloot, H. G., Sikma, M., van Heerwaarden, C. C., Jacobs, C. M. J., and Vilà-Guerau de Arellano, J. (2017). Direct and diffuse radiation in the shallow cumulus-vegetation system: Enhanced and decreased evapotranspiration regimes. Journal of Hydrometeorology, 18(6):1731-1748. 
Pedruzo-Bagazgoitia, X., Veerman, M., Jakub, F., van Heerwaarden, C. C., and VilàGuerau de Arellano, J. (In preparation). Emergent features on cloud convection by coupling $3 \mathrm{~d}$ radiation and surface on large eddy simulation.

Pino, D., Vilà-Guerau de Arellano, J., and Duynkerke, P. G. (2003). The contribution of shear to the evolution of a convective boundary layer. Journal of the Atmospheric Sciences, 60(16):1913-1926.

Powers, J. G., Klemp, J. B., Skamarock, W. C., Davis, C. A., Dudhia, J., Gill, D. O., Coen, J. L., Gochis, D. J., Ahmadov, R., Peckham, S. E., Grell, G. A., Michalakes, J., Trahan, S., Benjamin, S. G., Alexander, C. R., Dimego, G. J., Wang, W., Schwartz, C. S., Romine, G. S., Liu, Z., Snyder, C., Chen, F., Barlage, M. J., Yu, W., and Duda, M. G. (2017). The weather research and forecasting model: Overview, system efforts, and future directions. Bulletin of the American Meteorological Society, 98(8):17171737.

Priestley, C. H. B. and Taylor, R. J. (1972). On the assessment of surface heat flux and evaporation using large-scale parameters. Monthly Weather Review, 100(2):81-92.

Rieck, M., Hohenegger, C., and van Heerwaarden, C. C. (2014). The influence of land surface heterogeneities on cloud size development. Monthly Weather Review, 142(10):3830-3846.

Ronda, R. J., de Bruin, H. A. R., and Holtslag, A. A. M. (2001). Representation of the canopy conductance in modeling the surface energy budget for low vegetation. Journal of Applied Meteorology, 40(8):1431-1444.

Sandu, I. and Stevens, B. (2011). On the factors modulating the stratocumulus to cumulus transitions. Journal of the Atmospheric Sciences, 68(9):1865-1881.

Schalkwijk, J., Jonker, H. J. J., Siebesma, A. P., and Bosveld, F. C. (2015). A year-long large-eddy simulation of the weather over cabauw: An overview. Monthly Weather Review, 143(3):828-844.

Schneider, T., Kaul, C. M., and Pressel, K. G. (2019). Possible climate transitions from breakup of stratocumulus decks under greenhouse warming. Nature Geoscience, 12(3):163-167.

Schumann, U., Dörnbrack, A., and Mayer, B. (2002). Cloud-shadow effects on the structure of the convective boundary layer. Meteorologische Zeitschrift, 11(4):285-294.

Schwartz, S. E., Huang, D., and Vladutescu, D. V. (2017). High-resolution photography of clouds from the surface: Retrieval of optical depth of thin clouds down to centimeter scales. Journal of Geophysical Research: Atmospheres, pages n/a-n/a. 2016JD025384.

Shettle, E. P. and Weinman, J. A. (1970). The transfer of solar irradiance through inhomogeneous turbid atmospheres evaluated by eddington's approximation. Journal 
of the Atmospheric Sciences, 27(7):1048-1055.

Siebesma, A. P., Bretherton, C. S., Brown, A., Chlond, A., Cuxart, J., Duynkerke, P. G., Jiang, H., Khairoutdinov, M., Lewellen, D., Moeng, C.-H., et al. (2003). A large eddy simulation intercomparison study of shallow cumulus convection. Journal of the Atmospheric Sciences, 60(10):1201-1219.

Sikma, M. and Ouwersloot, H. G. (2015). Parameterizations for convective transport in various cloud-topped boundary layers. Atmospheric Chemistry and Physics, 15(18):10399-10410.

Sikma, M., Ouwersloot, H. G., Pedruzo-Bagazgoitia, X., van Heerwaarden, C. C., and Vilà-Guerau de Arellano, J. (2018). Interactions between vegetation, atmospheric turbulence and clouds under a wide range of background wind conditions. Agricultural and Forest Meteorology, 255:31 - 43. Honoring W.J. Massman's Discoveries: Bringing Physics to Agriculture.

Sikma, M. and Vilà-Guerau de Arellano, J. (2019). Substantial reductions in cloud cover and moisture transport by dynamic plant responses. Geophysical Research Letters, 46(3):1870-1878.

Sikma, M., Vilà-Guerau de Arellano, J., Pedruzo-Bagazgoitia, X., Heusinkveld, B., Anten, N., and Evers, J. (2019). Impact of future warming and enhanced CO2 on the vegetation-cloud interaction. Journal of Geophysical Research: Atmospheres. In press.

Skamarock, W. C., Klemp, J. B., Dudhia, J., Gill, D. O., Barker, D. M., Duda, M. G., Huang, X.-Y., Wang, W., and Powers, J. G. (2008). A description of the Advanced Research WRF Version 3. NCAR Tech. Note NCAR/TN-475+STR, 113 pp.

Slawinska, J., Grabowski, W. W., Pawlowska, H., and Wyszogrodzki, A. A. (2008). Optical properties of shallow convective clouds diagnosed from a bulk-microphysics largeeddy simulation. Journal of Climate, 21(7):1639-1647.

Smagorinsky, J. (1963). General circulation experiments with the primitive equations. Monthly Weather Review, 91(3):99-164.

Sommeria, G. (1976). Three-dimensional simulation of turbulent processes in an undisturbed trade wind boundary layer. Journal of the Atmospheric Sciences, 33(2):216241.

Spitters, C. (1986). Separating the diffuse and direct component of global radiation and its implications for modeling canopy photosynthesis part ii. calculation of canopy photosynthesis. Agricultural and Forest Meteorology, 38(1):231 - 242.

Spitters, C., Toussaint, H., and Goudriaan, J. (1986). Separating the diffuse and direct component of global radiation and its implications for modeling canopy photosynthesis part i. components of incoming radiation. Agricultural and Forest Meteorology, 
$38(1): 217-229$.

Stensrud, D. (2011). Parameterization schemes: Keys to understanding numerical weather prediction models, volume 9780521865401. Cambridge University Press, United Kingdom.

Stephens, G. L. (1984). The parameterization of radiation for numerical weather prediction and climate models. Monthly Weather Review, 112(4):826-867.

Stevens, B. (2000). Cloud transitions and decoupling in shear-free stratocumulus-topped boundary layers. Geophysical Research Letters, 27(16):2557-2560.

Stevens, B., Lenschow, D. H., Faloona, I., Moeng, C.-H., Lilly, D. K., Blomquist, B., Vali, G., Bandy, A., Campos, T., Gerber, H., Haimov, S., Morley, B., and Thornton, D. (2003). On entrainment rates in nocturnal marine stratocumulus. Quarterly Journal of the Royal Meteorological Society, 129(595):3469-3493.

Stevens, B., Moeng, C.-H., Ackerman, A. S., Bretherton, C. S., Chlond, A., de Roode, S., Edwards, J., Golaz, J.-C., Jiang, H., Khairoutdinov, M., Kirkpatrick, M. P., Lewellen, D. C., Lock, A., Müller, F., Stevens, D. E., Whelan, E., and Zhu, P. (2005). Evaluation of large-eddy simulations via observations of nocturnal marine stratocumulus. Monthly Weather Review, 133(6):1443-1462.

Stull, R. B. (1988). An introduction to boundary layer meteorology, volume 13. Springer Science \& Business Media.

Taylor, J. W., Haslett, S. L., Bower, K., Flynn, M., Crawford, I., Dorsey, J., Choularton, T., Connolly, P. J., Hahn, V., Voigt, C., Sauer, D., Dupuy, R., Brito, J., Schwarzenboeck, A., Bourriane, T., Denjean, C., Rosenberg, P., Flamant, C., Lee, J. D., Vaughan, A. R., Hill, P. G., Brooks, B., Catoire, V., Knippertz, P., and Coe, H. (2019). Aerosol influences on low-level clouds in the west african monsoon. Atmospheric Chemistry and Physics Discussions, 2019:1-45.

Thompson, G., Field, P. R., Rasmussen, R. M., and Hall, W. D. (2008). Explicit forecasts of winter precipitation using an improved bulk microphysics scheme. Part II: Implementation of a new snow parameterization. Monthly Weather Review, 136(12):50955115 .

Trenberth, K. E., Fasullo, J. T., and Kiehl, J. (2009). Earth's global energy budget. Bulletin of the American Meteorological Society, 90(3):311-324.

Turton, J. D. and Nicholls, S. (1987). A study of the diurnal variation of stratocumulus using a multiple mixed layer model. Quarterly Journal of the Royal Meteorological Society, 113(477):969-1009.

Urban, O., Klem, K., Ač, A., Havránková, K., Holišová, P., Navrátil, M., Zitová, M., Kozlová, K., Pokorný, R., Šprtová, M., Tomášková, I., Špunda, V., and Grace, J. (2012). Impact of clear and cloudy sky conditions on the vertical distribution of 
photosynthetic co2 uptake within a spruce canopy. Functional Ecology, 26(1):46-55.

van der Dussen, J. J., de Roode, S. R., Ackerman, A. S., Blossey, P. N., Bretherton, C. S., Kurowski, M. J., Lock, A. P., Neggers, R. A. J., Sandu, I., and Siebesma, A. P. (2013). The gass/euclipse model intercomparison of the stratocumulus transition as observed during astex: Les results. Journal of Advances in Modeling Earth Systems, 5(3):483-499.

van der Dussen, J. J., de Roode, S. R., Dal Gesso, S., and Siebesma, A. P. (2015). An les model study of the influence of the free tropospheric thermodynamic conditions on the stratocumulus response to a climate perturbation. Journal of Advances in Modeling Earth Systems, 7(2):670-691.

van der Dussen, J. J., de Roode, S. R., and Siebesma, A. P. (2014). Factors controlling rapid stratocumulus cloud thinning. Journal of the Atmospheric Sciences, 71(2):655664.

van der Dussen, J. J., de Roode, S. R., and Siebesma, A. P. (2016). How large-scale subsidence affects stratocumulus transitions. Atmospheric Chemistry and Physics, 16(2):691-701.

van der Linden, R., Fink, A. H., and Redl, R. (2015). Satellite-based climatology of lowlevel continental clouds in southern west africa during the summer monsoon season. Journal of Geophysical Research: Atmospheres, 120(3):1186-1201.

van Heerwaarden, C. C., Mellado, J. P., and De Lozar, A. (2014). Scaling laws for the heterogeneously heated free convective boundary layer. Journal of the Atmospheric Sciences, 71(11):3975-4000.

van Heerwaarden, C. C., Vilà-Guerau de Arellano, J., Moene, A. F., and Holtslag, A. A. M. (2009). Interactions between dry-air entrainment, surface evaporation and convective boundary-layer development. Quarterly Journal of the Royal Meteorological Society, 135(642):1277-1291.

van Heerwaarden, C. C., Vilà-Guerau de Arellano, J., Gounou, A., Guichard, F., and Couvreux, F. (2010). Understanding the daily cycle of evapotranspiration: A method to quantify the influence of forcings and feedbacks. Journal of Hydrometeorology, 11(6):1405-1422.

van Kesteren, B., Hartogensis, O., van Dinther, D., Moene, A., Bruin, H. D., and Holtslag, A. (2013). Measuring h2o and co2 fluxes at field scales with scintillometry: Part ii - validation and application of 1-min flux estimates. Agricultural and Forest Meteorology, 178-179:88 - 105. Special Issue:Drought Inner Asia.

van Stratum, B. J. H., Vilà-Guerau de Arellano, J., van Heerwaarden, C. C., and Ouwersloot, H. G. (2014). Subcloud-layer feedbacks driven by the mass flux of shallow cumulus convection over land. Journal of the Atmospheric Sciences, 71(3):881-895. 
van Zanten, M. C., Stevens, B., Nuijens, L., Siebesma, A. P., Ackerman, A. S., Burnet, F., Cheng, A., Couvreux, F., Jiang, H., Khairoutdinov, M., Kogan, Y., Lewellen, D. C., Mechem, D., Nakamura, K., Noda, A., Shipway, B. J., Slawinska, J., Wang, S., and Wyszogrodzki, A. (2011). Controls on precipitation and cloudiness in simulations of trade-wind cumulus as observed during RICO. Journal of Advances in Modeling Earth Systems, 3(2).

Veerman, M. (2019). 3d radiative transfer effects on dynamic heterogeneities due to shading by shallow cumulus clouds over a vegetated surface. In preparation.

Vilà-Guerau de Arellano, J., Ouwersloot, H. G., Baldocchi, D., and Jacobs, C. M. J. (2014). Shallow cumulus rooted in photosynthesis. Geophysical Research Letters, 41(5):1796-1802.

Vilà-Guerau de Arellano, J., van Heerwaarden, C. C., and Lelieveld, J. (2012). Modelled suppression of boundary-layer clouds by plants in a $\mathrm{CO}_{2}$-rich atmosphere. Nature Geoscience, 5:701-704.

Vilà-Guerau de Arellano, J., van Heerwaarden, C. C., van Stratum, B. J., and van den Dries, K. (2015). Atmospheric Boundary Layer: Integrating Air Chemistry and Land Interactions. Cambridge University Press.

Vilà-Guerau de Arellano, J., Wang, X., Pedruzo-Bagazgoitia, X., Sikma, M., AgustiPanareda, A., Boussetta, S., Balsamo, G., Machado, L., Gentine, P., Martin, S., Fuentes, J., and Gerken, T. (2019). Interactions between the amazonian rainforest and cumuli clouds: A large-eddy simulation, high-resolution ecmwf and observational intercomparison study. Journal of Advances in Modeling Earth Systems. Under review.

Vogelmann, A. M., McFarquhar, G. M., Ogren, J. A., Turner, D. D., Comstock, J. M., Feingold, G., Long, C. N., Jonsson, H. H., Bucholtz, A., Collins, D. R., Diskin, G. S., Gerber, H., Lawson, R. P., Woods, R. K., Andrews, E., Yang, H.-J., Chiu, J. C., Hartsock, D., Hubbe, J. M., Lo, C., Marshak, A., Monroe, J. W., McFarlane, S. A., Schmid, B., Tomlinson, J. M., and Toto, T. (2012). RACORO extended-term aircraft observations of boundary layer clouds. Bulletin of the American Meteorological Society, 93(6):861-878.

Wang, S., Golaz, J.-C., and Wang, Q. (2008). Effect of intense wind shear across the inversion on stratocumulus clouds. Geophysical Research Letters, 35(15).

Wang, S., Zheng, X., and Jiang, Q. (2012). Strongly sheared stratocumulus convection: an observationally based large-eddy simulation study. Atmospheric Chemistry and Physics, 12(11):5223-5235.

Wood, R. (2012). Stratocumulus clouds. Monthly Weather Review, 140(8):2373-2423.

Wright, J. S., Fu, R., Worden, J. R., Chakraborty, S., Clinton, N. E., Risi, C., Sun, Y., and Yin, L. (2017). Rainforest-initiated wet season onset over the southern amazon. 
Proceedings of the National Academy of Sciences, 114(32):8481-8486.

Wyngaard, J. C. (2004). Toward numerical modeling in the "Terra Incognita". Journal of the Atmospheric Sciences, 61(14):1816-1826.

Xu, K.-M. and Randall, D. A. (1996). A semiempirical cloudiness parameterization for use in climate models. Journal of the Atmospheric Sciences, 53(21):3084-3102.

Yamaguchi, T. and Randall, D. A. (2008). Large-eddy simulation of evaporatively driven entrainment in cloud-topped mixed layers. Journal of the Atmospheric Sciences, 65(5):1481-1504.

Yu, H., Liu, S. C., and Dickinson, R. E. (2002). Radiative effects of aerosols on the evolution of the atmospheric boundary layer. Journal of Geophysical Research: Atmospheres, 107(D12):AAC 3-1-AAC 3-14.

Zhang, Y. and Klein, S. A. (2010). Mechanisms affecting the transition from shallow to deep convection over land: Inferences from observations of the diurnal cycle collected at the arm southern great plains site. Journal of the Atmospheric Sciences, 67(9):2943-2959.

Zhang, Y. and Klein, S. A. (2013). Factors controlling the vertical extent of fair-weather shallow cumulus clouds over land: Investigation of diurnal-cycle observations collected at the arm southern great plains site. Journal of the Atmospheric Sciences, $70(4): 1297-1315$.

Zhao, M. and Austin, P. H. (2005). Life cycle of numerically simulated shallow cumulus clouds. part i: Transport. Journal of the Atmospheric Sciences, 62(5):1269-1290.

Zhuang, Y., Fu, R., Marengo, J. A., and Wang, H. (2017). Seasonal variation of shallowto-deep convection transition and its link to the environmental conditions over the central amazon. Journal of Geophysical Research: Atmospheres, 122(5):2649-2666.

Zouzoua, M. (2019). Nocturnal low-level stratiform clouds breakup during southern west african monsoon season. In preparation. 


\section{Acknowledgements}

In the way in which the credits and mentions roll at the end of a movie, I would like to do the same here. However, given that a movie usually lasts about 90 minutes and this book took 4 years, I am sure the reader will allow me a bit more freedom in extending my gratitude.

Firstly I would like to thank my research colleague, trip mate, dinner partner and fellow in rainforest adventures, who happens to be the same person as my daily supervisor and promotor. Thank you immensely, Jordi, for giving me the chance to discover what science is, how it is done, how the life of a scientist is and what it takes to become one. I appreciate your willingness to listen, critical attitude, curiosity and, above all, the fact that you were always there when needed for a discussion, an answer or another question. I felt I had at the reach of my hand someone with the knowledge of almost 40 years of science and the willingness to discuss of a PhD student. I am also thankful to my football coaches in the past that made it easier for me to understand your metaphors.

Of course the content of this book cannot be attributed just to one or two people. I would like to thank both Eduardo and Cor Jacobs for your time even before I started the thesis, to Chiel for your short but deep tips, opinions and comments, and to Huug for your admirable sharpness in anything you do, including giving me feedback. Thank you Martin for your work, time and comments especially in the first years, and for the dinners and talks in the last ones. Also, I want to thank you Menno for your contribution and work in my final stages. I would also like to show my gratitude to Fabian, Jimy and Pedro for your help during the respective work I did with each of you along my thesis.

In the process of learning what research is and how to keep a scientific discussion, communities like the one created around the BBOS-BLT meetings are definitely necessary and useful. I would like to thank each and all of the speakers and attendants of this informal but no-nonsense meetings. I would particularly like to thank you, Stephan, for your interest and eagerness to discuss as long and as deep as needed both about work you were involved in and about other in the same way. I acknowledge as well your precise and honest feedback when helping me.

I would not like to continue without saying thanks to you, Fabienne, for the opportunity you gave me to see, touch, and taste what an international research project, as well as a true scientific experimental campaign, are. I learned a lot about science and everything it involves thanks to it, and I truly appreciate your work and effort in making me feel part of a team. Bianca, Cheikh, Karmen, Marie, Norbert: thank you for your time, tips and efforts in understanding my idealized world. I would also like to thank everyone who contributed in one way or another to the DACCIWA project and the field campaign.

A supportive research group where the scientific debate is as present as a good atmosphere 
is tremendously helpful in pursuing a $\mathrm{PhD}$. Thanks to all of you at MAQ for making my $\mathrm{PhD}$ time quite enjoyable. Of course, only $\mathrm{PhD}$ fighters can sometimes understand script-derived frustration or beer-induced stupidities. Thank you all, and specially Alba for your Sun, Anja for your sense of humour, Aris for your eloquence, Auke for your FRY professorship, Imme for your mood and singing, Marie for your pillow and Stijn for your acute rhyming skills and tolerance to complains. Related to this, thank you to the university campus for not distracting me from my purpose here with unnecessary tasty meals. Although already a PhD, it was fun and a socio-cultural adventure to meet you, Wei.

Teaching and supervising is also the best way to learn. Thank you Sjoerd, Xuemei, and Felipe for your energy and willingness to learn, and thank you to all the "AVSI" and "Boundary-Layer Processes" students who had to stand me and my explanations. At least I enjoyed it a lot, and I hope you learnt something out of it.

Thanks to my dear community where I was lucky to live, because 6 years there are somehow an accelerated version of life itself. You arrive full of excitement, open to everything, willing to invest time in meeting people, in joining activities, in learning, in doing as much as you can although you are not really sure of what is the most adequate or most necessary. You then slowly start to understand how things work, what people you rather spend more time with, and start to see how you can have a meaningful contribution. And by the end, you have less people around, but in a honest relationship also allowing for not so full-of-energy moments but equally necessary. In the meantime you meet fantastic people, that fill your soul with joy, your body with energy and show you that in our world happiness is very often a choice and not a consequence of your situation. Among this people, I find many of my most dear friends in the last years: Berta, Gerardo, Maria, Carmela, Carito, Shan, Ramon, Mara, Mariana, Maaike, Agata, Serena, Jordi and many others, you know who you are. All people who arrive, shine at its fullest and eventually, leave. The difference between living in this community and real life is that, when people leave, you do not put flowers on a graveyard and cry for them. Instead, you have a net full of colourful dots around the world that lightens your eyes every time you think of each other, and that are and will always be waiting for you to stop by for a few seconds, hours or days, and remind you again: that happiness is very often a choice not only for the time spent here, but for the rest of your life. My dear friends, thank you. And specially extra thanks to my paranymphs for their contribution to a smooth organization and defence (that's at least what I hope at the time of writing this).

I was similarly lucky to leave in a house full of individuals who took enough time for their housemates, their adventures, projects and defeats. I am happy I had the chance to share my space and time and to feel home around all of you: Heike, Joao, Stefano, Renato, Arta, Kohji, Matias, Florie, Ine, David, Anais, Emilio, Maura, Marco, Yannis, Matika and others. Sorry for the dirty dishes (if ever happened), thank you for the fun, 
and hope to see you all again. Let me know if you ever need a room. And of course, thanks to Arwin El Presidente for being the only one staying there from the first to the last day.

I would like to thank the contribution to this work many times forgotten by all tax-payers, both in Spain and in The Netherlands. It is the taxes payed by every citizen what allows science and society to advance. It is thanks to these taxes that the topic of this thesis was conceived, the project was started and I was hired to carry out the actual research. And it was the tax-funded state who allowed me to reach this place and position through the taxfunded public school, public high school and public university in Spain. Similarly, I would also like to thank everyone who contributed in one way or another to what Python is, and all its packages, as well as to every person that has ever contributed to the place where almost every question has at least one answer: Stack Overflow.

También quiero, cómo no, dar las gracias a mi familia. Gracias Ange y Amparo por todo, este libro tiene la culpa de que no llegara a tiempo para veros. Gracias Boni por aguantarme cuando estaba y por no olvidarme cuando no. Gracias Aita y Ama por todo lo que me habéis cuidado, ayudado, enseñado y animado ininterrumpidamente desde que recuerdo, y antes de eso también. En realidad esta tesis empezó hace unos 30 años en Pamplona y después de interrupciones, idas y venidas, termina hoy. Porque este trabajo también es tuyo, Ama.

Eta azkenik, hitz batzuk tesia egiteko bezainbeste esfortzu egin behar izan duenarentzat. Eskerrik asko zure pazientziagatik, behar nuenean ni entzuteagatik, eta behar zenean haserretzeagatik ere. Mila esker zure esfortzuagatik eta tren, kotxe eta hegazkinetan pasatu dituzun orduengatik. Edozein egoeratan, hodei ilun edo ekaitz beldurgarri guztien gainetik, beti izan zarelako eguzki distiratsu. 



\section{About the author}

Xabier Pedruzo Bagazgoitia was born in the first hours of the 21st August 1990 in Vitoria-Gasteiz with some contributions by his parents. He attended his first education years in José Mardones and López de Guereñu Public Schools, and he carried out his high school years at IES Ekialdea BHI. After the obligatory studies he started in 2008 the Grado (4-year Bachelor) in Physics in the Public University of the Basque Country (UPV-EHU) following the "Funda-

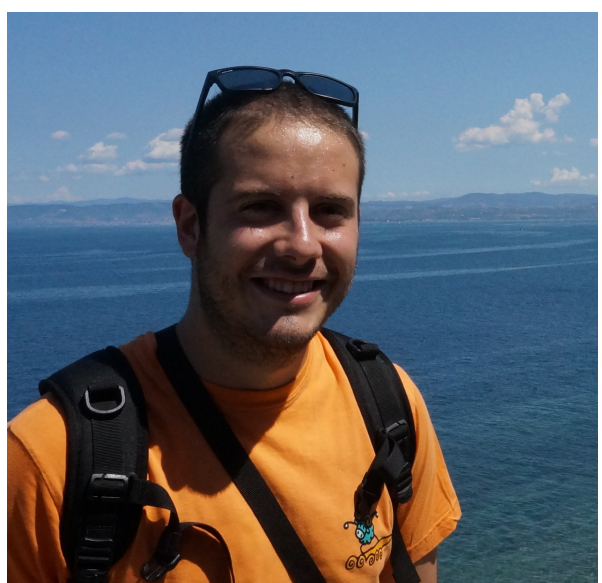
mental Physics" specialization. He spent the academic year 2011-2012 at Leiden University in The Netherlands thanks to the Erasmus Programme. That stay included the realization of a Bachelor thesis entitled "Massive neutrino models and physics beyond the Standard Model".

After finishing his Grado studies Xabier obtained a grant to follow the post-graduate course "Energy and Competitiveness. Gas, networks and renewables" by the Basque Institute of Competitiveness Orkestra in Deusto University. Following the finalization of these studies he continued in the field of sustainability and was granted a scholarship by the European Union and the Basque Government. The grant partially funded a 4 month internship at the Research Institute for Managing Sustainability at the Vienna University of Economics and Business (WU).

To learn more on the physics of the atmosphere, Xabier decided to start in 2013 the MSc programme "Climate Studies" at Wageningen University following the "Meteorology" specialization on which he graduated with Cum laude. He presented the results of his MSc Thesis at the conference "ESS 2015: A conference on Earth System Science" organized at the Max Planck Institute for Chemistry in Mainz, Germany. The curiosity to learn about the atmosphere was not satisfied and, thus he decided to start a $\mathrm{PhD}$ on the cloudvegetation interactions within the boundary layer at Wageningen University.

Besides the academic interest in physics and boundary-layer meteorology Xabier has shown interest in the field of cooperation and along his education he followed courses such as "Strategies for Cooperation to Development" organized by Setem-Hego Haizea and "Introduction to Cooperation and Technologies for Human Development" by Engineers without Borders. 


\section{Peer-reviewed journal publications}

Pedruzo-Bagazgoitia, X., Ouwersloot, H. G., Sikma, M., van Heerwaarden, C. C., Jacobs, C. M. J., and Vilà-Guerau de Arellano, J. (2017). Direct and diffuse radiation in the shallow cumulus-vegetation system: Enhanced and decreased evapotranspiration regimes. Journal of Hydrometeorology, 18(6):1731-1748

Pedruzo-Bagazgoitia, X., Jiménez, P. A., Dudhia, J., and Vilà-Guerau de Arellano, J. (2019a). Shallow cumulus representation and its interaction with radiation and surface at the convection gray zone. Monthly Weather Review, 147(7):2467-2483

Pedruzo-Bagazgoitia, X., de Roode, S. R., Adler, B., Babić, K., Dione, C., Kalthoff, N., Lohou, F., Lothon, M., and Vilà-Guerau de Arellano, J. (2019b). The diurnal stratocumulus-to-cumulus transition over land. Atmospheric Chemistry and Physics Discussions, 2019:1-30

Pedruzo-Bagazgoitia, X., Veerman, M., Jakub, F., van Heerwaarden, C. C., and VilàGuerau de Arellano, J. (In preparation). Emergent features on cloud convection by coupling $3 \mathrm{~d}$ radiation and surface on large eddy simulation

Kalthoff, N., Lohou, F., Brooks, B., Jegede, G., Adler, B., Babić, K., Dione, C., Ajao, A., Amekudzi, L. K., Aryee, J. N. A., Ayoola, M., Bessardon, G., Danuor, S. K., Handwerker, J., Kohler, M., Lothon, M., Pedruzo-Bagazgoitia, X., Smith, V., Sunmonu, L., Wieser, A., Fink, A. H., and Knippertz, P. (2018). An overview of the diurnal cycle of the atmospheric boundary layer during the west african monsoon season: results from the 2016 observational campaign. Atmospheric Chemistry and Physics, 18(4):2913-2928

Lobos-Roco, F., Vilà-Guerau de Arellano, J., and Pedruzo-Bagazgoitia, X. (2018). Characterizing the influence of the marine stratocumulus cloud on the land fog at the atacama desert. Atmospheric Research, 214:109 - 120

Sikma, M., Ouwersloot, H. G., Pedruzo-Bagazgoitia, X., van Heerwaarden, C. C., and Vilà-Guerau de Arellano, J. (2018). Interactions between vegetation, atmospheric turbulence and clouds under a wide range of background wind conditions. Agricultural and Forest Meteorology, 255:31 - 43. Honoring W.J. Massman's Discoveries: Bringing Physics to Agriculture 
Adler, B., Babić, K., Kalthoff, N., Lohou, F., Lothon, M., Dione, C., Pedruzo-Bagazgoitia, X., and Andersen, H. (2019). Nocturnal low-level clouds in the atmospheric boundary layer over southern west africa: an observation-based analysis of conditions and processes. Atmospheric Chemistry and Physics, 19(1):663-681

Babić, K., Adler, B., Kalthoff, N., Andersen, H., Dione, C., Lohou, F., Lothon, M., and Pedruzo-Bagazgoitia, X. (2019). The observed diurnal cycle of low-level stratus clouds over southern west africa: a case study. Atmospheric Chemistry and Physics, 19(2):12811299

Dione, C., Lohou, F., Lothon, M., Adler, B., Babić, K., Kalthoff, N., Pedruzo-Bagazgoitia, X., Bezombes, Y., and Gabella, O. (2019). Low-level stratiform clouds and dynamical features observed within the southern west african monsoon. Atmospheric Chemistry and Physics, 19(13):8979-8997

Lohou, F., Kalthoff, N., Adler, B., Babić, K., Dione, C., Lothon, M., Pedruzo-Bagazgoitia, X., and Zouzoua, M. (2019). Conceptual model of diurnal cycle of stratiform low-level clouds over southern west africa. Atmospheric Chemistry and Physics Discussions, 2019:125

Sikma, M., Vilà-Guerau de Arellano, J., Pedruzo-Bagazgoitia, X., Voskamp, T., Heusinkveld, B., Anten, N., and Evers, J. (In press). Impact of future warming and enhanced [co2] on the vegetation-cloud interaction. Journal of Geophysical Research: Atmospheres, n/a(n/a)

Vilà-Guerau de Arellano, J., Wang, X., Pedruzo-Bagazgoitia, X., Sikma, M., AgustiPanareda, A., Boussetta, S., Balsamo, G., Machado, L., Gentine, P., Martin, S., Fuentes, J., and Gerken, T. (Under review). Interactions between the amazonian rainforest and cumuli clouds: A large-eddy simulation, high-resolution ecmwf and observational intercomparison study. Journal of Advances in Modeling Earth Systems 


\title{
SENSE
}

Netherlands Research School for the

Socio-Economic and Natural Sciences of the Environment

\section{I P L O M A}

for specialised PhD training

The Netherlands research school for the

Socio-Economic and Natural Sciences of the Environment

(SENSE) declares that

\section{Xabier Pedruzo Bagazgoitia}

\author{
born on 21 August 1990 in Vitoria-Gasteiz, Spain
}

has successfully fulfilled all requirements of the educational PhD programme of SENSE.

Wageningen, 6 December 2019

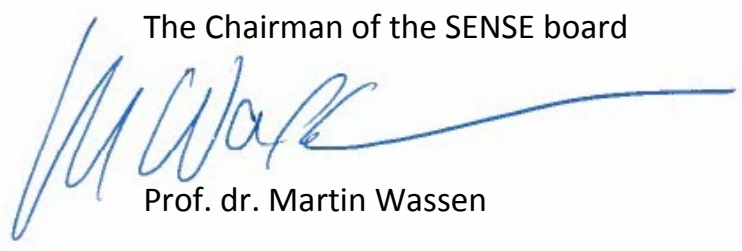

the SENSE Director of Education

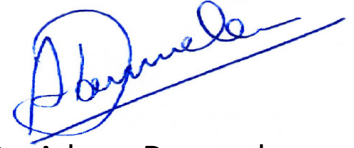

Dr. Ad van Dommelen

The SENSE Research School has been accredited by the Royal Netherlands Academy of Arts and Sciences (KNAW)

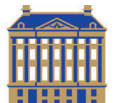

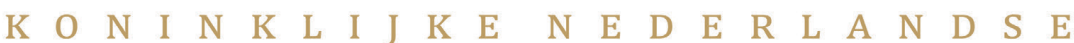

$\begin{array}{lllllllllllllllllllllllll}\text { A } & K & \text { A } & \text { D } & \text { E } & \text { M } & \text { I } & \text { E } & \text { V } & \text { A } & \text { N } & \text { W } & \text { E } & \text { T } & \text { E } & \text { N } & \text { S } & \text { C } & \text { H } & \text { A } & \text { P } & \text { P } & \text { E } & \text { N }\end{array}$ 


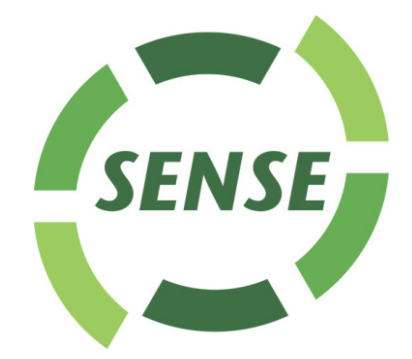

The SENSE Research School declares that Xabier Pedruzo Bagazgoitia has successfully fulfilled all requirements of the educational PhD programme of SENSE with a work load of $42.4 \mathrm{EC}$, including the following activities:

\section{$\underline{\text { SENSE PhD Courses }}$}

o Environmental research in context (2016)

- Research in context activity: 'Organization of "A workshop on Boundary -Layer meteorology: Understanding the present and thinking the future", Barcelona, 14 October 2016

\section{Other PhD and Advanced MSc Courses}

- Workshop 'Atmospheric observational techniques', University Paul Sabatier, France (2016)

- Career Perspectives, Wageningen Graduate Schools (2019)

\section{Selection of External training at a foreign research institute}

- Participant in DACCIWA observational campaign, University Paul Sabatier, Benin (2016)

- Guest researcher at the National Center for Atmospheric Research, USA (2017)

\section{Management and Didactic Skills Training}

- Co-supervising two MSc student internships (2018)

- Co-supervising BSc student with thesis entitled 'Sun and plants: The challenging link and its impact on the Atmospheric Boundary Layer' (2016)

- Assistant teacher in the BSc courses 'Clouds in present and changing climate' (20162017), 'Atmosphere-Vegetation-Soil Interactions' (2016-2017) and 'Boundary Layer Processes' (2017-2018)

- Co-organisation of workshop on land-atmosphere interactions and the CLASS software principles at EGU 2019, Vienna, Austria

- Co-organisation of workshop: "Large Eddy Simulations and mixed-layer models as tools to investigate land-atmosphere interactions", National Institute for Space Research Center for Weather Forecast and Climate Studies, Brazil (2019)

\section{Selection of Oral Presentations}

- The Dynamical and Radiative Effects of Shallow Cumulus below, in and Above the Gray Zone. 23rd Symposium on Boundary Layers and Turbulence, American Meteorological Society, 11-15 June 2018, Oklahoma City, United States of America

- The stratocumulus to cumulus transition: Quantifying a typical southern West Africa case through LES. European Geosciences Union Annual Assembly, 7-12 April 2019, Vienna, Austria

SENSE coordinator PhD education

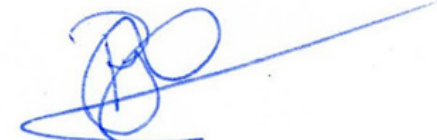

Dr. ir. Peter Vermeulen 
This research received funding from the Nederlandse Organisatie voor Wetenschappelijk Onderzoek (NWO) under the project name "A novel approach to understand and regionally represent vegetation-cloud interactions".

Computer simulations were financially supported by the Netherlands Organisation for Scientific Research (NWO) Physical Science Division under the project 16666. We acknowledge SurfSARA for the technical support.

Funding from the DACCIWA project is thanked for the realization of Chapter 4.

Financial support from Wageningen University for printing this thesis is gratefully acknowledged.

Cover design by Adrian Rivera Tchernikov with author's contribution. 
